\title{
Strategies for chronic inguinal pain
}

Citation for published version (APA):

Zwaans, W. A. R. (2017). Strategies for chronic inguinal pain. [Doctoral Thesis, Maastricht University]. Maastricht University. https://doi.org/10.26481/dis.20171117wz

Document status and date:

Published: 01/01/2017

DOI:

$10.26481 / \mathrm{dis} .20171117 \mathrm{wz}$

Document Version:

Publisher's PDF, also known as Version of record

\section{Please check the document version of this publication:}

- A submitted manuscript is the version of the article upon submission and before peer-review. There can be important differences between the submitted version and the official published version of record.

People interested in the research are advised to contact the author for the final version of the publication, or visit the DOI to the publisher's website.

- The final author version and the galley proof are versions of the publication after peer review.

- The final published version features the final layout of the paper including the volume, issue and page numbers.

Link to publication

\footnotetext{
General rights rights.

- You may freely distribute the URL identifying the publication in the public portal. please follow below link for the End User Agreement:

www.umlib.nl/taverne-license

Take down policy

If you believe that this document breaches copyright please contact us at:

repository@maastrichtuniversity.nl

providing details and we will investigate your claim.
}

Copyright and moral rights for the publications made accessible in the public portal are retained by the authors and/or other copyright owners and it is a condition of accessing publications that users recognise and abide by the legal requirements associated with these

- Users may download and print one copy of any publication from the public portal for the purpose of private study or research.

- You may not further distribute the material or use it for any profit-making activity or commercial gain

If the publication is distributed under the terms of Article $25 \mathrm{fa}$ of the Dutch Copyright Act, indicated by the "Taverne" license above, 
STRATEGIES

FOR CHRONIC

INGUINAL PAIN

Willem A.R. Zwaans 


\section{STRATEGIES FOR CHRONIC INGUINAL PAIN}

WILLEM A.R. ZWAANS 
Publication of this thesis was financially supported by:

Máxima Medisch Centrum - SolviMáx expertise centrum voor buikwand- en liespijnMMC Academie - Maastricht Universitair Medisch Centrum - Maastricht UniversityResearch Manager - CoMedical - Schwa Medico

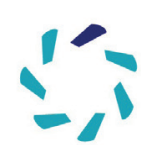

máxima medisch centrum
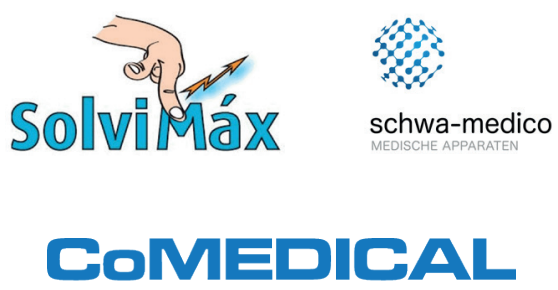

Radiofrequency Pain Management
Maastricht UMC+

\section{Colofon}

Author:

Willem A.R. Zwaans

Cover design and lay-out:

Miranda Dood, Mirakels Ontwerp

Printing:

Gildeprint - The Netherlands

ISBN:

(C) Willem A.R. Zwaans, Eindhoven, The Netherlands, 2017.

All rights reserved. No part of this publication may be reproduced or transmitted in any form by any means, without permission of the author. 


\section{STRATEGIES FOR CHRONIC \\ INGUINAL PAIN}

\section{PROEFSCHRIFT}

ter verkrijging van de graad van doctor aan de Universiteit Maastricht, op gezag van de Rector Magnificus, Prof. dr. Rianne M. Letschert volgens het besluit van het College van Decanen, in het openbaar te verdedigen op vrijdag 17 november 2017 om 14.00 uur

door

Willem Antonius Richardus Zwaans geboren op 25 februari 1988 te Schaijk 


\section{Promotor}

Prof. dr. M. van Kleef

\section{Copromotores}

Dr. R.M.H. Roumen, Máxima Medisch Centrum Eindhoven/Veldhoven

Dr. M.R.M. Scheltinga, Máxima Medisch Centrum Eindhoven/Veldhoven

\section{Beoordelingsscommissie}

Prof. dr. N.D. Bouvy (voorzitter)

Prof. dr. F.J. Huygen (Erasmus Medisch Centrum Rotterdam)

Dr. S.W. Nienhuijs (Catharina Ziekenhuis Eindhoven)

Dr. M. Sommer

Prof. dr. L.P. Stassen 
Voor Leonie 


\section{TABLE OF CONTENTS}

Chapter 1 Introduction and outline

Partially published in 'The Art of Hernia - A Step by Step Guide', Chapter 41 (Aetiology, Pathology and Assessment of Chronic Pain after Inguinal Hernia Repair), edited by G. Campanelli

Chapter 2 Randomized clinical trial comparing self-gripping mesh with a standard polypropylene mesh for open inguinal hernia repair. Br J Surg 2016; 103: 812-818

Chapter 3 Groin pain characteristics and recurrence rates: Long-term results of a randomized controlled trial comparing self-gripping Progrip ${ }^{\mathrm{max}}$ mesh and sutured polypropylene mesh for open inguinal hernia repair. Ann Surg 2017 [Epub ahead of print]

Chapter 4 Mesh removal and selective neurectomy for persistent groin pain following Lichtenstein repair.

World J Surg 2017; 41(3): 701-712

Chapter 5 Laparoscopic mesh removal for otherwise intractable inguinal pain following endoscopic hernia repair is feasible, safe and may be effective in selected patients.

Surg Endosc 2017 [Epub ahead of print]

Case I A remarkable pitfall in inguinal open mesh herniorrhaphy.

Gastrointest Dig Syst 2015; 5: 5

Chapter 6 Factors determining outcome after surgery for chronic groin pain following a Lichtenstein hernia repair.

World J Surg 2015; 39: 2652-2662

6 | Strategies for chronic inguinal pain 
Chapter 7 Chronic postoperative groin pain requiring remedial surgery: Spinal or general anaesthesia?

Under review

Case II Symptomatic attachment of appendix vermiformis following a plug inguinal hernia repair.

Under review

Chapter 8 Spinal versus general anaesthesia in surgery for inguinodynia (SPINASIA trial): study protocol for a randomized controlled trial.

Trials 2017; 18: 23

Chapter 9 Recall bias in pain scores evaluating abdominal wall and groin pain 174 surgery.

Under review

Chapter 10 Summary, conclusions and general discussion

Chapter 11 Future perspectives

Chapter 12 Valorisation

List of publications

Curriculum vitae auctoris 



\section{CHAPTER 1}

\section{Introduction and outline}

This chapter was partially published in 'The Art of Hernia - A Step by Step Guide', Chapter 41 (Aetiology, Pathology and Assessment of Chronic Pain after Inguinal Hernia Repair), edited by G. Campanelli 


\section{INTRODUCTION}

\section{History of inguinal hernia repair}

The name hernia is derived from the Greek word hernios meaning bud or offshoot ${ }^{1}$. The first descriptions of hernias were found in manuscripts of Mesopotamia and ancient Egypt $^{2}$. The evolution of inguinal hernia repair can be divided into five eras as illustrated in a time line (figure 1.1). During the oldest era lasting into the fifteenth century, treatment was non-invasive. The Egyptians employed bandages that were replaced by trusses as initiated by Guy de Chauliac during the middle ages.

The start of true herniology (the second era) was characterized by barber-surgeons performing the first invasive procedures for inguinal hernias. The Frenchman Ambroise Paré castrated patients with a hernia and this approach became common practice during the Renaissance ${ }^{2}$. In addition to the castration, wound cauterization or hernia sac debridement were performed. Little attention was paid to the groin anatomy until the seventeenth century. During this (third) 'anatomic era', autopsies and anatomic dissections became fashionable throughout Europe leading to a better anatomic knowledge of the inguinal canal ${ }^{1}$. By the end of the nineteenth century, new hernia repair techniques including ligation of the hernia sac were introduced. The Prussian surgeon Billroth described excision and ligation of the peritoneal sac through the superficial inguinal ring in $1877^{3}$. MacEwen plicated the hernial sac into a plug that subsequently blocked the internal ring ${ }^{4}$. One year later, in 1887, Bassini made a paramount step in inguinal hernia repair by adopting the MacEwen procedure using reinforcement of the abdominal wall by a suture lined tension repair ${ }^{5}$. By using this method, the need for (postoperative) trusses was obviated.

Bassini's technique revolutionized inguinal hernia repair and formed the basis for many, if not all, contemporary 'tension' repairs. After the introduction of Bassini repair, the fourth epoch of hernia repair under tension started in the nineteenth century. After World War II, the Canadian Shouldice presented a tension repair that is still considered gold standard nowadays. He adopted Bassini's repair by using a four-layered tissue reconstruction technique ${ }^{6}$. In 1966, McVay added a relaxing muscle fascia incision to reduce tension in these 'tension' repairs?. This incision is considered one of the last milestones in tension repairs as an alternative technique was by then popularized, a so called tension-free repair. 


\section{Mesh introduction for inguinal hernia repair}

During the third era of inguinal hernia repair, the first descriptions of implantation of foreign material emerged. Silver was used for its bacteriocidal properties whereas plastic was introduced to strengthen the posterior wall of the inguinal canal ${ }^{8}$. However, a suture tension repair remained the standard until 1958. The American surgeon Usher published on his technique using a polypropylene mesh ${ }^{9}$. Stoppa proposed in an article 'La grande prosthese de reinforcement du sac visceral' placement of a large mesh in the preperitoneal space that was thought to support fascial defects ${ }^{10}$. The Stoppa approach may be considered as the grandfather of current laparo-endoscopic repair techniques.

The use of the body's own material may result in a significantly increased tension on tissues. In contrast, the introduction of mesh allows for performing of a tension-free repair. These tension-free techniques characterize the fifth epoch of hernia repair that started in the mid-twentieth century. In 1986, the Lichtenstein repair described by the eponymous surgeon made an entrance ${ }^{11}$. The principle of this repair is a polypropylene mesh overlapping the inguinal defect to reinforce the abdominal wall. This effective technique further popularized mesh-based hernia repair. Minimally invasive laparoendoscopic repair techniques in the 19-eighties ${ }^{12}$ and the recent introduction of robotics ${ }^{13}$ have hitherto not been able to replace the open use of mesh repair as the gold standard in most areas of the world. At present, an open Lichtenstein is preferred to a primary suture repair.

\section{Chronic postoperative inguinal pain (CPIP)}

As hernia recurrence rates have dropped substantially following the standard use of mesh $^{14}$, research attention has shifted towards groin pain after hernia repair. Although some suggest that postherniorrhaphy pain often diminishes over time ${ }^{15,16}$, some patients may develop a chronic inguinodynia. Chronic postoperative inguinal pain (CPIP), defined by the International Association for the Study of Pain (IASP) as 'pain beyond three months after inguinal hernia surgery'17, is currently the most disabling and costly complication of hernia surgery ${ }^{18,19}$. At present, CPIP is the most important patient related outcome measure (PROM) in hernia surgery.

Pooled incidences of CPIP following open mesh-based repairs, as in the Lichtenstein technique, vary between 11 and $17 \%^{16,20-22}$. Up to 6\% experience significant restrictions in social and daily activities leading to an impairment of health status and lower quality of life (QoL) ${ }^{23-27}$. Consequently, strategies to reduce CPIP are currently extensively studied. 
One strategy aimed at preventing CPIP is related to the surgical technique. The introduction of minimally invasive approaches $\left(\mathrm{TAPP}^{28}, \mathrm{TEP}^{29}\right.$ ) for hernia repair has resulted in lower CPIP incidences (6 to $12.4 \%^{22,27}$ ). CPIP rates after alternative techniques including single incision laparoscopic repair (SILS) ${ }^{30}$, robotic (assisted) repair ${ }^{13,31}$, preperitoneal (transrectus sheath preperitoneal mesh repair (TREPP) ${ }^{32,33}$ and transinguinal preperitoneal mesh-plasty (TIPP) ${ }^{34}$ are currently studied. Nevertheless, data on long-term pain beyond three years following these alternative techniques are exceedingly scarce. Therefore, mesh-based conventional open and endoscopic techniques are still recommended by international guidelines ${ }^{35}$.

A second approach to minimize CPIP focuses on the quest of the ideal type of mesh. Apart from mesh weight, elasticity, strength and porosity, the use of stitches, as advised in a conventional Lichtenstein repair, possibly increases the risk of CPIP ${ }^{35}$. Theoretically, each foreign material has a potential of inducing an inflammatory reaction and mechanical pressure.

\section{CPIP as complication of other surgery}

Nowadays, chronic inguinodynia has gained a lot of attention in inguinal hernia surgery and is sometimes only considered as complication of inguinal hernia surgery. The majority of chronic inguinodynia is indeed CPIP although chronic pain may also be related to groin conditions other than the original hernia. These conditions have received little attention and include pain following a caesarean incision (post-Pfannenstiel pain syndrome $)^{36,37}$, posttraumatic groin pain, endometriosis of the uterus' round ligament ${ }^{38}$ or inguinodynia following urologic operations ${ }^{39}$. Underlying pathophysiological mechanisms largely overlap with those of CPIP, but can also include indirect traumatic neuroma formation, migration of endometrial tissue or idiopathic diseases of the nervous system. 


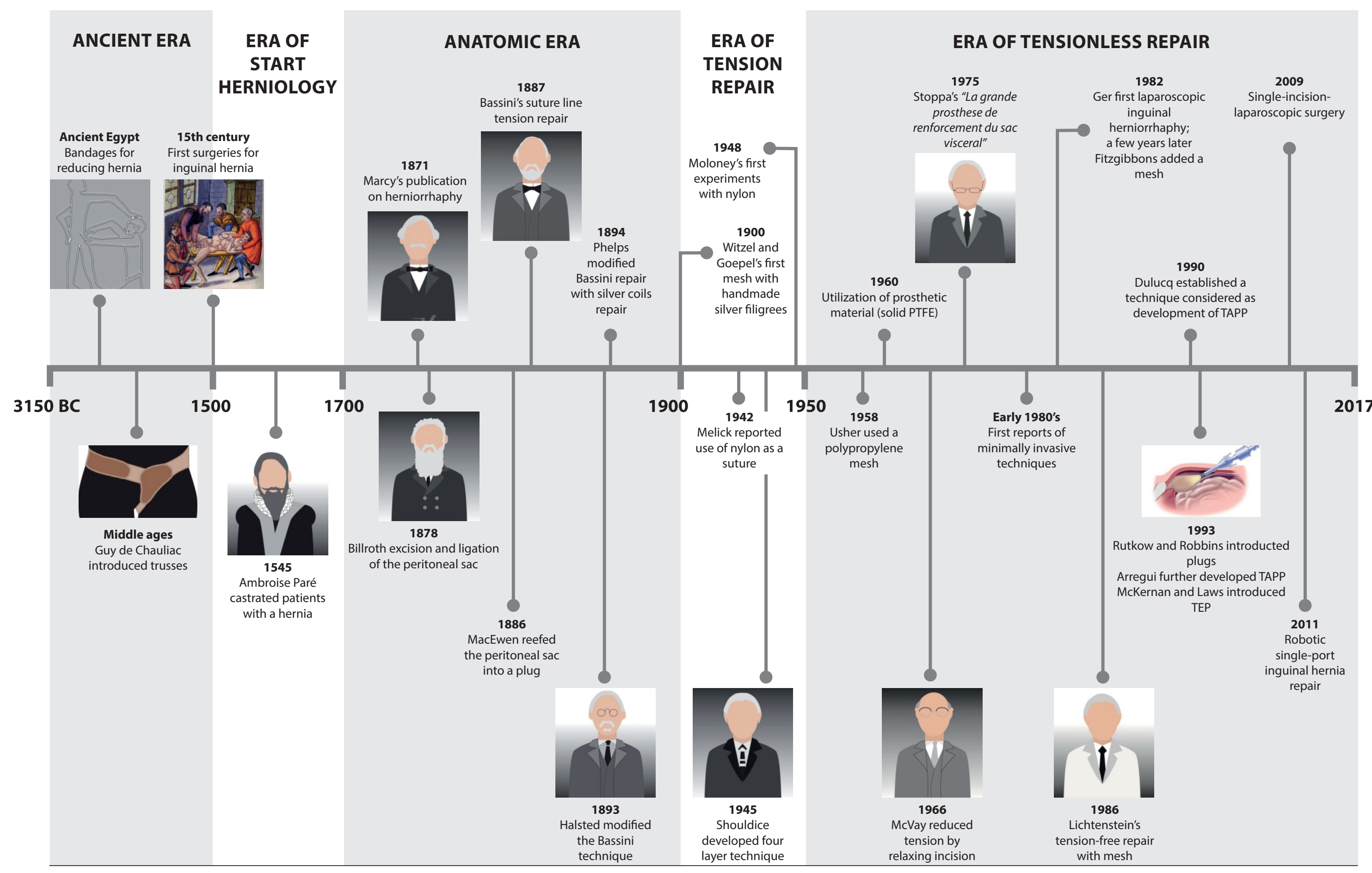

FIGURE 1.1:

Historic time line of inguinal hernia repair. 
Neuropathic pain

CPIP is generally classified as neuropathic or as non-neuropathic (inflammatory or nociceptive) pain. Neuropathic CPIP may be a result of nerve entrapment, either due to the immediate mechanical effect of the inserted mesh or following direct damage to inguinal nerves during surgery ${ }^{20,40,41}$. Incidentally, long-term neuropathic CPIP is related to traumatic neuroma formation (leading to ectopic excitability), perineural scar tissue development, entrapment of nerves due to fibrosis and peripheral or central sensitization ${ }^{42,43}$.

Central in the patient's history is a sharp, burning or shooting painful sensation which is progressive after repetitive stimulation. Paresthesia (tingling, crawling, or electrical sensations) and dysaesthesia (spontaneous or evoked unpleasant abnormal sensation) with radiation towards the associated skin area of the involved inguinal nerve are often reported. Depending on the affected nerve, pain may radiate towards the upper medial thigh (ilioinguinal nerve), suprapubic region (iliohypogastric nerve) or the genitals or ventral upper leg (genitofemoral nerve).

During physical examination, neuropathic CPIP is characterised by the presence of a point of maximum pain at palpation. This small area is covered by a somewhat larger skin region exhibiting abnormal sensation. Pain sensation following pinching may be disproportionally painful (hyperalgesic). Positive sensory abnormalities such as hyperalgesia, allodynia (pain due to a stimulus that does not normally provoke pain), or hyperpathia (abnormally painful reaction to a stimulus, especially a repetitive stimulus, as well as an increased threshold) support the diagnosis ${ }^{44}$. Moreover, negative neurophysiologic phenomena including hypoaesthesia (reduced sensation of non-painful stimuli) and hypoalgesia (diminished pain in response to a normally painful stimulus) also contribute to the picture ${ }^{44}$. Scoring a neuropathic pain diagnostic questionnaire (DN4) may be helpful in differentiating neuropathic pain from non-neuropathic pain ${ }^{45}$.

\section{Non-neuropathic pain}

Non-neuropathic or nociceptive CPIP may be a consequence of a mesh- or sutureinduced inflammatory reaction within the inguinal area that is usually ongoing for several months following inguinal hernia repair ${ }^{27}$. An example of such an inflammatory-related 
pain syndrome is periostitis of the pubic bone. Since meshes have the tendency to wrinkle and fold over time ${ }^{46}$, the formation of a so-called meshoma can also lead to pain by a volume effect or by mechanical pressure on surrounding structures. Patients with nonneuropathic CPIP report a throbbing or nagging pain located in a non-neuroanatomical area $^{47}$. Local pain increases if pressure is applied to either the mesh (mesh-related), the pubic bone (periostitis) or the funiculus (funiculodynia) ${ }^{41}$. Mesh-related CPIP is suspected when patients complain of a foreign body sensation or feeling of tightness in the groin area. The pain is often aggravated during car driving or leg crossing, whereas hip extension may relieve the pain. During physical examination, a diffuse painful palpation along the Poupart's ligament and lack of sensory loss may contribute to the diagnosis. Sometimes, the mesh can be palpated in non-obese individuals.

\section{Diagnosing CPIP}

The exact diagnosis of type of CPIP mainly depends on concise history taking and an extensive physical examination. It is imperative to appreciate that imaging techniques consistently fail to contribute to a proper diagnosis. Ultrasonography is only useful in excluding a hernia recurrence as a cause of pain. One study demonstrated that Magnetic Resonance Imaging (MRI) may confirm the presence of a flat non-wrinkled mesh, but specific causes of CPIP are not identified ${ }^{48}$. A meshoma, defined as folding and wrinkling of the inserted mesh, varying from a mass-like density to a more subtle wrinkling or fibrosis, can occasionally be seen on computed tomography (CT) or MRI, but radiologists only recognize a meshoma when they are familiar with this phenomenon ${ }^{46}$. Furthermore, its presence does not bear a relationship with the presence of CPIP. In rare cases, MRI may reveal a neuroma but the clinical value of this finding is unclear.

A diagnostic local nerve block may aid in confirming the diagnosis of neuropathic inguinodynia ${ }^{49}$, 50. However, it must be recognized that differentiation between neuropathic and non-neuropathic pain is often difficult, if not impossible, as objective diagnostic measurements are currently lacking ${ }^{51}$. In addition, a combined pain syndrome entailing neuropathic and nociceptive elements is not uncommon following hernia repair. For instance, dysejaculation ${ }^{52-54}$ (painful ejaculation) or orchialgia ${ }^{55,56}$ may be observed during both types of pain. 


\section{Therapy for CPIP}

\section{Conservative treatments}

First line management of CPIP is pharmacological using conventional analgesics. Neuropathic pain medications may be useful if neuropathic characteristics are present. Peripheral nerve blocks using local anaesthetic agents (possibly combined with corticosteroids) may be diagnostic but may also lead to pain reduction up to several weeks to months ${ }^{57}$. It is recognized that adequate pharmacological pain management is important in the acute phase as a means to minimize the risk of conversion to chronic pain $^{58,59}$. However, literature on the effect of pharmacological treatment for CPIP is scarce precluding any scientifically based conclusions.

If conservative treatments are to no avail, more invasive therapies may be considered. Transcutaneous electric nerve stimulation (TENS) or non-surgical interventional treatments including pulsed radiofrequency (PRF), nerve root blocks or dorsal root ganglion stimulation may all offer pain relief. Surgical interventions are usually considered as a last option once pain is recalcitrant.

\section{Remedial surgery}

Surgery to relieve CPIP is known as 'remedial' surgery. Remedial surgery is performed using an open ${ }^{42,53,60-65}$ or an endoscopic approach ${ }^{66-68}$. Two major types of remedial surgery are distinguished: neurectomy and mesh removal. Sort of remedial surgery mainly depends on the assumed cause of postherniorrhaphy pain as determined by history taking and physical examination. Again, the role of imaging in this individualized approach is next to nil.

Inguinal nerve resection, either as a tailored approach (depending on type of nerve(s) presumably affected), or as triple neurectomy (standard removal of all three inguinal nerves), is a frequently performed and accepted remedial surgical technique for (neuropathic) inguinodynia ${ }^{69}$. The number of neurectomies depends on the preference of the attending surgeon. It is unknown which type is superior. A triple neurectomy is sometimes challenging as identification of all nerves in scar tissue may be demanding.

A second type of remedial surgery in CPIP patients is the removal of the surgical mesh (meshectomy), either partial or complete. This surgical procedure is considered once the mesh is implicated as the potential cause of non-neuropathic CPIP. A meshectomy 
may be complex as operating in an area of fibrotic tissue may considerably increase the chances on complications. Moreover, the posterior wall or the inguinal canal may require reinforcement to avoid looming recurrent hernias. Reinforcement is performed by a synchronous or metachronous new mesh insertion ${ }^{70}$ or by a tension-repair using the body's own material ${ }^{11}$. Despite international guidelines to consider mesh removal in selected cases $^{35}$, this type of remedial surgery remains controversial.

Alternative types of remedial surgery for CPIP may entail the removal of fixating devices such as sutures or staples. A release of the funiculus (funiculolysis) may be helpful in a subgroup of patients reporting pain following ejaculation ${ }^{53}$. In highly selected cases, an orchiectomy (removal of the testicle $)^{72}$ or testicular denervation ${ }^{73,74}$ for intractable testicular pain may be considered. These procedures are infrequently required but may offer pain relief in highly selective groups of patients ${ }^{55,56}$.

\section{Neuro-anatomy of the groin}

Four inguinal nerves are identified (figure 1.2):

- The iliohypogastric nerve (Latin: nervus iliohypogastricus) has a common trunk with the ilioinguinal nerve from the anterior ramus of the L1 nerve root of the lumbar plexus (plexus lumbalis; figure 1.3). The common trunk runs ventrally from the lumbar quadrate muscle (musculus quadratus lumborum). The iliohypogastric nerve is divided from the ilioinguinal nerve at the lateral edge of the psoas muscle (musculus iliopsoas). It enters the abdominal wall above the iliac crest and lateral from the anterior superior iliac spine to provide sensation to the suprapubic region.

- After division of the common trunk, the ilioinguinal nerve (nervus ilioinguinalis) runs inferior to the iliohypogastric nerve. It crosses the lumbar quadrate muscle anteriorly and subsequently the iliac muscle (musculus iliacus) to enter the abdominal wall after piercing internal oblique (musculus obliquus internus abdominis), lateral from the anterior superior iliac spine. The ilioinguinal nerve then passes through the superficial inguinal ring (annulus inguinalis superficialis). Its supplies sensation of the upper medial thigh, root of the penis and upper one third of the scrotum in men (figure 1.2) and root of the clitoris and upper one third of the labia majora in women. 


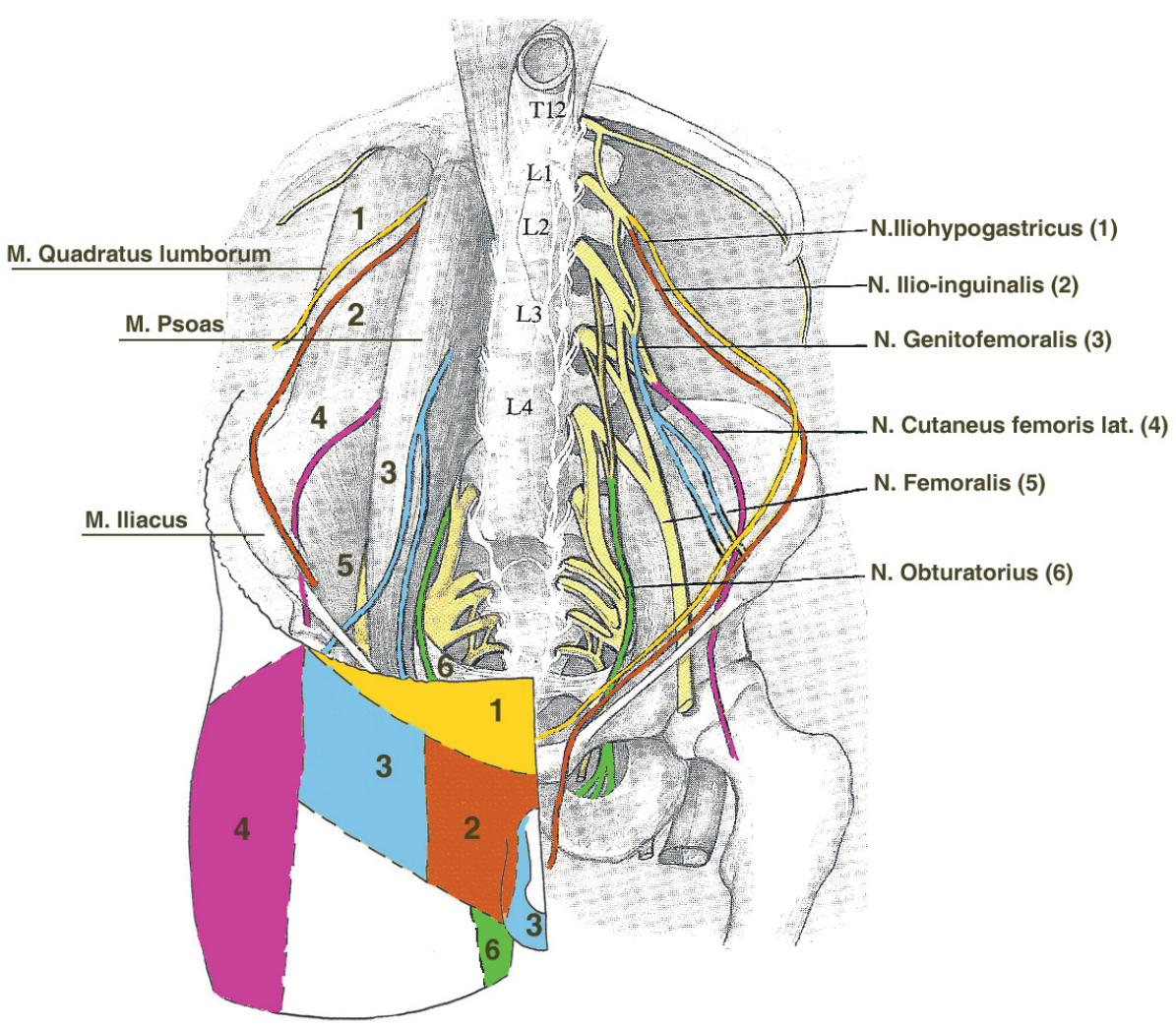

FIGURE 1.2:

Neuro-anatomy of the inguinal nerves.

- The genitofemoral nerve (nervus genitofemoralis) originates from the L1-L2 nerve root of the lumbar plexus (figure 1.3) and runs retroperitoneally on the anterior side of the (major) psoas muscle. It passes the fascia of the psoas muscle and then divides into a genital (ramus genitalis nervi genitofemoralis) and a femoral branch (ramus femoralis nervi genitofemoralis), some centimetres proximal to the inguinal ligament (ligamentum inguinale). The genital branch is more commonly injured following inguinal hernia repair, when compared to the femoral branch. The genital branch of the genitofemoral nerve enters the inguinal canal trough the deep inguinal ring (annulus inguinalis profundus) and runs along with the spermatic cord (funiculus spermaticus) in men and the round ligament of the 
uterus (ligamentum teres uteri) in women. The sensation of the scrotum and the labia majora in men and women respectively, are provided by the genital branch of the genitofemoral nerve. The femoral branch runs laterally of the external iliac artery (arteria iliaca externa) and runs inferior to the inguinal ligament. Skin sensibility of the anterior part of the upper leg is provided by the femoral branch of the genitofemoral nerve.

- The lateral femoral cutaneous nerve (nervus cutaneus femoris lateralis) is rarely affected after open inguinal hernia repair. However, laparo-endoscopic repair may result in injury to this nerve. When affected, a pain syndrome known as meralgia paraesthetica presents by a painful lateral part of the thigh. The nerve originates from the L2-L3 nerve root and emerges laterally of the psoas muscle and runs anterior of the iliac muscle. It courses caudally of the inguinal ligament and either medial or lateral to the anterior superior iliac spine ${ }^{75}$ to the sartorius muscle (musculus sartorius) where it divides into an anterior and a posterior branch.

It must be appreciated that the anatomy of the inguinal nerve can be inconsistent ${ }^{75}$. A thorough knowledge on the complex anatomy of the groin and the anatomic variations is critical in understanding the characteristics of chronic inguinodynia. Moreover, insufficient anatomical knowledge may increase the chances on CPIP after groin surgery.
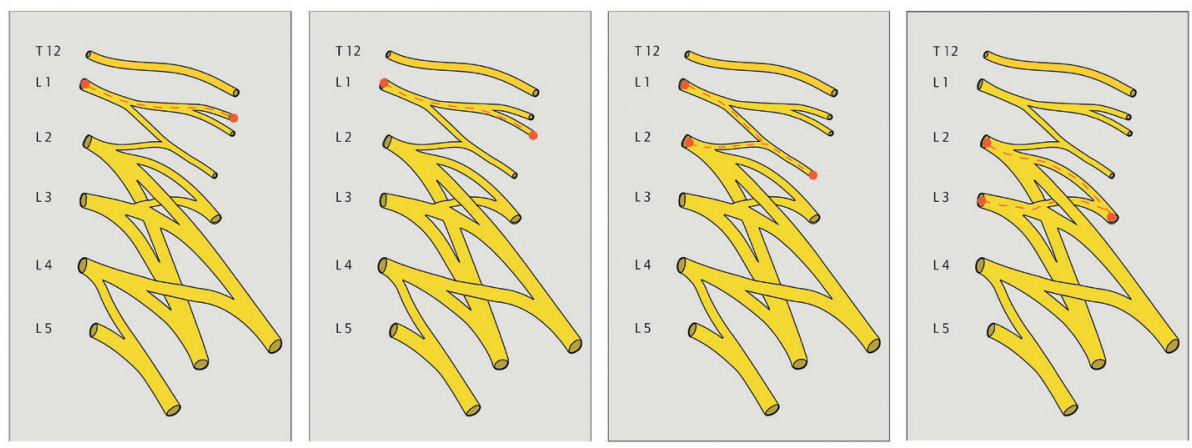

FIGURE 1.3:

Lumbar plexus with origins of the iliohypogastric, ilioinguinal and genitofemoral nerve and the lateral femoral cutaneous nerve. 


\section{Risk factors for CPIP}

Known factors to increase the onset of CPIP include a high preoperative pain intensity ${ }^{21,76,77}$, the preoperative presence of chronic pain conditions ${ }^{21}$, female gender $^{18,21,27,78}$, young age $\mathrm{e}^{21,76,77}$, general anaesthesia ${ }^{79,80}$, anterior open approach (versus laparoscopic repair) ${ }^{16,21,76}$, surgery for recurrent inguinal hernia (versus primary repair) ${ }^{23}$, incomplete identification of all three inguinal nerves ${ }^{21,81}$, use of a heavyweighted polypropylene mesh for repair ${ }^{16,21}$, severe immediate postoperative pain $^{21,40,82}$, and postoperative complications ${ }^{76,77}$. However, it must be appreciated that the relative contribution of these factors is unknown. Moreover, the role of psychological factors as expectations, anxiety, depression, past memories and social environment are recognized but their relative contribution to the development of chronic pain in general is also unclear $^{19,40,83,84}$. Risk factors for ongoing pain after the execution of remedial surgery for CPIP, however, have not been described in literature yet.

\section{Bias}

In contemporary research, self-reported pain intensity is used as an outcome measure more often than before. Given the importance of self-reported pain scores in clinical research, its reliability is critical. Retrospective studies are known for their limitations. One possible flaw of these studies is a type of information bias called recall bias. Peer reviewers are inclined to disqualify retrospective studies because the potential presence of recall bias. Currently, however, it is unclear if recall of pain scores just results in inaccurate measurements or may also lead to a significantly altered outcome of retrospective pain studies. 


\section{AIMS OF THE THESIS}

General aim of this thesis is to study prevention of CPIP and to optimize its treatment with emphasis on the role of remedial surgery. Specifics aims are the following:

\section{Prevention of CPIP}

\section{Can self-gripping meshes reduce CPIP and long-term recurrences rates?}

The results of a randomized controlled trial (RCT) comparing CPIP in patients receiving a conventional sutured polypropylene mesh according to Lichtenstein versus a lightweight self-gripping Progrip ${ }^{\mathrm{Tm}}$ mesh, omitting the use of any fixation stitches are described in chapter 2 (Randomized clinical trial comparing self-gripping mesh with a standard polypropylene mesh for open inguinal hernia repair) and chapter 3 (Groin pain characteristics and recurrence rates: Long-term results of a randomized controlled trial comparing self-gripping Progrip ${ }^{\mathrm{Tm}}$ mesh and sutured polypropylene mesh for open inguinal hernia repair).

\section{Surgical treatments for CPIP}

2. How is a mesh-related pain characterized and is an open mesh removal effective in selected patients with CPIP?

An open mesh removal may be considered in selected cases $^{35}$, but plain descriptions of clinical characteristics of a typical mesh-related pain to select these patients are currently lacking. The characteristics of this mesh-related CPIP are described to serve as a general tool of guidance for (hernia) specialists and other physicians in chapter 4: Mesh Removal and Selective Neurectomy for Persistent Groin Pain Following Lichtenstein Repair.

Mesh removal remains controversial due to the scarcity of studies and the fear of hernia recurrences and other complications. The results of a study on open mesh removal and a complementary literature study on all available evidence for mesh removal are discussed in chapter 4 . 


\section{Is laparoscopic mesh removal feasible, safe and effective in selected patients suffering from CPIP?}

An endoscopic mesh insertion for a primary inguinal hernia repair is the preferred method in Western countries nowadays. This approach is associated with accelerated recovery and an improved cost-effectiveness compared to open hernia repair ${ }^{35}$. CPIP is less prevalent following a minimally invasive procedure, but still some patients may suffer from pain. Due to increasing numbers of endoscopic mesh insertions, growing numbers of patients with CPIP following endoscopic repair are currently presenting at pain clinics. An exciting new method for the removal of these meshes is described in this thesis. Chapter 5 (Laparoscopic mesh removal for otherwise intractable inguinal pain following endoscopic hernia repair is feasible, safe and may be effective in selected patients) elaborates on anatomical issues, feasibility and safety of a laparoscopic technique for the removal of preperitoneally inserted meshes.

\section{Case: importance of following the correct sequence of surgical steps}

If the consecutive steps of a surgical technique are not followed in a correct order, pitfalls are looming. An example illustrating this contention is described in Case I: A Remarkable Pitfall in Inguinal Open Mesh Herniorrhaphy). This case of a divided funiculus following Lichtenstein hernia repair is used to make a series of recommendations for standard open repair.

\section{Optimizing surgical treatments for CPIP}

\section{How can success rates of remedial surgery for CPIP be optimized?}

Aimed at improving outcome of surgical therapies for CPIP after a conventional open mesh-based Lichtenstein technique, factors predicting treatment outcome of remedial groin pain surgery were investigated. Chapter 6: Factors Determining Outcome After Surgery for Chronic Groin Pain Following a Lichtenstein Hernia Repair is dedicated to the question if and which risk factors can be identified to predict a significant pain relief after remedial surgery for CPIP.

Interestingly, type of anaesthesia that is used during remedial surgery (spinal versus general anaesthesia) was influential in a retrospective case series of patients undergoing surgery for CPIP (Chapter 6). To test whether the results of this retrospective study are reproducible and if spinal anaesthesia is indeed superior 
to general anaesthesia regarding outcomes of remedial surgery independent of the supposed cause, a second retrospective study was performed. The results are demonstrated in Chapter 7: Chronic postoperative groin pain requiring remedial surgery: Spinal or general anaesthesia? Of note, the study was aimed at investigating a possible association between type of anaesthesia and successful outcome of remedial surgery, rather than identify prognostic factors for success.

The surprising results of these studies have raised the question whether the effect of the anaesthetic technique on results of groin pain surgery is confounded. Consequently, a randomized controlled trial is the appropriate solution. A trial is proposed investigating the true effect of type of anaesthesia and whether confounding factors or effect modifiers possibly are responsible for the effects that were previously found (Chapter 8: Spinal versus general anaesthesia in surgery for inguinodynia (SPINASIA trial): study protocol for a randomized controlled trial).

\section{Case: sequelae from mesh migration}

An important finding of chapter 6 was the suggestion that success after surgery may be determined by the removal of a meshoma, if present. Folding mesh may have a tendency to migrate over time and occasionally entrap organs within the abdominal cavity. An exceptional case is described in Case II. Entrapment of the Appendix in a Meshoma following Inguinal Hernia Repair: a Case Report.

\section{Recall bias}

5. Do recalled pain scores have a significant impact on the effect sizes of retrospective studies on abdominal wall and groin pain?

To address this questions a unique study was performed to analyze a large series of patients to determine the actual bias in effect sizes by using retrospectively obtained pain scores, as presented in Chapter 9: Recall Bias in Pain Scores Evaluating Abdominal Wall and Groin Pain Surgery. 


\section{REFERENCE LIST}

1. Lau WY. History of treatment of groin hernia. World J Surg 2002;26(6):748-59.

2. Read RC. Milestones in the history of hernia surgery: prosthetic repair. Hernia 2004;8(1):814.

3. Czerny V. Studien zur radikalbehandlung der hernien. Wien Med Wochenschr 1877;27:497500.

4. MacEwen W. On the radical cure of oblique inguinal hernia by internal abdominal peritoneal pad, and the restoration of the valved form of the inguinal canal. Ann Surg 1886;4:89.

5. Bassini E. Nuovo metodo per la cura radicale dell'ernia inguinale. Atti Congr Med Ital 1887;2:179-82.

6. Shouldice EE. The treatment of hernia. Ontario Med Rev 1953;20:670-84.

7. McVay CB. Inguinal hernioplasty: common mistakes and pitfalls. Surg Clin North Am 1966;46(5):1089-100.

8. Aquaviva D, Bounet P. Cure d'une volumineuse eventration par plaque de Crinofil. Extraits Bull Soc Chir de Marseille 1944;17.

9. Usher FC, Cogan JE, Lowry TI. A new technique for the repair of inguinal and incisional hernias. Arch Surg 1960;81:847-54.

10. Stoppa R, Petit J, Henry X. Unsutured Dacron prosthesis in groin hernias. Int Surg 1975;60(8):411-2.

11. Lichtenstein IL, Shulman AG, Amid PK, et al. The tension-free hernioplasty. Am J Surg 1989;157(2):188-93.

12. Ger R. The management of certain abdominal herniae by intra-abdominal closure of the neck of the sac. Preliminary communication. Ann R Coll Surg Engl 1982;64(5):342-4.

13. Tran H. Robotic single-port hernia surgery. JSLS 2011;15(3):309-14.

14. Collaboration EUHT. Repair of groin hernia with synthetic mesh: meta-analysis of randomized controlled trials. Ann Surg 2002;235(3):322-32.

15. Grant AM, Scott NW, O'Dwyer PJ. Five-year follow-up of a randomized trial to assess pain and numbness after laparoscopic or open repair of groin hernia. Br J Surg 2004;91(12):157074.

16. Nienhuijs S, Staal E, Strobbe L, et al. Chronic pain after mesh repair of inguinal hernia: a systematic review. Am J Surg 2007;194(3):394-400. 
17. Classification of chronic pain. Descriptions of chronic pain syndromes and definitions of pain terms. Prepared by the International Association for the Study of Pain, Subcommittee on Taxonomy. Pain Suppl 1986;3:S1-226.

18. Bay-Nielsen M, Perkins FM, Kehlet H. Pain and functional impairment 1 year after inguinal herniorrhaphy: a nationwide questionnaire study. Ann Surg 2001;233(1):1-7.

19. Courtney CA, Duffy K, Serpell MG, et al. Outcome of patients with severe chronic pain following repair of groin hernia. Br J Surg 2002;89(10):1310-4.

20. Perkins FM, Kehlet H. Chronic pain as an outcome of surgery. A review of predictive factors. Anesthesiology 2000;93(4):1123-33.

21. Simons MP, Aufenacker T, Bay-Nielsen M, et al. European Hernia Society guidelines on the treatment of inguinal hernia in adult patients. Hernia 2009;13(4):343-403.

22. Koning GG, Wetterslev J, van Laarhoven CJ, et al. The totally extraperitoneal method versus Lichtenstein's technique for inguinal hernia repair: a systematic review with meta-analyses and trial sequential analyses of randomized clinical trials. PloS one 2013;8(1):e52599.

23. Callesen T, Bech K, Kehlet H. Prospective study of chronic pain after groin hernia repair. Br J Surg 1999;86(12):1528-31.

24. Poobalan AS, Bruce J, King PM, et al. Chronic pain and quality of life following open inguinal hernia repair. Br J Surg 2001;88(8):1122-6.

25. Bozuk M, Schuster R, Stewart D, et al. Disability and chronic pain after open mesh and laparoscopic inguinal hernia repair. Am Surg 2003;69(10):839-41.

26. Mikkelsen T, Werner MU, Lassen B, et al. Pain and sensory dysfunction 6 to 12 months after inguinal herniotomy. Anesth Analg 2004;99(1):146-51.

27. Aasvang E, Kehlet H. Chronic postoperative pain: the case of inguinal herniorrhaphy. Br J Anaesth 2005;95(1):69-76.

28. Arregui ME, Navarrete J, Davis CJ, et al. Laparoscopic inguinal herniorrhaphy. Techniques and controversies. Surg Clin North Am 1993;73(3):513-27.

29. McKernan JB, Laws HL. Laparoscopic repair of inguinal hernias using a totally extraperitoneal prosthetic approach. Surg Endosc 1993;7(1):26-8.

30. Do M, Liatsikos E, Beatty J, et al. Laparoendoscopic single-site extraperitoneal inguinal hernia repair: initial experience in 10 patients. J Endourol 2011;25(6):963-8.

31. Engan C, Engan M, Bonilla V, et al. Description of robotically assisted single-site transabdominal preperitoneal (RASS-TAPP) inguinal hernia repair and presentation of clinical outcomes. Hernia 2015;19(3):423-8. 
32. Koning GG, Andeweg CS, Keus F, et al. The transrectus sheath preperitoneal mesh repair for inguinal hernia: technique, rationale, and results of the first 50 cases. Hernia 2012;16(3):2959.

33. Akkersdijk WL, Andeweg CS, Bokkerink WJ, et al. Teaching the transrectus sheath preperiotneal mesh repair: TREPP in 9 steps. Int J Surg 2016;30:150-4.

34. Pelissier EP. Inguinal hernia: preperitoneal placement of a memory-ring patch by anterior approach. Preliminary experience. Hernia 2006;10(3):248-52.

35. Simons MP, Smietanski M, Bonjer HJ, et al. World guidelines for groin hernia management. 2016.

36. Loos MJ, Scheltinga MR, Roumen RM. Surgical management of inguinal neuralgia after a low transverse Pfannenstiel incision. Ann Surg 2008;248(5):880-5.

37. Verhagen T, Roumen RM, Loos MJ, et al. Surgical neurectomy for resistive chronic postPfannenstiel pain syndrome is effective. Submitted 2015.

38. Licheri S, Pisano G, Erdas E, et al. Endometriosis of the round ligament: description of a clinical case and review of the literature. Hernia 2005;9(3):294-7.

39. Hohenfellner R. Nerve injuries in urological surgery. Georgian medical news 2007(143):7-11.

40. Kehlet H, Jensen TS, Woolf CJ. Persistent postsurgical pain: risk factors and prevention. Lancet 2006;367(9522):1618-25.

41. Loos MJ, Roumen RM, Scheltinga MR. Classifying post-herniorrhaphy pain syndromes following elective inguinal hernia repair. World J Surg 2007;31(9):1760-5; discussion 66-7.

42. Amid PK. Causes, prevention, and surgical treatment of postherniorrhaphy neuropathic inguinodynia: triple neurectomy with proximal end implantation. Hernia 2004;8(4):343-9.

43. Chaparro LE, Smith SA, Moore RA, et al. Pharmacotherapy for the prevention of chronic pain after surgery in adults. Cochrane Database Syst Rev 2013;7:CD008307.

44. Baron R. Mechanisms of disease: neuropathic pain--a clinical perspective. Nat Clin Pract Neurol 2006;2(2):95-106.

45. Bouhassira D, Attal N, Alchaar $\mathrm{H}$, et al. Comparison of pain syndromes associated with nervous or somatic lesions and development of a new neuropathic pain diagnostic questionnaire (DN4). Pain 2005;114(1-2):29-36.

46. Amid PK. Radiologic images of meshoma: a new phenomenon causing chronic pain after prosthetic repair of abdominal wall hernias. Arch Surg 2004;139(12):1297-8.

47. Rasmussen PV, Sindrup SH, Jensen TS, et al. Symptoms and signs in patients with suspected neuropathic pain. Pain 2004;110(1-2):461-9. 
48. Burgmans JP, Voorbrood CE, Van Dalen T, et al. Chronic pain after TEP inguinal hernia repair, does MRI reveal a cause? Hernia 2016;20(1):55-62.

49. Lichtenstein IL, Shulman AG, Amid PK, et al. Cause and prevention of postherniorrhaphy neuralgia: a proposed protocol for treatment. Am J Surg 1988;155(6):786-90.

50. Loos MJ, Verhagen T, Scheltinga MR, et al. A randomized controlled trial of injection therapy versus neurectomy for post-herniorrhaphy inguinal neuralgia: rationale and study design. Hernia 2010;14(6):593-7.

51. Kehlet H, Roumen RM, Reinpold W, et al. Invited commentary: Persistent pain after inguinal hernia repair: what do we know and what do we need to know? Hernia 2013;17(3):293-7.

52. Aasvang EK, Kehlet H. Postherniotomy dysejaculation: Successful treatment with mesh removal and nerve transection. Hernia 2008;12(6):645-47.

53. Verhagen T, Loos MJA, Scheltinga MRM, et al. Surgery for chronic inguinodynia following routine herniorrhaphy: beneficial effects on dysejaculation. Hernia 2016;20(1):63-68.

54. Bischoff JM, Linderoth G, Aasvang EK, et al. Dysejaculation after laparoscopic inguinal herniorrhaphy: A nationwide questionnaire study. Surg Endosc 2012;26(4):979-83.

55. Masarani M, Cox R. The aetiology, pathophysiology and management of chronic orchialgia. BJU international 2003;91(5):435-7.

56. Chen DC, Amid PK. Persistent orchialgia after inguinal hernia repair: diagnosis, neuroanatomy, and surgical management: Invited comment to: Role of orchiectomy in severe testicular pain and inguinal hernia surgery: audit of Finnish patient insurance centre. Rönka K, Vironen J, Kokki H, Liukkonen T, Paajanen H. DOI 10.1007/s10029-013-1150-3. Hernia 2015;19(1):61-63.

57. Thomassen I, van Suijlekom JA, van de Gaag A, et al. Ultrasound-guided ilioinguinal/ iliohypogastric nerve blocks for chronic pain after inguinal hernia repair. Hernia 2013;17(3):329-32.

58. Werner MU, Bischoff JM. Persistent postsurgical pain: Evidence from breast cancer surgery, groin hernia repair, and lung cancer surgery, 2014:3-29.

59. Werner MU, Kongsgaard UE. I. Defining persistent post-surgical pain: is an update required? Br J Anaesth 2014;113(1):1-4.

60. Starling JR, Harms BA. Diagnosis and treatment of genitofemoral and ilioinguinal neuralgia. World J Surg 1989;13(5):586-91.

61. Heise CP, Starling JR. Mesh inguinodynia: a new clinical syndrome after inguinal herniorrhaphy? J Am Coll Surg 1998;187(5):514-8. 
62. Amid PK. A 1-stage surgical treatment for postherniorrhaphy neuropathic pain: triple neurectomy and proximal end implantation without mobilization of the cord. Arch Surg 2002;137(1):100-4.

63. Deysine M, Deysine GR, Reed WP, Jr. Groin pain in the absence of hernia: a new syndrome. Hernia 2002;6(2):64-7.

64. Aasvang E, Kehlet H. Surgical management of chronic pain after inguinal hernia repair. Br J Surg 2005;92(7):795-801.

65. Loos MJ, Scheltinga MR, Roumen RM. Tailored neurectomy for treatment of postherniorrhaphy inguinal neuralgia. Surgery 2010;147(2):275-81.

66. Krahenbuhl L, Striffeler H, Baer HU, et al. Retroperitoneal endoscopic neurectomy for nerve entrapment after hernia repair. Br J Surg 1997;84(2):216-9.

67. Giger U, Wente MN, Buchler MW, et al. Endoscopic retroperitoneal neurectomy for chronic pain after groin surgery. Br J Surg 2009;96(9):1076-81.

68. Chen DC, Hiatt JR, Amid PK. Operative management of refractory neuropathic inguinodynia by a laparoscopic retroperitoneal approach. JAMA Surg 2013;148(10):962-7.

69. Lange JF, Kaufmann R, Wijsmuller AR, et al. An international consensus algorithm for management of chronic postoperative inguinal pain. Hernia 2014.

70. Keller JE, Stefanidis D, Dolce CJ, et al. Combined open and laparoscopic approach to chronic pain after inguinal hernia repair. Am Surg 2008;74(8):695-700; discussion 00-1.

71. Koopmann MC, Yamane BH, Starling JR. Long-term follow-up after meshectomy with acellular human dermis repair for postherniorrhaphy inguinodynia. Arch Surg 2011;146(4):427-31.

72. Davis BE, Noble MJ, Weigel JW, et al. Analysis and management of chronic testicular pain. $J$ Urol 1990;143(5):936-9.

73. Choa RG, Swami KS. Testicular denervation. A new surgical procedure for intractable testicular pain. Br J Urol 1992;70(4):417-9.

74. Cadeddu JA, Bishoff JT, Chan DY, et al. Laparoscopic testicular denervation for chronic orchalgia. J Urol 1999;162(3 Pt 1):733-5; discussion 35-6.

75. Reinpold W, Schroeder AD, Schroeder M, et al. Retroperitoneal anatomy of the iliohypogastric, ilioinguinal, genitofemoral, and lateral femoral cutaneous nerve: consequences for prevention and treatment of chronic inguinodynia. Hernia 2015;19(4):539-48.

76. Franneby U, Sandblom G, Nordin P, et al. Risk factors for long-term pain after hernia surgery. Ann Surg 2006;244(2):212-9. 
77. Pierides GA, Paajanen HE, Vironen JH. Factors predicting chronic pain after open mesh based inguinal hernia repair. Int J Surg 2016.

78. Mori T, Souda S, Nezu R, et al. Results of performing mesh plug repair for groin hernias. Surg Today 2001;31(2):129-32.

79. Ozgun H, Kurt MN, Kurt I, et al. Comparison of local, spinal, and general anaesthesia for inguinal herniorrhaphy. Eur J Surg 2002;168(8-9):455-9.

80. Joshi GP, Rawal N, Kehlet H, et al. Evidence-based management of postoperative pain in adults undergoing open inguinal hernia surgery. Br J Surg 2012;99(2):168-85.

81. Alfieri S, Rotondi F, Di Giorgio A, et al. Influence of preservation versus division of ilioinguinal, iliohypogastric, and genital nerves during open mesh herniorrhaphy: prospective multicentric study of chronic pain. Ann Surg 2006;243(4):553-8.

82. Aasvang EK, Gmaehle E, Hansen JB, et al. Predictive risk factors for persistent postherniotomy pain. Anesthesiology 2010;112(4):957-69.

83. Tasmuth T, Estlanderb AM, Kalso E. Effect of present pain and mood on the memory of past postoperative pain in women treated surgically for breast cancer. Pain 1996;68(2-3):343-7.

84. Turk DC, Okifuji A. Perception of traumatic onset, compensation status, and physical findings: impact on pain severity, emotional distress, and disability in chronic pain patients. J Behav Med 1996;19(5):435-53. 



\section{CHAPTER 2}

Randomized clinical trial comparing self-gripping mesh with a standard polypropylene mesh for open inguinal hernia repair

T. Verhagen

W.A.R. Zwaans

M.J.A. Loos

J.A. Charbon

M.R.M. Scheltinga

R.M.H. Roumen

Br J Surg 2016; 103: 812-818 


\section{ABSTRACT}

Background: The introduction of mesh for open inguinal hernia repair has reduced the rate of recurrence, allowing research to focus on prevention of postoperative pain. In an effort to reduce chronic pain, a semiresorbable, lighter and self-gripping mesh was developed.

Methods: A double-blind randomized clinical trial was conducted comparing the self-gripping mesh with a standard polypropylene mesh repair. Patients over 18 years of age undergoing open primary hernia repair were included. Pain was measured on a six-point verbal rating scale (VRS) and a 150-mm visual analogue scale (VAS). Postoperative pain reduction from baseline pain ( $\triangle \mathrm{VAS})$, complications and return to work/hobbies were studied. Data were collected at baseline, 3 weeks, 3 months and 1 year after surgery (primary outcome).

Results: A total of 363 patients were analysed. Median age was 59 (range 19-88) years. Baseline VRS and VAS scores were similar for the two groups. There was no difference in VRS scores at 1-year follow-up. Duration of surgery was significantly shorter with the self-gripping mesh (mean 40 min versus 49 min for standard mesh repair; $P<0.001)$. At 3 weeks, $\triangle$ VAS in patients receiving the self-gripping mesh was significantly larger $(-10.6$ versus -5.0 respectively; $P=0.049)$ and less subjective discomfort was reported $(P=0.016)$. Complication rates, return to work and recurrence rates were similar, although there were more recurrences in the selfgripping mesh group (5.5 versus $2.2 \% ; P=0.103$ ).

Conclusion: A self-gripping mesh for hernia repair may result in less pain in the early postoperative phase but chronic postherniorrhaphy pain is not affected. Recurrence rates may be a potential disadvantage.

Registration number: NTR1212 (www.trialregister.nl) 


\section{INTRODUCTION}

Approximately 30,000 inguinal hernia repairs are done every year in the Netherlands alone. Currently, open mesh techniques are common. The widespread use of this technique has reduced hernia recurrence rates to acceptable levels (less than 2\%), so the focus of scientific attention has shifted towards prevention of postoperative pain ${ }^{1}$. The rate of chronic pain following hernia repair ranges from 11 to $40 \%{ }^{2,3}$.

Chronic pain after hernia repair may occur either through direct nerve damage or by entrapment in scar tissue, or following formation of a meshoma ${ }^{3}$. Although pain may be reduced by meticulous identification of groin nerves, others have studied alternative prosthetic materials. Use of lightweight mesh results in less chronic pain ${ }^{4}$ and diminished foreign body sensation ${ }^{5}$. Modifying fixation material may also lead to attenuation of chronic pain ${ }^{6-13}$.

The Parietene ProGrip ${ }^{\mathrm{Tm}}$ mesh (Covidien, Zurich, Switzerland) was developed to avoid suture fixation and also to diminish the formation of excessive fibrosis during healing. This material is semiresorbable mesh made of large-pore isoelastic polypropylene and is approximately $45 \%$ lighter than standard polypropylene mesh $\left(38 \mathrm{~g} / \mathrm{m}^{2}\right.$ versus $\left.85 \mathrm{~g} / \mathrm{m}^{2}\right)$. Polylactic acid micro-hooks adhering to the surrounding tissue are thought to dissolve over time, resulting in a completely sutureless repair.

One study ${ }^{6}$ using ProGrip ${ }^{\mathrm{Tm}}$ demonstrated that patients reported virtually no pain (median score 0 on a $0-10$ visual analogue scale (VAS)) after 1 year of follow-up. Interim results of a recent multicentre study 13 found that duration of surgery was $6.5 \min (17 \%)$ shorter with the ProGrip ${ }^{\mathrm{Tm}}$ mesh.

A double-blind randomized clinical trial (RCT) was initiated to compare mediumterm postoperative groin pain after ProGrip ${ }^{\mathrm{Tm}}$ or standard polypropylene mesh for hernia repair. 


\section{METHODS}

The study was a single-centre double-blind RCT comparing results of inguinal hernia repair using the ProGrip ${ }^{\text {TM }}$ mesh with a standard sutured Lichtenstein repair using polypropylene mesh. It was conducted at a large Dutch teaching hospital with both junior (year 1-3) and senior (year 4-6) surgical residents. The study followed the Declaration of Helsinki 2004 for ethical research on human subjects, and was approved by the local medical ethics committee (Netherlands Trial Registry number: NTR1212).

\section{Inclusion and exclusion criteria}

All patients aged 18 years or older having an elective open primary inguinal hernia repair with mesh were eligible for the study. Exclusion criteria were: irreducible hernia, infection, presence of a femoral hernia, chronic pain associated with previous locoregional surgery (appendicectomy in right-sided hernias, Pfannenstiel incision, vasectomy) or inability to complete follow-up.

\section{Follow-up}

Before surgery, patients were informed about the nature of the study, and after 2 weeks for consideration they completed an informed consent form. Baseline questionnaires were then obtained concerning patient characteristics, levels of groin pain using a standard VAS (150-mm scale) and a six-point verbal rating scale (VRS), and influence of pain on daily activities.

At 3 weeks after surgery, patients completed a similar questionnaire in the outpatient department investigating return to work, hobbies and household duties. A surgeon blinded to procedure checked wound healing, postoperative complications and somatosensory alterations in the groin. Some 3 months after the operation, the same questionnaires were sent by post. One year after surgery, patients were invited to attend for a physical examination at the outpatient department, followed by completion of the same set of questionnaires.

\section{Primary and secondary outcome measures}

The primary outcome measure was the difference in chronic pain measured on a six-point VRS at 1 year (1, no pain; 2, very mild pain; 3, mild pain; 4, moderate pain; 
5, severe pain; 6, very severe pain). Secondary outcomes were perioperative and early postoperative complications, return to work and differences in hernia recurrence rate.

A difference in pain between preoperative and postoperative values ( $\triangle$ VAS) was calculated at all follow-up time points.

\section{Blinding, stratification and randomization}

Just before the operation, the operating surgeon asked the trial secretary to open an envelope containing a trial registration number. This ensured anonymity and randomized for type of mesh to be used (ProGrip ${ }^{\text {Ts }}$ or standard polypropylene). Randomization occurred in blocks of eight. Patients were free to choose the type of anaesthesia (general or locoregional), but randomization was prestratified into these two groups to prevent confounding. Both patients and surgeons in the outpatient department were blinded to the type of mesh used.

\section{Operative technique}

An 8-10-cm skin incision just cranial to the inguinal ligament allowed identification of the fascia of the external oblique muscle. This was opened, followed by dissection and repositioning of the hernial sac into the abdominal cavity. A medial-type hernia was redressed using a running stitch. In the self-gripping mesh group, the prefabricated neoannulus of the mesh was wrapped around the spermatic cord and placed beneath the fascia of the external oblique muscle. Overlap on the pubic tubercle and the inguinal ligament was at least $1 \mathrm{~cm}$. The fascia of the external oblique was then closed over the mesh using a running stitch. Subcutaneous tissue and skin were closed with absorbable sutures. In the control group, the polypropylene mesh was secured as described by Lichtenstein ${ }^{14}$.

The surgeon was instructed to identify the ilioinguinal nerve, the iliohypogastric nerve and, if possible, the genital branch of the genitofemoral nerve. A nerve was resected prophylactically if it was interfering with proper mesh positioning. Immediately after surgery a questionnaire evaluating the type of anaesthesia, duration of surgery (skinto-skin), handling of the hernia and its characteristics, nerve identification and possible resection, perioperative complications, and the quality of the mesh fixation was completed by the resident or surgeon. This case record form was stored in a separate file. Mesh specifics were omitted from the official operative notes. 
All operations were performed or supervised by surgeons or trainees who were experienced in both Lichtenstein and self-gripping mesh techniques. Before allowing participation in the study, each surgeon or resident performed at least three self-gripping mesh hernia repairs under the guidance of an experienced surgeon. Both junior and senior surgical trainees operated under supervision of a trained surgeon.

\section{Statistical analysis}

The hypothesis was that the reported rate of chronic pain of any intensity (40\%) could be reduced to $25 \%$ in the self-gripping mesh group. Assuming a type I error of 0.05 and a type II error of $0 \cdot 2$, it was calculated that two samples of 165 patients each would be required. Expecting a 5\% loss to follow-up, 175 patients were included in each study arm. Analysis was done with SPSS ${ }^{\circledast}$ version 17.0 (IBM, Armonk, New York, USA), with $\chi 2$ tests performed on nominal variables and Mann-Whitney $U$ tests on ordinal variables. $P<0.05$ was considered statistically significant.

\section{RESULTS}

A total of 373 patients consented in writing to participate in the study (Figure 2.1). Five patients were excluded before randomization (cancelled operation, 4; withdrew consent, 1); therefore 368 patients were randomized. Five further patients were excluded because of a protocol violation (local anaesthesia, 3; recurrent hernia, 1; loss of data set, 1); 363 patients were analysed. At the 3-week control, 360 patients (99.2\%) were evaluated. A total of 348 patients (96.7\%) completed the 3-month follow-up. At 1 year, data were obtained from 338 patients (93.1\%). 


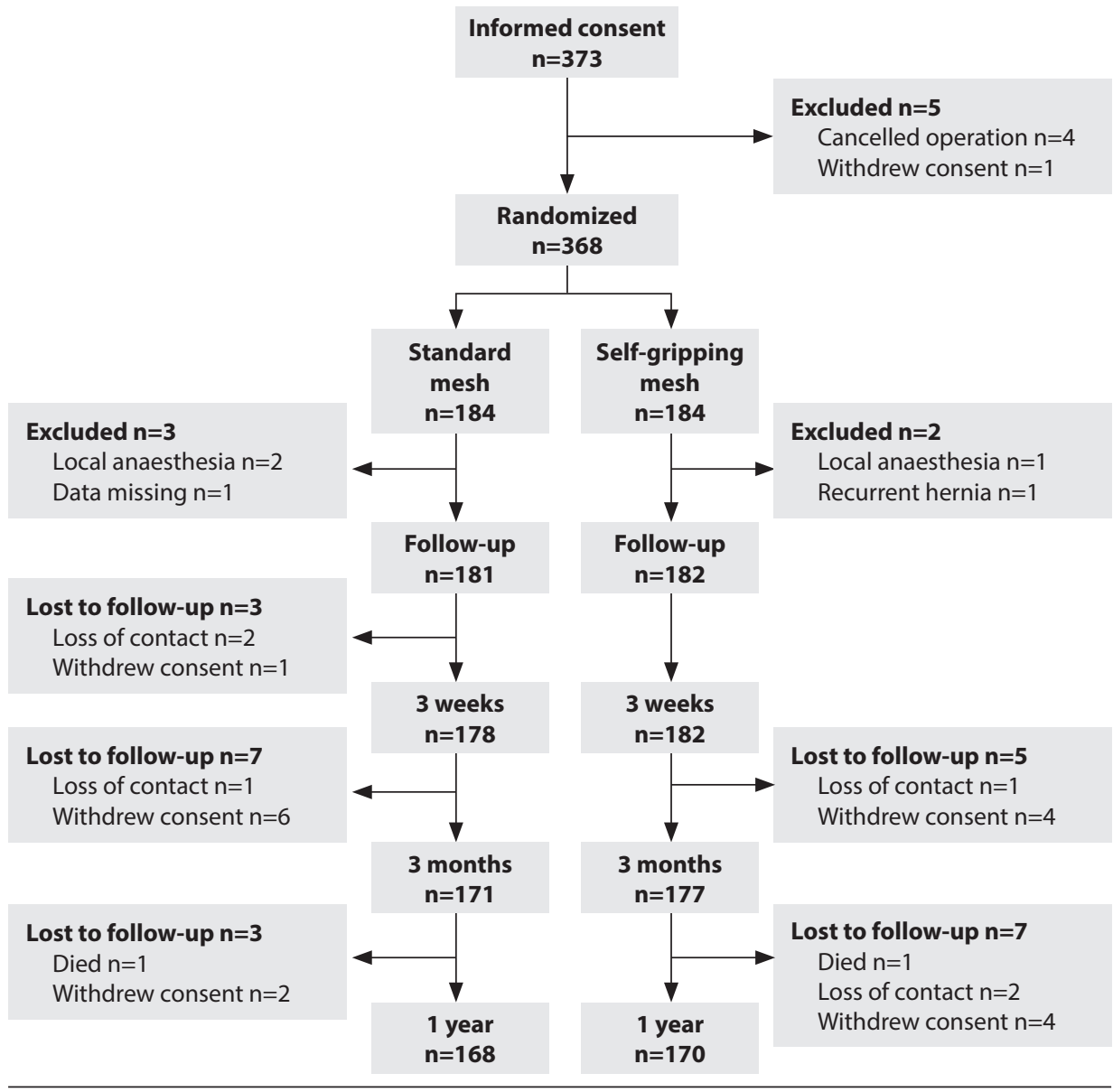

\section{FIGURE 2.1:}

Flowchart depicting numbers of patients during different stages of the study with reasons for loss to follow-up.

\section{Demographics}

Of the 363 analysed patients, 354 (97.5\%) were men. Median age was 59 (range 1988) years. A total 182 patients were randomized to receive the self-gripping mesh, and 181 were allocated to the standard mesh.

Risk factors for inguinal hernia were distributed evenly between study groups, as was the presence of other chronic pain syndromes (low back pain, headache, complex regional pain syndrome). Most patients were healthy, with American Society of Anesthesiologists (ASA) grade I or II (96.4\%); the remaining 3.6\% were ASA grade III (Table 2.1). 
TABLE 2.1:

Demographic details for the study population

\begin{tabular}{|c|c|c|}
\hline & $\begin{array}{l}\text { Standard mesh } \\
(n=181)\end{array}$ & $\begin{array}{l}\text { Self-gripping mesh } \\
(\mathrm{n}=182)\end{array}$ \\
\hline Age (years)* & $58(19-86)$ & $60(20-88)$ \\
\hline Sex ratio (M: F) & $179: 2$ & $175: 7$ \\
\hline Body mass index $\left(\mathrm{kg} / \mathrm{m}^{2}\right)^{*}$ & $25(19-36)$ & $25(18-33)$ \\
\hline \multicolumn{3}{|l|}{ Hernia side } \\
\hline Right & 98 & 102 \\
\hline Left & 83 & 80 \\
\hline \multicolumn{3}{|l|}{ Symptomatic hernia } \\
\hline Yes & 168 & 157 \\
\hline No & 13 & 25 \\
\hline Weeks of complaint* & $4(1-216)$ & $3(1-120)$ \\
\hline \multicolumn{3}{|l|}{ Other chronic pain } \\
\hline Low back pain & 10 & 16 \\
\hline Headache & 4 & 4 \\
\hline Regional pain syndrome & 2 & 1 \\
\hline \multicolumn{3}{|l|}{ Risk factors } \\
\hline No risk factors & 85 & 78 \\
\hline COPD & 7 & 10 \\
\hline Constipation & 6 & 9 \\
\hline $\mathrm{BPH}$ & 13 & 20 \\
\hline Obesity & 5 & 6 \\
\hline Smoking & 36 & 41 \\
\hline Heavy manual labour & 53 & 45 \\
\hline Aortic aneurysm & 2 & 7 \\
\hline \multicolumn{3}{|l|}{ Work situation } \\
\hline Contract & 77 & 86 \\
\hline Independent & 20 & 11 \\
\hline Retired & 74 & 77 \\
\hline Unemployed & 4 & 3 \\
\hline Disabled & 4 & 4 \\
\hline \multicolumn{3}{|l|}{ ASA fitness grade } \\
\hline 1 & 115 & 113 \\
\hline II & 45 & 50 \\
\hline III & 8 & 3 \\
\hline
\end{tabular}

* Values are median (range). Data were missing for some patients in various categories. COPD, chronic obstructive pulmonary disease; BPH, benign prostatic hyperplasia; ASA, American Society of Anesthesiologists. 


\section{Hernia characteristics}

There were slightly more right-sided hernias (200 versus 163 on the left side). The mean duration of symptoms before surgical intervention was 3 (range 0-60) months. Just 38 hernias were asymptomatic (10.5\%). Most hernias were either medial or lateral, but 85 (23.4\%) were found to have both a lateral and a medial component.

\section{Surgical characteristics}

A total of 277 procedures (76.3\%) were done by junior trainees under supervision, 63 (17.4\%) were done by a senior trainee alone, and $23(6.3 \%)$ by a trained surgeon. There was an even distribution among study groups with respect to grade of surgeon. In the self-gripping mesh group, surgery was done under general anaesthesia in 49 patients, whereas 133 received a locoregional anaesthetic technique. These numbers were similar in the standard mesh group (42 and 139).

Although the protocol dictated routine ilioinguinal nerve identification, this was completed successfully in only 291 patients $(80.2 \%)$. The iliohypogastric nerve and the genital branch of the genitofemoral nerve were identified in 154 (42.4\%) and 179 (49.3\%) of the patients respectively. Each nerve possibly interfering with proper mesh positioning was resected, in accordance with the protocol. Accidental nerve resections were also recorded. In total, 41 iliohypogastric nerves (11.3\%), 86 ilioinguinal nerves (23.7\%) and 36 genital branches of the genitofemoral nerve (9.9\%) were resected prophylactically. Accidental resection occurred of one iliohypogastric and six ilioinguinal nerves. There was no significant difference between the study groups. Mean duration of surgery was $40 \mathrm{~min}$ using the self-gripping mesh and $49 \mathrm{~min}$ in the standard sutured mesh group $(P<0.001)$.

\section{Primary outcome and pain scores}

Before surgery, pain levels were similar in the two groups (ProGrip ${ }^{\text {ma }} 2.8$ versus standard mesh 2.7; $P=0.330$ ). There was no significant difference between the two procedures with regard to the primary outcome of the study (pain on VRS after 1 year): scores 1.3 and 1.2 respectively $(P=0.950)$ (Figure $2.2 \mathrm{a})$.

Patients who had received a self-gripping mesh had less pain during routine activities at the 3-week follow-up ( $P=0.060)$ (Figure 2.2b). Moreover, they reported significantly less discomfort as measured on a VAS $(P=0.016)$ (Figure $2.2 \mathrm{c})$. By the 3 -month and 1-year follow-ups, these differences had disappeared. 
When comparing $\triangle$ VAS scores after 3 weeks, a significantly greater reduction in pain level during routine activities was recorded in the self-gripping mesh group (10.6 (95\% CI 5.1 to 16.0 ) versus 5.0 ( -0.9 to 10.9 ) with the standard mesh; $P=0.049$ ) (Figure 2.2b). However, $\triangle$ VAS scores during routine activities at other time points were similar for the two procedures, as were the $\triangle$ VAS scores concerning pain in resting conditions and during strenuous activities.

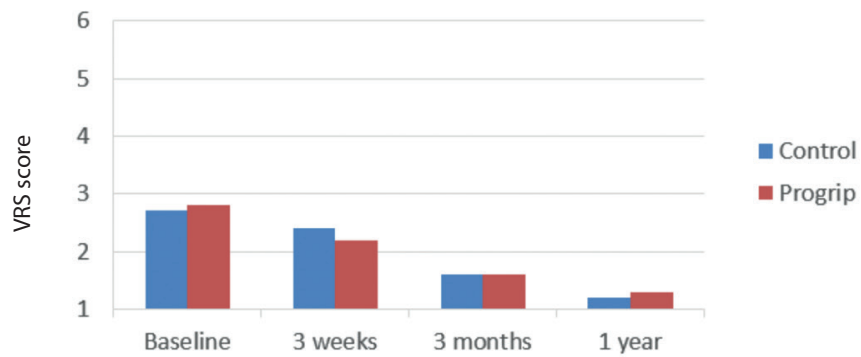

A VRS scores

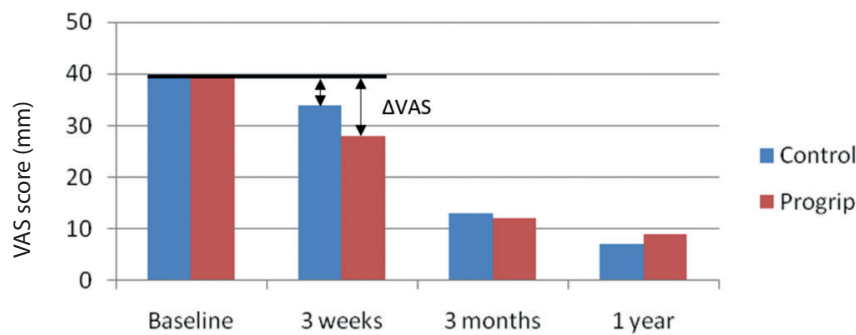

B VAS scores during routine activities

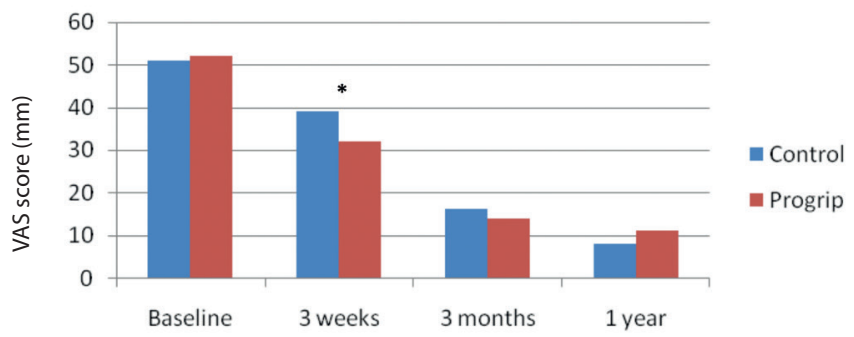

C VAS scores for subjective discomfort

\section{FIGURE 2.2:}

a Verbal rating scale (VRS) and b,c visual analogue scale (VAS) scores for routine activities (b) and subjective discomfort (c) in self-gripping and standard mesh groups. $b$ The reduction from baseline scores during routine activities ( $\triangle V A S$ ) is also shown. $c * P=0.016$ (Mann-Whitney $U$ test). 


\section{Secondary outcomes}

There were no significant differences between the two procedures with regard to the secondary outcomes of the study.

Early and late complication rates were similar. Twenty-five patients in the selfgripping mesh group developed complications (seroma, 4; haematoma, 12: superficial wound infection, 3 ; deep wound infection, 1; urinary tract infection, 1 ; urinary retention, 2; epididymitis, 1; ischaemic orchitis, 1) compared with 29 in the standard mesh group (seroma, 2; haematoma, 17; wound infection, 6; urinary retention, 4).

At the 3-week follow-up, 66.5\% (121 of 182) of patients in the self-gripping mesh group had returned to work versus $74.7 \%$ (133 of 178 ) in the standard mesh group $(P=0.240)$. At the 1 -year follow-up this was $97 \%$ ( 88 of 91 ) versus $95 \%$ ( 88 of 93 ) respectively $(P=0.350$ ). The proportions of patients who were able to do their hobbies or household duties were also similar.

At baseline, 55 (30.2\%) of the 182 patients in the self-gripping mesh group reported impairment of daily activities because of their hernia, compared with 45 (24.9\%) of the 181 in the standard mesh group. At 3 weeks these proportions were 19.2\% (35 of 182) and $23.0 \%$ (41 of 178 ) respectively $(P=0.116)$, and at 1 year $8.8 \%$ ( 15 of 170$)$ and $7.1 \%(12$ of 168) $(P=0.279)$. After 1 year, ten hernia recurrences (5.5\%) had been observed in the self-gripping mesh group, compared with four (2.2\%) with the standard mesh $(P=0.103)$.

\section{DISCUSSION}

Mesh placement is common practice in open inguinal hernia surgery. The development of chronic pain is of ongoing concern, as its onset is unpredictable. Chronic pain rates vary from 11 to $40 \%$, depending on definition ${ }^{2,3}$. The aim of the present study was to compare pain levels in patients undergoing open hernia repair using two different meshes. Preliminary results of an earlier randomized study ${ }^{15}$ found that receiving a ProGrip ${ }^{\mathrm{Tm}}$ mesh resulted in increased levels of pain. In contrast, others ${ }^{13}$ found beneficial effects of ProGrip ${ }^{\text {Ts }}$ mesh placement on pain. In both studies, the differences were significant only during the early postoperative phase, and disappeared at later follow-up.

In the present study, preoperative pain scores were similar, which was crucial as preoperative pain may be a risk factor for chronic postoperative pain ${ }^{16}$. At the different 
time points during follow-up there was no significant difference in pain intensity using the VRS between the two groups. On the VAS, however, pain intensity at 3 weeks showed a trend in favour of the self-gripping mesh, with less pain during routine activities $(P=0.060)$ and a reduced subjective feeling of discomfort $(P=0.016)$. Similarly, $\triangle$ VAS scores after 3 weeks also showed a significantly greater pain reduction compared with preoperative levels when the self-gripping mesh was used. Similar effects on this $\triangle$ VAS in the early postoperative phase have been published previously ${ }^{13}$. At later time points, however, differences in $\triangle$ VAS disappeared. The ProGrip ${ }^{\text {ma }}$ mesh may therefore attenuate early postoperative pain, but has no effect on chronic groin pain.

The present study also found other benefits associated with the self-gripping mesh. The duration of operation was significantly shorter (40 versus $49 \mathrm{~min}$ ). Other authors $^{13,15,17}$ have found similar shorter operation times with the self-gripping mesh.

Return to work is considered an important outcome measure; less postoperative pain could lead to earlier return to work ${ }^{18}$; however, the present study found no difference between the mesh types. Early and late complication rates (which affect return to work) were similar, and at least two-thirds of patients in both groups had resumed work after 3 weeks. After 1 year, nearly all previously employed patients had returned to work.

Potential advantages of a self-gripping mesh need to be balanced against a possible disadvantage of higher recurrence rates in the long term. Other ProGrip ${ }^{\text {Tw }}$ studies $^{6,15,18}$ have shown recurrence rates of up to $2 \%$ after 1 year. Unlike these, the present study protocol did not allow for a medial stitch to fix the mesh, which has been shown to result in more postoperative pain $^{13}$. Here, the difference in 1 -year recurrence rates $(5.5 \%$ for the self-gripping mesh versus $2.2 \%$ for the standard mesh) was not significant $(P=0.103)$. However, the trend towards a higher recurrence rate for ProGrip ${ }^{\mathrm{Tw}}$ suggests further followup is important to clarify the issue of recurrence.

The present study has limitations. A teaching hospital setting with trainee surgeons may be suboptimal as expert surgeons may attain superior results. However, the present study environment is representative of daily practice, allowing better generalization of the results. There was a high identification rate of all three relevant inguinal nerves in both groups, an approach that requires more extensive dissection but that may reduce the rate of chronic groin pain ${ }^{19}$. These extra efforts were taken because of the trial protocol, but may not reflect standard practice in the Netherlands $\mathrm{s}^{20,21}$. 
Self-gripping mesh is a viable and safe option for inguinal hernia repair. There was a trend towards less early postoperative pain, and the surgery was quicker, even in relatively inexperienced hands. However, there was no effect on the rate of groin pain after 3 months, and a potentially increased risk of recurrence that requires further follow-up.

\section{Acknowledgements}

The authors received a grant from Covidien before the study started. The authors retained the right to publish the study results. Although Covidien had the right to review the data, they played no part in writing the manuscript. 


\section{REFERENCE LIST}

1. Nienhuijs SW, van Oort I, Keemers-Gels ME, et al. Randomized trial comparing the Prolene Hernia System, mesh plug repair and Lichtenstein method for open inguinal hernia repair. Br J Surg 2005;92(1):33-8.

2. Nienhuijs S, Staal E, Strobbe L, et al. Chronic pain after mesh repair of inguinal hernia: a systematic review. Am J Surg 2007;194(3):394-400.

3. Loos MJ, Roumen RM, Scheltinga MR. Chronic sequelae of common elective groin hernia repair. Hernia 2007;11(2):169-73.

4. O’Dwyer PJ, Kingsnorth AN, Molloy RG, et al. Randomized clinical trial assessing impact of a lightweight or heavyweight mesh on chronic pain after inguinal hernia repair. Br J Surg 2005;92(2):166-70.

5. Post S, Weiss B, Willer M, et al. Randomized clinical trial of lightweight composite mesh for Lichtenstein inguinal hernia repair. Br J Surg 2004;91(1):44-8.

6. Chastan P. Tension-free open hernia repair using an innovative self-gripping semi-resorbable mesh. Hernia 2009;13(2):137-42.

7. Champault G, Torcivia A, Paolino L, et al. A self-adhering mesh for inguinal hernia repair: preliminary results of a prospective, multicenter study. Hernia 2011;15(6):635-41.

8. Paajanen H, Kossi J, Silvasti S, et al. Randomized clinical trial of tissue glue versus absorbable sutures for mesh fixation in local anaesthetic Lichtenstein hernia repair. Br J Surg 2011;98(9):1245-51.

9. Kim-Fuchs C, Angst E, Vorburger S, et al. Prospective randomized trial comparing sutured with sutureless mesh fixation for Lichtenstein hernia repair: long-term results. Hernia 2012;16(1):21-7.

10. Campanelli G, Pascual MH, Hoeferlin A, et al. Randomized, controlled, blinded trial of Tisseel/Tissucol for mesh fixation in patients undergoing Lichtenstein technique for primary inguinal hernia repair: results of the TIMELI trial. Ann Surg 2012;255(4):650-7.

11. Canonico S, Benevento R, Perna G, et al. Sutureless fixation with fibrin glue of lightweight mesh in open inguinal hernia repair: effect on postoperative pain: a double-blind, randomized trial versus standard heavyweight mesh. Surgery 2013;153(1):126-30.

12. Ladwa N, Sajid MS, Sains P, et al. Suture mesh fixation versus glue mesh fixation in open inguinal hernia repair: a systematic review and meta-analysis. Int J Surg 2013;11(2):128-35. 
13. Kingsnorth A, Gingell-Littlejohn M, Nienhuijs S, et al. Randomized controlled multicenter international clinical trial of self-gripping Parietex ProGrip polyester mesh versus lightweight polypropylene mesh in open inguinal hernia repair: interim results at 3 months. Hernia 2012;16(3):287-94.

14. Amid PK. Lichtenstein tension-free hernioplasty: its inception, evolution, and principles. Hernia 2004;8(1):1-7.

15. Jorgensen LN, Sommer T, Assaadzadeh S, et al. Randomized clinical trial of self-gripping mesh versus sutured mesh for Lichtenstein hernia repair. Br J Surg 2013;100(4):474-81.

16. Aasvang E, Kehlet $\mathrm{H}$. Chronic postoperative pain: the case of inguinal herniorrhaphy. $\mathrm{Br} J$ Anaesth 2005;95(1):69-76.

17. Anadol AZ, Akin M, Kurukahvecioglu O, et al. A prospective comparative study of the efficacy of conventional Lichtenstein versus self-adhesive mesh repair for inguinal hernia. Surg Today 2011;41(11):1498-503.

18. Quyn AJ, Weatherhead KM, Daniel T. Chronic pain after open inguinal hernia surgery: suture fixation versus self-adhesive mesh repair. Langenbecks Arch Surg 2012;397(8):1215-8.

19. Smeds S, Lofstrom L, Eriksson O. Influence of nerve identification and the resection of nerves 'at risk' on postoperative pain in open inguinal hernia repair. Hernia 2010;14(3):265-70.

20. Lange JF, Wijsmuller AR, van Geldere D, et al. Feasibility study of three-nerve-recognizing Lichtenstein procedure for inguinal hernia. Br J Surg 2009;96(10):1210-4.

21. Wijsmuller AR, Lange JF, van Geldere D, et al. Surgical techniques preventing chronic pain after Lichtenstein hernia repair: state-of-the-art vs daily practice in the Netherlands. Hernia 2007;11(2):147-51. 



\section{CHAPTER 3}

Groin pain characteristics and recurrence rates: Long-term results of a randomized controlled trial comparing self-gripping Progrip mesh and sutured polypropylene mesh for open inguinal hernia repair

W.A.R. Zwaans

T. Verhagen

L. Wouters

M.J.A. Loos

R.M.H. Roumiten

M.R.M. Scheltinga

Ann Surg 2017 [Epub ahead of print]

Awarded with 'Best Clinical Research Award' by the European Society for Surgical Research 


\section{ABSTRACT}

Background: Some patients with an inguinal hernia develop chronic pain following open mesh insertion. Previous trials comparing a semi-resorbable, self-gripping Progrip $^{\text {Tw }}$ mesh with a standard sutured polypropylene mesh found conflicting results regarding recurrence rates and residual groin pain. The present study investigates long-term groin pain and inguinal hernia recurrence rates of two types of mesh and to describe the evolution of postoperative groin sensory disturbances.

Methods: Patients $>18$ years scheduled for open primary hernia repair were randomized to a self-gripping mesh (Progrip ${ }^{\mathrm{Tm}}$ ) or a polypropylene mesh (standard). Removal of the inguinal nerves was left to the discretion of the surgeon. Pain was measured using VAS over a three year period. Pain characteristics and hernia recurrences were determined using physical examination.

Results: Data of 274 patients were complete (75\% three-year follow-up rate). Pain steadily decreased over time in both groups in a similar fashion (moderate pain 3.7\% in each group). Hyperesthesia was experienced by $2.2 \%$ and $3.7 \%$ and hypoesthesia in $12 \%$ and $19 \%$ in Progrip ${ }^{\mathrm{Tm}}$ and standard group respectively. One of seven Progrip ${ }^{\mathrm{Tm}}$ patients reported a foreign body feeling versus one of five standard patients $(p=0.06)$. Altered skin sensations were not related to a neurectomy. Hernia recurrence rate was $11.5 \%$ in the Progrip ${ }^{\text {tw }}$ and 5\% in the standard group $(\mathrm{p}=0.05)$.

Conclusion: Three years after insertion of a self-gripping Progrip ${ }^{\mathrm{Tm}}$ mesh or a sutured polypropylene mesh for an open primary inguinal hernia repair, groin pain is minimal although altered groin skin sensations and foreign body feeling are quite common. A Progrip ${ }^{\mathrm{Tw}}$ hernia repair is associated with a high recurrence rate. 


\section{INTRODUCTION}

Ever since prosthetic meshes were introduced for inguinal hernia surgery in $1958^{1}$, hernia recurrence rates have dropped drastically. However, the search for the optimal mesh is ongoing as the use of foreign body material is potentially associated with longlasting postoperative pain in some patients. Nowadays, chronic ( $>3$ months) groin pain ('postherniorrhaphy inguinodynia') is the most clinically relevant complication following open hernia repair and poses a major health issue whereas progress of effective therapeutic options is slow. Therefore, prevention is crucial.

A self-gripping mesh was introduced that was claimed to potentially prevent the onset of chronic pain. A self-gripping mechanism is hypothetically superior as sutures, a potential cause of ongoing pain due to nerve damage, are not required ${ }^{2}$. Recent studies indeed demonstrated short-term ( $\leq 1$ year postoperatively) beneficial effects of selfgripping meshes when compared to standard suturing mesh techniques ${ }^{3-5}$. Regardless of mesh type or technique, groin pain is known to diminish over time $e^{6,7}$. More solid follow-up data regarding inguinodynia on the longer term are required. A recent European Hernia Society recommendation advised a minimal three years follow-up for determining long-term-outcome for pain $^{8}$. This time period is also relevant for a more appropriate determination of recurrence rate as more hernia recurrences following self-gripping meshes were reported by two studies ${ }^{3,9}$. At present therefore, insufficient evidence is available favouring self-gripping meshes over standard sutured meshes ${ }^{4}$.

A recent randomized trial comparing a semi-resorbable, lighter and self-gripping Progrip $^{\mathrm{Tm}}$ mesh with a standard sutured polypropylene mesh found that short-term postoperative pain was attenuated following the Progrip ${ }^{\mathrm{mx}}$ mesh $^{9}$. Aim of the present study is to report on pain intensity and recurrence rates at three years. Characteristics and course of postherniorrhaphy pain over time are described possibly allowing for improved counselling regarding symptom severity following two types of inguinal hernia repair. 


\section{METHODS}

\section{Trial design}

Details of study protocol and one year results of this double blind, randomized controlled trial (RCT) that was conducted in a single hospital in the Netherlands were published previously ${ }^{9}$. Patient enrolment started in March, 2008 and ended in October 2010. The trial followed guidelines of the declaration of Helsinki (version 2004) and was approved by the Medical Ethics Committee of Máxima Medical Centre (Veldhoven, the Netherlands). The study protocol was registered at www.trialregister.nl (NTR identification number 1212) and the present paper was written according to the CONSORT 2010 statement for reporting randomized trials ${ }^{10}$. Analysis of data was performed between August 2016 and November 2016.

\section{Participants and interventions}

Patients over 18 years of age undergoing open primary inguinal hernia repair were studied. Exclusion criteria were physical signs of infection, presence of a femoral or incarcerated hernia, chronic groin pain associated with earlier surgery (e.g. caesarean section, appendectomy, vasectomy) or inability to complete follow-up (cognitive impairment, language barrier, lacking permanent address). Patients were assigned in a 1:1 allocation ratio receiving Progrip ${ }^{\mathrm{Tm}}$ (Covidien, Dublin, Ireland) or a control group undergoing conventional polypropylene sutured Lichtenstein repair as previously described ${ }^{9}$. All operations were executed in the teaching hospital MMC Veldhoven/ Eindhoven in a day care setting. Surgeons were instructed to include specifics of the surgery including nerve identification and handling in the operative notes. Removal of interfering inguinal nerves (iliohypogastric, ilioinguinal or genitofemoral) was left to the discretion of the surgeon. Fixation sutures were not used in the Progrip ${ }^{\mathrm{m}}$ mesh as recommended by the manufacturer. The Progrip ${ }^{\mathrm{Tm}}$ mesh is a biocomponent hydrophilic monofilament polyester mesh with resorbable polylactic acid micro-grips. After resorption of this self-gripping system, the remaining mesh has a $38 \mathrm{~g} \mathrm{~m}^{-2}$ density. In contrast, conventional monofilament non-resorbable polypropylene mesh, having a $85 \mathrm{~g}$ $\mathrm{m}^{-2}$ density, requires sutures for fixation. 


\section{Outcomes}

Collection of data was performed during timed outpatient controls with physical examinations at three weeks, three months, one year and three years after hernia repair. The primary outcome measure was a potential difference in pain intensity between the Progrip $^{\text {tw }}$ and the standard mesh group as determined by a Visual Analogue Scale (VAS). At each of these follow-up points a patient was instructed to complete a form including four different scores regarding pain levels in the preceding week: highest VAS score, VAS score at rest, VAS during daily activities and VAS during heavy work. In addition, characteristics of neuropathic (hypoesthesia, hyperesthesia, allodynia) and nociceptive pain (foreign body feeling, periostitis, bursitis, funiculodynia) as well as sexual functioning were determined using interviews, questionnaires and physical examination. The secondary outcome measure was difference in hernia recurrence rate.

Physical examinations were focused on distinguishing pain types and on assessing a potential recurrence. An indication of a neuropathic pain syndrome is the presence of a point of maximum pain with radiation towards the associated skin area of the involved inguinal nerve. The point of maximum pain is usually covered by an area of altered skin sensation exhibiting positive symptoms (hyperalgesia, allodynia or hyperpathia), or negative symptoms (hypoesthesia or hypoalgesia) ${ }^{11}$. Skin pinching, a swab or an alcohol soaked gauze may aid in discriminating these neuropathic symptoms, as previously described $^{12}$. Painful palpation among the pubic tubercle may reflect a periostitis. In patients with adductor tendinitis, palpation and provocation of the origin of the adductor magnus muscle is prominent.

\section{Statistics}

Specifics of calculation of sample size and randomization were previously published ${ }^{9}$. Analysis was performed using SPSS version 22.0 (SPSS Inc. ${ }^{\circledR}$ ) for Windows. Baseline group differences were checked for random distribution by the independent t-test and $\chi^{2}$ test for normally distributed and categorical variables. Group differences in pain were analyzed by a repeated measure analysis allowing a comparison of the overall patient pain experience over time. Recurrence rates and changes in characteristics of groin pain and sensibility were compared by a $\chi^{2}$ test. A $p<0.05$ group difference was considered statistically significant. Potential correlations between groin skin hypoesthesia or hyperesthesia and a previous neurectomy were calculated using the Spearman correlation coefficient. 


\section{RESULTS}

\section{Participant flow and numbers analyzed}

A total of 368 patients were randomized and 274 patients reached the three year study end point (75\% follow-up rate). A CONSORT flowchart illustrates number of participants and reasons for withdrawal at each study stage (figure 3.1).

\section{CONSORT

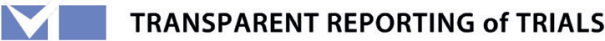

Enrollment

Assessed for eligibility $(n=373)$

\section{Excluded $(\mathbf{n}=5)$}

$\rightarrow$ - Not meeting inclusion criteria $(n=4)$

- Declined to participate $(n=1)$

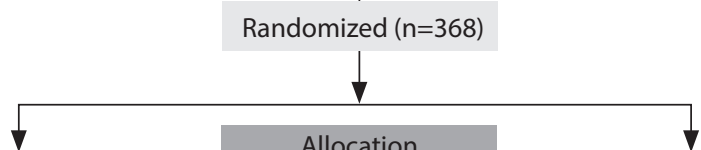

\begin{tabular}{|c|c|}
\hline $\begin{array}{l}\text { Allocated to Progrip mesh }(n=184) \\
\text { - Received allocated intervention }(n=182) \\
\text { - Did not receive allocated intervention } \\
\text { - Local anaesthesia }(n=1) \\
\text { - Recurrent hernia }(n=1)\end{array}$ & $\begin{array}{l}\text { Allocated to conventional mesh }(n=184) \\
\text { - Received allocated intervention }(n=181) \\
\text { - Did not receive allocated intervention } \\
\text { - Local anaesthesia }(n=2) \\
\text { - Loss of data set }(n=1)\end{array}$ \\
\hline \multicolumn{2}{|c|}{ Follow-Up 1 year } \\
\hline $\begin{array}{l}\text { Included in primary analysis }(n=170) \\
\text { - Lost to follow-up }(n=3) \\
\text { - Withdrew informed consent }(n=8) \\
\text { - Deceased }(n=1)\end{array}$ & $\begin{array}{l}\text { Included in primary analysis }(n=168) \\
\text { - Lost to follow-up }(n=3) \\
\text { - Withdrew informed consent }(n=9) \\
\text { - Deceased }(n=1)\end{array}$ \\
\hline \multicolumn{2}{|c|}{ Follow-Up 3 years } \\
\hline $\begin{array}{l}\text { Loss-to-follow-up }(n=31) \\
\text { - Lost to follow-up }(n=15) \\
\text { - Withdrew informed consent }(n=10) \\
\text { - Cognitive impairment }(n=1) \\
\text { - Deceased }(n=5)\end{array}$ & $\begin{array}{l}\text { Loss-to-follow-up }(n=33) \\
\text { - Lost to follow-up }(n=14) \\
\text { - Withdrew informed consent }(n=14) \\
\text { - Deceased }(n=5)\end{array}$ \\
\hline$\nabla$ & $\nabla$ \\
\hline $\begin{array}{l}\text { Analysed ( } n=139) \\
\text { - Excluded from analysis }(n=0)\end{array}$ & $\begin{array}{l}\text { Analysed }(n=135) \\
\text { - Excluded from analysis }(n=0)\end{array}$ \\
\hline
\end{tabular}

FIGURE 3.1:

CONSORT flow diagram for reporting trials. 


\section{Baseline characteristics}

Patient characteristics, rates of inguinal nerves identification or rates of resection were not different between groups (table 3.1). Previous studies have identified the resection of nerves as potential risk factors for the onset of chronic postoperative pain ${ }^{8,13,14}$.

TABLE 3.1:

Characteristics of patients three years after receiving a Progrip ${ }^{\mathrm{TM}}$ mesh or a polypropylene mesh for primary inguinal hernia repair.

\begin{tabular}{|c|c|c|c|c|}
\hline & $\begin{array}{l}\text { Total group } \\
(n=274)\end{array}$ & $\begin{array}{l}\text { Progrip }^{\mathrm{TM}} \\
(\mathrm{n}=139)\end{array}$ & $\begin{array}{l}\text { Standard } \\
(\mathrm{n}=135)\end{array}$ & p-value \\
\hline Age & $60 \pm 15$ & $61 \pm 14$ & $59 \pm 16$ & 0.42 \\
\hline Gender (M:F) & $271: 3$ & $138: 1$ & $133: 2$ & 0.54 \\
\hline Body Mass Index $\left(\mathrm{kg} \mathrm{m}^{-2}\right)$ & $25 \pm 3$ & $25 \pm 5$ & $25 \pm 3$ & 0.12 \\
\hline $\begin{array}{l}\text { Duration of complaints before hernia repair } \\
\text { (months) }\end{array}$ & $3(1-6)$ & $3(1-6)$ & $3(2-6)$ & 0.53 \\
\hline VAS* before operation $(0-100)$ & $15(5-33)$ & $14(5-35)$ & $16(5-32)$ & 0.47 \\
\hline \multicolumn{5}{|l|}{ Pain syndromes before hernia repair (\%) } \\
\hline Lower back pain & 6.9 & 9.4 & 4.4 & 0.11 \\
\hline CRPS‡ & 0.7 & 0 & 1.5 & 0.15 \\
\hline Chronic headache & 2.2 & 2.2 & 2.2 & 0.97 \\
\hline \multicolumn{5}{|l|}{ Type of anaesthesia during hernia repair (\%) } \\
\hline General & 22 & 24 & 19 & 0.29 \\
\hline Spinal & 78 & 76 & 81 & \\
\hline \multicolumn{5}{|l|}{ Intraoperative nerve identification (\%) } \\
\hline Ilioinguinal & 83 & 81 & 84 & 0.44 \\
\hline Iliohypogastric & 42 & 46 & 41 & 0.28 \\
\hline Genitofemoral & 50 & 50 & 51 & 0.89 \\
\hline \multicolumn{5}{|l|}{ Neurectomy (\%) } \\
\hline Ilioinguinal & 25 & 22 & 29 & 0.16 \\
\hline Iliohypogastric & 12 & 15 & 10 & 0.17 \\
\hline Genitofemoral & 11 & 9 & 13 & 0.29 \\
\hline
\end{tabular}

* VAS = Visual Analogue Scale $;+A S A=$ Association of Anaesthesiologists classification; $\neq$ CRPS = Complex Regional Pain Syndrome 


\section{Primary outcome}

Pain steadily decreased over time in both groups in a similar fashion $(\mathrm{p}=0.471$; figure 3.2). Three years after the operation, $a>40$ point VAS score (moderate pain) was reported by just $3.7 \%$ of patients with a Progrip ${ }^{\text {tw }}$ and by $3.7 \%$ receiving a standard mesh. Severe pain (VAS $>70$ ) was not detected in any of the 274 patients.

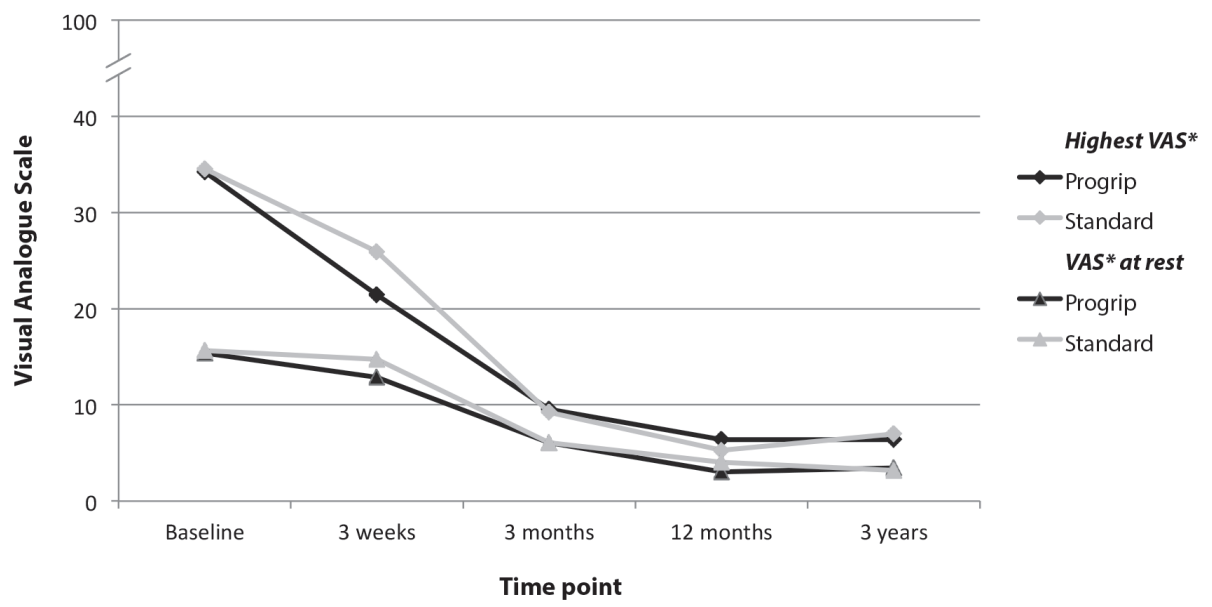

FIGURE 3.2:

Pain at rest and highest pain level in week preceding evaluation in patients receiving a Progrip ${ }^{\mathrm{TM}}$ or polypropylene mesh for primary inguinal hernia repair over time

*VAS = Visual Analogue Scale.

\section{Altered groin skin sensations}

Characteristics of altered groin skin sensation at various time points are described in table 3.2. At the one year evaluation, rates of neuropathic abnormalities including hypoesthesia, hyperesthesia and allodynia in the groin area were greatly attenuated compared to three weeks postoperatively. Three year following surgery, $2.2 \%$ of the Progrip $^{\text {TM }}$ patients and $3.7 \%$ of the standard mesh group still experienced hyperesthesia in the operated area. Presence of hyperesthesia was not correlated to a previous neurectomy (Spearman coefficient $-0.08, \mathrm{p}=0.42$ ). Hypoesthesia was frequently found (Progrip ${ }^{\mathrm{mm}}$ $12.2 \%$ vs. standard $19.3 \%$ ) and was also not related to a previous neurectomy (Spearman coefficient $0.17, \mathrm{p}=0.08)$. 
TABLE 3.2:

Neuropathic and nociceptive pain syndromes and sexual function 3 years after Progrip ${ }^{\mathrm{TM}}(P)$ or standard mesh (S) inguinal hernia repair

\begin{tabular}{|c|c|c|c|c|c|c|c|c|c|c|}
\hline & \multicolumn{2}{|c|}{$\begin{array}{l}\text { At baseline } \\
(n=363)\end{array}$} & \multicolumn{2}{|c|}{$\begin{array}{c}\text { After } 3 \\
\text { weeks } \\
(n=363)\end{array}$} & \multicolumn{2}{|c|}{$\begin{array}{l}\text { After } 3 \\
\text { months } \\
(n=363)\end{array}$} & \multicolumn{2}{|c|}{$\begin{array}{c}\text { After } 1 \text { year } \\
(n=335)\end{array}$} & \multicolumn{2}{|c|}{$\begin{array}{c}\text { After } 3 \text { year } \\
(\mathrm{n}=\mathbf{2 7 4})\end{array}$} \\
\hline & $\mathbf{P}$ & $\mathbf{s}$ & $\mathbf{P}$ & $\mathbf{s}$ & $\mathbf{P}$ & $\mathbf{S}$ & $\mathbf{P}$ & $\mathbf{S}$ & $\mathbf{P}$ & $\mathbf{s}$ \\
\hline \multicolumn{11}{|c|}{ Neuropathic characteristics (\%) } \\
\hline Hypoesthesia & 4.4 & 8.4 & 46.7 & 49.7 & \multicolumn{2}{|c|}{ NA } & 9.5 & 15.6 & 12.2 & 19.3 \\
\hline Hyperesthesia & 2.2 & 0.6 & 5.5 & 3.9 & \multicolumn{2}{|c|}{ NA } & 0 & 1.8 & 2.2 & 3.7 \\
\hline Allodynia & 0 & 0.6 & 1.1 & 0.6 & \multicolumn{2}{|c|}{ NA } & 0.6 & 0 & 0 & 0 \\
\hline Triggerpoint identifiable & \multicolumn{2}{|c|}{ NA } & \multicolumn{2}{|c|}{ NA } & \multicolumn{2}{|c|}{ NA } & 2.4 & 1.2 & 5.0 & 5.2 \\
\hline \multicolumn{11}{|c|}{ Nociceptive characteristics (\%) } \\
\hline Foreign body feeling & \multirow{3}{*}{\multicolumn{2}{|c|}{ NA }} & 27.4 & 29.3 & 34.6 & 32.1 & 8.3 & 12.0 & 13.6 & 22.3 \\
\hline Yes, painful & & & 4.9 & 7.2 & 3.3 & 5.0 & 0 & 1.2 & 1.4 & 3.0 \\
\hline Yes, not painful & & & 22.5 & 22.1 & 31.3 & 27.1 & 8.3 & 10.8 & 12.2 & 19.3 \\
\hline \multicolumn{11}{|l|}{ Sexual complaints (\%) } \\
\hline Impotence & 12.7 & 7.1 & 4.4 & 3.3 & \multicolumn{2}{|c|}{ NA } & 3.0 & 3.0 & 8.6 & 8.1 \\
\hline Painful erection & 1.8 & 0.6 & 0.5 & 1.1 & \multicolumn{2}{|c|}{ NA } & 1.2 & 0.6 & 0.7 & 0 \\
\hline Dysejaculation* & 1.8 & 1.8 & 0.5 & 2.2 & \multicolumn{2}{|c|}{ NA } & 1.2 & 0.6 & 0 & 0 \\
\hline
\end{tabular}

No group differences were observed for any of the variables;

$P=$ Progrip $^{\mathrm{TM}}$ mesh $; \mathrm{S}=$ standard sutured mesh;

$N A=$ Not Available;

${ }^{*}$ Dysejaculation = a painful ejaculation ${ }^{29}$.

A substantial portion of patients reported a foreign body feeling at one and three years postoperatively but group differences were not found. Interestingly, these patients were not suffering from pain. A nociceptive pain syndrome such as periostitis was only present in $0.7 \%$ of Progrip $^{\text {Ti }}$ group compared to $0 \%$ in patients with conventional mesh. No patients experienced iliopectineal bursitis, adductor tendinitis or referred lumbosacral pain at the three year follow-up. A painful erection or dysejaculation was not reported. 


\section{Type of hernia and three year recurrence rate}

The number of direct hernias was 36 in the Progrip ${ }^{\mathrm{mm}}$ group and 35 in the standard group $(\mathrm{p}=0.86)$. The proportion of indirect hernia was the same in both treatment groups (Progrip $^{\mathrm{mm}}, \mathrm{n}=62$ versus standard, 53, $\mathrm{p}=0.50$ ). A combination of an indirect and a direct hernia was found in 28 Progrip ${ }^{\text {m" }}$ patients and 32 standard patients ( $\mathrm{p}=0.38$ ). In 28 patients the data on type of hernias were missing.

At the first year follow up visit, 14 hernia recurrences were found (Progrip ${ }^{\mathrm{mw}}, \mathrm{n}=10$ versus standard, $\mathrm{n}=4 ; \mathrm{p}=0.10$ ). Nine additional recurrences occurred after the one-year follow-up time point (Progrip ${ }^{\text {two }}, \mathrm{n}=6$ versus standard, $\mathrm{n}=3$ ) resulting in 23 recurrences at the three year follow-up period (Progrip ${ }^{\mathrm{m}}, \mathrm{n}=16$ vs. standard, $\mathrm{n}=7 ; \mathrm{p}=0.05$ ).

Of the 16 Progrip $^{\text {mis }}$ patients who had a recurrence, four had an indirect hernia, six had a direct hernia and five patients had a combination of both. In one patient intraoperative data on the type of hernia were missing. In the standard group with a recurrence, one patient had an indirect hernia, four patients had a direct hernia and remaining two had both types.

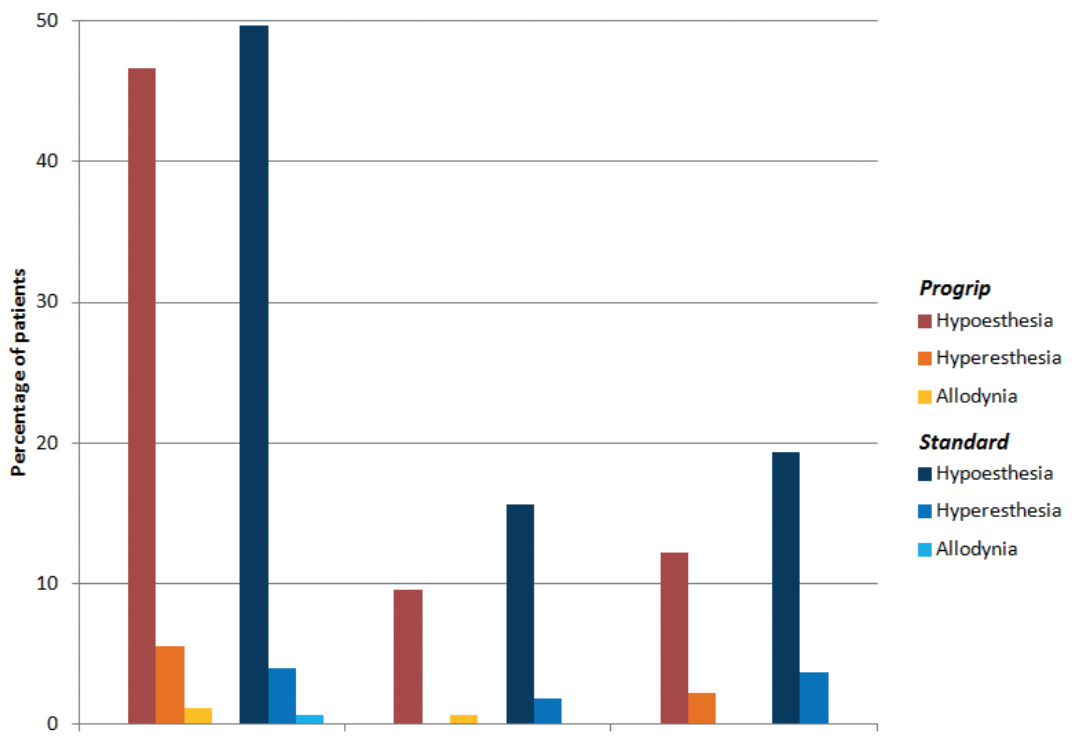

FIGURE 3.3:

Sensory disturbances following inguinal hernia repair after Progrip ${ }^{\mathrm{TM}}$ or standard mesh. Differences between groups at various time points were not statistically significant. 


\section{DISCUSSION}

This is the first randomized trial comparing two types of inguinal hernia meshes after three years, a minimal follow-up period for determining long-term-outcome for pain as advised by a recent European Hernia Society recommendation ${ }^{8}$. The aim was to report on intensity and characteristics of long-term pain and recurrence rates following an open hernia repair. In the long term, pain levels appeared very low in both groups (highest VAS of 7 on a 0-100 scale). However, recurrence rates were more than twice as high in the Progrip ${ }^{\mathrm{ma}}$ mesh compared to the standard sutured mesh. The present findings may aid surgeons in counselling hernia patients on the two most important end points in hernia surgery, pain characteristics and recurrence rates.

It was previously doubtful whether self-gripping meshes are superior regarding postherniorrhaphy pain when compared to conventional polypropylene meshes. Earlier studies on self-gripping meshes demonstrated conflicting results as some claimed that self-gripping meshes cause less pain ${ }^{3,5}$ whereas the opposite was stated by others one month following surgery ${ }^{2}$. Systematic reviews and meta-analyses did not demonstrate any statistically significant differences although a large overlap of included studies was found ${ }^{4,15-18}$. The present data demonstrate that the highest pain level at three years was a 6.4 VAS in the Progrip ${ }^{\text {tx }}$ and a 7.0 VAS in the polypropylene group (on a 100-point scale). These levels are so low that any potential clinical relevance is absent. It is concluded that a Progrip $^{\mathrm{Tm}}$ self-gripping mesh confers similar pain levels following inguinal hernia repair on the long term when compared to standard mesh.

The present long-term study also found that characteristics of pain do not differ between the Progrip ${ }^{\mathrm{rm}}$ and the conventional mesh group at any of the time points during the three year follow-up. Others have previously investigated impaired inguinal sensibility and found no significant disturbances at a 12-month follow-up ${ }^{2}$. In the present trial however, both hypoesthesia and hyperesthesia show a progressive decrease up to one year following inguinal hernia repair but, surprisingly, an increase towards the three years evaluation. A slight correlation (Spearman coefficient 0.17) was found between a prophylactic neurectomy during hernia repair and hypoesthesia. Although neuropathic abnormalities did not significantly differ between the two groups, a trend towards more hyperesthesia in standard mesh (2.2 vs 3.7\%, p=0.08) was demonstrated. In 2008, Aasvang et al. questioned whether the underlying pathophysiological mechanisms 
of altered groin skin sensation could be related to either a direct intraoperative nerve injury or progressive nerve injury due to an ongoing inflammatory response to mesh ${ }^{19}$. Considering an increased sensory disturbance rate after three years in the present series, mechanisms related to an inflammatory response associated with the inserted mesh are plausible.

A foreign body feeling was present three months postoperatively in one of three patients in both groups. However, rates declined during the first year but were again higher after three years. Previous studies suggested that up to half of the patients with a sutured mesh-based hernia repair report a foreign body feeling in the groin ${ }^{20-22}$. Trials comparing a standard and a lightweight mesh have demonstrated a significantly lower rate of foreign body sensation in the latter group ${ }^{3,20}$. The present study also found a trend $(\mathrm{p}=0.06)$ for a lower rate of foreign body feeling three years after a Progrip ${ }^{\mathrm{TN}}$. In contrast, more than one in five patients having a sutured mesh reported this phenomenon at three years. The reason for this phenomenon is unclear but is possibly related to more intense fibrosis and adhesions to surrounding structures. Whether this foreign body feeling is also directly related to mesh shrinkage or meshoma formation needs to be clarified.

As more recurrences were demonstrated at 3 years than at 1 year follow-up, the time course of recurrence after mesh repair is yet to be fully defined. In the present trial, recurrences were significantly more prevalent in the self-gripping mesh after three years of follow-up. Due to its semi-resorbable, lighter and self-gripping characteristics, Progrip ${ }^{\mathrm{mm}}$ was postulated to have a relative intrinsic weakness when compared to a conventional polypropylene mesh ${ }^{3,23}$. As a consequence, Progrip ${ }^{\mathrm{Tm}}$ mesh may induce less formation of fibrosis and may potentially lead to higher recurrence rates. However, previous prospective studies and meta-analyses comparing Progrip ${ }^{\mathrm{Tm}}$ and conventional meshes studies failed to confirm different recurrences rates ${ }^{2,4,5,15,16,18,24-28}$. Number of recurrences may also be related to the absence of suture fixation in the self-gripping mesh. However, one retrospective database study evaluating 2,095 patients did not observe different recurrence rates between groups with and without additional fixation of self-gripping meshes after one year ${ }^{24}$. Prospective studies beyond two years are not available. The oneyear results of the present study also failed to demonstrate any differences in recurrence rates. A three year follow-up period seems a requisite for demonstrating reliable numbers of recurrences after inguinal hernia repair as demonstrated in the present trial. 
It may seem that the overall 5\% recurrence rate in the current study is relatively high. As stated before, $76 \%$ of the procedures were done by junior trainees under supervision, $17 \%$ were done by a senior trainee alone, and just $6 \%$ by a trained surgeon ${ }^{9}$. It is believed that this $5 \%$ recurrence rate and a relatively high percentage of residents doing the hernia repair likely reflects the situation in real life around the globe. Surely, the vast majority of hernia repairs are performed in general hospitals rather than by dedicated hernia surgeons in centers of expertise. Although previous studies demonstrated higher recurrence rates after direct inguinal hernias ${ }^{29,30}$, we did not find such results in our study, as demonstrated by the equal contribution of (in)direct hernias in both treatment groups.

In conclusion, three years after open herniorrhaphy comparing a light Progrip ${ }^{\text {Tw }}$ and a standard polypropylene mesh, pain levels are comparably low in both groups but hernia recurrence rates are higher in the Progrip ${ }^{\mathrm{Tm}}$ group. Signs of altered groin skin sensation increased over time in both groups. However, a foreign body feeling was more prevalent in the standard sutured mesh after three years. Studies with longer follow-up will determine the clinical relevance of these findings.

\section{Other information}

Sources of funding were a restricted grant from Covidien. No other supportive sources are declared. 


\section{REFERENCE LIST}

1. Usher FC, Cogan JE, Lowry TI. A new technique for the repair of inguinal and incisional hernias. Arch Surg 1960;81:847-54.

2. Jorgensen LN, Sommer T, Assaadzadeh S, et al. Randomized clinical trial of self-gripping mesh versus sutured mesh for Lichtenstein hernia repair. Br J Surg 2013;100(4):474-81.

3. Weyhe D, Belyaev O, Muller C, et al. Improving outcomes in hernia repair by the use of light meshes--a comparison of different implant constructions based on a critical appraisal of the literature. World J Surg 2007;31(1):234-44.

4. Sanders DL, Waydia S. A systematic review of randomized control trials assessing mesh fixation in open inguinal hernia repair. Hernia 2014;18(2):165-76.

5. Kingsnorth A, Gingell-Littlejohn M, Nienhuijs S, et al. Randomized controlled multicenter international clinical trial of self-gripping Parietex ProGrip polyester mesh versus lightweight polypropylene mesh in open inguinal hernia repair: interim results at 3 months. Hernia 2012;16(3):287-94.

6. Grant AM, Scott NW, O’Dwyer PJ. Five-year follow-up of a randomized trial to assess pain and numbness after laparoscopic or open repair of groin hernia. Br J Surg 2004;91(12):1570-4.

7. Nienhuijs S, Staal E, Strobbe L, et al. Chronic pain after mesh repair of inguinal hernia: a systematic review. Am J Surg 2007;194(3):394-400.

8. Simons MP, Aufenacker T, Bay-Nielsen M, et al. European Hernia Society guidelines on the treatment of inguinal hernia in adult patients. Hernia 2009;13(4):343-403.

9. Verhagen T, Zwaans WA, Loos MJ, et al. Randomized clinical trial comparing self-gripping mesh with a standard polypropylene mesh for open inguinal hernia repair. Br J Surg 2016;103(7):812-8.

10. Moher D, Hopewell S, Schulz KF, et al. CONSORT 2010 explanation and elaboration: updated guidelines for reporting parallel group randomized trials. BMJ 2010;340:c869.

11. Baron R. Mechanisms of disease: neuropathic pain--a clinical perspective. Nat Clin Pract Neurol 2006;2(2):95-106.

12. van Assen T, de Jager-Kievit JW, Scheltinga MR, et al. Chronic abdominal wall pain misdiagnosed as functional abdominal pain. J Am Board Fam Med 2013;26(6):738-44.

13. Smeds S, Nienhuijs S, Kullman E, et al. Identification and management of the ilio-inguinal and ilio-hypogastric nerves in open inguinal hernia repair: benefits of self-gripping mesh. Hernia 2016;20(1):33-41. 
14. Wijsmuller AR, van Veen RN, Bosch JL, et al. Nerve management during open hernia repair. Br J Surg 2007;94(1):17-22.

15. Zhang C, Li F, Zhang H, et al. Self-gripping versus sutured mesh for inguinal hernia repair: a systematic review and meta-analysis of current literature. J Surg Res 2013;185(2):653-60.

16. Pandanaboyana S, Mittapalli D, Rao A, et al. Meta-analysis of self-gripping mesh (Progrip) versus sutured mesh in open inguinal hernia repair. Surgeon 2014;12(2):87-93.

17. Fang Z, Zhou J, Ren F, et al. Self-gripping mesh versus sutured mesh in open inguinal hernia repair: system review and meta-analysis. Am J Surg 2014;207(5):773-81.

18. Li J, Ji Z, Li Y. The comparison of self-gripping mesh and sutured mesh in open inguinal hernia repair: the results of meta-analysis. Ann Surg 2014;259(6):1080-5.

19. Aasvang EK, Brandsborg B, Christensen B, et al. Neurophysiological characterization of postherniotomy pain. Pain 2008;137(1):173-81.

20. Post S, Weiss B, Willer M, et al. Randomized clinical trial of lightweight composite mesh for Lichtenstein inguinal hernia repair. Br J Surg 2004;91(1):44-8.

21. Zwaans WA, Perquin CW, Loos MJ, et al. Mesh Removal and Selective Neurectomy for Persistent Groin Pain Following Lichtenstein Repair. World J Surg 2017;41(3):701-12.

22. Loos MJ, Roumen RM, Scheltinga MR. Classifying post-herniorrhaphy pain syndromes following elective inguinal hernia repair. World J Surg 2007;31(9):1760-5; discussion 66-7.

23. Burgmans JP, Voorbrood CE, Simmermacher RK, et al. Long-term Results of a Randomized Double-blinded Prospective Trial of a Lightweight (Ultrapro) Versus a Heavyweight Mesh (Prolene) in Laparoscopic Total Extraperitoneal Inguinal Hernia Repair (TULP-trial). Ann Surg 2016;263(5):862-6.

24. Kohler G, Lechner M, Mayer F, et al. Self-Gripping Meshes for Lichtenstein Repair. Do We Need Additional Suture Fixation? World J Surg 2016;40(2):298-308.

25. Quyn AJ, Weatherhead KM, Daniel T. Chronic pain after open inguinal hernia surgery: suture fixation versus self-adhesive mesh repair. Langenbecks Arch Surg 2012;397(8):1215-8.

26. Chastan P. Tension-free open hernia repair using an innovative self-gripping semi-resorbable mesh. Hernia 2009;13(2):137-42.

27. Anadol AZ, Akin M, Kurukahvecioglu O, et al. A prospective comparative study of the efficacy of conventional Lichtenstein versus self-adhesive mesh repair for inguinal hernia. Surg Today 2011;41(11):1498-503.

28. Pierides G, Scheinin T, Remes V, et al. Randomized comparison of self-fixating and sutured mesh in open inguinal hernia repair. Br J Surg 2012;99(5):630-6. 
29. Kald A, Nilsson E, Anderberg B, et al. Reoperation as surrogate endpoint in hernia surgery. A three year follow-up of 1565 herniorrhaphies. Eur J Surg 1998;164(1):45-50.

30. Burcharth J, Pommergaard HC, Bisgaard T, et al. Patient-related risk factors for recurrence after inguinal hernia repair: a systematic review and meta-analysis of observational studies. Surg Innov 2015;22(3):303-17.

$62 \mid$ Strategies for chronic inguinal pain 



\title{
CHAPTER 4
}

Mesh removal and selective neurectomy for persistent groin pain following Lichtenstein repair

\author{
W.A.R. Zwaans
}

C.W. Perquin

M.J.A. Loos

R.M.H. Roumen

M.R.M. Scheltinga

World J Surg 2017; 41(3): 701-712 


\section{ABSTRACT}

Background: Some patients with persistent inguinodynia following a Lichtenstein hernia repair fail all non-surgical treatments. Characteristics of mesh-related pain are not well described whereas a meshectomy is controversial. Aims were to define mesh-related pain symptoms, to investigate long-term effects of a meshectomy and to provide recommendations on meshectomy.

Methods: Consecutive patients undergoing open meshectomy with/without selective neurectomy for chronic inguinodynia following Lichtenstein repair were analyzed including a follow-up questionnaire. Outcome measures were complications, satisfaction (excellent, good, moderate, poor) and hernia recurrence rate. Recommendations for meshectomy are proposed based on a literature review.

Results: Seventy-four patients (67 males, median age 56 years) underwent mesh removal (exclusively mesh, 26\%; combined with tailored neurectomy, $74 \%$ ) between June 2006 and March 2015 in a single centre. Complications were intraoperatively recognized small bowel injury $(n=1)$ and testicular atrophy $(n=2)$. A 64\% excellent/ good long-term result was attained (median 18 months). Success rates of a meshectomy (63\%) or combined with a neurectomy (64\%) were similar. Five hernia recurrences occurred during follow-up (7\%). A patient with a pure mesh-related groin pain characteristically reports a 'foreign body feeling. Pain intensifies during hip flexion (car driving) and is attenuated following hip extension or supine position. Palpation is painful along the inguinal ligament whereas neuropathic characteristics (hyperpathic skin, trigger points) are lacking.

Conclusions: Mesh removal either or not combined with tailored neurectomy is beneficial in two of three patients with characteristics of mesh-related inguinodynia following Lichtenstein hernia repair who are refractory to alternative pain treatments. 


\section{INTRODUCTION}

The gold standard for inguinal hernia repair is a mesh-based technique, either via an open or an endoscopic approach ${ }^{1}$. The introduction of prosthetic material has significantly reduced hernia recurrence rates. Therefore, research nowadays has shifted towards studying specifics of chronic postoperative groin pain, defined as pain beyond a three months postoperative period ${ }^{2}$. Approximately one-third of the patients suffering from persistent postherniorrhaphy inguinodynia experience limitations in daily leisure activities $^{1}$. Consequently, an adverse impact on quality of life is reported ${ }^{3}$. Laparoscopic inguinal hernia repair has reduced postoperative pain rates ${ }^{1,4,5}$. However, still a $11 \%$ pooled incidence of postherniorrhaphy inguinodynia was observed in a meta-analysis of 34 studies $^{1}$.

The results of initially available therapies for inguinodynia after standard herniorrhaphy were disappointing. Experimental surgical studies in the last decade, however, showed better results in reducing pain in patients that were previously unresponsive to conservative pain management ${ }^{6-8}$. As the pain often is deemed of neuropathic (nerverelated) origin, surgical treatment including a neurectomy of the ilioinguinal nerve, occasionally in combination with iliohypogastric or genitofemoral nerve resection, is frequently considered.

Unfortunately, a subgroup of inguinodynia patients after open hernia repair does not respond to a neurectomy. These patients may have a predominantly nociceptive or inflammatory type of inguinodynia that is probably due to presence of the mesh. However, precise descriptions of clinical characteristics of a typical mesh-related pain are currently lacking. Mesh removal is sometimes advised by experts, either as a means to facilitate inguinal neurectomy or if the mesh itself is implicated on subjective grounds as a major source of pain ${ }^{6,9,10}$. Mesh removal as a first line surgical treatment is also controversial due to the scarcity of studies and the fear of hernia recurrences. Clear guidelines or instructions for clinical practise regarding the removal of meshes are currently not available.

The present study investigates the effects of mesh removal (with or without selective neurectomy) in a large homogenous group of patients with pain following a previous Lichtenstein operation. It is hypothesized that mesh removal with or without selective neurectomy is effective in reducing pain in most patients, whereas number of complications 
and recurrences are limited. An additional aim is to propose recommendations for meshectomy, based on available literature.

\section{METHODS}

\section{Study Characteristics}

This retrospective study was performed in the Department of General Surgery of Máxima Medical Centre (MMC), the Netherlands. Surgeons of MMC have a special interest in patients with chronic abdominal wall pain and groin pain. Outpatient department activities are performed in a centre of expertise (SolviMáx) that is exclusively dedicated to evaluation of these patients. An increasing number of such patients, the majority referred by other hospitals, are being evaluated (2014: $n=840$ ) at SolviMáx. Approximately one third of referrals are diagnosed with 'postherniorrhaphy inguinodynia'. Patients undergoing open surgery for inguinodynia after a standard hernia repair according to Lichtenstein, executed between January $1^{\text {st }}, 2000$ and March 31 $1^{\text {st }}, 2015$ were identified via an exclusive SolviMáx operative code that is assigned to each inguinal neurectomy and/ or meshectomy. Subsequently, data of possible eligible patients were entered in a separate database for further analysis. The study was conducted according to the declaration of Helsinki. The local MMC ethics committee judged that evaluation of the study protocol was not required since this effort was considered a normal evaluation of patient outcome measurement and auditing of own results.

\section{Eligibility criteria participants}

Inclusion criteria were chronic ( $\geq 3$ months) inguinodynia after a Lichtenstein repair for primary inguinal hernia, age $\geq 18$, mesh removal for predefined mesh-related pain symptoms (figure 4.1) and presence of operative report. All patients had previously undergone a variety of conservative measures including medication, injection therapy and additional pain treatments that were performed in the referring institutions with limited success. Patients with mesh removal for pain following femoral hernia repair or other primary hernia repair techniques were excluded, as were patients in whom a small rim of mesh was removed for pragmatic perioperative considerations (improving visualisation). Furthermore, patients with a malignancy, cognitive impairment, meralgia paresthetica and (low grade) groin infections were also excluded. 


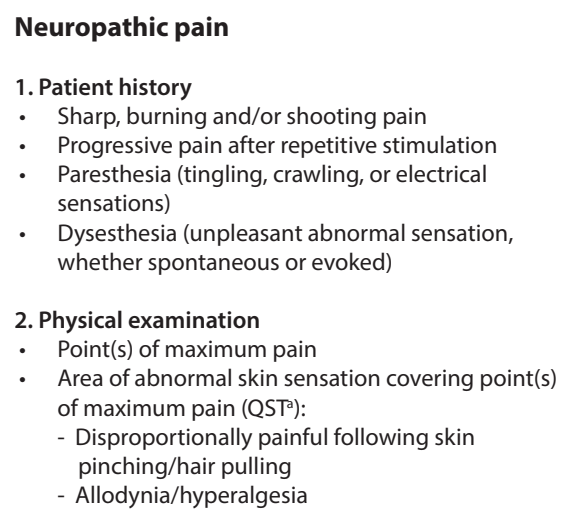

1. Patient history

- Sharp, burning and/or shooting pain

- Progressive pain after repetitive stimulation

- Paresthesia (tingling, crawling, or electrical sensations)

- Dysesthesia (unpleasant abnormal sensation, whether spontaneous or evoked)

2. Physical examination

- Point(s) of maximum pain

- Area of abnormal skin sensation covering point(s) of maximum pain (QST):

- Disproportionally painful following skin pinching/hair pulling

- Allodynia/hyperalgesia

\section{Mesh-related pain}

1. Patient history

- 'Foreign body feeling'/ sensation of tightness in groin area

- Pain aggravation during car driving, crossing legs, exercise and/or sexual activity

- Pain relief by hip extension or supine position

2. Physical examination

- Painful deep palpation along the inguinal ligament $\geq 5 \mathrm{~cm}$

- Lack of characteristics of neuropathic pain

- No points of maximum pain but diffuse

- Lump/mesh(oma) palpable (in non-obese patients)

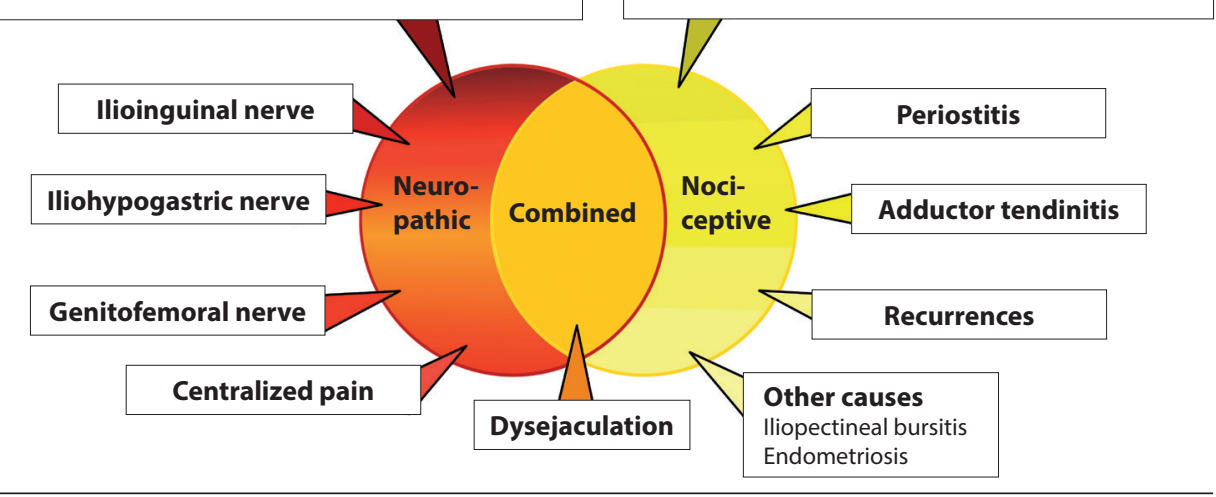

FIGURE 4.1:

Types of postherniorrhaphy pain and characteristics of "pure" mesh-related en neuropathic pain.

Characteristics of neuropathic pain were previously described ${ }^{31,32} \cdot{ }^{a}$ QST $=$ Quantitative Sensory Testing

\section{Data accrual}

Electronic patient files were used for extracting specifics including age, gender, height, weight, body mass index, date of Lichtenstein repair, history of preperitoneal mesh placement, radiologic imaging, date and side of mesh removal, operation time, type of anaesthesia, surgeon, formation of meshoma (macroscopic impression: defined as folding and wrinkling of the inserted mesh, varying from a mass-like density to a more subtle wrinkling or fibrosis ${ }^{11,12}$ ), intraoperative complications, histopathological confirmation of neuroma, postoperative complication, patient satisfaction, Numerical Rating Scale (NRS) score at baseline and time of follow-up, date of being pain free and recurrences.

Outpatient follow-up was continued by the operating surgeon until a satisfactory result was attained, or when additional surgical consultation was not considered meaningful. A 
final follow-up control was performed by the first author using a standardized telephone interview in the period of February, 2014 to July, 2015. This interview included questions on present levels of pain, satisfaction, long-term complications, presence of current bulging in proximity to or underneath the scar and other typical complaints of a recurrent hernia. All data were anonymized.

\section{Review strategy on studies containing mesh removal for inguinodynia}

Available literature on mesh removal for mesh-related pain was obtained by a full electronic search strategy using the electronic databases PubMed and Embase. The following syntax was used to select relevant papers: “(((()((“Surgical Mesh”[Mesh])) OR (“Device Removal”[Mesh])) OR (mesh removal))) AND (((((((“Hernia, Inguinal”[Mesh])) OR (“Herniorrhaphy”[Mesh])) OR (inguinal hernia)) OR (inguinal herniorrhaphy)) OR (inguinal hernioplasty)) OR (inguinal hernia repair))) AND $(((($ "Pain, Postoperative”[Mesh])) OR (postoperative pain)) OR (“Pain”[Mesh]))) AND $(((($ (“Pain Management”[Mesh] $))$ OR (pain management)) OR (“Reoperation”[Mesh])) OR (reoperation))". The following terms free text words were used in Embase to search for relevant papers: 'device removal' AND 'inguinal hernia' AND 'postoperative pain' OR 'chronic pain'. The search strategy was performed up to September $8^{\text {th }}, 2015$. Potential papers were selected on the abstract contents, and reference lists were screened to ensure no relevant papers were missed.

\section{Eligibility criteria studies for review}

Case series, cohort studies, case-control studies and randomized controlled trials were all considered if the main aim was to investigate the results of a meshectomy, whether or not combined with a tailored or triple neurectomy. Studies were not eligible when patients with just neuropathic pain syndromes were evaluated, or when patients suffering from nociceptive pain were excluded as defined by the authors. The full, original report of the study had to be available. No language restrictions were applied.

\section{Data accrual of included studies}

Data that were extracted from papers included total number of patients, number of patients who underwent mesh removal, delay from primary repair to meshectomy, age, study design, anterior or endoscopic approach for meshectomy, percentage mesh removals 
combined with a neurectomy, type of primary inguinal hernia repair, preoperative and postoperative pain status, definition of success, percentage of successfully operated patients following own definition, hernia recurrence rate and follow-up. Missing data that were considered crucial were tried to retrieve from the corresponding author by an electronic approach. If information in the paper was unclear, authors were also approached for additional data.

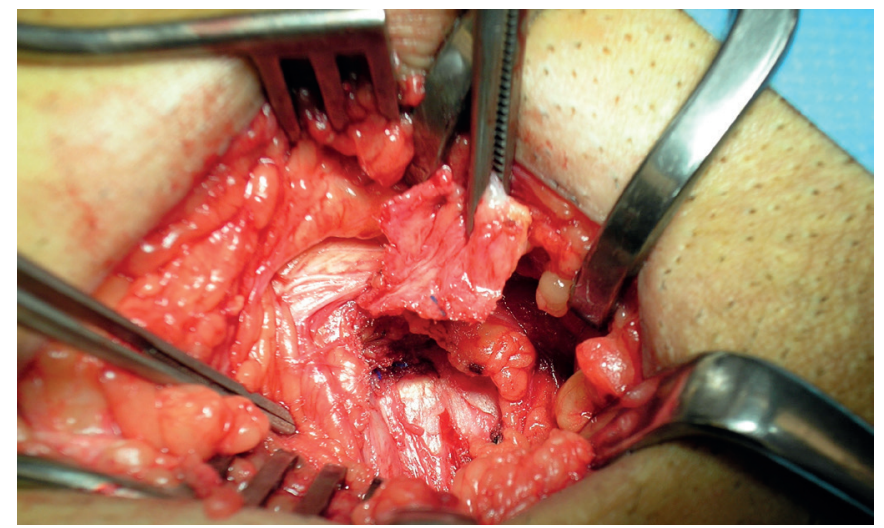

FIGURE 4.2:

Intraoperative view of mesh removal (piecemeal)

\section{Specifics of open mesh removal}

Patients were counselled on the pros and cons of mesh removal. Potential complications that were discussed included testicular atrophy, hematomas, wound infection and recurrent hernias. All patients consented verbally and in writing to the specifics of the operation. The operation was performed as open surgery. The aponeurosis of the external abdominal muscle was exposed using the previous groin incision followed by partial or complete mesh removal including residual suture material (figure 4.2). All operations were performed by two surgeons (RR, MS). The decision to perform an additional neurectomy depended on patient history, physical examination and intraoperative findings. If an inguinal nerve was deemed entrapped, or if proper execution of the mesh removal was likely complicated by its presence, the nerve was sacrificed and histopathologically examined. Once the mesh was removed, a local tissue repair technique was used to close the (predominantly fibrotic) layers of the abdominal wall. Using this technique, tissue 
of the conjoined tendon and the fascia of the external oblique muscle is fixed to the innermost portion of the inguinal ligament. Some $15-20 \mathrm{ml}$ of $0.25 \%$ bupivacaine was used to infiltrate the wound. Patients were usually admitted for one night.

\section{Outcomes}

Primary outcome measure was patient satisfaction at the final follow-up. A four point scale was used (1. excellent - I am pain free, 2. good - I am almost pain free, 3. moderate - although there is some pain reduction, I am still frequently bothered by pain complaints, 4. poor - the operation had no effect and the pain is virtually the same or worse $)^{6}$. The operation was termed successful if patient satisfaction was excellent or good. Current levels of pain were also obtained during final follow-up using a NRS pain scale and compared with preoperative pain scores obtained from the electronic patient files, if present. The NRS is a simple one-dimensional test that instructs individuals to score pain on a 0 (no pain) to 10 (unbearable pain) scale.

Secondary outcomes were short-term ( $\leq 3$ months) complications including hematoma, infection, wound dehiscence, testicular atrophy, urinary tract infection, ileus, thrombosis, pulmonary or cardiac complications, and long-term complications including hernia recurrence. A hernia was deemed a recurrence on the basis of a reported sudden or gradual development of a palpable swelling underneath or in close proximity to the inguinal scar.

For the purpose of generating evidence-based recommendations for mesh removal, recapitulation of the available data on meshectomies in current literature was performed. Outcome was the success rate according to the definition of the concerning paper. Baseline characteristics of patients were collected from the original papers.

\section{Data analysis}

Data were analyzed using SPSS version 19.0 software (SPSS Inc., Chicago, IL, US) and were expressed as means ( \pm standard deviation), medians (range) or percentages (n) as appropriate. The primary outcome measure and complication rates were calculated using descriptive statistics. Recurrences were expressed as percentages. All operations were considered independently allowing determination of the effect of mesh removal. When patients underwent bilateral groin surgery, each side was analyzed separately. The correlation between outcome and follow-up was calculated using bivariate logistic regression analysis. P-value $<0.05$ was considered statistically significant. 


\section{RESULTS}

\section{Demographics and operative specifics}

A total of 496 operations for inguinal hernia repair related pain of any type were performed between June 30 $0^{\text {th }}, 2006$ and March 31 st 2015 in SolviMáx (figure 4.3). No meshes were removed before the starting point of the study. Seventy-nine operations met study inclusion criteria. Five patients were lost to follow-up leading to a 74 patient study population. Mesh removal combined with a selective neurectomy was performed in 55 patients, whereas 19 underwent a meshectomy only. In nine patients (12\%) mesh removal was performed as secondary remedial surgery, due to persisting pain following a neurectomy only. Five of these nine patients had an excellent result following mesh removal, a sixth patient experienced moderate pain relief and the remaining three patients did not benefit from the meshectomy. Baseline characteristics and specifics of surgery are summarized in table 4.1. Mean age was 56 years and most patients were male $(91 \%, n=67)$. Radiologic imaging preceding meshectomy was performed in the minority of patients, ultrasonography was the most frequently performed diagnostic modality (50\%). Median time from primary Lichtenstein repair to meshectomy was 35 months. Mean operation time was 66 minutes. An inguinal neurectomy was often required (74\%). A meshoma and a histopathologically confirmed neuroma were removed in $31 \%$ and $12 \%$ of the patients, respectively.

\section{Primary outcome}

At the median 18 months follow-up, results were excellent (50\%) or good (14\%), leading to a $64 \%$ successful outcome (figure 4.4). Conversely, a moderate (14\%) or poor $(22 \%)$ outcome was reported in the remaining one thirds $(36 \%)$. Pain after surgery was considered worse in five patients (7\%). Seventy percent $(n=52)$ did not regret surgery and would undergo mesh removal again knowing their present outcome. The remaining $30 \%$ of patients would not undergo surgery again. Median NRS score before mesh removal was 7.5 (interquartile range 6.5-8.0). Pain intensity dropped to 3.5 after the meshectomy (interquartile range 0.3-7.0, p<0.001). Successful outcome in patients with just mesh removal $(n=19)$ or following a combined mesh removal /selective neurectomy $(n=55)$ were similar (63\% and 64\%, respectively). No correlation was found between outcome and follow-up (odds ratio 0.99, 95\%CI 0.97-1.01). 


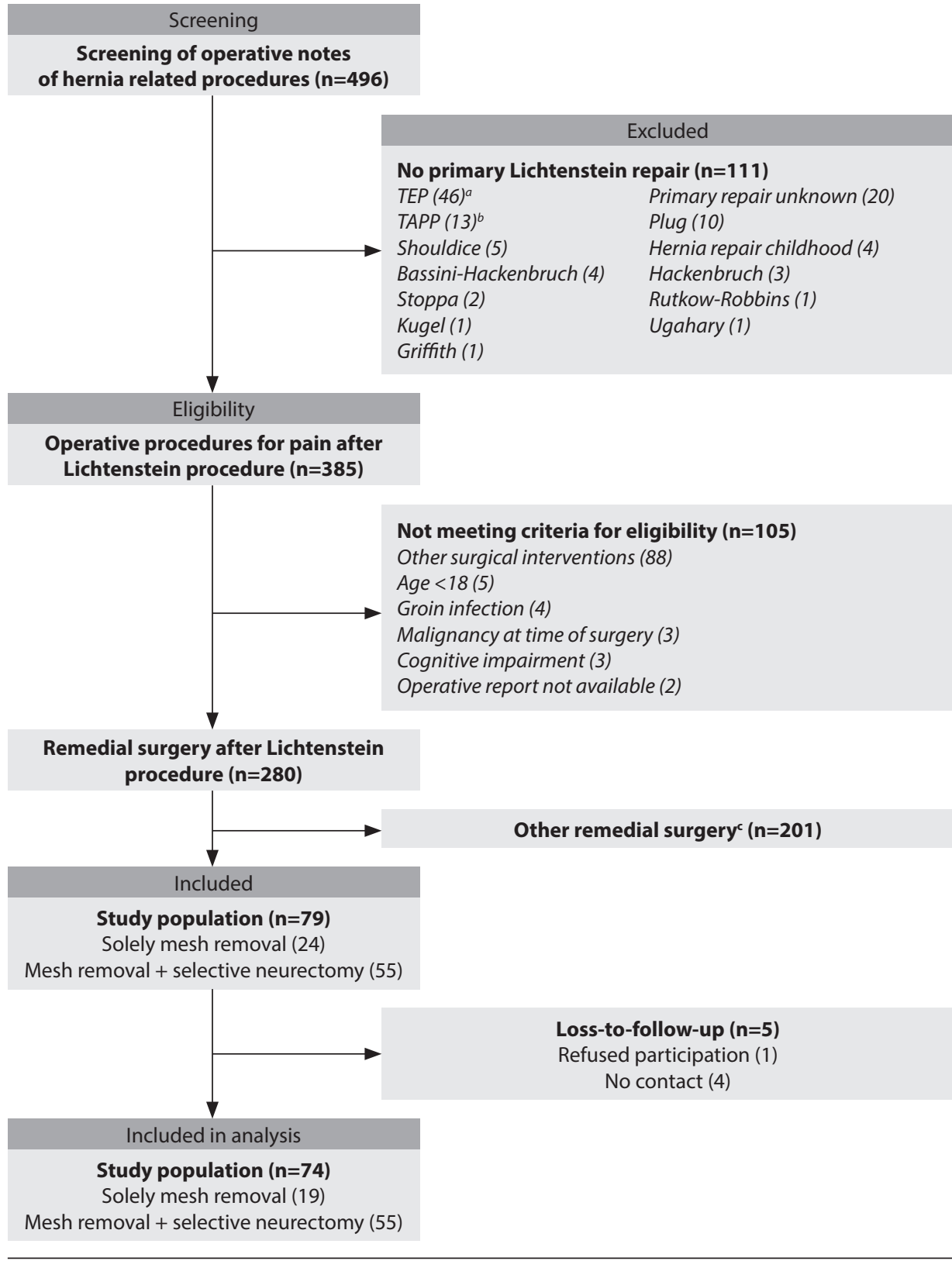

FIGURE 4.3:

Flowchart of selection of patients.

${ }^{a}$ TEP = Totally extra-peritoneal repair ${ }^{b}$ TAPP = Transabdominal preperitoneal repair; ${ }^{c}$ Tailored or a triple neurectomy. In some of these patients a small rim of the mesh was removed during the neurectomy for pragmatic considerations. This procedure was not considered a meshectomy. 


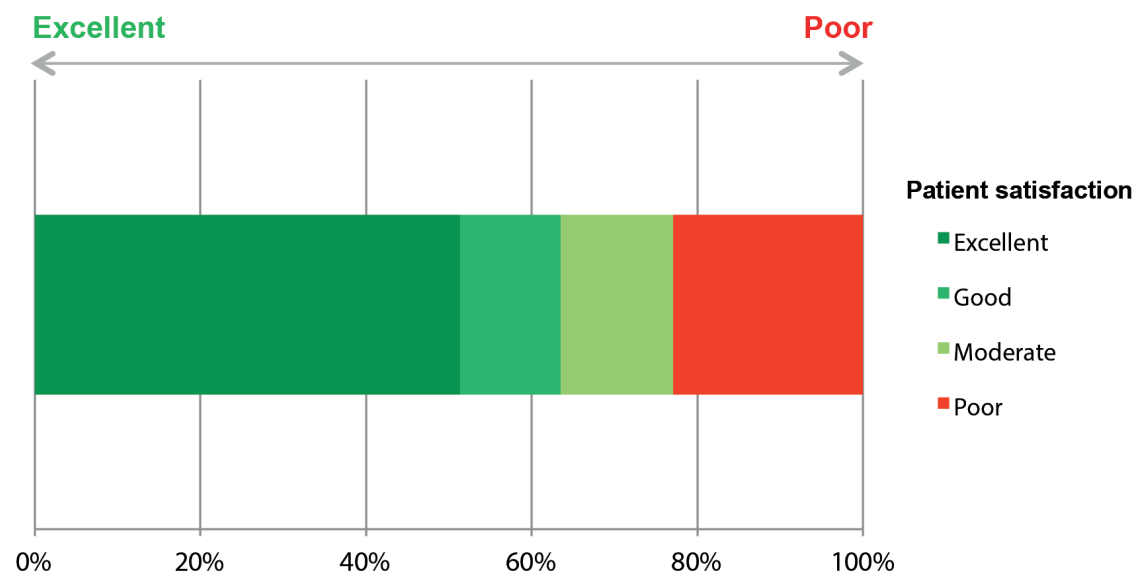

FIGURE 4.4:

Percentage of patients in relation to satisfaction level after mesh removal for postherniorrhaphy inguinodynia

\section{Complications after mesh removal}

Major complications (table 4.1) included a $10 \mathrm{~cm}$ resection of small bowel due to mesh penetration $(n=1)$. This patient recovered uneventful. Testicular atrophy due to damaged testicular vessels was observed in two patients. Ileus, thrombosis, pulmonary or cardiac events did not occur. Minor complications were hematomas ( $n=4$, operative drainage $n=1$, conservative treatment $n=3$ ) and superficial wound infection requiring successful drainage and antibiotic treatment $(n=2)$.

Five patients (7\%) developed a recurrent hernia. In four of these, a local tissue repair technique was used during the mesh removal procedure. These recurrences occurred after 3,11, 13 and 16 months. Three became symptomatic and were successfully treated using a preperitoneal mesh. The fourth recurrence was accidentally found on a routine MRI and was asymptomatic leading to a wait-and-see approach.

A total of 23 patients had, prior to the Lichtenstein mesh removal, also undergone a laparoscopic mesh implantation in their referral centre for presumed recurrent hernia or for pain (table 4.1). No preperitoneal meshes were removed in the present study, but at the 18 months final evaluation one recurrence (4\%) was observed in this subgroup, 8 months after meshectomy. 
TABLE 4.1:

Baseline characteristics and surgical specifics of patients undergoing mesh removal for postherniorrhaphy inguinodynia

\section{Patient characteristics at the time of surgery and intervention data $(n=74)$}

\begin{tabular}{|c|c|}
\hline Age (years) & $56 \pm 11$ \\
\hline Sex ratio (male/female) & $67: 7$ \\
\hline Body Mass Index $\left(\mathrm{kg} / \mathrm{m}^{2}\right)$ & $26.2[17.3-40.0]$ \\
\hline \multicolumn{2}{|l|}{ Side of hernia, $\mathrm{n}(\%)$} \\
\hline Left & $33(45)$ \\
\hline Right & $41(55)$ \\
\hline \multicolumn{2}{|l|}{ Radiologic examination preceding mesh removal, $\mathrm{n}(\%)$} \\
\hline Ultrasonography & $37(50)$ \\
\hline Computed tomography (CT) & $14(19)$ \\
\hline Magnetic resonance imaging (MRI) & $10(14)$ \\
\hline History of preperitoneal mesh placement, $\mathrm{n}(\%)$ & $23(31)$ \\
\hline Follow-up (months) & 18 [3-83] \\
\hline Time to reoperation / delay (months) & 35 [4-198] \\
\hline Operation time (minutes) & $66 \pm 24$ \\
\hline \multicolumn{2}{|l|}{ Anaesthesia, $\mathrm{n}(\%)$} \\
\hline General & $28(38)$ \\
\hline Spinal & $46(62)$ \\
\hline Neurectomy, n (\%) & $55(74)$ \\
\hline Ilioinguinal nerve & $44(59)$ \\
\hline Iliohypogastric nerve & $9(12)$ \\
\hline Genitofemoral nerve & $15(20)$ \\
\hline \multicolumn{2}{|l|}{ Intraoperative complications, $\mathrm{n}(\%)$} \\
\hline Small bowel injury & $1(1)$ \\
\hline None & $73(99)$ \\
\hline \multicolumn{2}{|l|}{ Other intraoperative findings, $\mathrm{n}(\%)$} \\
\hline Meshoma & $23(31)$ \\
\hline Neuroma & $9(12)$ \\
\hline
\end{tabular}

Presented as mean \pm standard deviation or median [range] as appropriate. 


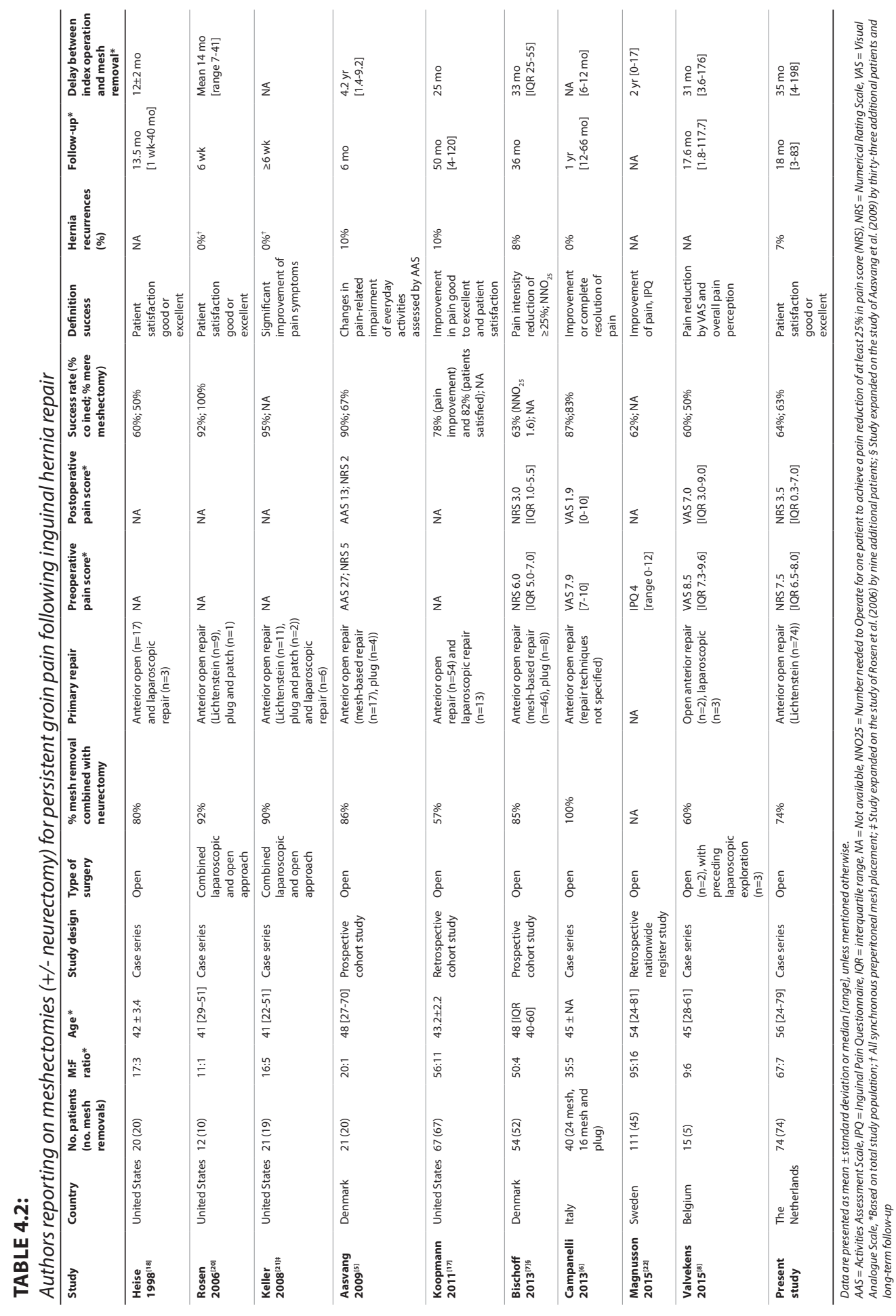




\section{Literature search}

The search strategy identified 109 papers on PubMed and 11 papers using Embase. Seven were considered eligible. Two additional papers were included after screening of reference lists. Five other studies reporting on mesh removal were not considered as no full text was available ${ }^{13}$, the meshectomy was solely performed either to facilitate a neurectomy ${ }^{14-16}$, or excluded patients with a pure nociceptive pain profile probably related to the mesh ${ }^{17,18}$. Randomized controlled trials were not identified. Table 4.2 summarizes the nine included studies, with a total of 278 meshectomies (largest series included 67 patients $\left.{ }^{19}\right)$. Most $(n=5)$ were retrospective case series. Published success rates ranged from 60 to $100 \%$, according to the definition of success. The majority of mesh removals were combined with a neurectomy (mean 82\%). Recurrence rate after meshectomy ranged from 0 to $10 \%$. Interesting, studies reporting no recurrences had previously performed preperitoneal mesh placement prior to meshectomy.

\section{DISCUSSION}

The present study reports on the largest series on meshectomy in patients suffering from mesh-related inguinodynia following a Lichtenstein procedure. Most cases were combined with a tailored neurectomy ${ }^{6}$. The results demonstrate that this type of groin pain surgery is effective in terms of pain relief in two thirds of the patients whereas complication rates were acceptable. As success rates following either pure mesh removal or mesh removal combined with neurectomy were similar, it may be concluded that removal of the foreign material per se can be very beneficial in selected patients. A 7\% hernia recurrence rate is acceptable but may become a point of concern if follow-up stretches beyond 18 months. The overview of the currently available literature may allow for a proposal of recommendations on meshectomy for inguinodynia.

A clear list of specifics for physicians (specialized in hernia treatment) allowing for the selection of appropriate patients for meshectomy would possibly increase remedial surgery success rates. However, characteristics suggesting that inguinodynia is exclusively mesh-related, and not nerve-related, are currently lacking. More than a decade ago, studies reported on the potential role of mesh as a cause for groin pain following inguinal repair ${ }^{20}$. A growing but still limited body of evidence indeed suggests that a meshectomy 
may be effective in most patients with suspected mesh-related inguinodynia (table 4.2). No specific attention was paid to define mesh-related pain in these studies. Our recommendations and patient selection mainly depend on the subjective evaluation of the patient's complaints and physical examination, guided by characteristics of mesh-related pain (figure 4.1). The importance of these aspects has been recognized by other hernia specialists $^{10,21}$. Characteristics of a mesh-related pain include sensations of a 'foreign body feeling' or tightness in groin area, pain aggravation during car driving or crossing legs, and pain relief by hip extension or supine position. Clues for mesh-related pain during physical examination are a painful deep palpation along the inguinal ligament $\geq 5 \mathrm{~cm}$, a diffuse pain and the lack of characteristics of neuropathic pain. Sometimes a meshoma can be palpable in non-obese patients. Of note, evaluation of patients should be performed by an experienced hernia surgeon ${ }^{12}$. The characteristics of mesh-related pain should be considered as a general tool of guidance rather than a checklist, since the value of history and physical examination is relative and not absolute.

It should be appreciated that most procedures of mesh removal required a combination with a selective neurectomy, whether or not neuropathic pain was suggested by the preoperative evaluation. If nerves are identified while in close contact to the mesh, a selective neurectomy may seem logical for various reasons. When the nerve is entrapped, the mesh cannot properly be removed without sacrificing the nerve. Moreover, it is often difficult to determine whether a nerve, even if it looks normal, in fact is damaged ${ }^{12}$. Leaving the nerve in situ would increase the risk on persistent postoperative pain. Retained nerves may also interfere with closing of the inguinal wall defect, possibly leading to a new onset type of neuropathic pain. This technical perspective has previously been recognized by other hernia surgeons dealing with mesh removal7-10, 19, 20, 22-24, who all performed a tailored or triple neurectomy in more than half (57\%) of the patients. A recent consensus algorithm also acknowledged this feature and advised a triple neurectomy within the operative field during meshectomy ${ }^{12}$. However, it is debatable whether a triple neurectomy is required and always possible in this patient group. In general, mesh removal is performed in a scarred operative field. It is unlikely that all three inguinal nerves may always be identified during remedial surgery ${ }^{7}$. Therefore, when a meshectomy is performed, a selective neurectomy may seem more practical.

It is assumed that groin pain can exclusively be of nociceptive or an inflammatory character. Previous studies demonstrated that the success of mesh removal is independent 
of performing a simultaneous neurectomy ${ }^{19}$. The results of the present study demonstrate identical successful outcomes in patients exclusively undergoing meshectomy (success rate $63 \%, n=19$ ) or a combined operation (success rate $64 \%, n=55$ ). This finding suggests that a neurectomy is not the most important factor determining pain relief after mesh removal. In fact, it supports the assumption that groin pain can also exclusively be of nociceptive or an inflammatory character caused by the presence of the mesh. This conclusion is in line with data from a multivariate analysis on predictors for success of meshectomies $^{19}$.

The experience of an attending surgeon may determine whether patients may benefit from mesh removal. This subjective selection process is also highlighted in international consensus statements and by other authors acknowledging that selection is the most important determinant of success ${ }^{10,12}$. As previously demonstrated, success rates of groin pain surgery clearly follow a learning curve ${ }^{6}$. The characteristics of mesh-related pain may aid other workers in this rather subjective selection process (figure 4.1).

One may question the rationale for using a local tissue repair technique in the prevention of a hernia recurrence following mesh removal as practiced in the present study. Generally accepted international guidelines on primary inguinal hernia repair dictate a mesh-based tension-free (i.e. Lichtenstein or endoscopic techniques) over a tension-repair (using own body tissue) ${ }^{5}$. In 10 years, a 12\% recurrence rate was reported in tension repairs compared to a $1 \%$ rate after mesh insertion ${ }^{25}$. Following this line of thought, insertion of a new mesh may have been preferred after inguinodynia-related mesh removal ${ }^{23}$. The literature on meshectomy demonstrates recurrence rates up to $10 \%$ (table 4.2). Patients who underwent a simultaneous laparoscopic mesh repair and meshectomy of the anterior placed mesh did not demonstrate recurrent hernias ${ }^{22,23}$. The present $7 \%$ recurrence rate in our study is lower than expected ${ }^{25}$. It is our experience that muscles and connective tissue forming walls and floor of the operation field had often scarred and hardened due to the presence of the mesh. Although a local tissue closure technique for recurrence prevention suffices in most patients, endoscopic insertion of a new mesh in case of a recurrence is advised.

Mesh removal is not routinely executed by all hernia surgeons. As a consequence, delay prior to mesh removal in patients suffering from a mesh-related pain is often long. International guidelines and algorithms on the management of postoperative chronic pain following inguinal hernia surgery do not provide recommendations for a pure mesh- 
related pain ${ }^{12,26,27}$. Others do consider a meshectomy in selected groups of patients but base their approach on one single study ${ }^{5,28}$. This reservation to operate is also reflected by a three years surgical delay in the present patient group. Our literature review also demonstrates a $>2$ years delay in most studies (table 4.2). A treatment algorithm that is based on available literature and the present study may aid in limiting this delay.

A recently published international consensus algorithm suggests that the present version should serve as a starting point for developing a dynamic algorithm ${ }^{12}$. As a consequence, it is proposed to adapt this algorithm based on recently available literature. First, if pain following a standard hernia repair persists after three to six months, a concise patient history and physical examination should indicate whether a patient suffers from neuropathic pain, from a nociceptive/inflammatory pain (either mesh-related or by other origins), or from pain due to a recurrent hernia. Additional imaging (i.e. MRI) preceding remedial surgery is not needed as low interobserver agreements for pathologic alterations following inguinal hernia repair were demonstrated ${ }^{21}$. Furthermore, MRI did not contribute to elucidating the underlying pathology in groin pain as it is not specific whereas differences in images of painful and pain-free operated groins were also not observed ${ }^{21,29}$. Second, when conservative treatments do not offer a significant pain relief, surgical interventions are considered. Neuropathic pain is treated by a triple or tailored neurectomy, whereas patients presenting with typical mesh-related symptoms (figure 4.1) may undergo a meshectomy.

A unique pathologic finding is a meshoma, which is becoming a more frequent issue $^{23}$. A meshoma may cause pain due to a volume effect or by mechanical pressure on inguinal structures. In the present study, the observed incidence was $31 \%$ (table 4.1 ). Of all patients whom underwent removal of a meshoma $(n=23)$, radiologic imaging performed in referring hospitals (CT- $(n=4)$ and MRI-scans $(n=3)$ were reviewed. Not one of these seven reports reported a meshoma. It was previously also found that imaging does not contribute to the diagnosis meshoma, since the majority of radiologists judge images of a meshoma as 'nonspecific postoperative changes, tissue densities or lymph nodes'11. These findings also highlight the role of extensive history taking and physical examination (figure 4.1). If a meshoma is suspected, the current consensus algorithm advises to consult a pain team. A subsequent partial or complete mesh removal is only suggested if the meshoma pain is refractory to conservative management ${ }^{12}$. In a previous study, factors determining surgery outcome were identified ${ }^{30}$. It was demonstrated that 
the presence and subsequent removal of a meshoma was significantly associated with a successful outcome (odds ratio 5.3) ${ }^{30}$. These findings support the notion that a meshoma legitimates removal without prior treatments by a pain specialist.

\section{Limitations}

A number of limitations are identified in the present study. Patients did undergo an additional long term follow-up by a standardized telephone interview but not by a physical examination. It is possible that asymptomatic recurrences have been missed although neither experienced nor reported by the patients. The question arises whether an asymptomatic recurrence is clinically relevant. Use and generation of a protocol for postoperative inguinal pain is challenging as a postherniorrhaphy inguinodynia population is highly heterogeneous. Discriminating between neuropathic and nociceptive/ inflammatory pain is often difficult as patients may demonstrate a combination of both (figure 4.1). In addition, pain specialists including surgeons treating this group of patients should ponder on the possibility of centralization of pain and the pitfall of cross-linking of inguinal nerves by variations in groin neuro-anatomy ${ }^{27}$. As a consequence, initiating a tailored treatment largely depends on the subjective evaluation of the attending hernia specialist. Lastly, the present conclusions are mainly based on retrospective case series including the present study. A set of definitive recommendations requires the execution of a proper systematic approach or meta-analysis.

\section{CONCLUSION}

In conclusion, the present study reports on the largest group of patients to date suffering from chronic mesh-related groin pain following Lichtenstein hernia repair. Based on a favourable outcome with acceptable complication rates and supported by previous studies, mesh removal combined with tailored inguinal neurectomy should be considered in patients unresponsive to other treatments. The characteristics of mesh-related pain may aid physicians in recognizing patients who may benefit from a meshectomy. 


\section{Acknowledgments}

The authors thank dr. J.P. Dieleman for her support and expertise regarding statistical analysis. We thank authors who responded to our e-mail and provided detailed data on their studies: prof. H. Kehlet, dr. C. Heise, dr. J. Starling, dr. E. Valvekens, dr. G. Sandblom and prof. G. Campanelli. 


\section{REFERENCE LIST}

1. Nienhuijs S, Staal E, Strobbe L, et al. Chronic pain after mesh repair of inguinal hernia: a systematic review. Am J Surg 2007;194(3):394-400.

2. Classification of chronic pain. Descriptions of chronic pain syndromes and definitions of pain terms. Prepared by the International Association for the Study of Pain, Subcommittee on Taxonomy. Pain Suppl 1986;3:S1-226.

3. Poobalan AS, Bruce J, Smith WC, et al. A review of chronic pain after inguinal herniorrhaphy. Clin J Pain 2003;19(1):48-54.

4. Franneby U, Sandblom G, Nordin P, et al. Risk factors for long-term pain after hernia surgery. Ann Surg 2006;244(2):212-9.

5. Simons MP, Aufenacker T, Bay-Nielsen M, et al. European Hernia Society guidelines on the treatment of inguinal hernia in adult patients. Hernia 2009;13(4):343-403.

6. Loos MJ, Scheltinga MR, Roumen RM. Tailored neurectomy for treatment of postherniorrhaphy inguinal neuralgia. Surgery 2010;147(2):275-81.

7. Aasvang EK, Kehlet $\mathrm{H}$. The effect of mesh removal and selective neurectomy on persistent postherniotomy pain. Ann Surg 2009;249(2):327-34.

8. Campanelli G, Bertocchi V, Cavalli M, et al. Surgical treatment of chronic pain after inguinal hernia repair. Hernia 2013;17(3):347-53.

9. Bischoff JM, Enghuus C, Werner MU, et al. Long-term follow-up after mesh removal and selective neurectomy for persistent inguinal postherniorrhaphy pain. Hernia 2013;17(3):33945.

10. Valvekens E, Nijs Y, Miserez M. Long-term outcome of surgical treatment of chronic postoperative groin pain: a word of caution. Hernia 2015;19(4):587-94.

11. Amid PK. Radiologic images of meshoma: a new phenomenon causing chronic pain after prosthetic repair of abdominal wall hernias. Arch Surg 2004;139(12):1297-8.

12. Lange JF, Kaufmann R, Wijsmuller AR, et al. An international consensus algorithm for management of chronic postoperative inguinal pain. Hernia 2014.

13. Arlt GD, Lamm T, Klosterhalfen B. Mesh removal in inguinal hernia repair. European Surgery - Acta Chirurgica Austriaca 2003;35(1):42-44.

14. Madura JA, Madura JA, 2nd, Copper CM, et al. Inguinal neurectomy for inguinal nerve entrapment: an experience with 100 patients. Am J Surg 2005;189(3):283-7. 
15. Amid PK, Chen DC. Surgical treatment of chronic groin and testicular pain after laparoscopic and open preperitoneal inguinal hernia repair. J Am Coll Surg 2011;213(4):531-6.

16. Delikoukos S, Fafoulakis F, Christodoulidis G, et al. Re-operation due to severe lateonset persisting groin pain following anterior inguinal hernia repair with mesh. Hernia 2008;12(6):593-95.

17. Vuilleumier H, Hubner M, Demartines N. Neuropathy after herniorrhaphy: indication for surgical treatment and outcome. World J Surg 2009;33(4):841-5.

18. Zacest AC, Magill ST, Anderson VC, et al. Long-term outcome following ilioinguinal neurectomy for chronic pain. J Neurosurg 2010;112(4):784-9.

19. Koopmann MC, Yamane BH, Starling JR. Long-term follow-up after meshectomy with acellular human dermis repair for postherniorrhaphy inguinodynia. Arch Surg 2011;146(4):427-31.

20. Heise CP, Starling JR. Mesh inguinodynia: a new clinical syndrome after inguinal herniorrhaphy? J Am Coll Surg 1998;187(5):514-8.

21. Aasvang EK, Jensen KE, Fiirgaard B, et al. MRI and pathology in persistent postherniotomy pain. J Am Coll Surg 2009;208(6):1023-8; discussion 28-9.

22. Rosen MJ, Novitsky YW, Cobb WS, et al. Combined open and laparoscopic approach to chronic pain following open inguinal hernia repair. Hernia 2006;10(1):20-24.

23. Keller JE, Stefanidis D, Dolce CJ, et al. Combined open and laparoscopic approach to chronic pain after inguinal hernia repair. Am Surg 2008;74(8):695-700; discussion 00-1.

24. Magnusson N, Gunnarsson U, Nordin P, et al. Reoperation for persistent pain after groin hernia surgery: a population-based study. Hernia 2015;19(1):45-51.

25. Dirksen CD, Beets GL, Go PM, et al. Bassini repair compared with laparoscopic repair for primary inguinal hernia: a randomized controlled trial. Eur J Surg 1998;164(6):439-47.

26. Alfieri S, Amid PK, Campanelli G, et al. International guidelines for prevention and management of post-operative chronic pain following inguinal hernia surgery. Hernia 2011;15(3):239-49.

27. Voorbrood CE, Burgmans JP, Van Dalen T, et al. An algorithm for assessment and treatment of postherniorrhaphy pain. Hernia 2015.

28. Miserez M, Peeters E, Aufenacker T, et al. Update with level 1 studies of the European Hernia Society guidelines on the treatment of inguinal hernia in adult patients. Hernia 2014;18(2):151-63. 
29. Kouhia S, Silvasti S, Kainulainen J, et al. Magnetic resonance imaging has no role in diagnosing the origin of pain in patients with overwhelmingly painful inguinal hernia. Hernia 2015;19(4):557-63.

30. Zwaans WA, Verhagen $\mathrm{T}$, Roumen RM, et al. Factors Determining Outcome After Surgery for Chronic Groin Pain Following a Lichtenstein Hernia Repair. World J Surg 2015;39(11):2652-62.

31. Baron R. Mechanisms of disease: neuropathic pain--a clinical perspective. Nat Clin Pract Neurol 2006;2(2):95-106.

32. Loos MJ, Roumen RM, Scheltinga MR. Classifying post-herniorrhaphy pain syndromes following elective inguinal hernia repair. World J Surg 2007;31(9):1760-5; discussion 66-7 



\section{CHAPTER 5}

Laparoscopic mesh removal for otherwise intractable inguinal pain following endoscopic hernia repair is feasible, safe and may be effective in selected patients

G.D. Slooter ${ }^{*}$

W.A.R. Zwaans ${ }^{*}$

C.W. Perquin

R.M.H. Roumen

M.R.M. Scheltinga

* shared first autiror

Surg Endosc 2017 [Epub ahead of print] 


\section{ABSTRACT}

Background: Laparoscopic inguinal hernia repair is preferred over an open technique because of reduced recovery time, favorable cost-effectiveness and less chronic postoperative inguinal pain. Nevertheless, some patients develop a nociceptive inguinal pain syndrome possibly related to presence of the mesh. This is the first study describing feasibility, safety and effectiveness of laparoscopic mesh removal in patients with chronic pain after endoscopic hernia repair.

Methods: Pre- and intraoperative data of chronic pain patients scheduled for endoscopic mesh removal were prospectively collected by a standard evaluation form. Long term efficacy was determined using pain scores, patient satisfaction and quality of life questionnaire. A Wilcoxon signed ranks test was used to determine significant differences between pre- and postoperative pain scores.

Results: Fourteen patients were studied (11 males, median 52 year). Type of primary hernia repair was TEP $(n=11)$ and TAPP $(n=3)$. The median interval between primary repair and endoscopic meshectomy was 28 months. Median operating time was 103 minutes. Conversion to open surgery was not required. One intraoperatively recognized bladder laceration was laparoscopically closed. Otherwise, no intraoperative or postoperative complications occurred. Ten months postoperatively (median, range 2-62 months), pain scores had dropped from 8 to $4(\mathrm{p}<0.01)$. Satisfaction was good or excellent in 10 patients. A recurrent hernia developed in two patients ( 3 and 8 months postoperatively) requiring an open mesh repair in one.

Conclusions: Laparoscopic mesh removal is a feasible, safe and effective option in selected patients with chronic groin pain after endoscopic hernia repair after endoscopic hernia repair in hands of an experienced surgeon. 


\section{INTRODUCTION}

Inguinal hernia repair is performed over 20 million times each year worldwide ${ }^{1}$ Both open (e.g. Lichtenstein repair) and endoscopic techniques are recommended by international guidelines ${ }^{2}$. However, endoscopic techniques including transabdominal preperitoneal repair (TAPP) and total extraperitoneal repair (TEP) are increasingly preferred over open techniques because of diminished recovery time and improved cost-effectiveness ${ }^{2}$. A recent report from Switzerland indicated that $42 \%$ of their inguinal hernias are currently treated using an endoscopic technique ${ }^{3}$. These increasing rates are likely representative of a similar trend in other European countries, if not worldwide.

A feared complication following hernia surgery is chronic postoperative inguinal pain (CPIP). CPIP rates after open repair are reported between 11 and $16.8 \%^{4-7}$. In contrast, recent studies reported a lower 6-12.4\% pooled incidence following endoscopic hernia repair ${ }^{4,8}$. Avoiding direct inguinal nerve contact and omitting fixation devices in the preperitoneal space are assumed to be major factors contributing to the benefits of endoscopic techniques regarding CPIP ${ }^{9}$. Nonetheless, still some patients suffer from unacceptable CPIP after endoscopic hernia repair.

Once patients are diagnosed with CPIP, various treatment modalities may be explored. If conservative approaches fail following open repair, patients may undergo inguinal neurectomy with or without removal of mesh $^{2}$. A recent study demonstrated that two of three CPIP patients following an open Lichtenstein procedure benefitted from a meshectomy ${ }^{10}$. However, removal of endoscopically inserted mesh is controversial as damage of vessels, gut or bladder are feared during both open and laparoscopic removal. As absolute numbers of patients suffering from CPIP following TEP or TAPP will further rise in the future due to the popularization of endoscopic techniques, it is worthwhile to explore the various possibilities of endoscopic mesh removal for CPIP. The present study describes feasibility and safety in the first series of patients with CPIP undergoing laparoscopic removal of laparoscopically inserted mesh. Secondly, effect on pain scores, patient satisfaction and quality of life were evaluated. 


\section{METHODS}

\section{Study design and participants}

The present study was conducted in SolviMáx, a centre of excellence for abdominal wall and groin pain. Patients were eligible if they had a history of endoscopic (TEP or TAPP) inguinal hernia repair and had undergone a laparoscopic meshectomy as remedial surgery for CPIP. Pain characteristics related to the presence of mesh after primary open repair were recently described ${ }^{10}$. These include sensations of a 'foreign body feeling' or tightness in groin area, pain aggravation during car driving, bending or crossing legs, and pain relief by hip extension or supine position. Clues for mesh-related pain during physical examination are a painful deep palpation along the inguinal ligament $\geq 5 \mathrm{~cm}$, a diffuse pain and the lack of sensory disturbances or triggerpoints. Sometimes a meshoma can be palpable in non-obese patients ${ }^{10}$. Identical criteria were used to determine whether a patient was suffering from mesh-related pain after endoscopic hernia repair.

Each patient with possible CPIP that is referred to our center via e-mail or letter is asked to complete a set of questionnaires on history, quality of life, previous physical examinations, imaging and prior therapies prior to consultation, After evaluation of these questionnaires, patients are invited to undergo a 30-minute outpatient assessment by one of four surgeons skilled in the management of abdominal wall and groin pain. Additional standard evaluation forms investigating history and physical examination are completed during this interview and stored in the hospital's electronic patient file. These prospectively collected preoperative data were used as baseline data.

Patient history includes questions such as presence of foreign body feeling in the groin or abdomen or if body position or activities provoke pain. Physical examination includes skin testing using a cotton swab, alcohol soaked gauze and skin pinching to exclude altered skin sensibility such as hypoesthesia, hyperesthesia or thermohypoesthesia indicating inguinal nerve injury.

If patients are likely to suffer from CPIP due to the preperitoneal mesh, they are informed on the specifics of surgery including potential complications such as damage to vessels, gut or bladder and the risk of conversion to an open procedure. Following an extensive consultation, patients are asked to sign an informed consent form. The daily board of the medical ethics committee of MMC reviewed the present research proposal 
and deemed that the rules laid down in the 'medical research involving human subjects act' do not apply on the present study. Patient data analysis was fully anonymized.

\section{Interventions}

All operations were performed under general anaesthesia in supine position. A urinary catheter was placed for bladder decompression but antibiotics were not administered. Palmer's point Veress needle access was used to create a pneumoperitoneum. Three trocars were placed in the ipsilateral upper abdomen. A disposable $12 \mathrm{~mm}$ Optiview trocar (Ethicon Endo-surgery, Cincinnati, OH, USA) placed through the rectus abdominis muscle allowed for a safe entry and facilitated mesh removal. A $10 \mathrm{~mm}$ trocar accommodating the camera was placed under direct vision in the midline and a $5 \mathrm{~mm}$ port was used laterally for a grasping instrument. Following simple adhesiolysis (3 of 14 patients), the position of the mesh was inspected (figure 5.1). The mesh was carefully dissected together with the overlying peritoneum, starting at the cranial or lateral border. Dissection was done with scissors and occasionally cautery. In 4 of 14 cases, inferior epigastric veins and arteries were clipped as they were tightly adhered to the mesh. The triangle of doom and the triangle of pain were subsequently explored. In the present series, the gonadal vessels were easily separated from the mesh as were illiac vein and artery. However, in none of these patients previous mesh fixation using tackers or alternative fixation material was performed. In male patients $(n=11)$, the spermatic duct that was often also tightly connected was dissected without harm. The median border of the mesh was freed from behind the medial umbilical fold via ipsilaterally positioned trocars. In the caudal plane, one had to dissect strictly adjacent to the mesh to avoid damage to the bladder. Finally, the mesh is released from the inner face of the pubic bone. Dissection is difficult as the mesh is firmly attached to this bony structure (figure 5.2). The mesh is cleaved and removed via the $12 \mathrm{~mm}$ trocar (figure 5.3). Peritoneal defects were not closed. All trocars were removed under direct vision. External fascias were closed using absorbable sutures whereas the skin was sutured intracutaneously. All patients stayed overnight because of long travelling distance and comfort but were discharged the day thereafter. 


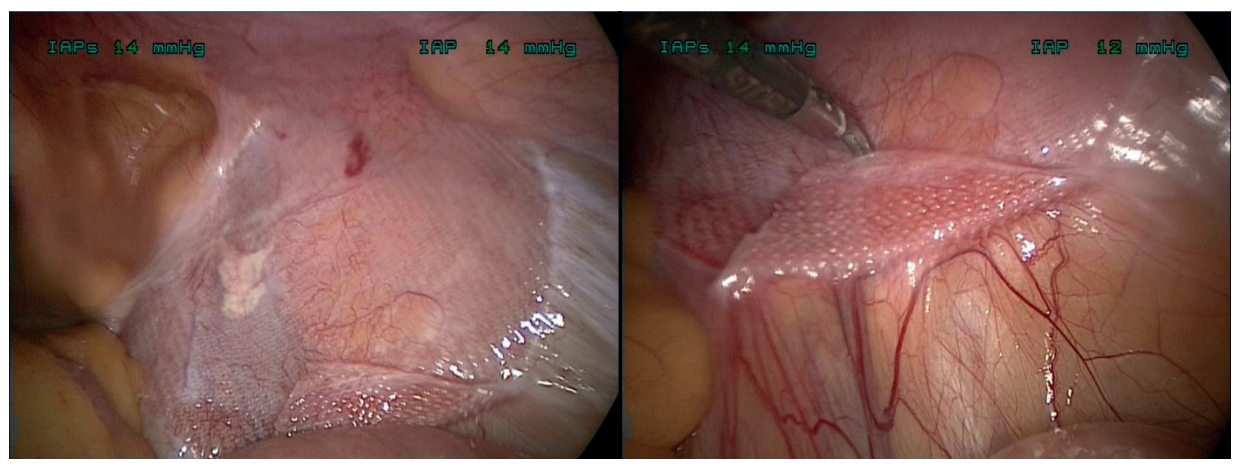

FIGURE 5.1:

Intraoperative view of a preperitoneal mesh in the right groin following a total extraperitoneal inguinal hernia repair.

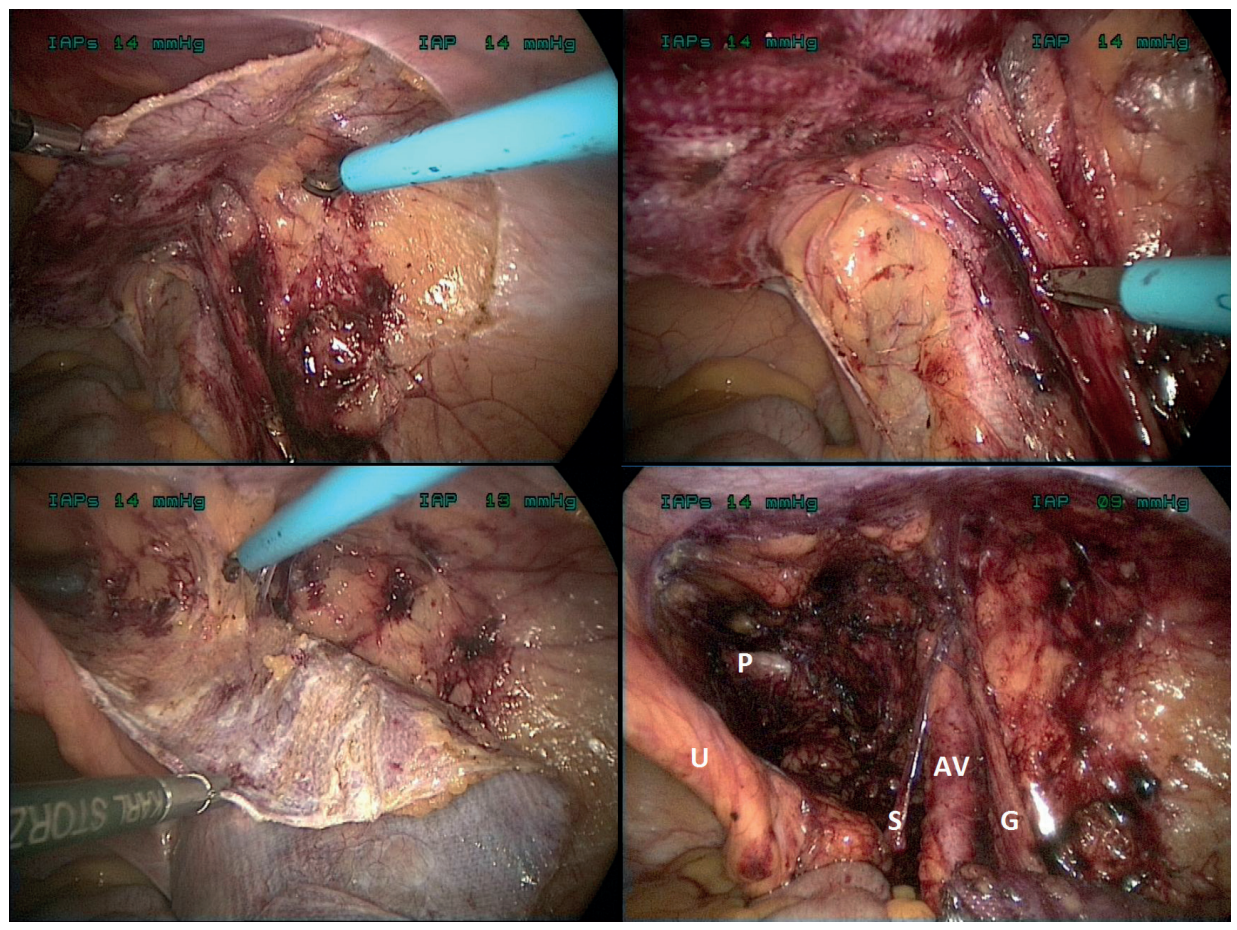

FIGURE 5.2:

Intraoperative view of laparoscopic mesh removal of a preperitoneal mesh in the right groin. After removal of the mesh one can identify the medial umbilical fold (U), the gonadal vessels $(G)$, the spermatic cord (S), the iliac vein and artery (AV) and the inner face on the pubic bone $(P)$. 


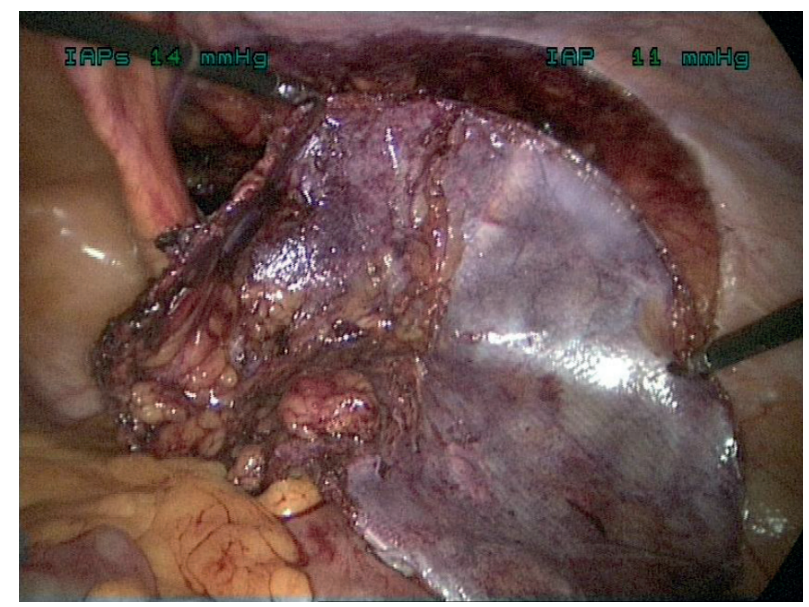

FIGURE 5.3:

Intra-abdominal view of the preperitoneal mesh fully removed from the right groin.

\section{Outcomes and data accrual}

Primary outcomes were feasibility and safety of laparoscopic mesh removal as reflected by intraoperative complications, conversion rates and adverse effects including hernia recurrences. Secondary outcome was the effect on pain using the Numerical Rating Scale (NRS), patient satisfaction (excellent-good-moderate-poor) and quality of life (truncated short-form health survey (SF-12) ${ }^{11}$. All patients were routinely followed up at the outpatient clinic six to eight weeks postoperatively. A final on-off telephone followup interview was performed aimed at determining long term patient satisfaction, pain scores and recurrent hernias.

Electronic patient files were used for extracting patient demographics such as age, gender, body mass index (BMI), medical history and year of primary hernia repair. Specifics of surgery included side of mesh removal, operation time, percentage of mesh removed and intraoperative complications.

\section{Statistical methods}

Data were collected and analyzed using the Statistical Package for the Social Sciences (SPSS, SPSS Inc. Chicago, IL, USA), version 22. Data were presented by descriptive statistics as medians with ranges. A Wilcoxon signed ranks test was performed to test for differences between preoperative and postoperative NRS pain scores. 


\section{RESULTS}

Between November 2011 and January 2017, fourteen patients underwent laparoscopic mesh removal for CPIP. Primary endoscopic hernia repairs (TEP, $n=11$, TAPP, $n=3$ ) were performed using a polypropylene, non-absorbable, large pore mesh between 1999 and 2015. Fixation devices were not used in this series. Baseline patient characteristics are presented in table 5.1. Before operation, the pain score was 8 [median, range 5-9]. Preoperative pain medication included opioids $(n=4)$ and gabapentinoids (pregabalin, $\mathrm{n}=1$ ). All patients had undergone one or more conservative treatments including injections of corticosteroids $(n=7)$ or local anaesthetic agents $(n=4)$, physical therapy $(\mathrm{n}=4)$ and transcutaneous electric nerve stimulation (TENS, $\mathrm{n}=2)$. Moreover, eight patients had received remedial surgery as a neurectomy $(n=6)$, adhesiolysis $(n=1)$ and adductor tenotomy $(\mathrm{n}=2)$ but without success.

\section{Outcomes and estimation}

Time between primary repair and meshectomy was 28 months [median, range 7-142]. Operating time was 103 minutes [median, range 44-204]. No conversions to open surgery were required. Significant bleeding did not occur. An intentional partial meshectomy was performed in two patients, whereas a concurrent neurectomy of the genitofemoral nerve was performed in two other patients. A meshoma, defined as folding of the inserted mesh into a mass-like density ${ }^{12}$, was not identified. In seven patients. however, the mesh was slightly folded. A small bladder laceration was repaired using two stitches in a female patient. Postoperatively she recovered uneventfully. Immediate postoperative complications such as bleeding, hematoma or infection were not observed. 


\section{TABLE 5.1:}

Baseline characteristics of patients undergoing laparoscopic mesh removal for chronic groin pain after total extraperitoneal repair or transabdominal preperitoneal repair $(n=14)$

\begin{tabular}{|c|c|}
\hline Age (in years) & $52[26-77]$ \\
\hline Sex (male:female) & $11: 3$ \\
\hline Body mass index $\left(\mathrm{kg} / \mathrm{m}^{2}\right)$ & $26.0[20.3-29.7]$ \\
\hline Primary hernia repair (TEP*:TAPP十) & $11: 3$ \\
\hline \multicolumn{2}{|l|}{ ASA $\neq$ classification $(n)$} \\
\hline 1 & 7 \\
\hline II & 6 \\
\hline III & 1 \\
\hline Patients with prior remedial surgeries (n) & 8 \\
\hline \multicolumn{2}{|l|}{ Prior imaging (n) } \\
\hline CAT scan & 7 \\
\hline MRI scan & 11 \\
\hline Abnormal position of mesh on imaging ( $n$ ) & 3 \\
\hline Preoperative NRS§ & $8.0[5.0-9.0]$ \\
\hline \multicolumn{2}{|l|}{ Preoperative SF-12|| scores } \\
\hline PCSף & 34 [17-57] \\
\hline MCS\# & $50[29-61]$ \\
\hline
\end{tabular}

\section{Physical examination}

Side (left:right)

History of mesh-related pain (n):

Foreign body feeling

Pain aggravation during car driving or crossing legs

Pain relief by hip extension or supine position

Mesh-related characteristics during physical examination (n)

Painful deep palpation along inguinal ligament

Absence of triggerpoints

Absence of sensory disturbances

${ }^{*} T E P=$ Total extraperitoneal repair; $+T A P P=$ Transabdominal preperitoneal repair; $¥ A S A=$ American Association of Anaesthesiologists; $§ N R S=$ Numerical Rating Scale; $\| S F-12=$ Short form health survey; IPCS = Physical component summary; \# MCS = Mental component summary 


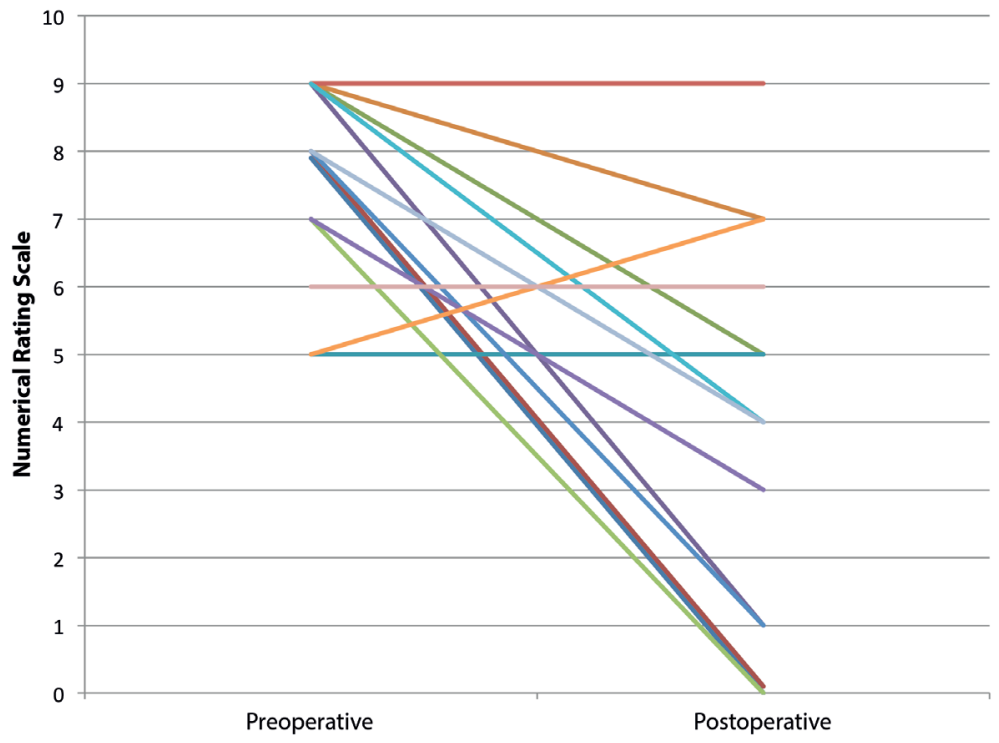

\section{FIGURE 5.4:}

Individual pain scores before and 10 months (median, range 2-62 months) after laparoscopic removal of preperitoneal mesh in patients with persistent pain after endoscopic primary hernia surgery

Follow-up was 8 months [median, range 2-62]. By then, pain scores had dropped from 8 to 4 [median $0-9$ ], $\mathrm{p}<0.01$. Nine patients experienced a $\geq 50 \%$ pain reduction (figure 5.4). Satisfaction was excellent in six, good in four, moderate in three and poor in one patient. Just one patient regretted surgery. An improved quality of life was demonstrated regarding both the physical component summary and the mental component summary (median, 45 versus 57 respectively). Only two patients still used pain medications. Two patients developed a recurrent hernia after three and eight months following meshectomy. In one, the recurrent hernia was repaired by Lichtenstein repair.

\section{DISCUSSION}

The present study is the first to report on laparoscopic mesh removal in a series of patients with chronic postoperative inguinal pain (CPIP) following an endoscopic hernia repair. The treatment effects of the meshectomy in this 14 patient series are promising. As 
no conversions were required and complications were minimal, it is concluded that in a selected group of patients laparoscopic mesh removal is feasible and safe in experienced hands and may potentially play a role in developing a treatment strategy for patients with mesh-related pain. The advent of two recurrences during the 8 months follow up is a point of concern. However, it must be appreciated that these recurrent hernias, if symptomatic, may effectively be treated using alternative open operative techniques including tissue repairs or mesh repairs.

An electronic search strategy aimed at identifying other studies on laparoscopic mesh removal for CPIP after hernia repair yielded two related papers. One patient with groin pain provoked by hip flexion after previous hernia surgery by a mesh \& plug technique successfully underwent a laparoscopic plug removal ${ }^{13}$. One article described a combined laparoscopic and open approach for mesh removal together with a triple neurectomy in six patients. Success rates on just laparoscopic mesh removals alone were not reported ${ }^{14}$. A $72 \%$ success rate (good or excellent) patient satisfaction after almost one year was reported in the present series.

A $21^{\text {st }}$ century hernia patient having access to all sources on the world wide web is well-informed and is knowledgeable on the phenomenon of mesh-related pain. As a consequence, a growing number of patients oppose to the use of meshes for hernia repair. CPIP is a highly invalidating complication and often leads to an inability to normally participate in society. However, rates of CPIP (and other complications) following endoscopic repair are relatively low compared to rates associated with open techniques ${ }^{2}$. Therefore, one may question whether this reluctance is justified. On the other hand, since the present new technique of mesh removal occurred to be feasible and safe, in hands of experienced and specialized surgeons, they may more readily accept a risk on CPIP knowing that remedial surgery can be effective. Of note, laparoscopic mesh removal is not advised as a first step in CPIP patient, as feasibility and safety are hitherto only established in the present group of selected patients in a highly experienced center.

One may seriously doubt whether total pain relief is only attained if all of the mesh is removed. In twelve patients, a complete meshectomy was performed whereas a partial removal was executed in two as symptoms were thought due to cranial portions of the mesh. One patient became free of pain whereas the second did not benefit from surgery. However, extend of mesh removal must be traded against risk of hernia recurrence and looming complications. Thus, a partial meshectomy may be effective and should 
pragmatically be considered, depending on the risk of recurrence, safety and grade of folding of the mesh. The results of this small series on CPIP are promising. Only one patient reported more pain following his meshectomy. At the preoperative consultation, a combined neuropathic and nociceptive pain was diagnosed leading to a mesh removal combined with a genitofemoral neurectomy. His more intense postoperative neuropathic complaints were classified as deafferentation pain. A restrained regimen was indicated by our pain team. Given the small number of patients in the present series, further studies of the effectiveness of laparoscopic meshectomy in a larger group of patients is desirable.

Can any laparoscopic surgeon perform a laparoscopic mesh removal? A profound understanding of normal anatomy is a key to success. A detailed exploration of both the triangle of doom and the triangle of pain are essential. In our series, the only intraoperative untoward event being a urinary bladder laceration was recognized and treated promptly leading to an uneventful recovery and a complete pain relief postoperatively. Surgeons may decide to waive mesh removal once (too) firm adhesions in the vicinity of these triangles may potentially lead to serious complications. Moreover, attachment to vessels or ureters are probably contraindications although these situations were not encountered in the present patient series. As previously stated, no fixation devices were used for primary endoscopic repair in our series. As the standard use of fixation methods highly depends on national preferences, mesh adherence to vascular structures or spermatic cord and its consequential difficulty of removal may vary considerably. As expected, the first author who performed all of the surgeries, attained more confidence over the years suggesting a gradual learning curve. He strongly feels that laparoscopic mesh removal should be strictly reserved to specialists having ample experience in laparoscopic hernia repairs.

Should each CPIP patient undergo laparoscopic mesh removal? A recent study described a set of nociceptive pain characteristics after open inguinal hernia repair justifying mesh removal ${ }^{10}$. Identical symptoms were considered as an indication for laparoscopic mesh removal in the present series leading to a similar success rate. It is thought that the mesh may exert comparable mechanical effects irrespective of mode of implantation, open or laparoscopic. Nevertheless, approximately 15\% of CPIP patients demonstrate a mesh-related pain after Lichtenstein repair ${ }^{10}$. Based on the present study encompassing just 14 patients over a 5 years' time period, mesh-related pain after 
endoscopic repair may be likely less prevalent than after open mesh surgery. However, no firm conclusions can be drawn concerning the incidence of this relative new entity.

Interestingly, gradations of a meshoma like a mass-like density that are traditionally associated with pain, were not identified in the present population compared to $31 \%$ following mesh removal after Lichtenstein repair ${ }^{10}$. However, slight creasing of the mesh was intraoperatively observed in seven patients. A meshoma following initial endoscopic hernia repair may look different compared to open repairs, as subtle effects as wrinkling or small folds of the preperitoneal mesh may have pressure and mass effects (figure 5.1). Limited mesh folding in the preperitoneal space is possibly related to less traction when compared to the groin region. Inadequate dissection of the preperitoneal space during the index surgery may have contributed to folding of the mesh. Nevertheless, even minimal folding may contribute to the same pain sensation.

In conclusion, the present case series is the first to demonstrate that laparoscopic mesh removal is a feasible and safe technique that can be considered for selected patients with nociceptive CPIP after endoscopic hernia repair. This type of care should only be performed by hernia surgeons in expert centers, as results heavily depend on patient selection. Caution is warranted for extrapolation of these results in general hernia centers. Larger series are necessary to study the treatment effects and to identify factors associated with a favorable prognosis. 


\section{REFERENCES}

1. Kingsnorth A, LeBlanc K. Hernias: inguinal and incisional. Lancet 2003; 362(9395):1561-71.

2. Simons MP, Smietanski M, Bonjer HJ, et al. World guidelines for groin hernia management. 2016.

3. Tschuor C, Metzger J, Clavien PA, et al. Inguinal hernia repair in Switzerland. Hernia 2015; 19(5):741-5.

4. Koning GG, Wetterslev J, van Laarhoven CJ, et al. The totally extraperitoneal method versus Lichtenstein's technique for inguinal hernia repair: a systematic review with meta-analyses and trial sequential analyses of randomized clinical trials. PloS one 2013; 8(1):e52599.

5. Perkins FM, Kehlet $\mathrm{H}$. Chronic pain as an outcome of surgery. A review of predictive factors. Anesthesiology 2000; 93(4):1123-33.

6. Nienhuijs S, Staal E, Strobbe L, et al. Chronic pain after mesh repair of inguinal hernia: a systematic review. Am J Surg 2007; 194(3):394-400.

7. Simons MP, Aufenacker T, Bay-Nielsen M, et al. European Hernia Society guidelines on the treatment of inguinal hernia in adult patients. Hernia 2009; 13(4):343-403.

8. Aasvang E, Kehlet H. Chronic postoperative pain: the case of inguinal herniorrhaphy. Br J Anaesth 2005; 95(1):69-76.

9. Grant AM, Scott NW, O’Dwyer PJ. Five-year follow-up of a randomized trial to assess pain and numbness after laparoscopic or open repair of groin hernia. Br J Surg 2004; 91(12):15701574.

10. Zwaans WA, Perquin CW, Loos MJ, et al. Mesh Removal and Selective Neurectomy for Persistent Groin Pain Following Lichtenstein Repair. World J Surg 2017; 41(3):701-712.

11. Ware J Jr, Kosinski M, Keller SD. A 12-Item Short-Form Health Survey: construction of scales and preliminary tests of reliability and validity. Med Care 1996; 34(3):220-33.

12. Amid PK. Radiologic images of meshoma: a new phenomenon causing chronic pain after prosthetic repair of abdominal wall hernias. Arch Surg 2004; 139(12):1297-8.

13. Ohkura $Y$, Haruta S, Shinohara H, et al. Laparoscopic plug removal for femoral nerve colic pain after mesh \& plug hernioplasty. BMC Surg 2015; 15:64.

14. Keller JE, Stefanidis D, Dolce CJ, et al. Combined open and laparoscopic approach to chronic pain after inguinal hernia repair. Am Surg 2008; 74(8):695-700; discussion 700-1. 313-7. 



\section{CASEI}

A remarkable pitfall in inguinal open mesh herniorrhaphy

W.A.R. Zwaans

M.R.M. Scheltinga

R.M.H. Roumen

Gastrointest Dig Syst 2015; 5: 5 


\section{INTRODUCTION}

Inguinal herniorrhaphy is one of the most frequently performed procedures in general surgery ${ }^{1}$. In Europe, the majority of adult inguinal hernias is still repaired by open mesh approach such as a Lichtenstein technique ${ }^{2}$. This evidence based operation is thoroughly investigated and universally recommended for unilateral hernia repair by international guidelines ${ }^{2}$. Although the sequence of surgical steps of this Lichtenstein approach is well documented, pitfalls are potentially looming. Results of a questionnaire completed by Dutch surgeons and surgical residents demonstrated that more than half actually does not implement all of the surgical steps as described by the Lichtenstein Hernia Institute ${ }^{3}$. Specific attention should be paid to the inguinal nerves and the spermatic cord to prevent untoward damage leading to persistent postoperative pain. Therefore, deviating from this suggested road map may result in specific complications.

A patient was referred to SolviMáx, our 'centre of excellence for chronic abdominal wall and groin pain' because of persistent inguinodynia after a Lichtenstein repair. A surgical exploration revealed a spermatic cord that was divided by the mesh, supposedly during the primary repair. As a consequence, the patient had developed severe neuropathic pain originating from a damaged genitofemoral nerve. Following a tailored neurectomy, he became pain free.

\section{CASE REPORT}

A 52-year-old man presented with severe groin pain that started immediately after a Lichtenstein hernia repair six months previously in another hospital. His level of pain as scored using a Numerical Rating Scale was 8-9 on a 0-10 scale. Pain worsened during exercise, during flexion of the hip, during micturition and following sexual activity (dysejaculation) $)^{4}$. At physical examination, signs of neuropathic pain (hyperpathy, allodynia) in the right groin area as well as the area of the right testicle and right upper leg were found. Extreme tenderness was present along the entire length of the spermatic cord. Slight traction onto the scrotum even intensified this severe pain. In addition, hypoalgesia was present in the right hemiscrotum and medial upper leg. The patient was diagnosed with an entrapment of the genital branch of the genitofemoral nerve. A 
lidocaine injection into the most painful spot in the groin offered a $50 \%$ albeit temporary relief.

Following informed consent, an open exploration under general anaesthesia via the prior groin incision demonstrated a congested and obstructed but small spermatic cord. After releasing a ventrolateral portion of the cord, the mesh was lifted exposing a second portion of the spermatic cord. The mesh apparently had split the duct into two halves. In these remains, an oedematous genitofemoral nerve was identified that was trapped between the mesh and the pubic bone. A tailored neurectomy of the nerve was performed and the mesh was partially removed. However, the medial part of the mesh was left in situ to prevent a recurrence. Postoperatively, the patient was immediately pain free and recovered uneventful. Histopathological examination of the resected specimen did not showed traumatic neuroma formation of the nerve.
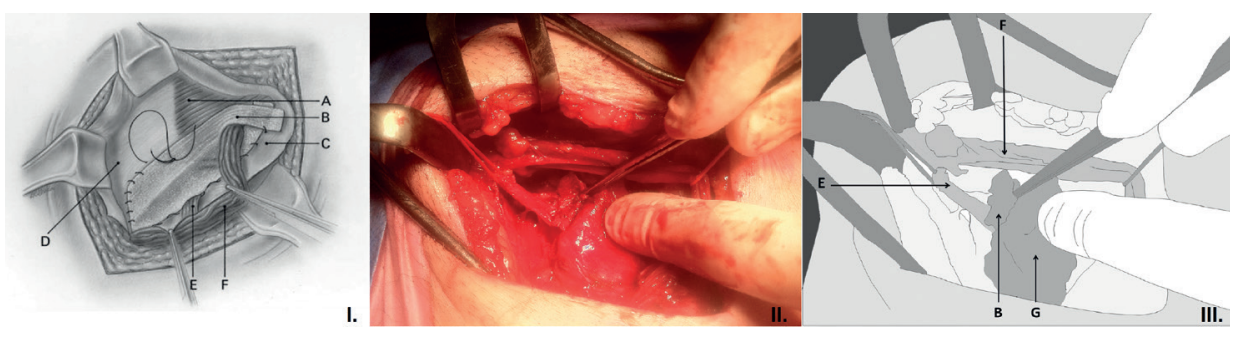

\section{FIGURE I.1:}

I. Illustration representing preliminary tension-free procedure according to Lichtenstein (reprinted from: Amid, P.K., Lichtenstein tension-free hernioplasty: its inception, evolution, and principles. Hernia, 2004. 8(1):1-7, with permission from Springer Science and Business Media). II./III. View during groin exploration (mirrored).

The spermatic cord (F) appears to be split. The mediodorsal part, containing the genital branch of the genitofemoral nerve $(E)$ is crushed by the mesh $(G)$ that is fixed onto the pubic bone. The ventrolateral portion of the cord $(E)$ is pulled cranially. The mesh is retracted by the forceps $(G)$ while the surgeon's finger is on the remaining portion of the mesh (B). A. Internal oblique muscle, B. Polypropylene mesh, $C$. Inguinal ligament, D. Internal oblique aponeurosis, E. Lesser cord containing the genital nerve, F. Spermatic cord, G. Polypropylene mesh infiltrated by the patients tissue. 


\section{DISCUSSION}

A host of papers alert surgeons regarding difficulties that may be encountered during a Lichtenstein hernia repair. Known pitfalls include a too tight mesh fixation, periostitis by placement of sutures too close to the pubic bone and ilioinguinal nerve damage. Amid (2004) described the key principles of the Lichtenstein tension-free herniorrhaphy as a step-by-step guideline for surgeons ${ }^{5}$. His main message was that, despite the procedure is easy to learn, perform and teach, some key principles must always be respected in order to obtain success ${ }^{5}$. A recent report, however, suggests that just half of the surgeons followed all of these consecutive steps ${ }^{3}$. If these Lichtenstein guidelines are ignored, some form of groin pain may potentially occur.

In the present case, we found a spermatic cord that was split during the index surgical procedure, while the mesh was placed over the medial portion causing severe inguinodynia. This cause of groin pain is, to our knowledge, not earlier described. In 1984, when the first Lichtenstein first tension-free herniorrhaphy was proposed, description of the technique did not allow for a careful dissection of the genitofemoral nerve ${ }^{5}$. It was even advised to separate the genital branch and external spermatic vessels from the rest of the cord. In fact, attention was mainly paid to preservation of the duct, the testicular artery and the ilioinguinal nerve. As a consequence, the so-called 'lesser cord' (genitofemoral nerve and external spermatic vessels) was at risk of being entrapped underneath the mesh that was placed over the pubic bone. This approach may have resulted in persistent inguinodynia in some patients ${ }^{5}$. This splitting technique was subsequently abandoned.

Identification and preservation of all three inguinal nerves (ilioinguinal, iliohypogastric and genitofemoral nerve) are nowadays considered important steps in the prevention of postoperative groin pain. Wijsmuller and co-authors also concluded that most respondents of a questionnaire on hernia surgical techniques were not sufficiently experienced with the neuro-anatomy of the groin ${ }^{3}$. Incomplete knowledge of the inguinal anatomy can result in debilitating groin pain due to nerve injury. The present case demonstrates that inadequate freeing of the entire spermatic cord prior to dorsal mesh placement can result in separation of the genitofemoral nerve from this cord, leaving it onto the pubic region. The mesh is then placed (and often fixed) onto the nerve possibly leading to an immediate postoperative severe neuropathic groin pain. 


\section{CONCLUSION}

Performing a Lichtenstein hernia repair requires meticulous dissection according to a set of key steps including nerve identification. If not, inguinodynia may occasionally occur. The number of patients with persistent groin pain following a standard open repair is still over 10 percent $^{6}$. If, before mesh placement, the spermatic cord is not complete freed from its surroundings, entrapment of the genital branch of the genitofemoral nerve may occur that most likely results in persistent groin pain.

\section{REFERENCES}

1. Sarosi GA, Wei Y, Gibbs JO, et al. A clinician's guide to patient selection for watchful waiting management of inguinal hernia. Ann Surg 2011;253(3):605-10.

2. Simons MP, Aufenacker T, Bay-Nielsen M, et al. European Hernia Society guidelines on the treatment of inguinal hernia in adult patients. Hernia 2009;13(4):343-403.

3. Wijsmuller AR, Lange JF, van Geldere D, et al. Surgical techniques preventing chronic pain after Lichtenstein hernia repair: state-of-the-art vs daily practice in the Netherlands. Hernia 2007;11(2):147-51.

4. Verhagen T, Loos MJ, Scheltinga MR, et al. Surgery for chronic inguinodynia following routine herniorrhaphy: beneficial effects on dysejaculation. Hernia 2016;20(1):63-68.

5. Amid PK. Lichtenstein tension-free hernioplasty: its inception, evolution, and principles. Hernia 2004;8(1):1-7.

6. Nienhuijs S, Staal E, Strobbe L, et al. Chronic pain after mesh repair of inguinal hernia: a systematic review. Am J Surg 2007;194(3):394-400. 



\section{CHAPTER 6}

Factors determining outcome after surgery for chronic groin pain following a Lichtenstein hernia repair

W.A.R. Zwaans

T. Verhagen

R.M.H. Roumen

M.R.M. Scheltinga

World J Surg 2015; 39: 2652-2662 


\section{ABSTRACT}

Background: Some patients develop chronic groin pain after a Lichtenstein hernia repair. Previous studies have demonstrated beneficial effects of removal of entrapped inguinal nerves or a meshomectomy in patients with chronic pain after open inguinal hernia mesh repair. Factors determining success following this remedial surgery are unknown. Aim of the study was to identify potential patient or surgery related factors predicting the surgical efficacy for inguinodynia following Lichtenstein repair.

Methods: Consecutive adult patients with a history of persistent pain following Lichtenstein repair who underwent remedial surgery were analyzed using univariate analysis. Significant confounders $(\mathrm{p}<0.05)$ were combined in a multivariate logistic regression model using a backward stepwise regression method.

Results: A total of 136 groin pain operations were available for analysis. Factors contributing to success were removal of a meshoma (OR 4.66) or a neuroma (OR $5.60)$ and the use of spinal anaesthesia (OR 4.38). In contrast, female gender (OR 0.30 ) and preoperative opioid use (OR 0.38 ) were significantly associated with a less favourable outcome. Using a multivariate analysis model, surgery under spinal anaesthesia (OR 4.04), preoperative use of opioids (OR 0.37) and meshoma removal (OR 5.31) greatly determined surgical outcome.

Conclusions: Pain reduction after remedial surgery for chronic groin pain after Lichtenstein repair is more successful if surgery is performed under spinal anaesthesia compared to general anaesthesia. Removal of a meshoma must be considered as success rates are optimized following these measures. Patients using opioids preoperatively have less favourable outcomes. 


\section{INTRODUCTION}

A recurrent inguinal hernia is a rarity since prosthetic material is routinely used for open inguinal hernia repair according to Lichtenstein. Therefore, clinical interest and research nowadays focus on issues related to persistent postoperative groin pain ('inguinodynia'). Inguinodynia is an untoward and incapacitating complication of herniorrhaphy that affects more than $10 \%$ of the patients following standard open mesh repair ${ }^{1}$. This percentage is likely an underestimation as the majority of patients suffering from persistent pain is not referred for additional therapy ${ }^{2-4}$. Moreover, surgeons and residents also report that the incidence of persistent groin pain following open repair is probably rated too $l_{0}$.

Once diagnosed with inguinodynia, a host of conservative treatments may be offered although often to no avail ${ }^{6}$. If pain is recalcitrant and deemed neuropathic in origin, a tailored neurectomy (removal of one or more presumed entrapped inguinal nerves) or a triple neurectomy (standard excision of all three inguinal nerves) may cure the pain ${ }^{6-8}$. Occasionally, partial or complete removal of mesh is additionally required. These surgical procedures are safe and reasonably effective $e^{6,9,10}$ as significant pain relief is achieved in over two thirds of the patients $s^{9,11-14}$. However, type and extend of surgery are often determined by the preference and experience of the attending hernia surgeon.

Risk factors associated with the onset of inguinodynia after the primary Lichtenstein hernia repair are patient or surgery specific ${ }^{15}$. Young age, the presence of pre-existent and immediate postoperative pain and incomplete identification of all three inguinal nerves during surgery were identified as risk factors for persistent postoperative pain $^{1,5,15-17}$. However, detailed studies on factors that influence the operative success of remedial surgery for Lichtenstein associated inguinodynia are lacking. To our knowledge, identification of variables determining surgical success has not been performed. Therefore, selection of appropriate operative candidates is currently based on subjective findings and not evidence based. Aim of the present study was to identify potential patient or surgery related factors predicting a successful outcome of surgery for inguinodynia after a Lichtenstein inguinal hernia repair. 


\section{MATERIALS AND METHODS}

\section{Design, setting and participants}

In this retrospective non-experimental study, electronic files of patients with a history of Lichtenstein hernia repair that underwent open surgery for persistent groin pain between the $1^{\text {st }}$ of January 2000 and the $31^{\text {st }}$ of October 2013 in the SolviMáx Centre for Abdominal Wall and Groin Pain, Máxima Medical Centre (MMC, the Netherlands) were reviewed. Potential study subjects were identified via an exclusive operative code that was previously assigned to any surgical procedure involving removal of inguinal nerves or mesh for inguinodynia as reported ${ }^{14}$.

Study inclusion criteria were chronic ( $\geq 3$ months) inguinodynia after a primary open Lichtenstein hernia repair, age $\geq 18$ year and open remedial surgery for groin pain (i.e. a neurectomy of one or more nerves and/or partial or complete mesh removal) via the original incision that was previously used for the Lichtenstein hernia repair. Exclusion criteria were pain following other (e.g. laparoscopic) types of hernia surgery, malignancy, cognitive impaired individuals, meralgia paresthetica and signs of groin infection at the time of surgery. Patients who underwent groin pain surgery more than once for the same side were also excluded from analysis to avoid multiple inclusions of patients' potential determinants. Patients who underwent bilateral surgery $(n=5)$ were not excluded, but each side was analyzed separately. For matters of clarity, results are presented as number of patients.

Demographics, clinical data (table 6.1) and operative notes were anonymized and entered in a separate database allowing further analysis. Specifics of the remedial surgical procedure were studied in detail. Pain scores using a Numerical Rating Score (NRS, 0 (no pain) to 10 (unbearable)) that were obtained during the preoperative outpatient department evaluation were used for analysis of surgical efficacy. All patients received a control appointment, usually 6 weeks postoperatively. Additional controls were performed if deemed required thereafter. In the period between January and March, 2014, the present health status was investigated using a telephone interview. A standard set of questions determined long-term outcome and current levels of pain during this interview. If preoperative pain scores were available, levels of pain as recalled by the patient were obtained during this interview allowing analysis of potential recall bias effects. A successful operation was defined as $\geq 50 \%$ pain reduction compared to the 
preoperative pain score ${ }^{18}$. The MMC's ethics committee deemed that evaluation of the study protocol was not necessary since the present analysis was considered evaluation of patient related outcome measures (PROM).

\section{TABLE 6.1:}

Patient factors and surgical factors that were analyzed to determine success following an operation for inguinodynia after previous Lichtenstein repair

\begin{tabular}{|c|c|}
\hline Patient related factors & Surgery related factors \\
\hline Age & Operation time \\
\hline Gender & Surgeon \\
\hline Length & Date primary inguinal hernia repair \\
\hline Weight & Inguinal nerves removed during pain surgery \\
\hline $\mathrm{BMI}^{*}$ & Date remedial surgery \\
\hline Smoking status & Identification of inguinal nerves \\
\hline Profession & Type of anaesthesia \\
\hline Medical history & Histopathological formation of neuroma \\
\hline Number of prior groin interventions/operations & Macroscopic formation of meshoma ${ }^{\S}$ \\
\hline Use of analgesics prior to groin pain surgery & Intraoperative complications \\
\hline ASA classification $^{\dagger}$ & 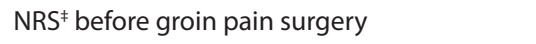 \\
\hline \multicolumn{2}{|l|}{ Radiologic examination preceding remedial surgery } \\
\hline \multicolumn{2}{|l|}{ Effectiveness of nerve blocks before remedial surgery } \\
\hline \multicolumn{2}{|l|}{ Presence of preoperative dysejaculation } \\
\hline \multicolumn{2}{|l|}{ Presence of preoperative testicular pain } \\
\hline \multicolumn{2}{|l|}{ Preoperative suspicion of affected nerves } \\
\hline \multicolumn{2}{|l|}{ Medial/lateral hernia } \\
\hline Side hernia & \\
\hline
\end{tabular}

${ }^{*} B M I=$ Body Mass Index $;{ }^{+}$ASA = American Society of Anaesthesiologists; ${ }^{*}$ NRS = Numerical Rating Score;

${ }^{\S}$ Meshoma $=$ folding and wrinkling of the inserted mesh, varying from a mass-like density to more subtle effects of mesh wrinkling or fibrosis ${ }^{19,20}$, as determined by the operating surgeon. 


\section{Calculations and statistical analysis}

Data were analyzed using SPSS version 22.0 software (SPSS Inc., Chicago, IL, US). A Wilcoxon signed-rank test was used to determine the difference between pain before and after the remedial groin pain surgery. Pain scores in the preoperative phase as recalled by the patient during the telephone interview were used if preoperative NRS scores were missing in the electronic patient file. Subsequently, the outcome measure was dichotomized by means of effectiveness (more or less than 50\% pain reduction).

Patient characteristics and intraoperative variables were tested for confounding properties by univariate analysis. Significant confounders $(\mathrm{p} \leq 0.05)$ were included as covariates in a multivariate logistic regression model as a means to determine the association between potential prognostic variables and outcome measures. The corresponding odds ratios (OR) and 95\% confidence intervals (95\%CI) were calculated. An OR $>1.0$ indicates a higher chance on a successful operation whereas an OR $<1.0$ indicates a lower chance. A likelihood ratio backward (rather than a forward) stepwise regression method was utilized because of a limited chance on suppressor effects and a lower risk of on a Type II error. Normally distributed data were presented as mean $( \pm S D)$. Nonparametric data were expressed as median with associated interquartile ranges.

Since preoperative pain scores were obtained both from the electronic patient files (prospectively acquired) as well as during the telephone interview (retrospectively acquired), a paired t-test was performed to investigate the potential presence of recall bias. A separate analysis in the successful and the failure group detected potential differences between these prospectively and retrospectively obtained preoperative scores. Baseline characteristics of patients with an incomplete primary outcome measure data set were also compared to the group with a complete set of pain scores. These tests were performed as an internal check for presence of information bias. Categorical demographic variables were compared using the Chi-square test. In contrast, continuous data were analyzed using the independent t-test or Mann-Whitney $U$ test when appropriate.

A variable was only tested for significance if at least $70 \%$ of the data was complete. In contrast, categorical variables in groups harbouring a limited number of individuals $(\mathrm{n} \leq 5)$ were omitted from analysis. Since spinal anaesthesia failed in four analyzed patients necessitating general anaesthesia, these patients were added to the category 'general anaesthesia. 
Low and high NRS were calculated by 25 percent and 75 percent interquartile ranges of the study population, respectively. Statistical significance for the multivariate logistic binary regression was set at $\mathrm{p} \leq 0.05$.

\section{RESULTS}

\section{Patient selection}

A total of 330 operations for inguinal hernia related pain were performed in SolviMáx during the almost 14 year time period (2000-2013). A total of 153 patients fulfilled all inclusion criteria. Four different types of operations were performed (figure 6.1, table 6.2). A neurectomy was performed in more than half of operations $(52 \%, 79 / 153)$. A neurectomy combined with limited mesh removal (necessary to properly remove nerves) occurred in $16 \%(25 / 153)$ of the population. In addition, $24 \%(37 / 153)$ underwent a neurectomy combined with mesh removal whereas just 8\% (12/153) received mesh removal exclusively. Remedial groin pain surgery was by definition effective in $54 \%$ $(n=74)$ of the patients. Results of additional surgery in unsuccessful patients are not presented in this report.

Demographics of these four patient groups are depicted in table 6.2. Mean age was 55 \pm 13 years (90\% males, $\mathrm{n}=138$ ). Median follow-up period was more than two years $(27$ months, interquartile range 16-51). As follow-up data were not available in 17 patients (died, $\mathrm{n}=5$; no contact, $\mathrm{n}=11$; refused participation, $\mathrm{n}=1$ ), results are based on $89 \%$ $(136 / 153)$ of the included patients. 


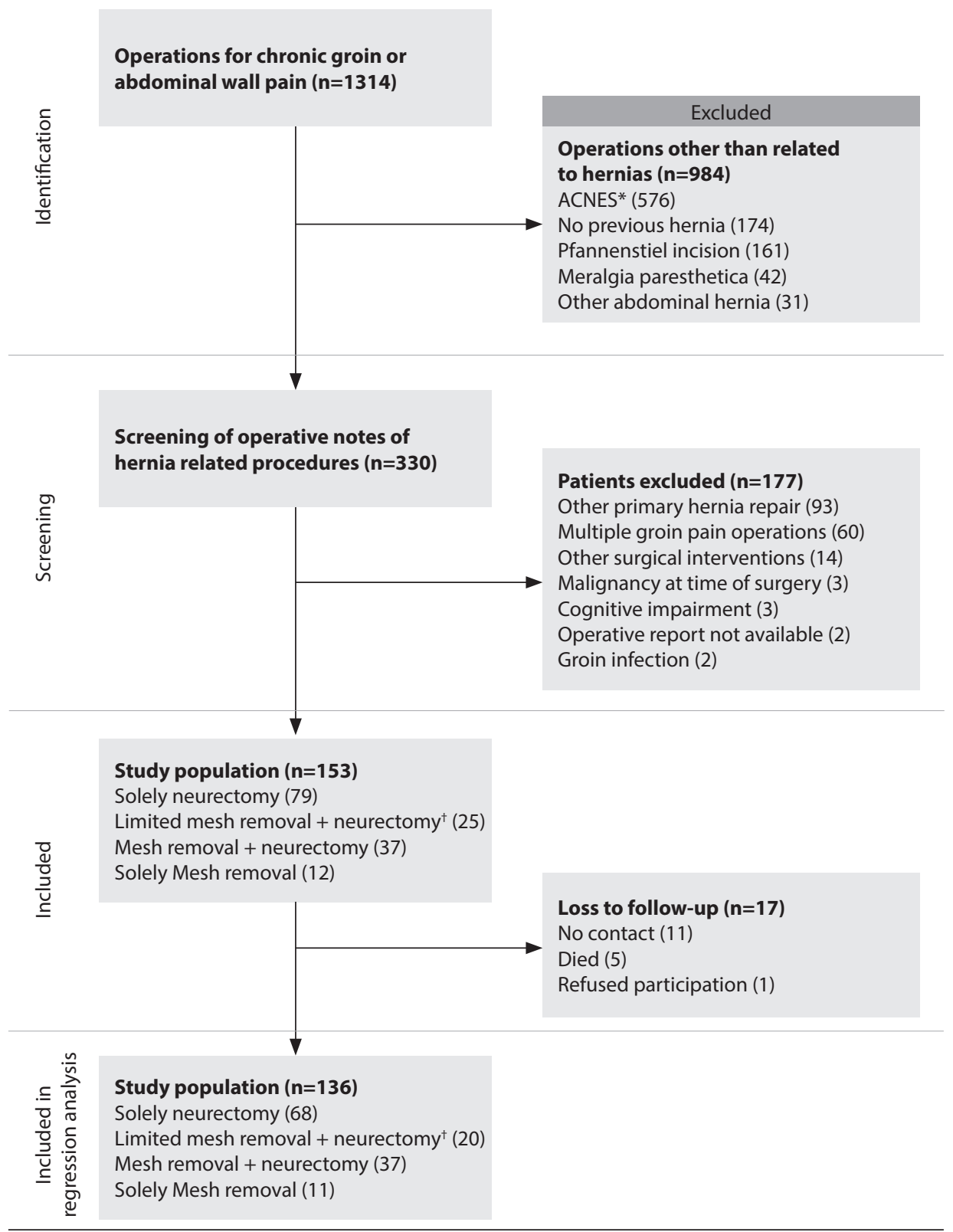

FIGURE 6.1:

Flowchart of selection process.

* ACNES = Abdominal Cutaneous Nerve Entrapment Syndrome; ${ }^{+}$Patients undergoing neurectomy combined with limited mesh removal, necessary to properly remove the nerve. 
TABLE 6.2:

Characteristics of patients undergoing various types of remedial groin surgery for pain after Lichtenstein repair

\begin{tabular}{|c|c|c|c|c|c|}
\hline Characteristic & Neurectomy & $\begin{array}{l}\text { Neurectomy } \\
+ \text { LMR }^{*}\end{array}$ & $\begin{array}{l}\text { Neurectomy } \\
+\mathrm{MR}^{+}\end{array}$ & Mesh removal & Total \\
\hline $\mathrm{n}$ & 79 & 25 & 37 & 12 & 153 \\
\hline Age (yr) & $54 \pm 14$ & $55 \pm 13$ & $55 \pm 13$ & $56 \pm 9$ & $55 \pm 13$ \\
\hline Gender (M/F) & $73 / 6$ & $22 / 3$ & $32 / 5$ & $11 / 1$ & $138 / 15$ \\
\hline Delay (mo) & 30 [13-58] & 32 [14-70] & 35 [16-82] & 37 [17-96] & 32 [14-64] \\
\hline Follow-up (mo) & $30[17-51]$ & 45 [17-85] & 20 [9-27] & $21[7-54]$ & $27[16-51]$ \\
\hline Length (cm) & $176 \pm 8$ & $177 \pm 7$ & $177 \pm 7$ & $181 \pm 9$ & $177 \pm 8$ \\
\hline Weight (kg) & $81 \pm 11$ & $82 \pm 16$ & $83 \pm 12$ & $91 \pm 11$ & $82 \pm 12$ \\
\hline Body Mass Index $\left(\mathrm{kg} / \mathrm{m}^{2}\right)$ & $26 \pm 3$ & $26 \pm 4$ & $27 \pm 4$ & $28 \pm 3$ & $26 \pm 4$ \\
\hline Operation time (min) & $39[31-52]$ & 43 [33-53] & $70[58-85]$ & $72[46-90]$ & 48 [34-65] \\
\hline Neurectomy $\mathrm{y}^{\ddagger}$ (n) & & & & & 92 \\
\hline N. Ilioinguinal & $85(67)$ & $88(22)$ & $84(31)$ & & 78 \\
\hline N. lliohypogastric & $29(23)$ & $33(8)$ & $14(5)$ & & 24 \\
\hline N. Genitofemoral & $39(31)$ & $21(5)$ & $27(10)$ & & 30 \\
\hline Side operation (L/R) & $32 / 47$ & $8 / 17$ & $18 / 19$ & $1 / 11$ & $59 / 94$ \\
\hline
\end{tabular}

Data presented as means $\pm S D$ or median with associated interquartile ranges, as appropriate. "Neurectomy $+L M R=$ Patients undergoing neurectomy combined with limited mesh removal, necessary to properly remove the nerve; ${ }^{+}$Neurectomy $+M R=$ Patients undergoing neurectomy combined with mesh removal; $\neq$ Various combinations are possible.

\section{Factors influencing outcome after remedial surgery}

Pain before surgery was very severe (median 7.5 on a $0-10$ scale, table 6.3). Following the operation, pain scores dropped significantly to $3.3(\mathrm{p}<0.01)$. Factors influencing remedial surgery outcome were gender (female, OR 0.30, 95\%CI 0.09-1.00), preoperative use of opioids (OR 0.38, 95\%CI 0.18-0.79), type of anaesthesia (spinal anaesthesia, OR 4.38, 95\%CI 2.23-9.00), intraoperative finding and removal of a meshoma (OR 4.66, $95 \% \mathrm{CI}$ 1.49-14.61) and removal of a histopathologically confirmed neuroma (OR 5.60, 95\%CI 1.19-26.50; table 6.4). Three variables were not included in this univariate analysis as $>30 \%$ of the data were missing (American Society of Anaesthesiologists (ASA) classification, $42 \%(n=57)$; type of hernia, $52 \%(n=70)$; funiculolysis during remedial surgery, $32 \%(n=43))$. 
TABLE 6.3:

Pain before and after surgery for Lichtenstein related inguinodynia

\begin{tabular}{lllll}
\hline & & \multicolumn{2}{l}{ Median values [IQR } & \\
& $\mathbf{n}(*)$ & Preoperative & Postoperative & P value \\
\hline NRS & & & & \\
Neurectomy & $68(11)$ & $7.0[6.0-8.0]$ & $5.0[1.0-7.0]$ & $<0.01$ \\
Neurectomy + limited mesh removal & $20(5)$ & $8.0[6.5-8.0]$ & $3.0[0.3-6.0]$ & $<0.01$ \\
Neurectomy + mesh removal & $37(0)$ & $8.0[6.8-8.5]$ & $1.0[0.0-5.3]$ & $<0.01$ \\
Mesh removal & $11(1)$ & $7.0[6.0-8.0]$ & $2.0[0.0-4.0]$ & $<0.01$ \\
Total & $136(17)$ & $7.5[6.0-8.0]$ & $3.3[0.0-6.9]$ & $<0.01$ \\
\hline
\end{tabular}

${ }^{*}$ Number of missing values; ${ }^{+} I Q R=$ Interquartile Range; ${ }^{\ddagger} N R S=$ Numerical Rating Scale.

TABLE 6.4:

Factors related to successful remedial pain surgery for inguinodynia after Lichtenstein repair (univariate analysis, $n=136$ )

95\% Confidence Interval

\begin{tabular}{|c|c|c|c|c|c|}
\hline Variable & $\mathbf{n}$ & Odds Ratio & Lower limit & Upper limit & P-value \\
\hline \multicolumn{6}{|c|}{ Patient related factors } \\
\hline $\mathrm{Age}^{\ddagger}(\mathrm{yr})$ & 136 & 1.01 & 0.99 & 1.04 & 0.36 \\
\hline Gender & 136 & & & & \\
\hline Male & 122 & 1 & Reference & Reference & Reference \\
\hline Female & 14 & 0.30 & 0.09 & 1.00 & $0.05^{*}$ \\
\hline $\mathrm{BMI}{ }^{\ddagger}\left(\mathrm{kg} / \mathrm{m}^{2}\right)$ & 130 & 1.00 & 0.91 & 1.11 & 0.98 \\
\hline Obesity & 130 & & & & \\
\hline $\mathrm{BMI} \geq 30\left(\mathrm{~kg} / \mathrm{m}^{2}\right)$ & 18 & 0.94 & 0.34 & 2.55 & 0.90 \\
\hline $\mathrm{BMI}<30$ & 112 & 1 & Reference & Reference & Reference \\
\hline Side & 136 & & & & \\
\hline Left & 51 & 1.17 & 0.58 & 2.36 & 0.66 \\
\hline Right & 85 & 1 & Reference & Reference & Reference \\
\hline Smoking status & 133 & & & & \\
\hline Non-smoker & 62 & 1 & Reference & Reference & Reference \\
\hline Active smoker & 46 & 0.58 & 0.27 & 1.25 & 0.17 \\
\hline Ex-smoker & 25 & 0.68 & 0.27 & 1.75 & 0.43 \\
\hline Work & 126 & & & & \\
\hline Light & 27 & 1 & Reference & Reference & Reference \\
\hline Moderate & 27 & 0.86 & 0.29 & 2.53 & 0.78 \\
\hline Heavy & 24 & 1.38 & 0.44 & 4.32 & 0.59 \\
\hline
\end{tabular}




\begin{tabular}{|c|c|c|c|c|c|}
\hline Retired & 18 & 1.38 & 0.40 & 4.78 & 0.62 \\
\hline Incapacitated & 24 & 0.41 & 0.13 & 1.27 & 0.12 \\
\hline Unemployed & 6 & 0.34 & 0.05 & 2.22 & 0.26 \\
\hline Time to reoperation ${ }^{\ddagger}$ & 136 & 1.00 & 0.99 & 1.01 & 0.80 \\
\hline NRS $\neq$ preoperative pain ${ }^{\ddagger}$ & 136 & 1.10 & 0.87 & 1.39 & 0.44 \\
\hline High preoperative pain score & 136 & & & & \\
\hline High preoperative $\mathrm{NRS}^{\S}(\geq 8)$ & 57 & 1.85 & 0.92 & 3.71 & 0.08 \\
\hline Preoperative $\mathrm{NRS}^{\S}<8$ & 79 & 1 & Reference & Reference & Reference \\
\hline Low preoperative pain score & 136 & & & & \\
\hline Low preoperative $\mathrm{NRS}^{\S}$ (i.e. $\leq 6$ ) & 37 & 0.98 & 0.46 & 2.09 & 0.96 \\
\hline Preoperative $\mathrm{NRS}^{\S}>6$ & 99 & 1 & Reference & Reference & Reference \\
\hline \multicolumn{6}{|l|}{ Relevant medical history } \\
\hline History of cardiovascular diseases & 132 & & & & \\
\hline Yes & 42 & 1.65 & 0.77 & 3.50 & 0.20 \\
\hline No & 90 & 1 & Reference & Reference & Reference \\
\hline History of pulmonary diseases & 132 & & & & \\
\hline Yes & 17 & 1.14 & 0.41 & 3.20 & 0.81 \\
\hline No & 115 & 1 & Reference & Reference & Reference \\
\hline History of prostatism in history & 128 & & & & \\
\hline Yes & 21 & 2.19 & 0.79 & 6.08 & 0.13 \\
\hline No & 107 & 1 & Reference & Reference & Reference \\
\hline History of anxiety or depression & 126 & & & & \\
\hline Yes & 12 & 0.73 & 0.22 & 2.39 & 0.60 \\
\hline No & 114 & 1 & Reference & Reference & Reference \\
\hline Injection successful & 125 & & & & \\
\hline Yes & 80 & 1.98 & 0.94 & 4.14 & 0.07 \\
\hline No & 45 & 1 & Reference & Reference & Reference \\
\hline Presence of dysejaculation & 107 & & & & \\
\hline Yes & 28 & 1.17 & 0.49 & 2.81 & 0.73 \\
\hline No & 79 & 1 & Reference & Reference & Reference \\
\hline Presence of testicular pain & 129 & & & & \\
\hline Yes & 33 & 0.65 & 0.29 & 1.44 & 0.29 \\
\hline No & 96 & 1 & Reference & Reference & Reference \\
\hline \multicolumn{6}{|l|}{ Preoperative use of analgesics } \\
\hline Prostaglandin use & 133 & & & & \\
\hline Yes & 62 & 1.12 & 0.56 & 2.21 & 0.75 \\
\hline No & 71 & 1 & Reference & Reference & Reference \\
\hline Non-opioid use & 134 & & & & \\
\hline Yes & 56 & 0.99 & 0.50 & 1.97 & 0.98 \\
\hline No & 78 & 1 & Reference & Reference & Reference \\
\hline Opioid use & 134 & & & & \\
\hline Yes & 45 & 0.38 & 0.18 & 0.79 & $<0.01^{* *}$ \\
\hline No & 89 & 1 & Reference & Reference & Reference \\
\hline
\end{tabular}




\begin{tabular}{|c|c|c|c|c|c|}
\hline \multicolumn{6}{|l|}{ Radiologic imaging } \\
\hline Ultrasound before remedial surgery & 129 & & & & \\
\hline Yes & 72 & 1.21 & 0.60 & 2.42 & 0.60 \\
\hline No & 57 & 1 & Reference & Reference & Reference \\
\hline CAT scan before remedial surgery & 130 & & & & \\
\hline Yes & 22 & 0.69 & 0.28 & 1.74 & 0.43 \\
\hline No & 108 & 1 & Reference & Reference & Reference \\
\hline MRI scan before remedial surgery & 129 & & & & \\
\hline Yes & 22 & 1.78 & 0.70 & 4.51 & 0.23 \\
\hline No & 107 & 1 & Reference & Reference & Reference \\
\hline \multicolumn{6}{|l|}{ Affected nerves preoperatively } \\
\hline Ilioinguinal nerve affected & 128 & & & & \\
\hline Yes & 29 & 0.81 & 0.35 & 1.86 & 0.62 \\
\hline No & 99 & 1 & Reference & Reference & Reference \\
\hline Iliohypogastric nerve affected & 128 & & & & \\
\hline Yes & 8 & 0.28 & 0.06 & 1.45 & 0.13 \\
\hline No & 120 & 1 & Reference & Reference & Reference \\
\hline Genitofemoral nerve affected & 127 & & & & \\
\hline Yes & 44 & 0.73 & 0.35 & 1.53 & 0.41 \\
\hline No & 83 & 1 & Reference & Reference & Reference \\
\hline Number of interventions ${ }^{\ddagger}$ & 136 & 0.68 & 0.45 & 1.02 & 0.06 \\
\hline History of recurrent hernias & 134 & & & & \\
\hline Yes & 30 & 0.76 & 0.34 & 1.72 & 0.51 \\
\hline No & 104 & 1 & Reference & Reference & Reference \\
\hline \multicolumn{6}{|c|}{ Surgery related factors } \\
\hline Longer operation time $^{\ddagger}$ & 136 & 1.01 & 1.00 & 1.03 & 0.09 \\
\hline Surgeon & 136 & & & & \\
\hline $\mathrm{RR}$ & 85 & 1 & Reference & Reference & Reference \\
\hline MS & 41 & 1.69 & 0.79 & 3.64 & 0.18 \\
\hline Other & 10 & 0.98 & 0.26 & 3.62 & 0.97 \\
\hline Anaesthesia & 136 & & & & \\
\hline General & 66 & 1 & Reference & Reference & Reference \\
\hline Spinal & 70 & 4.38 & 2.23 & 9.00 & $<0.01^{* *}$ \\
\hline \multicolumn{6}{|l|}{ Neurectomy } \\
\hline Ilioinguinal nerve & 134 & & & & \\
\hline Yes & 106 & 0.62 & 0.26 & 1.47 & 0.28 \\
\hline No & 28 & 1 & Reference & Reference & Reference \\
\hline Iliohypogastric nerve & 135 & & & & \\
\hline Yes & 32 & 0.56 & 0.25 & 1.24 & 0.15 \\
\hline No & 103 & 1 & Reference & Reference & Reference \\
\hline Genitofemoral nerve & 134 & & & & \\
\hline Yes & 40 & 1.14 & 0.54 & 2.41 & 0.73 \\
\hline No & 94 & 1 & Reference & Reference & Reference \\
\hline
\end{tabular}




\begin{tabular}{|c|c|c|c|c|c|}
\hline \multicolumn{6}{|l|}{ Perioperative identification } \\
\hline Ilioinguinal nerve & 133 & & & & \\
\hline Yes & 106 & 0.97 & 0.41 & 2.26 & 0.94 \\
\hline No & 27 & 1 & Reference & Reference & Reference \\
\hline Iliohypogastric nerve & 135 & & & & \\
\hline Yes & 35 & 0.71 & 0.33 & 1.54 & 0.39 \\
\hline No & 100 & 1 & Reference & Reference & Reference \\
\hline Genitofemoral nerve & 135 & & & & \\
\hline Yes & 46 & 1.92 & 0.92 & 4.00 & 0.08 \\
\hline No & 89 & 1 & Reference & Reference & Reference \\
\hline Presence and removal of a meshoma ${ }^{\dagger}$ & 132 & & & & \\
\hline Yes & 23 & 4.66 & 1.49 & 14.61 & $<0.01^{* *}$ \\
\hline No & 109 & 1 & Reference & Reference & Reference \\
\hline Presence and removal of a neuroma & 120 & & & & \\
\hline Yes & 13 & 5.60 & 1.19 & 26.50 & $0.03^{*}$ \\
\hline No & 107 & 1 & Reference & Reference & Reference \\
\hline
\end{tabular}

Variables marked in gray demonstrate a significant association with persistent groin pain after remedial surgery (univariate binary logistic regression analysis). Odds ratio $>1.0$ indicates a higher chance on a successful outcome after operation. Odds ratio $<1.0$ indicates a lower chance on a successful operation. ${ }^{*} p \leq 0.05 ;{ }^{* *} p \leq 0.01 ;{ }^{*}$ Meshoma $=$ folding and wrinkling of the inserted mesh, varying from a mass-like density to more subtle effects of mesh wrinkling or fibrosis ${ }^{19,20}$, as determined by the operating surgeon; ${ }^{*}$ continuous variable; ${ }^{5}$ NRS $=$ Numerical Rating Score.

The number of operations included in the multivariate analysis was 116 . When predicting factors were added to this analysis, three parameters greatly determined surgical efficacy. Preoperative use of opioids (OR 0.37, 95\%CI 0.16-0.87) negatively influenced the operative results. Conversely, remedial surgery under spinal anaesthesia (OR 4.04, 95\%CI 1.76-9.23) and removal of a meshoma (OR 5.31, 95\%CI 1.48-19.05) were both associated with a favourable outcome. The positive predictive value of the model including these three variables was $61 \%$ with an overall correct percentage of $66 \%$.

\section{Pain recall bias}

Pairs of pain scores (prospectively obtained from the electronic patient file and retrospectively determined during the telephone interview) were available in 85 patients (56\%). Interestingly, prospectively scored pain was consistently lower compared to retrospectively obtained scores (mean $7.2 \pm 1.5$ vs. $7.8 \pm 1.3, \mathrm{p}<0.01$ ) indicating the presence of a recall effect, albeit small. Stratified analysis of patients failing remedial surgery (i.e. pain reduction $<50 \%$ ) with a complete set of pain scores also demonstrated that prospectively obtained pain scores were lower $(\mathrm{p}<0.01)$ compared to retrospective values $(n=43,7.1 \pm 1.4$ versus $7.7 \pm 1.2)$. A similar phenomenon was found in the group 
with successful outcomes $(\mathrm{n}=42,7.3 \pm 1.6$ versus $7.8 \pm 1.4, \mathrm{p}=0.03)$. Therefore, a small recall error systematically affected both the failed group and the successful group in a similar way.

\section{Observer bias}

Baseline characteristics of populations harbouring patients with an incomplete set of pain data ('missing', $\mathrm{n}=17$ ) and a complete set of data ('complete', $\mathrm{n}=136$ ) were compared for the potential presence of observer bias. Table 6.5 illustrates that both populations were not different with reference to gender, age, body mass index (BMI) and levels of preoperative pain.

\section{TABLE 6.5:}

Baseline characteristics of groups with missing and complete sets of pain scores. Data are presented as means \pm standard deviation or median [interquartile range]

\begin{tabular}{lllll}
\hline Baseline Characteristic & Missing $(\mathbf{n}=\mathbf{1 7})$ & Complete $(\mathbf{n}=\mathbf{1 3 6})$ & $\mathbf{p}$-value & Test \\
\hline Sex ratio (M/F) & $16 / 1$ & $122 / 14$ & 0.56 & $X^{2}$ test \\
Age (years) & $58 \pm 14$ & $54 \pm 13$ & 0.27 & Unpaired t-test \\
Body Mass Index $\left(\mathrm{kg} / \mathrm{m}^{2}\right)$ & $26[22-29]$ & $26[24-29]$ & 0.38 & Mann-Whitney U test \\
Preoperative pain score & $7.4 \pm 1.7$ & $7.3 \pm 1.4$ & 0.81 & Unpaired t-test \\
\hline
\end{tabular}

\section{DISCUSSION}

Evidence based recommendations for the institution of remedial groin surgery in patients suffering from inguinodynia following Lichtenstein hernia repair are currently lacking. The present study is the first to aim at identifying factors predicting outcome after this specific type of groin pain surgery. The results demonstrate that the intraoperative finding and removal of a meshoma favourably (OR 5.31) influences outcome. Moreover, the study strongly supports the use of perioperative spinal anaesthesia (OR 4.04) as a tool to increase the chance on postoperative success. Conversely, preoperative use of opioids (OR 0.37) is associated with a limited chance on a successful outcome. Consequently, consulting individuals with inguinodynia on these issues may lead to improved patient selection and optimized outcome. 
An important factor determining success after remedial surgery is the removal of a meshoma. In the present study, a meshoma was defined by the operating surgeon as suggested by a recently published international consensus algorithm for the management of postsurgical inguinal pain ${ }^{19}$. Different gradations of a meshoma may be present, varying from a mass-like density to more subtle effects of mesh wrinkling or fibrosis ${ }^{19}$. This may lead to pain by a volume effect or by mechanical pressure ${ }^{14}$. Literature on this phenomenon is scarce, and early interventions do not prevent the mesh from creasing. Nonetheless, careful patient history taking and physical examination may be helpful to hypothesize the presence of a meshoma prior to surgery. For instance, patients may report a sensation of tightness or 'a foreign body feeling. A meshoma may also be palpable in non-obese individuals ${ }^{14}$. Radiographic imaging including ultrasonography or magnetic resonance imaging can contribute to the diagnosis ${ }^{19,}{ }^{20}$. Previous studies have also suggested that mesh removal is effective in reducing groin pain after Lichtenstein repair ${ }^{9}$ 13, 14, 19, 21. The present study also suggests that, if a meshoma is indeed present, its removal reduces the risk on persistent groin pain.

The relevance of type of anaesthetic technique in relation to postoperative success raises questions. To date, results of studies in primary inguinal hernia repair focussing on this issue were not consistent. For instance, one study found no influence of type of technique ${ }^{22}$ whereas two other studies favoured spinal anaesthesia compared to general anaesthesia regarding the issue of persistent postoperative pain ${ }^{23,24}$. The present data set identified a significant soothing effect of a spinal type of anaesthesia on inguinodynia. Spinal anaesthesia induces peripheral nerve blocks and prevents the transmission of nociceptive noxious impulses secondary to peripheral tissue damage via C-fibres, from the operative site to the spinal $\operatorname{cord}^{25,26}$. Prolonged impulse stimulation along unmyelinated C-fibres afferents may cause transneuronal morphologic changes in the spinal cord resulting in long-lasting spinal cord hyperexcitability ${ }^{25,27,28}$. Conversely, blocking of nociceptive signals by a spinal technique prevents the initial transfer of pain stimuli from the affected nerve towards the central nervous system and possibly averts spinal hyperexcitability and consequent chronic pain. This finding clearly needs future research in a randomized setting, especially in chronic pain patients undergoing surgery for pain in the groin and lower extremities.

The preoperative use of opioids was significantly associated with a less favourable outcome. Previous pain treatment as an important determinant of poor outcome 
following remedial surgery was described earlier ${ }^{6}$. Current literature on opioids as a treatment for chronic neuropathic pain recommends these medications as a first-line treatment option in certain clinical circumstances ${ }^{29}$. However, these recommendations are based on trials of a maximum of ten weeks of duration ${ }^{29,30}$. In contrast, median pain duration in the present study was 32 months. Therefore, data on the efficacy or adverse effects of opioids possibly do not apply to a population with chronic inguinodynia ${ }^{30}$. One may speculate that the negative effect of long-lasting opioid therapy in our population is due to adverse phenomena such as paradoxical hyperalgesia. If present, patients may paradoxically become more sensitive to pain as a result of ongoing opioid use $\mathrm{e}^{31}$. This potential complication of opioids is often overlooked ${ }^{31}$ and may explain some findings of the present study.

The univariate analysis demonstrated that removal of a neuroma was associated with a beneficial outcome. Previous studies have also reported pain relief after neuroma removal in up to $80 \%$ of patients $^{32}$. The incidence of neuromas in patients with chronic pain after hernia repair has not been investigated properly. Histopathological examination of a potential neuroma is mandatory for verification. In one study, neuroma formation was found in 6 of 49 patients $(12 \%)^{10}$. A second report mentioned the formation of a histopathologically confirmed neuroma in $14 \%$ of the population ${ }^{12}$. A third study reported that approximately $13 \%$ of patients with mesh related pain syndromes had developed a neuroma after primary hernia repair ${ }^{14}$. These remarkably similar percentages suggest that pain due to a neuroma is found in about one of seven patients undergoing remedial surgery for persistent groin pain following a Lichtenstein repair.

An assumed confounding factor in pain outcome after primary hernia repair is gender as females sustain a higher risk of developing postoperative pain ${ }^{33-36}$. Conversely, several other studies suggested no significant association between female and persistent postoperative pain ${ }^{17,37,38}$. Our data demonstrate that groin pain surgery is less successful in females in the univariate analysis. However, when correction for potential confounding factors is performed, this association is annulled. This suggests that other risk factors have confounded the association of female and persistent groin pain. Other risk factors suggested by previous reports (table 6.1) were not identified in the present study. It may well be that most differences are due to the fact that these studies were performed in populations after primary inguinal repair. 
Success after remedial surgery was achieved in $54 \%$ of the present patients. This relatively low success rate after one surgical attempt is due to the fact that our approach is 'tailored' as opposed to a standardized triple neurectomy ${ }^{6,7,10}$. Some initially unsuccessful patients became pain free after a second surgical intervention. However, these secondary operations were excluded from this study for reasons as previously described.

The current study is retrospective and therefore subject to limitations. Observer bias may potentially be a threat to its internal validity. Missing data associated with its retrospective character may lead to biased results. Therefore, baseline characteristics of groups with complete and incomplete data sets were compared to study the presence of information bias (table 6.5). This analysis demonstrated similar group characteristics indicating absence of observer bias. Another possible flaw is the subjective selection of variables that was guided by the available literature on inguinodynia after primary Lichtenstein repair. Important other confounders may have been missed. An error related to recalled pain that was responsible for consistently higher preoperative pain levels (NRS, 0.6 on a $0-10$ scale) was similar in magnitude in populations after failed and successful surgery. However, recall effects are only thought influential when values are different for patients with failed groin pain surgery ('cases') compared to successfully operated patients ('controls') ${ }^{39}$. Non-differential recall errors result in an OR closer to zero. Therefore it is unlikely that these errors account for any false associations ${ }^{40}$. Consequently, no differential misclassification occurred in the present study.

In conclusion, the present study reported on factors predicting success after remedial surgery in patients with inguinodynia following a previous Lichtenstein hernia repair. If a meshoma is found, removal increases the chances of success. The preoperative use of opioids negatively influenced surgical outcome. Furthermore, patients who underwent surgery using spinal anaesthesia reported less long-term postoperative pain compared to patients after general anaesthesia. Findings of the present study have prompted us to initiate a randomized controlled trial on the effect of anaesthetic technique for remedial surgery in patients with inguinodynia.

\section{Acknowledgments}

We would like to thank dr. J.P. Dieleman (epidemiologist) for her contribution to the statistical analyses performed in this study. 


\section{REFERENCE LIST}

1. Nienhuijs S, Staal E, Strobbe L, et al. Chronic pain after mesh repair of inguinal hernia: a systematic review. Am J Surg 2007;194(3):394-400.

2. Vuilleumier H, Hubner M, Demartines N. Neuropathy after herniorrhaphy: indication for surgical treatment and outcome. World J Surg 2009;33(4):841-5.

3. Ferzli GS, Edwards E, Al-Khoury G, et al. Postherniorrhaphy groin pain and how to avoid it. Surgical Clin North Am 2008;88(1):203-16, x-xi.

4. O’Dwyer PJ, Alani A, McConnachie A. Groin hernia repair: postherniorrhaphy pain. World J Surg 2005;29(8):1062-5.

5. Wijsmuller AR, Lange JF, van Geldere D, et al. Surgical techniques preventing chronic pain after Lichtenstein hernia repair: state-of-the-art vs daily practice in the Netherlands. Hernia 2007;11(2):147-51.

6. Loos MJ, Scheltinga MR, Roumen RM. Tailored neurectomy for treatment of postherniorrhaphy inguinal neuralgia. Surgery 2010;147(2):275-81.

7. Amid PK. Causes, prevention, and surgical treatment of postherniorrhaphy neuropathic inguinodynia: triple neurectomy with proximal end implantation. Hernia 2004;8(4):343-9.

8. Kim DH, Murovic JA, Tiel RL, et al. Surgical management of 33 ilioinguinal and iliohypogastric neuralgias at Louisiana State University Health Sciences Center. Neurosurgery 2005;56(5):1013-20; discussion 13-20.

9. Aasvang EK, Kehlet $\mathrm{H}$. The effect of mesh removal and selective neurectomy on persistent postherniotomy pain. Ann Surg 2009;249(2):327-34.

10. Amid PK. A 1-stage surgical treatment for postherniorrhaphy neuropathic pain: triple neurectomy and proximal end implantation without mobilization of the cord. Arch Surg 2002;137(1):100-4.

11. Chen DC, Hiatt JR, Amid PK. Operative management of refractory neuropathic inguinodynia by a laparoscopic retroperitoneal approach. JAMA Surgery 2013;148(10):962-67.

12. Bischoff JM, Enghuus C, Werner MU, et al. Long-term follow-up after mesh removal and selective neurectomy for persistent inguinal postherniorrhaphy pain. Hernia 2013;17(3):33945.

13. Heise CP, Starling JR. Mesh inguinodynia: a new clinical syndrome after inguinal herniorrhaphy? J Am Coll Surg 1998;187(5):514-8. 
14. Zwaans WA, Perquin CW, Roumen RM, et al. Mesh removal and selective neurectomy for persistent groin pain following Lichtenstein repair. World J Surg 2017; 41(3):701-12

15. Aasvang EK, Gmaehle E, Hansen JB, et al. Predictive risk factors for persistent postherniotomy pain. Anesthesiology 2010;112(4):957-69.

16. Campanelli G, Bertocchi V, Cavalli M, et al. Surgical treatment of chronic pain after inguinal hernia repair. Hernia 2013;17(3):347-53.

17. Franneby U, Sandblom G, Nordin P, et al. Risk factors for long-term pain after hernia surgery. Ann Surg 2006;244(2):212-9.

18. van Assen T, Boelens OB, van Eerten PV, et al. Surgical Options after a Failed Neurectomy in Anterior Cutaneous Nerve Entrapment Syndrome. World J Surg 2014.

19. Lange JF, Kaufmann R, Wijsmuller AR, et al. An international consensus algorithm for management of chronic postoperative inguinal pain. Hernia 2014.

20. Amid PK. Radiologic images of meshoma: a new phenomenon causing chronic pain after prosthetic repair of abdominal wall hernias. Arch Surg 2004;139(12):1297-8.

21. Keller JE, Stefanidis D, Dolce CJ, et al. Combined open and laparoscopic approach to chronic pain after inguinal hernia repair. Am Surg 2008;74(8):695-700; discussion 00-1.

22. Bay-Nielsen M, Perkins FM, Kehlet H. Pain and functional impairment 1 year after inguinal herniorrhaphy: A nationwide questionnaire study. Ann Surg 2001;233(1):1-7.

23. Steegers MA, Wilder-Smith OH. [Late chronic pain after surgery is prevented with good perioperative analgesics]. NTVG 2009;153(12):562-6.

24. Ozgun H, Kurt MN, Kurt I, et al. Comparison of local, spinal, and general anaesthesia for inguinal herniorrhaphy. Eur J Surg 2002;168(8-9):455-9.

25. Tverskoy M, Cozacov C, Ayache M, et al. Postoperative pain after inguinal herniorrhaphy with different types of anaesthesia. Anesth Analg 1990;70(1):29-35.

26. Kitahata LM. Pain pathways and transmission. Yale J Biol Med 1993;66(5):437-42.

27. Woolf CJ. Evidence for a central component of post-injury pain hypersensitivity. Nature 1983;306(5944):686-8.

28. Sugimoto T, Takemura M, Sakai A, et al. Rapid transneuronal destruction following peripheral nerve transection in the medullary dorsal horn is enhanced by strychnine, picrotoxin and bicuculline. Pain 1987;30(3):385-93.

29. Dworkin RH, O'Connor AB, Audette J, et al. Recommendations for the pharmacological management of neuropathic pain: an overview and literature update. Mayo Clinic Proceedings 2010;85(3 Suppl):S3-14. 
30. Eisenberg E, McNicol E, Carr DB. Opioids for neuropathic pain. Cochrane Database Syst Rev 2006(3):CD006146.

31. Lee SH, Cho SY, Lee HG, et al. Tramadol induced paradoxical hyperalgesia. Pain physician 2013;16(1):41-4.

32. Ducic I, Dellon AL. Testicular pain after inguinal hernia repair: An approach to resection of the genital branch of genitofemoral nerve. J Am Coll Surg 2004;198(2):181-84.

33. Aasvang E, Kehlet H. Chronic postoperative pain: the case of inguinal herniorrhaphy. Br J Anaesth 2005;95(1):69-76.

34. Kalliomaki ML, Meyerson J, Gunnarsson U, et al. Long-term pain after inguinal hernia repair in a population-based cohort; risk factors and interference with daily activities. Eur J Pain 2008;12(2):214-25.

35. Bischoff JM. Management of Persistent Inguinal Postherniorrhaphy Pain: Pharmacological and Surgical Aspects. University of Copenhagen, 2014.

36. Sondenaa K, Nesvik I, Breivik K, et al. Long-term follow-up of 1059 consecutive primary and recurrent inguinal hernias in a teaching hospital. Eur J Surg 2001;167(2):125-9.

37. Massaron S, Bona S, Fumagalli U, et al. Analysis of post-surgical pain after inguinal hernia repair: a prospective study of 1,440 operations. Hernia 2007;11(6):517-25.

38. Liem MS, van Duyn EB, van der Graaf Y, et al. Recurrences after conventional anterior and laparoscopic inguinal hernia repair: a randomized comparison. Ann Surg 2003;237(1):136-41.

39. Sedgwick P. What is recall bias? BMJ 2012;344.

40. Delgado-Rodriguez M, Llorca J. Bias. J Epidemiol Community Health 2004;58(8):635-41. 



\section{CHAPTER 7}

Chronic postoperative groin pain requiring remedial surgery: spinal or general anaesthesia?

W.A.R. Zwaans

S.M. van Kuijk

L.H.P.M. le Mair

M. van Kleef

M.R.M. Scheltinga

R.M.H. Roumen

Under review 


\section{ABSTRACT}

Objectives: Conservative treatments for chronic pain following open inguinal hernia repair are often to no avail. Remedial surgery such as a neurectomy may be considered but success rates are suboptimal. Type of anaesthesia may influence outcome. Aim of the present study was to investigate whether remedial surgery for inguinodynia is more successful if performed under spinal anaesthesia or general anaesthesia.

Design: Patients who underwent open remedial surgery for a groin pain syndrome between 2000 and 2014 in a single were identified by a database search. Evidencebased confounding patient characteristics and specifics of surgery were extracted from the hospital's electronic information system. An univariate binary logistic regression analysis identified factors possibly predicting treatment outcome. Significant variables $(\mathrm{p} \leq 0.1)$ were included in a bivariate and subsequent multivariate logistic regression analysis to correct for potential confounders. Success was defined as $\geq 50 \%$ decrease in pain level

Results: A total of in 339 patients (63\% males, median age 50, range 18-88) undergoing surgery for groin pain were eligible for study. Surgery was performed under spinal anaesthesia in $41 \%$. Overall success rate was $66 \%$. After correction for confounders in the multivariate analysis, spinal anaesthesia showed a significant positive association with a successful outcome (OR 1.9, 95\%CI 1.1-3.4). This effect was most evident in patients who underwent a neurectomy (OR 2.3, 95\% CI 1.3-4.1).

Conclusions: Remedial surgery for chronic postoperative groin pain syndromes may be twice as successful if the procedure is performed under spinal anaesthesia. This effect is most evident if a neurectomy is involved. Effect size is determined by statistical methods and therefore findings should be interpreted with caution. A prospective randomized trial circumventing these types of bias is underway. 


\section{INTRODUCTION}

Persistent inguinodynia (groin pain) is currently the most challenging complication following inguinal hernia surgery replacing the former issue of a hernia recurrence ${ }^{1}$. Postherniorrhaphy groin pain is associated with substantial morbidity, prolonged hospital stay and increased health care costs ${ }^{2,3}$. Persistent groin pain may also occur after a caesarean incision (post-Pfannenstiel pain syndrome) ${ }^{4}$, endometriosis of the uterus' round ligament ${ }^{5}$ or following urologic operations ${ }^{6}$. If conservative treatments for all of these groin pain syndromes have failed, a surgical exploration including a neurectomy may be beneficial ${ }^{4,7}$.

In general, effectiveness of remedial surgery for inguinodynia of either origin varies considerably and optimization of success rates is desirable. It must be appreciated that current literature on surgical strategies aimed at postoperative pain reduction is primarily based on studies involving postherniorrhaphy pain $^{1,2,8}$. A considerable number of studies in primary inguinal hernia repair concluded that spinal anaesthesia, when compared to general anaesthesia, was superior in terms of postoperative pain relief ${ }^{9}{ }^{10}$. In addition, a recent study on potential risk factors determining outcome of remedial groin pain surgery also found that a regional anaesthetic technique was associated with successful outcome $^{11}$. However, this beneficial effect of spinal anaesthesia was only investigated for inguinodynia after a Lichtenstein hernia repair. Whether this type of anaesthesia is also beneficial in remedial pain surgery for other chronic groin pain syndromes is uncertain. This study aimed at investigating whether spinal anaesthesia is preferable over general anaesthesia in patients undergoing remedial surgery for various types of chronic inguinodynia. 


\section{METHODS}

\section{Protocol and ethics approval}

This present paper was designed and written according to the guidelines of the STROBE statement for reporting observational studies ${ }^{12}$. No permission from the medical ethics committee of the hospital was necessary since it involved evaluation of previously collected data and auditing of own results.

\section{Setting}

This retrospective cohort study was performed in a separate surgical outpatient department of Máxima Medical Centre (MMC), termed the 'SolviMáx Center of Excellence for Abdominal Wall and Groin Pain'. In the year of 2015, a total of 1060 new patients were analyzed for chronic abdominal wall or groin pain.

\section{Diagnostic and preoperative criteria}

At SolviMáx, each patient is evaluated using a standardized history case report form and physical examination. If entrapment of an inguinal nerve (ilioinguinal, iliohypogastric or genital branch of the genitofemoral nerve) is considered, a 5-10 $\mathrm{ml} \mathrm{1-2 \%} \mathrm{lidocaine}$ diagnostic injection at the maximal pain point is proposed. If long-term pain relief (beyond 2 weeks) after 1-3 injection(s) is not achieved, or pain is deemed mesh-related, specifics of a surgical therapy are communicated. All patients consented verbally and in writing. Surgery is always performed as an open procedure under a general or a spinal anaesthetic technique in a day care setting. However, choice of type of anaesthesia is left to the discretion of the patient, the anaesthesiologist or the surgeon within a shared subjective decision process.

\section{Study design}

The present study was performed between April and July, 2015. Identification of study patients was performed by a database search with the use of an exclusive SolviMáx operative code that is standard assigned to each surgical procedure including a neurectomy and/ or meshectomy. All consecutive patients who underwent open remedial surgery between 2000 to 2014 were extracted from the hospital's electronic information system. Inclusion criteria were $\geq 18$ years of age, suffering from chronic ( $\geq 3$ months) inguinodynia and open 
remedial surgery for groin pain by either a neurectomy, a meshectomy or a combination of these. A portion of the patients was previously studied ${ }^{11}$.

Excluded were patients with pain due to intercostal nerve entrapment (abdominal cutaneous nerve entrapment syndrome, ACNES) or the lateral femoral cutaneous nerve (meralgia paresthetica). Patients were also excluded if operative reports were missing, if they had undergone a retroperitoneal neurectomy (general anaesthesia required), laparoscopic remedial surgery, were cognitively impaired or had groin infections or malignancy at the time of surgery. Lastly, patients who underwent multiple remedial operations or bilateral surgery were also excluded to avoid multiple inclusions of patients' determinants in the regression analyses. Patients having contra-indications for general anaesthesia (body mass index (BMI) $\geq 40 \mathrm{~kg} \mathrm{~m}^{-2}$, symptomatic hiatus hernia or severe gastroesophageal reflux disease) or spinal anaesthesia (coagulation disorders, infection located near puncture site, pre-existent neurological disorders, disability to understand Dutch language) as judged by the anaesthesiologist were excluded.

\section{Confounding variables}

Potential evidence-based confounders such as age, gender, BMI, smoking status, index surgery, time after index surgery, preoperative use of opioids, side of pain, preferred anaesthetic technique as well as surgery-specific determinants including type of anaesthesia, operation time, type of remedial surgery, intraoperative identification of nerves and adverse effects were all collected. All data were extracted and separately entered in a database after which patient records were anonymized.

\section{Outcome measures}

Primary outcome measure was treatment success as documented in the electronic file. Surgery was termed successful if a $\geq 50 \%$ decrease in pain level compared to preoperative verbal response pain scores (Visual Analogue Scale, VAS or Numerical Rating Scale, NRS) was reported by the patient. This outpatient evaluation was obtained 6-8 weeks postoperatively. Secondary outcomes were complications following remedial surgery and associations between patient characteristics and surgery-specific determinants and outcome. Moreover, the relation between anaesthetic technique and types of remedial surgery (neurectomy, meshectomy or a combination of these) in terms of success was also analyzed. 


\section{Anaesthetic techniques during remedial surgery}

All patients received paracetamol and naproxen as oral premedication, unless contraindications were present. Patients were continuously monitored with electrocardiograms and pulse oximetry. Preload of $500 \mathrm{ml} \mathrm{NaCl} \mathrm{0,9 \%} \mathrm{was} \mathrm{given} \mathrm{before} \mathrm{induction} \mathrm{of}$ anaesthesia. Arterial blood pressure was recorded non-invasively every 5 minutes during surgery and every 15 minutes in the recovery unit. All operations were performed with patients in the supine position.

\section{Spinal anaesthesia}

If requested and decided upon during the preoperative screening, midazolam was given intravenously in a dose depending on age, BMI and medical history. The patient was placed in the upright position. A subarachnoid puncture via the midline allowed admission to the L3-4 or L2-3 intervertebral interspace using a 25-G or 27-G pencil point needle through an introducer (Braun - Pencan ${ }^{\circledR}$ ) followed by a $75 \mathrm{mg}$ dose of $5 \%$ hyperbaric articaine. The patient lied down immediately after the spinal injection. No additional analgesics were given during the operation.

\section{General anaesthesia}

A laryngeal mask was used (LMA, Laryngeal Mask Company - Unique ${ }^{\mathrm{Tn}}$ ). After preoxygenation, induction was achieved with intravenous propofol 2-3 mg kg-1 and sufentanil 0.2-0.3 mcg kg-1. The LMA was inserted and anaesthesia was maintained with sevoflurane (1.5-1.8 minimum alveolar concentration) in an air-oxygen mixture via a circle anaesthesia breathing system. The patient was allowed to breathe spontaneously. Sufentanil was titrated according to requirement. All patients received granisetron $1 \mathrm{mg}$ intravenously (iv) as an antiemetic after induction of anaesthesia and a single dose of 0.1 $\mathrm{mg} \mathrm{kg}^{-1}$ morphine iv during the procedure. The majority of the patients also received an additional single dose of $0.25 \mathrm{mg} \mathrm{kg}^{-1}$ esketamine iv.

\section{Statistical analysis}

IBM SPSS Statistics version 22.0 was used for statistical analysis. Values were expressed as mean \pm standard deviation (SD) or median [interquartile range (IQR)] in normally distributed and non-normally distributed data, respectively. Logistic regression was used to estimate the association between type of anaesthesia and successful outcome, corrected 
for confounding variables. In the primary analysis, confounders were selected based on a statistically significant association with treatment outcome in a univariate regression. Subsequent multivariable logistic regression containing both the potential confounder and type of anaesthesia was used to test whether the variable confounded the association between type of anaesthesia and successful outcome. Subsequent multivariable regression with all confounders selected in the previous step was performed by using the likelihood ratio backward stepwise elimination. OR and corresponding 95\%-confidence intervals (95\%CI) were calculated. The significance level of the backward stepwise elimination was set at $\mathrm{p} \leq 0.1$. As a sensitivity analysis, the OR for the association of spinal anaesthesia with successful outcome was calculated by multivariable analysis with correction for all possible confounders, in contrast to using only those that were significant. The calculation of this 'fully adjusted OR' was performed to test whether results were robust to using other equal statistical approaches.

To determine the effect of anaesthesia on treatment outcome in the three different types of remedial surgeries (neurectomy, meshectomy or combined), a stratified analysis of these subgroups was performed by univariate logistic regression using anaesthesia as independent factor. Stratification by primary surgery or aetiology was also performed to evaluate the effect of anaesthesia on outcome in different diagnosis groups. Only stratification of aetiological factors with more than 20 patients per diagnosis group were considered.

In general, patients who prefer spinal anaesthesia may have a priori reduced probability of persistent postoperative pain, as anxiety is known to influence levels of postoperative pain $^{13}$. Therefore, the preferred anaesthetic technique may confound results of the present study. To evaluate the decisive role of a patient on type of anaesthesia, the correlation of the preferred anaesthetic technique and applied technique was calculated using the nonparametric Spearman's rank correlation coefficient. Patients failing spinal anaesthesia who subsequently received general anaesthesia were classified as 'general anaesthesia', leading to dichotomization of the outcome measure. The significance level ( $p$-value) for these results was set $\leq 0.05$. 


\section{RESULTS}

\section{Participants}

A total of 1,779 remedial operations were performed between 2000-2014. Following exclusion of surgery in non-groin areas $(n=993)$ and other reasons $(n=441), 345$ patients undergoing remedial surgery for groin pain were eligible for analysis. As follow-up was lacking in six patients, 339 surgical procedures were analyzed (figure 7.1).

\section{Characteristics of study population}

Most patients had a history of pain following earlier open inguinal hernia repair $(n=225,66 \%)$. Other operations responsible for inguinodynia included gynaecologic, urologic and plastic procedures (figure 7.1). Index operations were performed between 1967-2014. Mean age was 50 years (range 18-88) and 63\% was male. Mean pain intensity prior to operation was $7.1 \pm 1.5$ on the $0-10$ NRS scale. The ratio of general anaesthesia to spinal anaesthesia was 59 to 41 (table 7.1).

\section{Primary outcome}

Success as judged by the patient at the 6-8 week follow-up outpatient evaluation was $66 \%(n=223)$. Characteristics of successful and unsuccessful patients are listed in table 7.1. Female gender, young age ( $<30$ years), index operation other than hernia repair, longer duration of pain and preoperative use of opioids were associated with an unsuccessful outcome (table 7.2). In contrast, surgery-related variables including use of spinal anaesthesia, identification of the genital branch of genitofemoral nerve and removal of a meshoma or a neuroma were related to a beneficial outcome. The bivariable logistic regression demonstrated similar results as the univariate analysis, excluding the index operation and finding and removal of a neuroma. These variables showed no confounding factors regarding anaesthesia and outcome as based on the present data and were therefore removed from the multivariable analysis. 


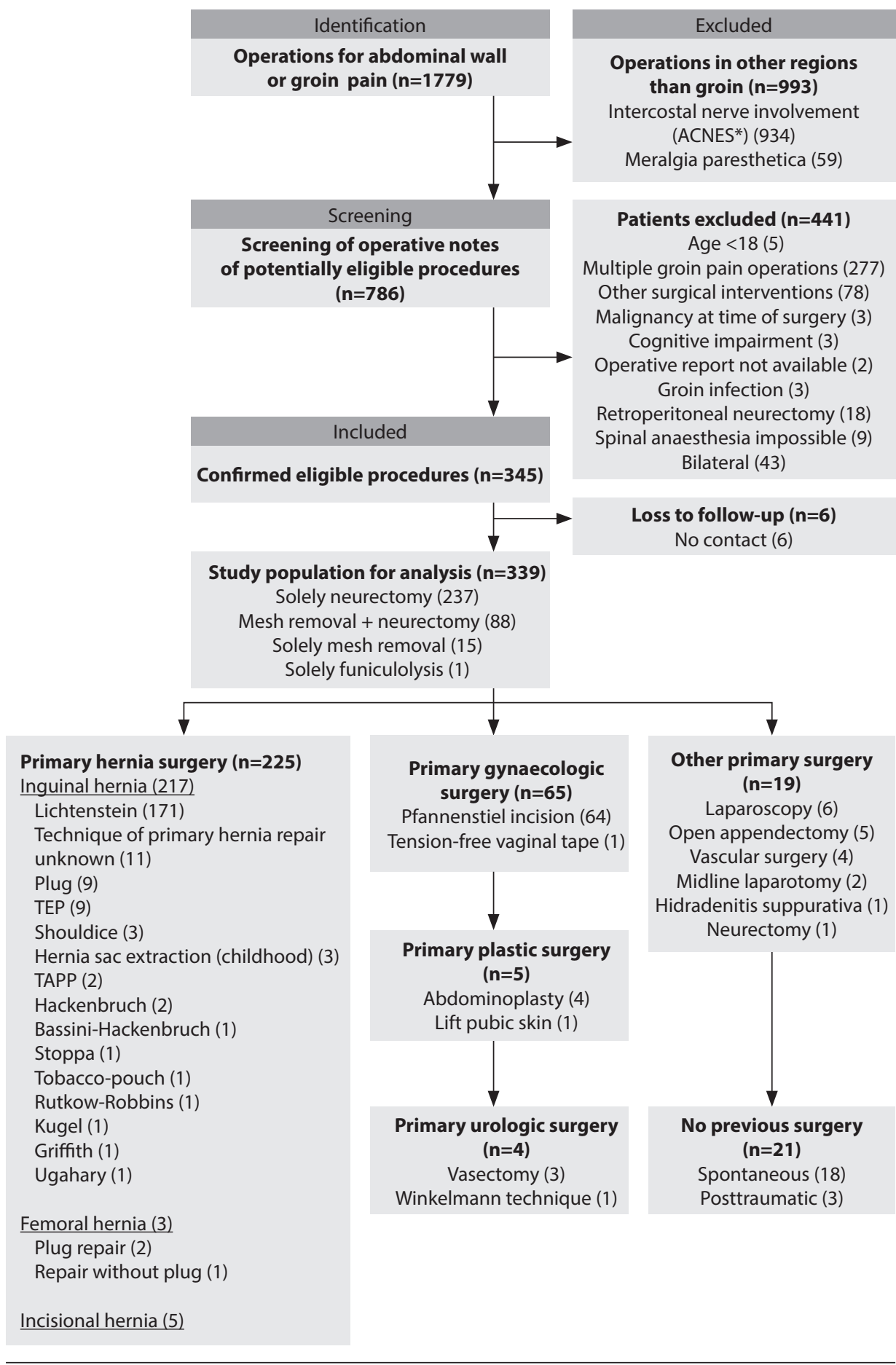

FIGURE 7.1:

Flowchart diagram of selection of operations. ${ }^{*}$ ACNES $=$ Abdominal Cutaneous Nerve Entrapment Syndrome. 
TABLE 7.1:

Baseline characteristics of patients undergoing remedial surgery for persistent groin pain syndromes

\begin{tabular}{|c|c|c|c|}
\hline Patient characteristics & Total group & Successes & Failures \\
\hline Age & 50.5 [range 18-88] & 51.7 [range 18-88] & 48.3 [range 20-82] \\
\hline \multirow[t]{2}{*}{ Sex } & Male 215 (63\%) & Male $155(70 \%)$ & Male $60(52 \%)$ \\
\hline & Female 124 (37\%) & Female 68 (30\%) & Female 56 (48\%) \\
\hline Pain intensity (NRS*) & $7.1 \pm 1.5$ & $7.1 \pm 1.5$ & $7.2 \pm 1.4$ \\
\hline Duration of pain (mo) & 38 [18-88] & $36[16-88]$ & 44 [19-94] \\
\hline Neuropathic component & $95.4 \%$ & $94.2 \%$ & $97.4 \%$ \\
\hline \multirow[t]{2}{*}{ Side surgery } & Left 140 (41\%) & Left 96 (43\%) & Left 44 (38\%) \\
\hline & Right 199 (59\%) & Right 127 (57\%) & Right 72 (62\%) \\
\hline \multirow[t]{2}{*}{ Type of anaesthesia } & General 201 (59\%) & General 117 (52\%) & General 84 (72\%) \\
\hline & Spinal $138(41 \%)$ & Spinal 106 (48\%) & Spinal $32(28 \%)$ \\
\hline \multirow{5}{*}{$\begin{array}{l}\text { Preferred anaesthetic } \\
\text { technique }\end{array}$} & General $73(22 \%)$ & General 43 (19\%) & General 30 (26\%) \\
\hline & Spinal $42(12 \%)$ & Spinal 31 (14\%) & Spinal 11 (10\%) \\
\hline & Local 6 (2\%) & Local $3(1 \%)$ & Local 3 (3\%) \\
\hline & None 7 (2\%) & None $4(2 \%)$ & None $3(3 \%)$ \\
\hline & Missing 211 (62\%) & Missing 142 (64\%) & Missing 69 (60\%) \\
\hline Operation time & $36.0[25-52]$ & $40[26-57]$ & $32.0[24-45]$ \\
\hline Body Mass Index $\left(\mathrm{kg} \mathrm{m}^{-2}\right)$ & $26.2 \pm 3.9$ & $26.3 \pm 3.7$ & $26.0 \pm 4.5$ \\
\hline \multirow[t]{4}{*}{ Smoking status } & $\begin{array}{l}\text { Non-smoker } 107 \\
(32 \%)\end{array}$ & Non-smoker 75 (34\%) & Non-smoker $32(27 \%)$ \\
\hline & $\begin{array}{l}\text { Current smoker } 83 \\
(24 \%)\end{array}$ & $\begin{array}{l}\text { Current smoker } 54 \\
(24 \%)\end{array}$ & $\begin{array}{l}\text { Current smoker } 29 \\
(25 \%)\end{array}$ \\
\hline & Ex-smoker 48 (14\%) & Ex-smoker 32 (14\%) & Ex-smoker 16 (14\%) \\
\hline & Missing 101 (30\%) & Missing 62 (28\%) & Missing 39 (34\%) \\
\hline
\end{tabular}

Data expressed as mean \pm standard deviation and median [interquartile range] as appropriate; *NRS = Numerical Rating Scale. 
TABLE 7.2:

Success as judged by a patient undergoing remedial surgery for persistent groin pain syndromes (univariate binary logistic regression analysis). Effect on outcome: success or not.

\begin{tabular}{|c|c|c|c|c|}
\hline Variable & $\mathbf{n}$ & Odds ratio & $\begin{array}{l}\mathbf{9 5 \%} \\
\text { Confidence } \\
\text { Interval }\end{array}$ & p-value \\
\hline Gender & 339 & & & \\
\hline Male & 215 & Reference & Reference & Reference \\
\hline Female & 124 & 0.47 & $0.30-0.75$ & $<0.01^{* * *}$ \\
\hline Age category & 339 & & & \\
\hline$\leq 30$ & 29 & 0.38 & $0.16-0.90$ & $0.03^{* *}$ \\
\hline $31-40$ & 60 & 0.61 & $0.31-1.21$ & 0.16 \\
\hline $41-50$ & 83 & 1.29 & $0.66-2.52$ & 0.45 \\
\hline $51-60$ & 85 & Reference & Reference & Reference \\
\hline $61-70$ & 49 & 1.06 & $0.49-2.26$ & 0.89 \\
\hline$>70$ & 33 & 1.07 & $0.45-2.56$ & 0.88 \\
\hline $\mathrm{BMI}^{+}$ & 230 & 1.02 & $0.95-1.10$ & 0.60 \\
\hline Index operation & 339 & & & \\
\hline Inguinal hernia repair & 217 & Reference & Reference & Reference \\
\hline Other & 122 & 0.63 & $0.40-1.00$ & $0.05^{* *}$ \\
\hline Preoperative pain intensity $^{\dagger}$ & 200 & 0.91 & $0.75-1.11$ & 0.37 \\
\hline Duration of pain ${ }^{\dagger}$ & 318 & 1.00 & $0.99-1.00$ & $0.03^{* *}$ \\
\hline Anaesthesia & 339 & & & \\
\hline General & 201 & Reference & Reference & Reference \\
\hline Spinal & 138 & 2.38 & $1.47-3.86$ & $<0.01^{* * *}$ \\
\hline Preferred anaesthesia & 128 & & & \\
\hline General & 73 & Reference & Reference & Reference \\
\hline Spinal & 42 & 1.97 & $0.86-4.51$ & 0.11 \\
\hline Local & 6 & 0.70 & $0.13-3.69$ & 0.67 \\
\hline None & 7 & 0.93 & $0.19-4.46$ & 0.93 \\
\hline \multicolumn{5}{|l|}{ Identification of nerves } \\
\hline Ilioinguinal nerve & 330 & & & \\
\hline Yes & 262 & 0.64 & $0.35-1.15$ & 0.14 \\
\hline No & 68 & Reference & Reference & Reference \\
\hline Iliohypogastric nerve & 329 & & & \\
\hline Yes & 105 & 0.71 & $0.44-1.15$ & 0.16 \\
\hline No & 224 & Reference & Reference & Reference \\
\hline Genitofemoral nerve & 336 & & & \\
\hline Yes & 104 & 1.87 & $1.11-3.13$ & $0.02^{* *}$ \\
\hline No & 232 & Reference & Reference & Reference \\
\hline Meshoma & 336 & & & \\
\hline Yes & 43 & 3.00 & $1.29-6.98$ & $0.01 * *$ \\
\hline No & 293 & Reference & Reference & Reference \\
\hline Neuroma & 313 & & & \\
\hline Yes & 24 & 2.81 & $0.94-8.45$ & $0.07^{*}$ \\
\hline No & 289 & Reference & Reference & Reference \\
\hline Preoperative opioid use & 269 & & & \\
\hline Yes & 69 & 0.40 & $0.23-0.71$ & $<0.01^{* * *}$ \\
\hline No & 200 & Reference & Reference & Reference \\
\hline
\end{tabular}

${ }^{*} p \leq 0.1 ;{ }^{* *} p \leq 0.05 ;{ }^{* * *} p \leq 0.01 ;{ }^{+}$Continuous variable. 
TABLE 7.3:

Factors determining success after remedial surgery for groin pain syndromes (multivariate logistic regression analysis).

\begin{tabular}{|c|c|c|c|c|}
\hline Variable & $\mathbf{n}$ & Odds ratio & $\begin{array}{l}95 \% \\
\text { Confidence } \\
\text { Interval }\end{array}$ & p-value \\
\hline \multicolumn{5}{|l|}{ Gender } \\
\hline Male & 186 & Reference & Reference & Reference \\
\hline Female & 70 & 0.54 & $0.30-1.00$ & $0.01 *$ \\
\hline \multicolumn{5}{|l|}{ Age category } \\
\hline$\leq 30$ & 14 & 0.46 & $0.14-1.59$ & 0.22 \\
\hline $31-40$ & 43 & 0.50 & $0.21-1.18$ & 0.11 \\
\hline $41-50$ & 61 & 1.35 & $0.60-3.05$ & 0.47 \\
\hline $51-60$ & 70 & Reference & Reference & Reference \\
\hline $61-70$ & 40 & 1.08 & $0.43-2.70$ & 0.87 \\
\hline$>70$ & 28 & 0.83 & $0.29-2.32$ & 0.72 \\
\hline Duration of pain ${ }^{+}$ & 256 & 1.00 & $1.00-1.00$ & 0.27 \\
\hline \multicolumn{5}{|l|}{ Anaesthesia } \\
\hline General & 139 & Reference & Reference & Reference \\
\hline Spinal & 117 & 1.89 & $1.06-3.38$ & $0.03^{*}$ \\
\hline \multicolumn{5}{|l|}{ Identification n.GF } \\
\hline Yes & 82 & 1.13 & $0.59-2.19$ & 0.71 \\
\hline No & 174 & Reference & Reference & Reference \\
\hline \multicolumn{5}{|l|}{ Meshoma } \\
\hline Yes & 40 & 2.85 & $1.11-7.31$ & $0.03^{*}$ \\
\hline No & 216 & Reference & Reference & Reference \\
\hline \multicolumn{5}{|l|}{ Opioids } \\
\hline Yes & 66 & 0.44 & $0.24-0.81$ & $<0.01^{* *}$ \\
\hline No & 190 & Reference & Reference & Reference \\
\hline
\end{tabular}

${ }^{*} p \leq 0.05 ;{ }^{* *} p \leq 0.01 ;{ }^{+}$Continuous variable; ${ }^{*} n . G F=$ genitofemoral nerve. 
The number of patients qualifying for the multivariable analysis were 256 (table 7.3). After correction for confounders, spinal anaesthesia and removal of a meshoma showed a significant positive association with success (OR 1.9 and 2.9, respectively). Conversely, preoperative use of opioids and female gender reduced the chance on an advantageous outcome (OR 0.4 and 0.5 respectively). The model had an overall $68 \%$ positive predictive value. In other words, patients not using opioids undergoing meshoma removal under spinal anaesthesia have a $68 \%$ chance on a successful outcome. The sensitivity analysis, the OR for spinal anaesthesia compared to general anaesthesia corrected for all confounders selected based on expert opinion, was 1.5 and was not statistically significant (95\%CI $0.7-3.2)$.

\section{Secondary outcome}

Adverse effects of the remedial surgery included haematoma requiring reoperation (1\%), wound infection necessitating antibiotics (2\%) and testicular atrophy (1\%).

The effects of spinal anaesthesia on outcome following a neurectomy was significant (OR 2.3; table 7.4). In the other remedial operations, spinal anaesthesia also demonstrated a positive effect on outcome albeit not statistically significant. The correlation between anaesthetic technique as preferred by the patient and indeed undergone was $0.68(\mathrm{p}<0.01)$.

TABLE 7.4:

Associations between type of remedial surgery and success, represented by the odds ratio.

\begin{tabular}{lllll}
\hline Type of remedial surgery & $\mathbf{n}$ analyzed & OR & $\begin{array}{l}\mathbf{9 5 \%} \\
\text { Confidence } \\
\text { Interval }\end{array}$ & $\begin{array}{l}\text { Significance } \\
\text { level (p-value) }\end{array}$ \\
\hline Total & 339 & 2.38 & $1.47-3.86$ & $<0.01^{* *}$ \\
Neurectomy & 235 & 2.27 & $1.27-4.07$ & $<0.01^{* *}$ \\
Neurectomy + mesh removal & 88 & 1.22 & $0.42-3.52$ & 0.72 \\
Mesh removal & 15 & 4.00 & $0.27-58.56$ & 0.31 \\
Funiculolysis & 1 & & & \\
\hline Primary operation & $\mathbf{n}$ analyzed & $\mathbf{O R}$ & $\mathbf{9 5 \% C l}$ & $\begin{array}{l}\text { Significance } \\
\text { level (p-value) }\end{array}$ \\
\hline Lichtenstein repair & 171 & 2.20 & $1.13-4.32$ & $0.02^{*}$ \\
Pfannenstiel incision & 64 & 2.76 & $0.78-9.78$ & 0.12 \\
\hline
\end{tabular}

${ }^{*} p \leq 0.05 ;{ }^{* *} p \leq 0.01$ 


\section{DISCUSSION}

The present paper aimed to determine whether spinal anaesthesia is preferable over general anaesthesia in 339 patients with postoperative inguinodynia. Such an analysis has not previously been performed in this patient population or pain domain. The results are intriguing as this form of remedial surgery is suggested to have two times higher odds (OR 1.9) of a successful outcome if performed under spinal anaesthesia. This effect is the most evident in a surgical procedure termed neurectomy (removal of nerve, OR 2.3). Removal of wrinkled mesh (meshoma, OR 2.9) may also increase the probability of pain-free outcome. Conversely, opioid use and female gender reduce the odds of pain relief (OR 0.4 and 0.5 ). Adverse effects and complication rates of remedial surgery are acceptable.

The underlying potential 'protective' mechanism of a regional anaesthetic technique in these patients is not clear. Possible long-lasting effects of spinal anaesthetic agents in relation to postoperative pain cannot be explained by their pharmacologic properties only. As an extended pharmacological action seems unlikely, alternative mechanisms must prevail. During surgery, tissue damage may generate several powerful noxious impulses at the operation $\operatorname{site}^{10}$. These nociceptive signals have the ability to modify the nervous system ('neuronal plasticity'). Peripheral nerve hyperexcitability may subsequently be caused by a lower threshold of nociceptor afferent peripheral terminals, a phenomenon termed peripheral sensitization ${ }^{14}$. An extensive quantity and ongoing nociceptive input resulting from surgery may have resulted in hyperexcitability of the spinal cord and morphological changes more proximal in the central nervous system ${ }^{15,16}$. Since the release of inflammatory and proteolytic mediators into the wound may continue postoperatively, the hyperexcitable state of these structures is maintained ${ }^{10}$. Spinal anaesthesia during remedial surgery induces peripheral afferent nerve blocks and may consequently reduce nociceptive transmission from the operation site toward the central nervous system $^{10,17}$. An ongoing hyperexcitable state of the nervous system responsible for chronic postoperative pain may thus be interrupted ${ }^{10}$. Findings of the present study support this hypothesis. However, the suggestion that just nociceptive stimuli lead to either peripheral or central sensitization is not confirmed as neurectomies were also found to benefit from spinal anaesthesia. 
The present retrospective study shows that effects of spinal anaesthesia in remedial groin pain surgery are possibly less manifest in patients undergoing a meshectomy. This surgery is performed in a subgroup of groin hernia patients presenting with pain with mainly nociceptive or inflammatory pain characteristics ${ }^{18}$. In theory, the inflammatory responses evoked by the mesh itself may be more intense in this subgroup compared to patients suffering from a pure neuropathic pain. Consequently, the number of nociceptive stimuli may be more powerful and may last longer. Moreover, removal of a meshoma appears very successful since, hypothetically, removal of the cause of nociceptive stimuli may immediately result in relief of groin pain. Both factors may potentially explain a less beneficial effect of spinal anaesthesia itself on a successful outcome.

A different type of central sensitization associated with mesh-related pain versus neuropathic pain may possibly explain the differential effect of spinal anaesthesia. Prior to remedial surgery, inguinal nerves of 'neurectomy patients' are injured due to an iatrogenic or other trauma. Nerve lesions may lead to molecular changes in nociceptive neurons (C-fibres) and non-nociceptive neurons (A $\delta$ - or $A \beta$-fibres) by releasing growth factors ${ }^{14}$. These nerve growth factors were found to influence neighbouring uninjured nerves, which react by an upgraded expression of certain receptors such as vanilloid receptors (trpV1 receptors, responding to noxious heat), adrenoceptors (adrenergic receptors, responding to catecholamines) and sodium channels ${ }^{14,19-21}$. By an upregulated expression of these receptors, hyperexcitability of initially normal surrounding nerves may ensue. Furthermore, a hyperexcitable state may result in an ongoing sensitization of the spinal cord dorsal horn neurons by releasing glutamate acting on postsynaptic $\mathrm{N}$-methyl-D-aspartate (NMDA) receptors ${ }^{14}$. Both mechanisms may contribute to ectopic activity of the concerning nerves and consequent spontaneous pain ${ }^{14,22}$. Since neighbouring, initially uninjured nerves have intact connections to the skin, pain is often experienced at superficial groin areas as also reflected by a painful skin pinching test.

The presently suggested effects of type of anaesthesia may only account for specific aetiologies in persistent groin pain. Peripheral and central nervous system involvement of various groin pain syndromes may vary widely ${ }^{23}$. Physical examination might aid in distinguishing between type of pain syndrome. Unfortunately, identifying the specific injured nerve is difficult as the underlying pathophysiologic changes do not respect the anatomy of nerve, root or segmental regions as proposed in anatomy books ${ }^{14}$. Furthermore, secondary hyperalgesia (a spread of hypersensitivity to initially unaffected nerves) as 
detected during a physical examination can also trick a pain specialist ${ }^{15}$. These limitations may explain suboptimal success rates (approximately 70\%) following remedial surgery ${ }^{7,18}$. These findings are useful for preoperative counselling and the informed consent process.

Choice of anaesthesia depended on preferences of patient as well as attending anaesthesiologist and surgeon. To determine the influence of the patients' choice (and the underlying psychological factors) on outcome, the preferred anaesthetic technique was also analyzed. However, no significant correlation was found. Still, the influence of psychology on pain is known to have a large impact on outcome $e^{13,24}$.

\section{Limitations}

The present study potentially has limitations including selection bias, in particular referral bias. Since the study was performed in a tertiary referral centre, most patients had an unusually complicated history. As a consequence, less complicated patients may have been treated successfully by injection therapy or a neurectomy in other hospitals. The follow-up period was relatively short and patients may have developed pain after the follow-up period. However, the majority of patients is referred to our centre once again if pain has recurred and these results are included in the present study. To determine the association between spinal anaesthesia and a successful outcome, a variety of statistical methods may be considered. An alternative approach is to calculate ORs as assessed by multivariable analysis that corrects for all confounding factors as determined by experts. We performed this method as a means to test whether results are robust. Following calculations, the 'fully adjusted OR' for spinal anaesthesia was not significant. This outcome suggests that the present findings should be interpreted with caution. It is impossible to determine if the multivariable analysis or the fully adjusted is the best strategy to calculate the association.

Esketamine was given intraoperatively to the majority of patients in the general anaesthesia-group. Previous research has suggested this to be a confounding factor ${ }^{25}$. However, if esketamine did influence outcomes, an underestimation of the actual effect size of spinal anaesthesia on pain reduction would result. Therefore, the conclusion is likely not influenced by this potential confounder ${ }^{25}$.

The number of patients in which a meshoma or neuroma were identified, was relatively limited. As a consequence, the confidence intervals are wide and this may have considerably influenced the outcomes of the logistic regression analysis. Another limitation 
of the present study is associated with its retrospective character and a consequent lack of control on the determinant (type of anaesthesia). Data were obtained from electronic patient file entries as documented at the postoperative control by one of three attending surgeons. However, a $66 \%$ success rate is in line with previous results ${ }^{7}$. Furthermore, the anaesthesiologist determined which technique was preferable in a patient. The present study was a hypothesis generating study and outcomes may potentially be due to the mechanisms described. Therefore, a future randomized trial that is currently underway is necessary for more robust conclusions (trial registered at www.trialregister.nl, NTR registration number: 5586$)$.

\section{CONCLUSIONS}

This retrospective cohort study is the first to determine the effect of type of anaesthesia and other patient- and surgery-related factors on treatment outcome in patients undergoing remedial surgery for various types of chronic inguinodynia. The findings may indicate that pain relief after surgery under spinal anaesthesia is better, particularly if a neurectomy is performed. However, the type of approach to select confounders had an effect on our conclusion. A randomized controlled trial is required possibly to confirm these preliminary findings. 


\section{REFERENCES}

1. Gultekin FA, Kurukahvecioglu O, Karamercan A, et al. A prospective comparison of local and spinal anesthesia for inguinal hernia repair. Hernia 2007;11(2):153-6.

2. Vizcaino-Martinez L, Gomez-Rios MA, Lopez-Calvino B. General anesthesia plus ilioinguinal nerve block versus spinal anesthesia for ambulatory inguinal herniorrhapy. Saudi J Anaesth 2014;8(4):523-8.

3. Williams BA. For outpatients, does regional anesthesia truly shorten the hospital stay, and how should we define postanesthesia care unit bypass eligibility? Anesthesiology 2004;101(1):3-6.

4. Loos MJ, Scheltinga MR, Roumen RM. Surgical management of inguinal neuralgia after a low transverse Pfannenstiel incision. Ann Surg 2008;248(5):880-5.

5. Licheri S, Pisano G, Erdas E, et al. Endometriosis of the round ligament: description of a clinical case and review of the literature. Hernia 2005;9(3):294-7.

6. Hohenfellner R. Nerve injuries in urological surgery. Georgian Med News 2007(143):7-11.

7. Loos MJ, Scheltinga MR, Roumen RM. Tailored neurectomy for treatment of postherniorrhaphy inguinal neuralgia. Surgery 2010;147(2):275-81.

8. Bay-Nielsen M, Perkins FM, Kehlet H. Pain and functional impairment 1 year after inguinal herniorrhaphy: a nationwide questionnaire study. Ann Surg 2001;233(1):1-7.

9. Ozgun H, Kurt MN, Kurt I, et al. Comparison of local, spinal, and general anaesthesia for inguinal herniorrhaphy. Eur J Surg 2002;168(8-9):455-9.

10. Tverskoy M, Cozacov C, Ayache M, et al. Postoperative pain after inguinal herniorrhaphy with different types of anesthesia. Anesth Analg 1990;70(1):29-35.

11. Zwaans WA, Verhagen T, Roumen RM, et al. Factors Determining Outcome After Surgery for Chronic Groin Pain Following a Lichtenstein Hernia Repair. World J Surg 2015;39(11):265262.

12. von Elm E, Altman DG, Egger M, et al. Strengthening the Reporting of Observational Studies in Epidemiology (STROBE) statement: guidelines for reporting observational studies. BMJ 2007;335(7624):806-8.

13. Macrae WA. Chronic pain after surgery. Br J Anaestsh 2001;87(1):88-98.

14. Baron R. Mechanisms of disease: neuropathic pain--a clinical perspective. Nat Clin Pract Neurol 2006;2(2):95-106.

15. Woolf CJ, Chong MS. Preemptive analgesia--treating postoperative pain by preventing the establishment of central sensitization. Anesth Analg 1993;77(2):362-79. 
16. Sugimoto T, Takemura M, Sakai A, et al. Rapid transneuronal destruction following peripheral nerve transection in the medullary dorsal horn is enhanced by strychnine, picrotoxin and bicuculline. Pain 1987;30(3):385-93.

17. Kitahata LM. Pain pathways and transmission. Yale J Biol Med 1993;66(5):437-42.

18. Zwaans WA, Perquin CW, Loos MJ, et al. Mesh removal and selective neurectomy for persistent groin pain following Lichtenstein repair. World J Surg 2017;41(3):701-12.

19. Hudson LJ, Bevan S, Wotherspoon G, et al. VR1 protein expression increases in undamaged DRG neurons after partial nerve injury. Eur J Neurosci 2001;13(11):2105-14.

20. Wu G, Ringkamp M, Hartke TV, et al. Early onset of spontaneous activity in uninjured C-fiber nociceptors after injury to neighboring nerve fibers. J Neurosci 2001;21(8):RC140.

21. Finnerup NB, Jensen TS. Mechanisms of disease: mechanism-based classification of neuropathic pain-a critical analysis. Nat Clin Pract Neurol 2006;2(2):107-15.

22. Vecsei L, Majlath Z, Balog A, et al. Drug targets of migraine and neuropathy: treatment of hyperexcitability. CNS Neurol Disord Drug Targets 2015;14(5):664-76.

23. Palomba R, Bonaccia $\mathrm{P}$, Graffi $\mathrm{M}$, et al. The novel therapeuthic targets in the treatment of chronic pain. Transl Med UniSa 2012;3:57-61.

24. Perkins FM, Kehlet H. Chronic pain as an outcome of surgery. A review of predictive factors. Anesthesiology 2000;93(4):1123-33.

25. Chaparro LE, Smith SA, Moore RA, et al. Pharmacotherapy for the prevention of chronic pain after surgery in adults. Cochrane Database Syst Rev 2013;7:CD008307. 



\section{CASEII}

Symptomatic attachment of appendix vermiformis following a plug inguinal hernia repair

W.A.R. Zwaans

M.R.M. Scheltinga

R.M.H. Roumen

Under review 


\section{CASE REPORT}

In 2010, a 62-year-old man with a history of a right upper lobectomy for lung cancer visited our outpatient clinic with severe groin pain. Some ten years earlier, a right inguinal hernia was repaired by the Rutkow-Robbins mesh plug approach. During this technique, a polypropylene plug is positioned in the internal inguinal ring. In 2008, he started to experience a progressively painful burning sensation in the right groin area and the inner part of his upper leg. Dysejaculation, painful erections and transient electric shocks accompanied this pain syndrome that was diagnosed as chronic inguinal postoperative pain (CPIP). Imaging including ultrasound and CT-scan did not demonstrate any particular causes for his pain complex.

At physical examination, altered right-sided scrotal skin sensibility and a locally painful pinching were demonstrated whereas a palpable lump in his right groin were palpated. During palpation, the pain was irradiating towards his upper leg. It was concluded that his CPIP consisted of a neuropathic pain syndrome due to entrapment of the ilioinguinal nerve and possibly the genital branch of the genitofemoral nerve. At that time, it was unclear whether a winkled mesh contributed to the pain. He consented to an operative exploration of the right groin. Intraoperatively, the ilioinguinal and the genital branch of the genitofemoral nerve were encased by the plug. A double neurectomy was executed in combination with a partial meshectomy and funiculolysis. Deeper portions of the mesh were left in situ to prevent a recurrent hernia. At the postoperative outpatient control, the patient was pain free.

Five years later, the patient was again presented to our department of SolviMáx with painful urge of miction, abdominal discomfort with nausea and a progressive sensation of tightness in the right groin area that had developed over the last two years. He was convinced that he suffered from dyspepsia or gastritis although medication were to no avail. A previous gastroscopy had revealed no abnormalities. Groin skin neuropathic characteristics were not present whereas pinching of the skin was also not painful. However, a small but painful lump was palpable deep within the groin area. A Valsalva manoeuvre was negative suggesting absence of a recurrent hernia. A nociceptive mesh-related pain was diagnosed and a second exploration was planned. The palpable lump appeared the Rutkow-Robbins plug that was now positioned deeply and mainly preperitoneally into the inguinal canal. Following plug dissection, the appendix vermiformis was found attached 
to the meshoma (figure II.1). The plug was also firmly attached to bladder, spermatic cord and epigastric vessels. A meshectomy and an appendectomy were performed whereas the latter structures could be dissected without structural damage. Histopathological examination demonstrated a giant cell reaction in the presence of the foreign body and ingrowth into the submucosa of the appendix.

Postoperatively, the patient was successfully treated with antibiotics for a low grade wound infection. At the outpatient evaluation, the groin pain syndrome and foreign body feeling disappeared and the patient was very satisfied. Surprisingly, he spontaneously mentioned himself that his painful urge and nausea with abdominal discomfort were completely gone.
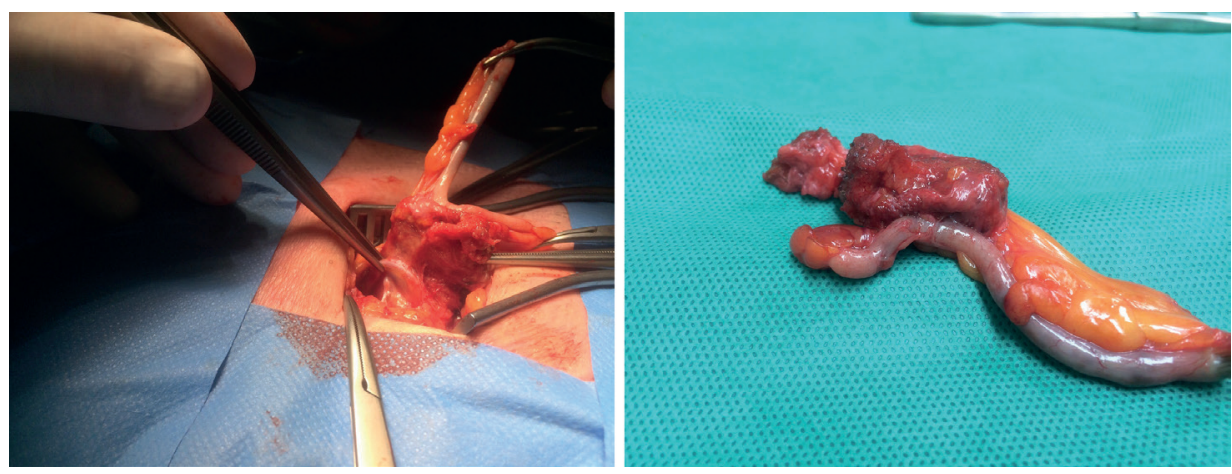

FIGURE II.1:

Intraoperative views of the appendix vermiformis firmly attached to Rutkow/Robbins plug that was previously used for an inguinal hernia repair. The left forceps is holding the bladder.

\section{DISCUSSION}

In the year of 2017, the gold standard for inguinal hernia repair is still open or laparoscopic mesh repair, depending on resources and available expertise ${ }^{1}$. Plug repairs are nowadays considered inferior since these techniques include the excessive use of foreign material, enter both posterior and anterior inguinal planes and result in additional costs ${ }^{1}$. Furthermore, plugs have a greater tendency to migrate when compared to flat meshes ${ }^{1}$. An easier migration of plugs may be due to inadequate fixation. It is 
therefore advised to fixate the plug with non-absorbable suture material. A second cause of migration of prosthetic material is related to a foreign body reaction ${ }^{2}$. These reactions may lead to erosion of the tissue surrounding the plug (or mesh, in a similar configuration called a meshoma). Grade of erosion highly depends on the biocompatibility of the material $^{2,3}$. Other factors influencing this biocompatibility include type of material, pore size and rigidity of the plug or mesh $^{3}$. Irrespective of the mechanism of erosion, invasion of plugs (or meshes) into the viscera of the gastrointestinal tract, urinary bladder or even vessels is a potentially devastating long-term complication. The present patient reported a painful urge of miction that was eventually due to his plug progressively invading the bladder. Although rare, previous cases of intestinal obstruction and/or perforation due to migration of meshes have been described ${ }^{2}$.

If postherniorrhaphy visceral complaints present a number of years after a meshbased repair, migration of the prosthetic material compromising the viscera should be considered in the differential diagnosis. In the present case, the appendix was not inflamed or macroscopically altered during surgical exploration. Although a convincing appendicopathy was macroscopically also absent, external compression of the adjacent mesh may have lead to some form of inflammatory reaction and pain $^{4}$ or a neuromodulative response $\mathrm{e}^{5}$. In the present case it was hypothesized that the plug had migrated from the inguinal canal into the peritoneal cavity and eventually adhered to the appendix. This process eventually resulted in abdominal discomfort and nausea via a reflex mechanism. This phenomenon is a type of central somatovisceral integration which can result in this specific type of referred visceral pain. These complaints may typically mimic stomach ache, although the pain is resistant to stomach-protecting medications as indicated by the patient. Such discomfort is a characteristic example of a segmentally related complaint. Removal of the meshoma-appendix trigger continuously supporting this afferent input resulted in an immediate relief of the visceral discomfort. 


\section{CONCLUSION}

Migration and erosion of meshes and plugs are rare complications of inguinal hernia repair but may lead to severe complications. Early recognition may prevent perforations of the viscera. Postherniorrhaphy pain may be due to a less recognized phenomenon termed referred visceral pain. Physicians should think of this possible aetiology of referred pain. Mesh or plug removal is the appropriate surgical treatment once prosthetic material has migrated.

\section{REFERENCES}

1. Simons MP, Smietanski M, Bonjer HJ, et al. World guidelines for groin hernia management. 2016.

2. Agrawal A, Avill R. Mesh migration following repair of inguinal hernia: a case report and review of literature. Hernia 2006;10(1):79-82.

3. Bellon JM, Garcia-Carranza A, Garcia-Honduvilla N, et al. Tissue integration and biomechanical behaviour of contaminated experimental polypropylene and expanded polytetrafluoroethylene implants. Br J Surg 2004;91(4):489-94.

4. Jennings JM, Ng P. Laparoscopic appendicectomy for suspected mesh-induced appendicitis after laparoscopic transabdominal preperitoneal polypropylene mesh inguinal herniorraphy. J Minim Access Surg 2010;6(1):19-21.

5. Ballas K, Kontoulis T, Skouras C, et al. Unusual findings in inguinal hernia surgery: report of 6 rare cases. Hippokratia 2009;13(3):169-71. 



\title{
CHAPTER 8
}

Spinal versus general anaesthesia in surgery for inguinodynia (SPINASIA trial): study protocol for a randomized controlled trial

\author{
W.A.R. Zwaans
}

L.H.P.M. le Mair

M.R.M. Scheltinga

R.M.H. Roumen

Trials 2017; 18: 23 


\section{ABSTRACT}

Background: Chronic inguinodynia (groin pain) is a common complication following open inguinal hernia repair or a Pfannenstiel incision but may also be found after other types of (groin) surgery. If conservative treatments are to no avail, tailored remedial surgery, including a neurectomy and/or a (partial) meshectomy, may be considered. Retrospective studies in patients with chronic inguinodynia suggested that spinal anaesthesia is superior compared to general anaesthesia in terms of pain relief following remedial operations. This randomized controlled trial is designed to study the effect of type of anaesthesia (spinal or general) on pain relief following remedial surgery for inguinodynia.

Methods: A total of 190 adult patients who suffer from unacceptable chronic (>3 months) inguinodynia, as subjectively judged by patients themselves, are included. Only patients scheduled to undergo a neurectomy and/or a meshectomy by an open approach are considered for inclusion and randomized to spinal or general anaesthesia. Patients are excluded if pain is attributable to abdominal causes or if any contra-indications for either type of anaesthesia are present. Primary outcome is the effect of type of anaesthesia on pain relief. Secondary outcomes include patient satisfaction, quality of life, use of analgesics and (in)direct medical costs. Patient follow-up is one year.

Discussion: The first patient was included in January, 2016. Expected trial deadline is December 2019. Potential effects are deemed related to the entire setting of type of anaesthesia. Since any setting is multifactorial, all of these factors may influence the outcome measures.

This is the first large randomized controlled trial comparing the two most frequently used anaesthetic techniques in remedial surgery for groin pain. There is a definite need for evidence-based strategies to optimize results of types of surgery. Besides pain relief, other important patient-related outcome measures are assessed to include patient's perspectives on outcome. 
Trial registration: The protocol (protocol number NL54115.015.15) is approved by the Medical Ethics Committee of Máxima Medical Centre, Veldhoven, the Netherlands. The study protocol is registered at www.trialregister.nl (NTR registration number: 5586), date of registration 15th of January, 2016. 


\section{INTRODUCTION}

\section{Background and objectives}

Chronic postoperative groin pain (inguinodynia) is defined as pain beyond three months after inguinal surgery. In the majority of patients, inguinodynia develops following open inguinal hernia repair (66\%; Zwaans WA, van Kuijk SM, le Mair LH, van Kleef M, Scheltinga MR, Roumen RM - unpublished observations). For instance, one third of patients with chronic postherniorrhaphy pain experience impairment of daily activities ${ }^{1,2}$. This pain is either neuropathic (47-70\%), nociceptive (26\%) or a combination $^{3}$. Inguinodynia may also be found after other types of groin surgery ${ }^{4-7}$. Concise history taking and an extensive physical examination provide the corner stones in diagnosing inguinodynia. A diagnostic local nerve block may confirm the diagnosis ${ }^{8,9}$. Interestingly, one of three patients benefits from these injections on the long-term ${ }^{10}$. When these minimally invasive regimens fail, surgical options may be considered. Removal of inguinal nerves, funiculolysis, and/or removal of the mesh may all be effective ${ }^{7,11-14}$. Furthermore, the post-Pfannenstiel pain syndrome, which often is neuropathic in origin, also responds well to an inguinal neurectomy ${ }^{4}$.

The SolviMáx centre of excellence is a third line referral centre for abdominal wall and groin pain. Experience with a spectrum of surgical procedures for inguinodynia is building. A retrospective study in patients with inguinodynia suggested that spinal anaesthesia is associated with a superior outcome in terms of pain relief when compared to general anaesthesia ${ }^{15}$. A more robust trial is required to confirm these preliminary findings. Objective of the present paper is to present a randomized controlled trial that is designed to investigate the effect of type of anaesthesia on long term pain relief in patients who are surgically treated for chronic inguinodynia.

\section{METHODS}

\section{Trial design}

This mono-center, non-blinded, randomized controlled trial is performed in the SolviMáx Centre of Excellence for Abdominal Wall and Groin Pain. SolviMáx is a subdivision of the surgical department of Máxima Medical Center (MMC), a teaching 
hospital situated in the southern part of The Netherlands serving a population of approximately 200,000 inhabitants. The study aims at investigating a potential difference in pain relief following remedial surgery that is performed in two different settings, spinal anaesthesia versus general anaesthesia. The present trial is not considered a drug study as other variables associated with type of anaesthesia are not under control of the investigators. The present trial follows guidelines of the declaration of Helsinki (version October 19th, 2013). The protocol (protocol number NL54115.015.15) is approved by the Medical Ethics Committee of Máxima Medical Centre, Veldhoven, the Netherlands. The study protocol (version 1) is registered at www.trialregister.nl (NTR identification number: 5586). The present paper is written according to the SPIRIT 2013 statement for reporting a clinical trial protocol ${ }^{16}$. The SPIRIT checklist is provided as an additional file.

\section{Participants}

Patient enrolment started in January, 2016. Chronic inguinodynia that is unacceptable as subjectively judged by patients themselves is a prerequisite for inclusion. Only patients scheduled to undergo remedial surgery including a neurectomy and/or a meshectomy (if patients have mesh) by an open approach are considered for inclusion (figure 8.1). Patients are excluded if pain is due to intercostal neuralgia of the abdominal wall, such as abdominal cutaneous nerve entrapment syndrome (ACNES) or due to the lateral femoral cutaneous nerve entrapment. Other contra-indications are listed in table 8.1. Cognitive impaired individuals, patients with ASA class IV or undergoing secondary or bilateral remedial surgery are also excluded.

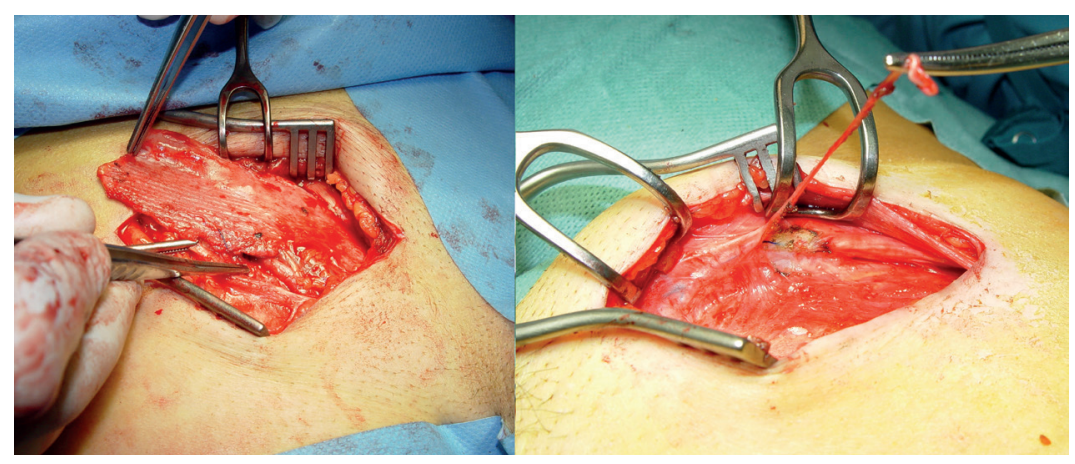

FIGURE 8.1:

Mesh removal (left) and a neurectomy (right) in patients with inguinodynia following open inguinal hernia repair. 
TABLE 8.1:

Contra-indications for spinal and general anaesthesia in the present trial

\begin{tabular}{ll}
\hline Relative contra-indications & Absolute contra-indications \\
\hline Spinal anaesthesia & \\
\hline Deformities of the spine* & Refusal by patient \\
Severe back pain or headache & Inadequate coagulation \\
& Pre-existent neurological deficiency \\
& Infection near puncture site \\
& Allergy for local anaesthetic \\
& Inability to communication properly \\
\hline Eeneral anaesthesia & \\
\hline Expected difficulty with airway & Refusal by patient \\
& Allergy for (components of) medications
\end{tabular}

*E.g. arthritis, osteoporosis, metastasis, spinal disc herniation, scoliosis

\section{Interventions}

The decision to perform a neurectomy and/or mesh removal depends on the surgeon's subjective interpretation of patient history, physical examination and intraoperative findings ${ }^{17}$. All remedial surgeries are performed using an open approach as previously published ${ }^{15}$. Patients are randomized to either spinal or general anaesthesia. Hyperbaric articaine 5\% is used for spinal anaesthesia, considering the relatively short duration of action. Articaine is a amide-type local anaesthetic agent that is preferentially used for short ( $\leq 1$ hour) surgical procedures in MMC. General anaesthesia is given to the patients following standardized procedures.

The administration of other medications during the peri-operative phase (e.g. ephedrine, atropine and sedatives) is left to the discretion of the attending anaesthesiologist. Consequently, the setting of the two types of anaesthesia rather than the actions of the anaesthetic drugs are investigated. It is allowed for patients, randomized to spinal anaesthesia group, to receive midazolam during the procedure. Midazolam has no effect on perception of pain and so, no drug-related sequels on the primary outcome measure are expected. If requested, midazolam will be administered in a dose of $1 \mathrm{mg}$ ( $>65$ years) or $2 \mathrm{mg}$ (ASA class $<\mathrm{III})$. 
If a patient receives spinal anaesthesia but intrathecal articaine has insufficient effect, the patient will undergo surgery under general anaesthesia. Subsequently, this will be considered as protocol violation and the particular patient will be excluded from further analysis.

STUDY PERIOD

\begin{tabular}{|c|c|c|c|c|c|c|c|}
\hline \multirow{2}{*}{ Enrolment } & Allocation & \multicolumn{4}{|c|}{ Postoperative follow-up } & Close-out \\
\cline { 2 - 7 } & Preoperative & 0 & $1 \mathrm{wk}$ & $6 \mathrm{wk}$ & $3 \mathrm{mo}$ & $6 \mathrm{mo}$ & $12 \mathrm{mo}$ \\
\cline { 2 - 7 }
\end{tabular}

ENROLMENT:

\begin{tabular}{r|c|c|c|c|c|c|c|}
\hline Eligibility screen & $\mathrm{X}$ & & & & & & \\
\cline { 2 - 8 } Informed consent & $\mathrm{X}$ & & & & & & \\
\cline { 2 - 8 } Allocation & & $\mathrm{X}$ & & & & & \\
\cline { 2 - 8 } & &
\end{tabular}

INTERVENTIONS:

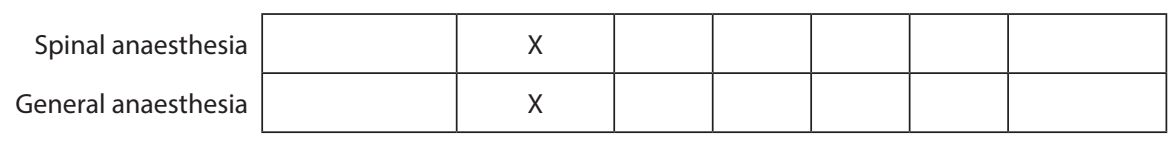

ASSESSMENTS:

Type of remedial surgery

\begin{tabular}{|c|c|c|c|c|c|c|c|}
\hline & & & & & & & \\
\hline Operation time & & $x$ & & & & & \\
\hline Pain scores & $x$ & & $x$ & $x$ & $x$ & $x$ & $x$ \\
\hline Quality of life & $x$ & & $x$ & $x$ & $x$ & $x$ & $x$ \\
\hline Patient satisfaction & & & $x$ & $x$ & $x$ & $x$ & $x$ \\
\hline Medical costs & $x$ & & & $x$ & $x$ & $x$ & $x$ \\
\hline Complications & & & $x$ & $x$ & $x$ & $x$ & $x$ \\
\hline
\end{tabular}

${ }^{*} P C S=$ Pain Catastrophizing Scale; ${ }^{* * H A D S}=$ Hospital Anxiety and Depression Scale

FIGURE 8.2:

Content for the schedule of enrolment, interventions, and assessments, according to the SPIRIT statements ${ }^{16}$. 


\section{Outcomes}

Primary outcome is the effect of type of anaesthesia on pain relief after surgery. Patients are asked to score their pain using the Numerical Rating Scale (NRS, 0, absent pain -10 , worst pain imaginable). The first pain score is obtained in the preoperative phase $\left(t_{0}\right)$, figure 8.2. Follow-up pain levels are determined at five time points to determine short-term ( 1 week, 6 weeks and 3 months postoperatively, $\mathrm{t}_{1}-\mathrm{t}_{3}$ ) and long-term results ( 6 months and 12 months postoperatively, $\mathrm{t}_{4}-\mathrm{t}_{5}$ ).

Secondary outcome measures are the effect of type of anaesthesia on quality of life and patient satisfaction. Quality of life is measured using the Short Form Health Survey-12 questionnaire (SF-12). To avoid confusion, satisfaction is also measured by NRS-like method, using a 11-point rating scale.

Other secondary endpoints include differences in pain medication and both direct and indirect medical costs. Two different validated questionnaires, developed by the institute for Medical Technology Assessment (iMTA, Erasmus University Rotterdam, www.imta. nl) are used $^{18}$. Complications (both surgery- and anaesthesia-related) are scored as based on the complication register of the Dutch Society for Anaesthesiologists. They are classified using the validated Clavien-Dindo classification ${ }^{19}$. Differences between various types of remedial surgery and specific causes of inguinodynia (primary inguinal hernia repair, primary Pfannenstiel incision, other index surgery, 'spontaneous' inguinodynia) in relation to efficacy are also determined.

\section{Sample size}

SolviMáx was founded in 2011. An increasing number of patients with potential abdominal wall or groin pain syndromes are referred to the surgical specialists (figure 8.3). Approximately 840 unique patients were analyzed in 2014, and 1045 patients in 2015. Approximately 40-45\% of these undergo remedial surgery.

Sample size was calculated by using a web-based calculator (www.openepi.com). Results from previous retrospective studies were used as criteria for eligibility were similar (Zwaans WA, van Kuijk SM, le Mair LH, van Kleef M, Scheltinga MR, Roumen RM - unpublished observations). Additional literature regarding anaesthesia on remedial surgery is not available. Previously demonstrated success rates were $77 \%$ in the spinal anaesthesia group and 58\% in the general anaesthesia group (Zwaans WA, van Kuijk SM, le Mair LH, van Kleef M, Scheltinga MR, Roumen RM - unpublished observations). 
Based on these data, a sample size of 190 patients is calculated to demonstrate a potential effect of either type of anaesthesia on pain relief. This volume of patients is attained after three years as based on the number of included patients in previously performed studies (figure 8.4).

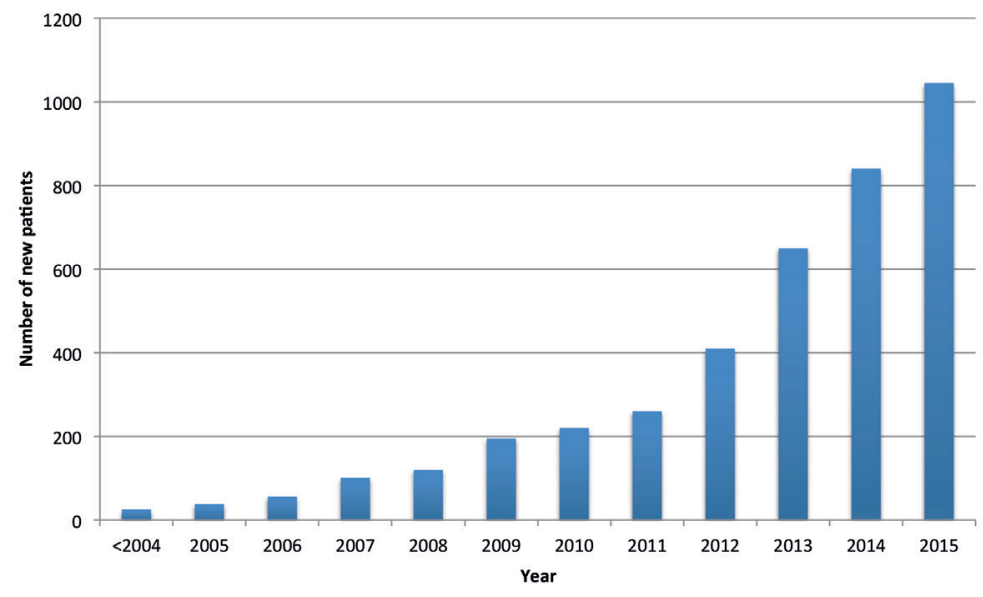

\section{FIGURE 8.3:}

Number of unique patients analyzed at SolviMáx centre of excellence for chronic abdominal wall and groin pain over the years.

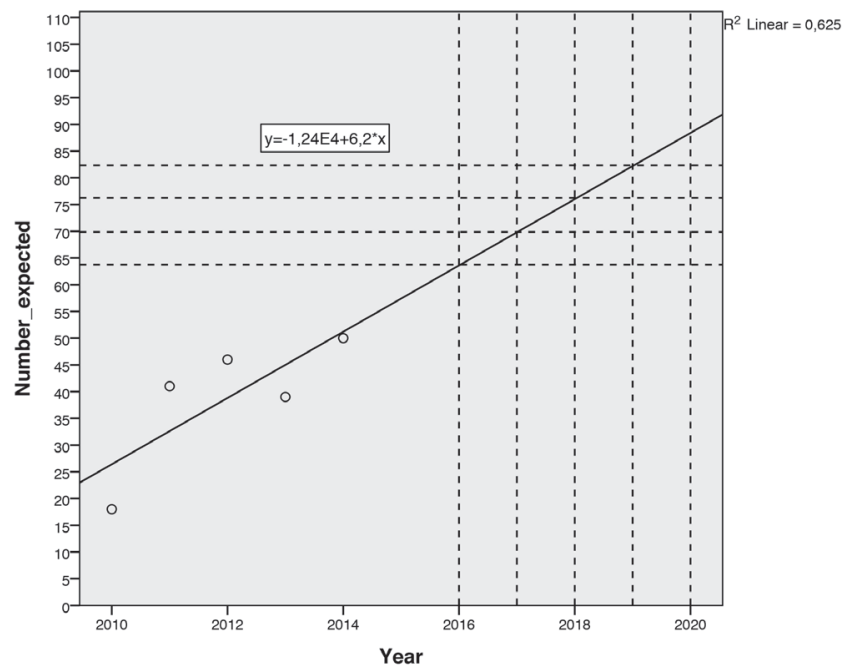

FIGURE 8.4:

Expected inclusion in the present trial, calculated by included patients in retrospective studies, previously performed ${ }^{15}$. 


\section{Randomization}

Patients will be electronically randomized by the web-based research software Research Manager (RM, Cloud 9 Health Solutions ${ }^{\odot}$ ). No blocked randomization or prestratification is performed. The decision to execute a neurectomy and/or mesh removal depends on patient history, physical examination ánd intraoperative findings ${ }^{17}$. Therefore, a prestratified randomization is not possible for type of remedial surgery. Consequently, stratification will be executed within the analysis.

\section{Sequence generation, allocation concealment and implementation}

$\mathrm{RM}$ is used to establish an appropriate sequence generation and allocation concealment. RM generates random allocation sequence, after which patients will be enrolled by the coordinating investigator. By using RM software, foreknowledge of (upcoming) anaesthesia assignments by the investigators is secured. Consequently, bias due to improper randomization techniques is minimized.

\section{Blinding}

Blinding of anaesthesiologists, surgeon and patients is not possible. Since the study is designed to investigate the setting of type of anaesthesia, where all factors associated with anaesthesia are of interest, blinding is not conditional for a robust conclusion on efficacy of either anaesthetic technique setting.

\section{Statistical methods}

Data analysis will be performed using SPSS version 22 (SPSS Inc. ${ }^{\odot}$ ) for Windows. Digital data are easily exported from RM to SPSS. The primary outcome measure is pain relief using NRS as compared to the preoperative pain levels $\left(\mathrm{t}_{0}\right)$. Pain reduction is calculated by using the following formula: PAIN RELIEF $=\left(1-\left(\mu N R S t_{x} / \mu N R S t_{0}\right)\right)^{\star} 100 \%$. Data of the general and spinal anaesthesia group will be compared using the student t-test or Wilcoxon signed ranks test, as appropriate.

Secondary outcome measures will be compared between groups at the various postoperative follow-up points. In addition, outcomes at each evaluation time $\left(t_{1}-t_{5}\right)$ will be compared to preoperative data within groups. The student t-test or Wilcoxon signed rank test will be used as appropriate. P-values $\leq 0.05$ are considered statistically significant. Intention-to-treat analysis will be applied on the primary outcome measure. 


\section{Recruitment}

Patients will be recruited at SolviMáx once the shared decision is taken to perform remedial surgery. During the subsequent preoperative screening, eligibility of patients for both general and spinal anaesthesia is assessed. Within fourteen days of consideration, informed consent is obtained. If the patient consents, the coordinating investigator will randomize the patient as previously described. Patients are allowed to retreat at any time point during the study.

\section{DISCUSSION}

General anaesthesia is still applied in the majority (60-70\%) of hernia operations ${ }^{20}$. Spinal anaesthesia, on the other hand, is only performed in 10-20\% patients undergoing inguinal hernia surger ${ }^{20}$, although beneficial effects regarding postoperative pain were previously demonstrated in primary hernia repair ${ }^{21,22}$. In addition, studies on primary inguinal herniorrhaphy showed that spinal anaesthesia results in shorter hospital stay, less postoperative analgesic requirements, prolonged time to first analgesic requirement, equal operation room time and equal time-to-home readiness ${ }^{22-24}$. Only recently, a potential beneficial effect of spinal anaesthesia on surgery for groin pain was demonstrated ${ }^{15}$. This is the first randomized trial comparing two routine anaesthetic modalities for remedial surgery. Results of the present trial may aid in optimizing care in these patient populations.

Considering groin pain relief following remedial surgery for groin pain, spinal anaesthesia is hypothesized superior to general anaesthesia. The assumed beneficial effect is possibly associated with the spinal block itself diminishing transmission of nociceptive signals from the operation site toward the nervous system ${ }^{21,25}$. However, as potential confounding factors are not excluded, a possible beneficial effect cannot be attributed to just one particular factor. Any potential effect should be considered as the end result of the total setting of type of anaesthesia and associated factors. These confounding factors include the intrathecally administration of anaesthetics, articaine or other drugs, patient expectations, surgical stress, patient's state (sedated or awake) during surgery, communication with patients and all sorts of other individual psychological factors. The present study is designed to generate clinically relevant conclusions that can be directly implemented in clinical practice. 
In 2004, Burney and co-authors performed a randomized trial on the effects of anaesthesia in primary herniorrhaphy ${ }^{26}$. A disappointingly low recruitment rate was observed. The authors stated that patients had conceptualized concerns regarding the anaesthetic techniques, and consequently did not accept a random assignment ${ }^{26}$. It is theoretically possible that patients who are more anxious about the upcoming surgical procedure prefer general over spinal anaesthesia, as they do not wish to experience any 'noise' from within the operation room. Studies have demonstrated that anxiety is a risk factor for postoperative pain ${ }^{27-29}$. When these patients are prone to more intense postoperative pain and thus prefer general anaesthesia, these issues may have influenced previous outcomes. In contrast, another study showed that preferred type of anaesthesia did not contribute to success after remedial surgery (Zwaans WA, van Kuijk SM, le Mair LH, van Kleef M, Scheltinga MR, Roumen RM - unpublished observations). In SolviMáx, a set of intake questionnaires is standard obtained from all preoperative patients including a pain catastrophizing scale (PCS) and a hospital anxiety and depression scale $(\mathrm{HADS})^{30-32}$. By analyzing these scores in relation to outcome, it is possible to analyze the influence of mental status in a later phase.

The majority of studies on chronic postoperative pain lack sufficient data to draw any robust conclusions. Previously, authors have claimed that extensive preoperative data, detailed characteristics of surgical procedures and measures on acute and chronic postoperative pain (up to one year) are essential for a proper evaluation of effects on pain $^{29}$. The present trial design has included all of these factors and therefore, the results can be considered of sufficient scientific evidence. Furthermore, cost effectiveness of both anaesthetic techniques is assessed, which is critical for clinical practice.

A potential limitation of the present study is its non-blinded design. Blinding of patients is, to our opinion, unethical and hardly practicable. Moreover, the main outcomes of the present trial are subjective. Consequently, these outcomes may be contaminated by recall bias if patients enrolled in the trial are not blinded to their treatment allocation ${ }^{33}$.

This is the first large randomized controlled trial comparing two routinely used anaesthetic techniques in remedial surgery for groin pain. There is a definite need for evidence-based strategies to optimize results of these types of surgery. Besides pain relief, other patient-related outcome measures are assessed to include patient's perspectives on outcome. The first study results are expected in 2019 and will be communicated via publication. 


\section{REFERENCES}

1. Nienhuijs S, Staal E, Strobbe L, Rosman C, Groenewoud H, Bleichrodt R. Chronic pain after mesh repair of inguinal hernia: a systematic review. Am J Surg. 2007;194(3):394-400.

2. Bay-Nielsen M, Perkins FM, Kehlet $H$. Pain and functional impairment 1 year after inguinal herniorrhaphy: a nationwide questionnaire study. Ann Surg. 2001;233(1):1-7.

3. Loos MJ, Roumen RM, Scheltinga MR. Classifying post-herniorrhaphy pain syndromes following elective inguinal hernia repair. World J Surg. 2007;31(9):1760-5. discussion 1766-7.

4. Loos MJ, Scheltinga MR, Roumen RM. Surgical management of inguinal neuralgia after a low transverse Pfannenstiel incision. Ann Surg. 2008;248(5):880-5.

5. Licheri S, Pisano G, Erdas E, Ledda S, Casu B, Cherchi MV, Pomata M, Daniele GM. Endometriosis of the round ligament: description of a clinical case and review of the literature. Hernia. 2005;9(3):294-7.

6. Hohenfellner R. Nerve injuries in urological surgery. Georgian Med News. 2007;143:7-11.

7. Aasvang EK, Kehlet $\mathrm{H}$. The effect of mesh removal and selective neurectomy on persistent postherniotomy pain. Ann Surg. 2009;249(2):327-34.

8. Lichtenstein IL, Shulman AG, Amid PK, Montllor MM. Cause and prevention of postherniorrhaphy neuralgia: a proposed protocol for treatment. Am J Surg. 1988;155(6):78690.

9. Loos MJ, Verhagen T, Scheltinga MR, Roumen RM. A randomised controlled trial of injection therapy versus neurectomy for post-herniorrhaphy inguinal neuralgia: rationale and study design. Hernia. 2010;14(6):593-7.

10. Thomassen I, van Suijlekom JA, van de Gaag A, Ponten JE, Nienhuijs SW. Ultrasound-guided ilioinguinal/iliohypogastric nerve blocks for chronic pain after inguinal hernia repair. Hernia. 2013;17(3):329-32.

11. Loos MJ, Scheltinga MR, Roumen RM. Tailored neurectomy for treatment of postherniorrhaphy inguinal neuralgia. Surgery. 2010;147(2):275-81.

12. Amid PK. A 1-stage surgical treatment for postherniorrhaphy neuropathic pain: triple neurectomy and proximal end implantation without mobilization of the cord. Arch Surg. 2002;137(1):100-4.

13. Chen DC, Hiatt JR, Amid PK. Operative management of refractory neuropathic inguinodynia by a laparoscopic retroperitoneal approach. JAMA Surg. 2013; 148(10):962-7. 
14. Bischoff JM, Enghuus C, Werner MU, Kehlet H. Long-term follow-up after mesh removal and selective neurectomy for persistent inguinal postherniorrhaphy pain. Hernia. 2013;17(3):33945.

15. Zwaans WA, Verhagen T, Roumen RM, Scheltinga MR. Factors determining outcome after surgery for chronic groin pain following a Lichtenstein hernia repair. World J Surg. 2015;39(11):2652-62.

16. Chan AW, Tetzlaff JM, Altman DG, Laupacis A, Gotzsche PC, Krleza-Jeric K, Hrobjartsson A, Mann H, Dickersin K, Berlin JA, Dore CJ, Parulekar WR, Summerskill WS, Groves T, Schulz KF, Sox HC, Rockhold FW, Rennie D, Moher D. SPIRIT 2013 Statement: defining standard protocol items for clinical trials. Ann Intern Med. 2013;158(3):200-7.

17. Zwaans WA, Perquin CW, Loos MJ, Roumen RM, Scheltinga MR. Mesh removal and selective neurectomy for persistent groin pain following Lichtenstein repair. World J Surg. 2016. doi: 10.1007/s00268-016-3780-y.

18. Hakkaart-van Roijen L, Tan SS, Bouwmans CAM. Handleiding voor kostenonderzoek. Methoden en referentieprijzen voor economische evaluaties in de gezondheidszorg. Erasmus University Rotterdam commissioned by College voor Zorgverzekeringen; 2011. Updated 2010.

19. Clavien PA, Sanabria JR, Strasberg SM. Proposed classification of complications of surgery with examples of utility in cholecystectomy. Surgery. 1992;111(5):518-26.

20. Kehlet H, White PF. Optimizing anesthesia for inguinal herniorrhaphy: general, regional, or local anesthesia? Anesth Analg. 2001;93(6):1367-9.

21. Tverskoy M, Cozacov C, Ayache M, Bradley Jr EL, Kissin I. Postoperative pain after inguinal herniorrhaphy with different types of anesthesia. Anesth Analg. 1990;70(1):29-35.

22. Ozgun H, Kurt MN, Kurt I, Cevikel MH. Comparison of local, spinal, and general anaesthesia for inguinal herniorrhaphy. Eur J Surg. 2002;168(8-9):455-9.

23. Toivonen J, Permi J, Rosenberg PH. Analgesia and discharge following preincisional ilioinguinal and iliohypogastric nerve block combined with general or spinal anaesthesia for inguinal herniorrhaphy. Acta Anaesthesiol Scand. 2004;48(4):480-5.

24. Song D, Greilich NB, White PF, Watcha MF, Tongier WK. Recovery profiles and costs of anesthesia for outpatient unilateral inguinal herniorrhaphy. Anesth Analg. 2000;91(4):876-81.

25. Kitahata LM. Pain pathways and transmission. Yale J Biol Med. 1993;66(5):437-42. 
26. Burney RE, Prabhu MA, Greenfield ML, Shanks A, O'Reilly M. Comparison of spinal vs general anesthesia via laryngeal mask airway in inguinal hernia repair. Arch Surg. 2004;139(2):183-7.

27. Aasvang E, Kehlet H. Chronic postoperative pain: the case of inguinal herniorrhaphy. Br J Anaesth. 2005;95(1):69-76.

28. Macrae WA. Chronic pain after surgery. Br J Anaesth. 2001;87(1):88-98.

29. Perkins FM, Kehlet H. Chronic pain as an outcome of surgery. A review of predictive factors. Anesthesiology, 2000. 93(4): p. 1123-33.

30. Sullivan MJ, Bishop S, Pivik J. The pain catastrophizing scale: development and validation. Psychol Assess, 1995. 7: p. 432-524.

31. White D, Leach C, Sims R, Atkinson M, Cottrell D. Validation of the Hospital Anxiety and Depression Scale for use with adolescents. Br J Psychiatry, 1999. 175: p. 452-4.

32. Spinhoven P, Ormel J, Sloekers PP, Kempen GI, Speckens AE, van Hemert AM. A validation study of the Hospital Anxiety and Depression Scale (HADS) in different groups of Dutch subjects. Psychol Med, 1997. 27(2): p. 363-70.

33. Hassan E. Recall Bias can be a Threat to Retrospective and Prospective Research Designs. The Internet Journal of Epidemiology, 2005. 3(2). 



\title{
CHAPTER 9
}

Recall bias in pain scores evaluating abdominal wall and groin pain surgery

\author{
W.A.R. Zwaans
}

J.A. de Bruijn

J.P. Dieleman

E.W. Steyerberg

M.R.M. Scheltinga

R.M.H. Roumen

Under review 


\section{ABSTRACT}

Objective: Given the importance of self-reported pain scores in clinical research, its reliability is critical. Currently, it is unclear as to whether recall of pain scores just results in inaccurate measurements or may also lead to a significantly altered outcome of retrospective studies on pain and to what extent.

Aim of the present study was to determine whether levels of preoperative pain as recalled by a patient in the postoperative phase are possibly overestimated or underestimated compared to prospectively scored pain levels. If so, a subsequent misclassification may induce recall bias that may lead to an erroneous effect outcome.

Methods: Data of seven retrospective cohort studies on surgery for chronic abdominal wall and groin pain using three different pain scores were systematically analyzed. First, it was assessed whether retrospectively acquired preoperative pain levels, as scored by the patient in the postoperative phase, differed from prospectively obtained preoperative pain scores. Secondly, it was determined if errors associated with retrospectively obtained pain scores potentially lead to a misclassification of treatment outcome. Thirdly, a meta-analysis established whether recall misclassifications, if present, affected overall study conclusions.

Results: Pain data of 313 patients undergoing remedial surgery were evaluated. The overall prevalence of misclassification due to a recall error was $13.7 \%$. Patients not benefitting from surgery ('failures') judged their preoperative pain level as more severe than it actually was. In contrast, patients who were pain free after remedial surgery ('successes') underestimated preoperative pain scores. Recall misclassifications were significantly more present in failures than in successful patients (odds ratio 2.4 [95\%CI: 1.2-4.8]).

Conclusions: One in seven patients undergoing remedial groin surgery is misclassified on the basis of retrospectively obtained preoperative pain scores (success instead of failure, or vice versa). Misclassifications are relatively more present in failures after surgery. Therefore, the effect size of a therapy erroneously depends on its success rate. 


\section{INTRODUCTION}

Bias in epidemiological studies can lead to incorrect or inconsistent conclusions. A widely accepted bias classification recognizes three main types: selection bias, information bias and confounding bias ${ }^{1}$. Correction during data analysis is only possible for the latter type, thus identification and avoidance of selection bias and information bias in the scientific research is crucial.

Recall bias is a subtype of information bias which commonly arises in retrospective studies but may also occur in prospective cohort studies and even randomized controlled trials ${ }^{2}$. This type of bias alludes on the fact that study participants recall information either inaccurately or incompletely. If distributed unevenly across study groups, it can affect the study's internal validity ${ }^{2-5}$. The distribution of recall errors determines the direction of bias ${ }^{4}$. Factors known to affect the reliability of recalled information include the state of memory $y^{6-8}$, duration of the retention interval ${ }^{9,10}$, patient demographics ${ }^{11}$ and the occurrence of events ${ }^{2,4}$.

Nowadays, self-reported pain intensity is used as an outcome measure in research more often than before ${ }^{11}$. Given the importance of self-reported pain scores in clinical research, its reliability is critical. Literature on the influence of recall bias on the assessment of pain scores in clinical studies is limited and contradictory $y^{8,12-19}$. Currently, it is unclear if recall error just results in inaccurate measurements or may also lead to a significantly altered outcome of retrospective studies on pain.

The main objective of the present study was to determine the influence of recall bias in surgical studies having pain intensity as the primary outcome. We compared retrospectively collected preoperative pain scores with prospectively collected pain scores to estimate the risk of recall error, recall bias, and erroneous conclusions due to recall bias. 


\section{METHODS}

\section{Setting}

The analysis was performed at Máxima Medical Centre (MMC), a 595-bed teaching hospital in Veldhoven/Eindhoven, the Netherlands. In recent years, a sub-department of general surgery ('SolviMáx') has specialized on the treatment of patients with chronic abdominal wall pain and groin pain syndromes. The number of evaluated patients has expanded over the years from around 250 in 2012 to 1045 in 2015. The present study did not require permission from a medical ethics committee since it involved evaluation of previously collected anonymous data.

\section{Study design}

\section{Study eligibility criteria}

We included data from all retrospective MMC cohort studies reporting results of surgical interventions for abdominal wall pain or groin pain. A study was considered eligible when preoperative self-reported pain scores were collected retrospectively using questionnaires or structured interviews (recalled data). These studies used retrospectively obtained pain scores because prospective pain scores were often missing from the patients' electronic hospital files. The original study databases were used for analysis. Studies that only used prospective data were excluded.

\section{Eligibility criteria for participants}

A subset of patients of the included studies was used for the current study. Only patients operated for chronic abdominal wall or groin pain in MMC were eligible. For individual patients, both preoperative and postoperative prospectively registered self-reported pain scores had to be retrievable from routine electronic patient records. We thereby excluded patients whose prospective pain scores were missing. Preoperative pain scores had to be collected retrospectively after surgery using questionnaires or structured interviews, as part of the study protocol. Furthermore, the prospectively and retrospectively applied pain scales had to be identical. Assembly of the treatment outcome (success or failure, table 9.1) had to be reproducible from the documented postoperative and preoperative pain scores. If patients participated in multiple studies, only data from the first study were used. Losses-to-follow up were excluded. 


\section{Data collection process}

Retrospectively obtained preoperative pain scores were extracted from the original study databases and were considered as 'potentially biased scores'. Preoperative and postoperative prospectively obtained pain scores were extracted from electronic patient records and were considered as 'unbiased scores'. Patient characteristics, type of pain treatment and effectiveness of pain surgery were collected from the original study databases. For the purpose of the present analysis, the original study databases were combined into a new database.

\section{Pain scales}

Three different pain scales were used in the selected studies. The numerical rating scale (NRS) instructs individuals to score pain on a 0 (no pain) to 10 (unbearable pain) scale. It is a commonly used one-dimensional pain scale that is easy to use for both clinical and research purposes ${ }^{20}$. The visual analogue scale (VAS) uses a horizontal line of $100 \mathrm{~mm}$ in length, the left end point $(0 \mathrm{~mm})$ representing absence of pain and the right end point $(100 \mathrm{~mm})$ indicating unbearable pain ${ }^{21,22}$. The patient is asked to place a mark on the line that corresponds to the intensity of the experienced pain. The verbal rating scale (VRS) consists of a five point (1-5) categorical Likert-like scale that uses commonly used words to describe pain (figure 9.1). Patients are asked to choose the words that best describe their pain ${ }^{23-25}$. It was assumed that the three pain scales corresponded in an absolute manner allowing pooling of results.
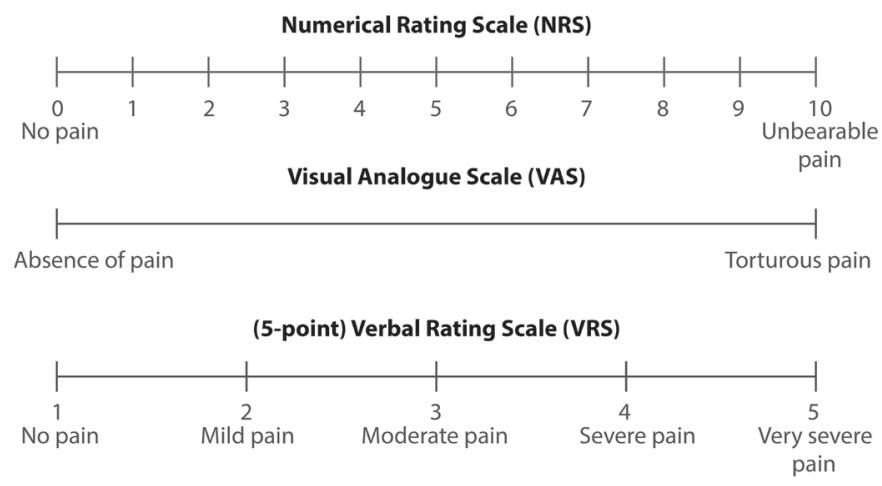

FIGURE 9.1:

Various pain scales that were used in the present analysis 


\section{Outcome definitions}

The primary outcome was the magnitude and direction of recall bias. Recall bias was defined as a systematic difference in overall treatment effect (i.e. study conclusion) between analyses using retrospective data and analyses using prospective data. Definitions of success or failure after a surgical intervention are described in table 9.1. A recall error was defined as a discrepancy between prospectively and retrospectively obtained, preoperative pain scores. Recall misclassification was defined as a recall error leading to misclassification of treatment outcome. A negative recall misclassification indicates that treatment success was falsely classified as failure based on retrospective scores, while prospective scores indicated a success. Conversely, a positive recall misclassification indicated that treatment failure was misclassified as treatment success.

\section{TABLE 9.1:}

Definitions of severe pain as defined by popular pain scores and its relation with outcome following surgical intervention

\begin{tabular}{lll}
\hline Pain score & Successful treatment & Failed treatment \\
\hline $\begin{array}{l}\text { Numerical Rating Scale (NRS) } \\
(0-10)\end{array}$ & $\begin{array}{l}\text { Postoperative NRS } \geq 50 \% \\
\text { reduction compared to } \\
\text { preoperative NRS }\end{array}$ & $\begin{array}{l}\text { Results not meeting the criteria } \\
\text { for successful treatment }\end{array}$ \\
$\begin{array}{l}\text { Visual Analogue Scale (VAS) } \\
(0-100)\end{array}$ & $\begin{array}{l}\text { Postoperative VAS reduction } \\
\text { of at least 50\% compared to } \\
\text { preoperative VAS }\end{array}$ & $\begin{array}{l}\text { Results not meeting the criteria } \\
\text { for successful treatment }\end{array}$ \\
$\begin{array}{l}\text { 5-point Verbal Rating Scale (VRS) } \\
(1-5)\end{array}$ & $\begin{array}{l}\text { A minimal 2-point reduction } \\
\text { using VRS at the postoperative } \\
\text { time point compared to } \\
\text { preoperative VRS }\end{array}$ & $\begin{array}{l}\text { Results not meeting the criteria } \\
\text { for successful treatment }\end{array}$ \\
\hline
\end{tabular}

\section{Stepwise approach}

A stepwise approach was used. First, accuracy of retrospectively obtained self-reported preoperative pain scores was assessed by comparing these values with prospective preoperative pain scores (recall error) within studies. Secondly, individual treatment outcomes were dichotomized as success or failure. Treatment outcome was classified as success or failure by using the retrospective or prospective preoperative scores for all individual patients, as compared to postoperative pain scores (table 9.1).

Misclassification of the treatment outcome due to the use of retrospective pain scores in individual patients was identified. The prevalence of recall misclassification within 
studies was calculated as the proportion of patients with misclassification of the treatment outcome. This was performed for positive and negative misclassifications, both separate as well as together. In addition, the net direction of the misclassification was presented as the difference in proportion of negative recall misclassification and positive recall misclassification. Thirdly, a meta-analysis was performed to investigate the difference in risk of misclassification between failures and successes. Significant differences point at factors leading to differential misclassification, resulting in an actual recall bias. Lastly, differences in recall bias between different pain scales (NRS, VAS, VRS) were analyzed.

\section{Statistical methods}

Data were analyzed using IBM SPSS Statistics 22 software (SPSS Inc., Chicago, Illinois, United States). Mean \pm SD or median [interquartile range; IQR] of the prospective and retrospective preoperative pain scores were calculated per study, as appropriate. Recall errors were assessed by comparing means (or medians) of these pain scores within studies. A paired student t-test (normal distribution) or Wilcoxon signed ranks test (non-normal distributed pain scores) were used to test statistical differences. Statistical significance was accepted at a two-sided $p$-value of $\leq 0.05$ and confirmed the presence of recall errors within studies. In addition, the absolute intraclass correlation coefficient (ICC) was calculated per study using the two way mixed model.

To assess the influence of treatment effect on the risk of recall bias due to retrospective relative to prospective collection of pain scores, odds ratios (ORs) and corresponding 95\%-confidence intervals (95\%CI) were calculated using Review Manager version 5.3 (The Cochrane Collaboration, London, UK). The number of negative and positive recall misclassifications and the total number of included successes and failures were entered so that an $\mathrm{OR}>1$ points at a higher risk of bias in failures than in successes. Hence an $O R>1$ indicates a bias towards a more positive treatment result with recalled pain scores than with prospective pain scores. ORs and 95\%CIs were depicted in a forest plot. A subgroup analysis was performed per pain scale. The random effects model was used for pooling the results. Statistical heterogeneity of studies regarding the recall bias was evaluated by Chi-square test and calculation of the inconsistency $\left(\mathrm{I}^{2}\right)$.

To explore the presence of selection bias in our analysis we compared baseline characteristics of included and excluded patients. Bivariate and continuous data were tested using the Chi-square test and independent t-test, respectively. 


\section{RESULTS}

\section{Studies and participants}

Seven studies on surgical treatment of patients with chronic abdominal wall or groin pain fulfilled the inclusion criteria ${ }^{23-29}$. Patient recruitment for these studies occurred between August 2000 and October 2013 (table 9.2). Four patients who did not undergo an intervention were excluded ${ }^{26}$. Sixty-six overlapping patients were excluded as well. Prospective, retrospective or postoperative pain scores were missing in 255 patients, leading to analysis of 313 (55\%) patients having complete pain data sets were analyzed in the present study (figure 9.2). Median patient follow-up was 21 months [IQR 12-30].

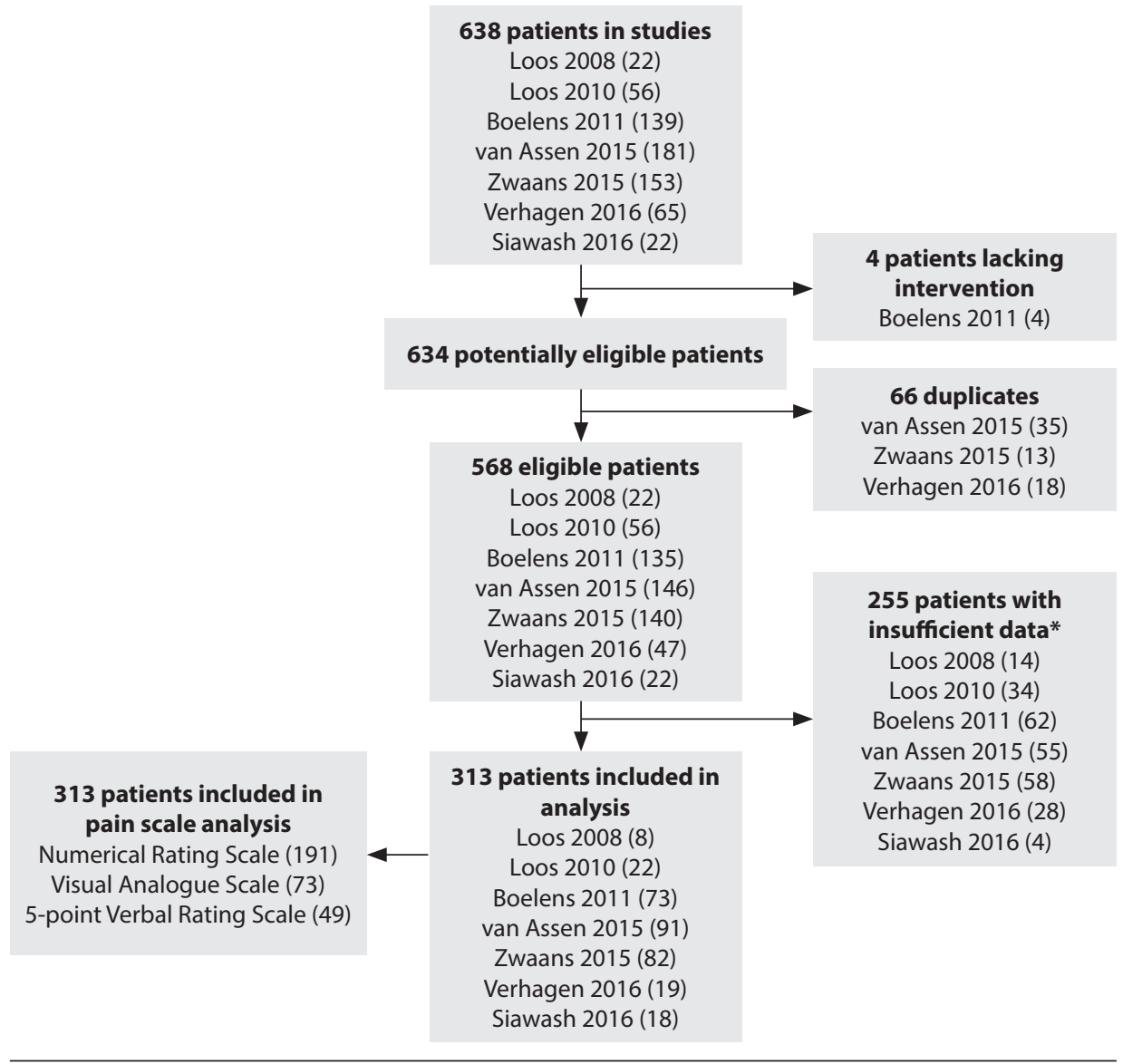

\section{FIGURE 9.2:}

Flowchart of the selection process. *Data were considered insufficient if the prospectively obtained, retrospectively obtained or postoperative pain score was missing. 

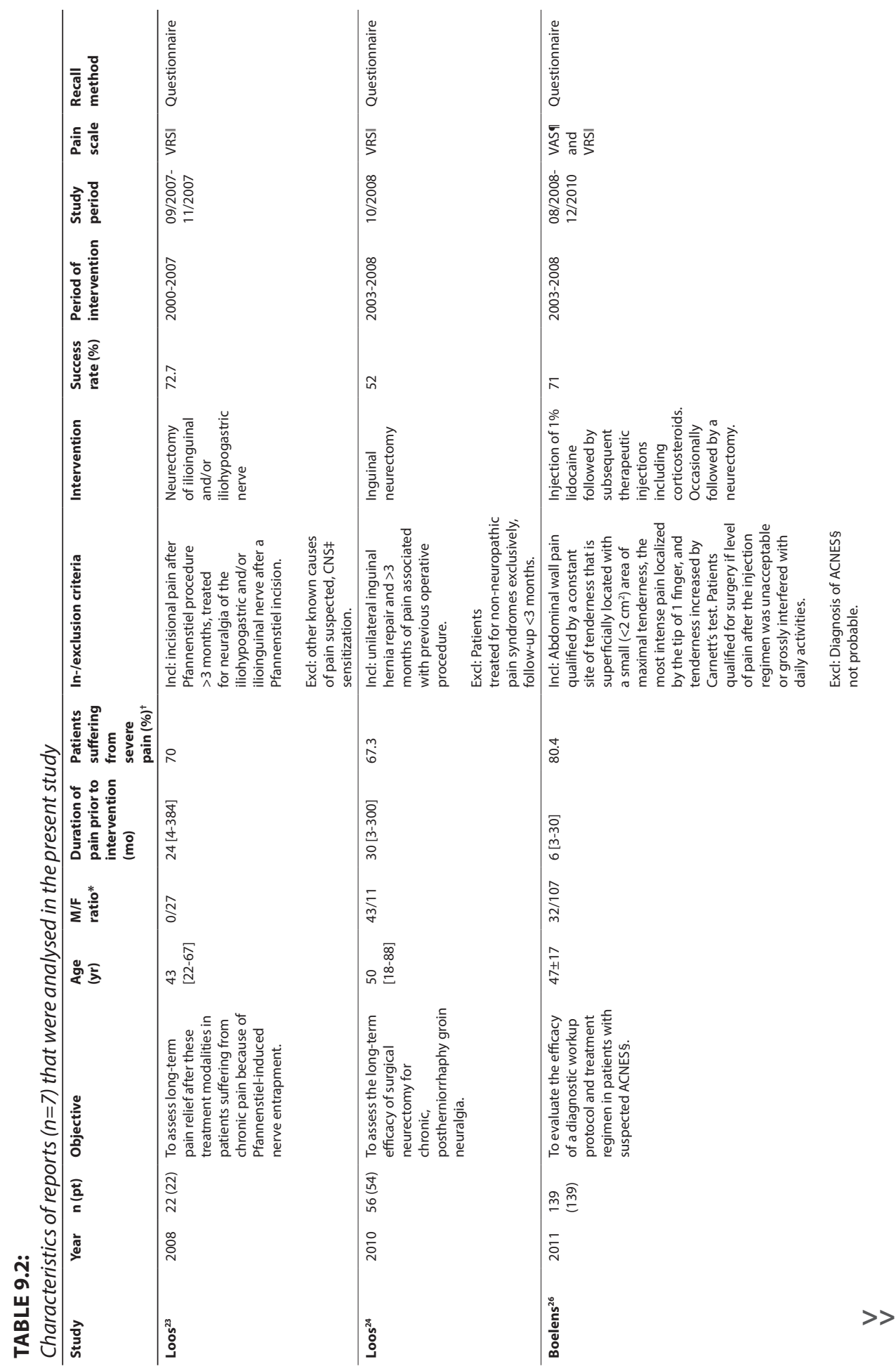


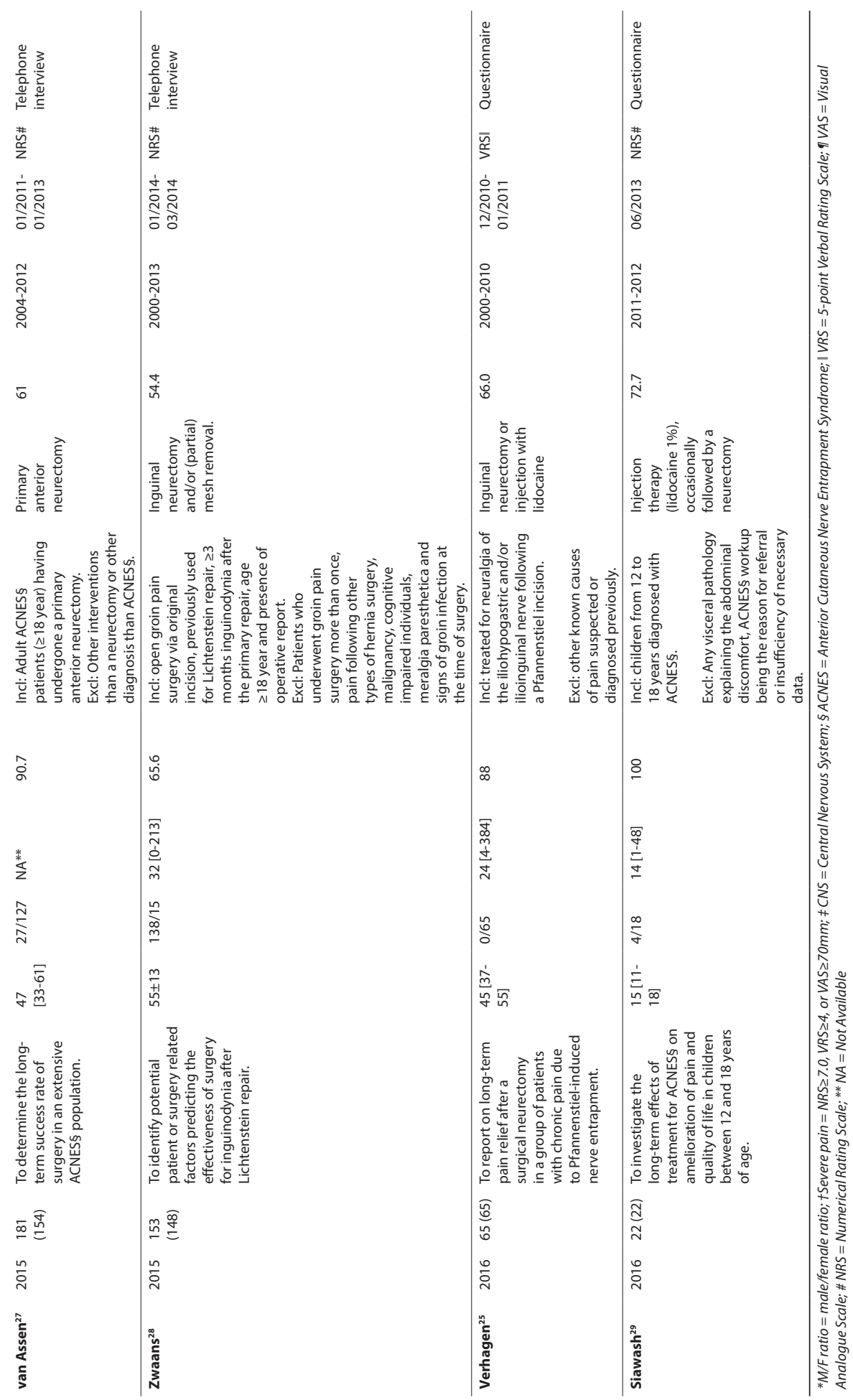

184 | Strategies for chronic inguinal pain 


\section{Main results}

Is a recall error present in studies?

Recall errors were present in all seven studies, but statistical significant differences between prospective and retrospective pain scores were found in only four studies (table 9.3). Agreement between preoperative prospective and retrospective pain scores within patients as expressed by ICC was fair in three studies whereas a moderate agreement was found in the four remaining studies.

\section{What is the actual misclassification of recall and is it differential?}

The overall prevalence of recall misclassification was 13.7\% [95\%CI: 10.3-18.0, range 3.3 to $36.4 \%$ ] (table 9.3). Figure 9.3 represents the percentage of recall misclassifications per study. The net amount of recall misclassification varied considerably (ranging from 1 to $33 \%)$ but all directed towards more positive misclassification if using retrospective pain scores.

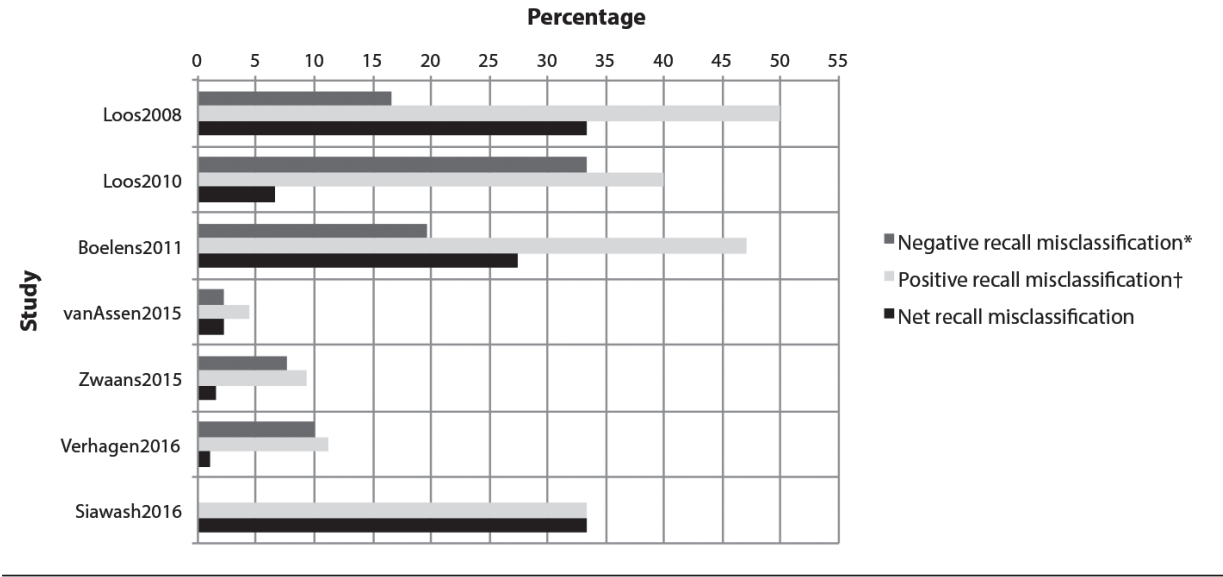

\section{FIGURE 9.3:}

Number of patients with positive and negative recall misclassifications by study.

*Negative values indicate negative recall misclassification (a shift from success to failure by the recall error). ${ }^{+}$Positive values indicate positive recall misclassification (shift from failure group (determined by prospective pain score) to the successful group (using retrospective pain score)). 


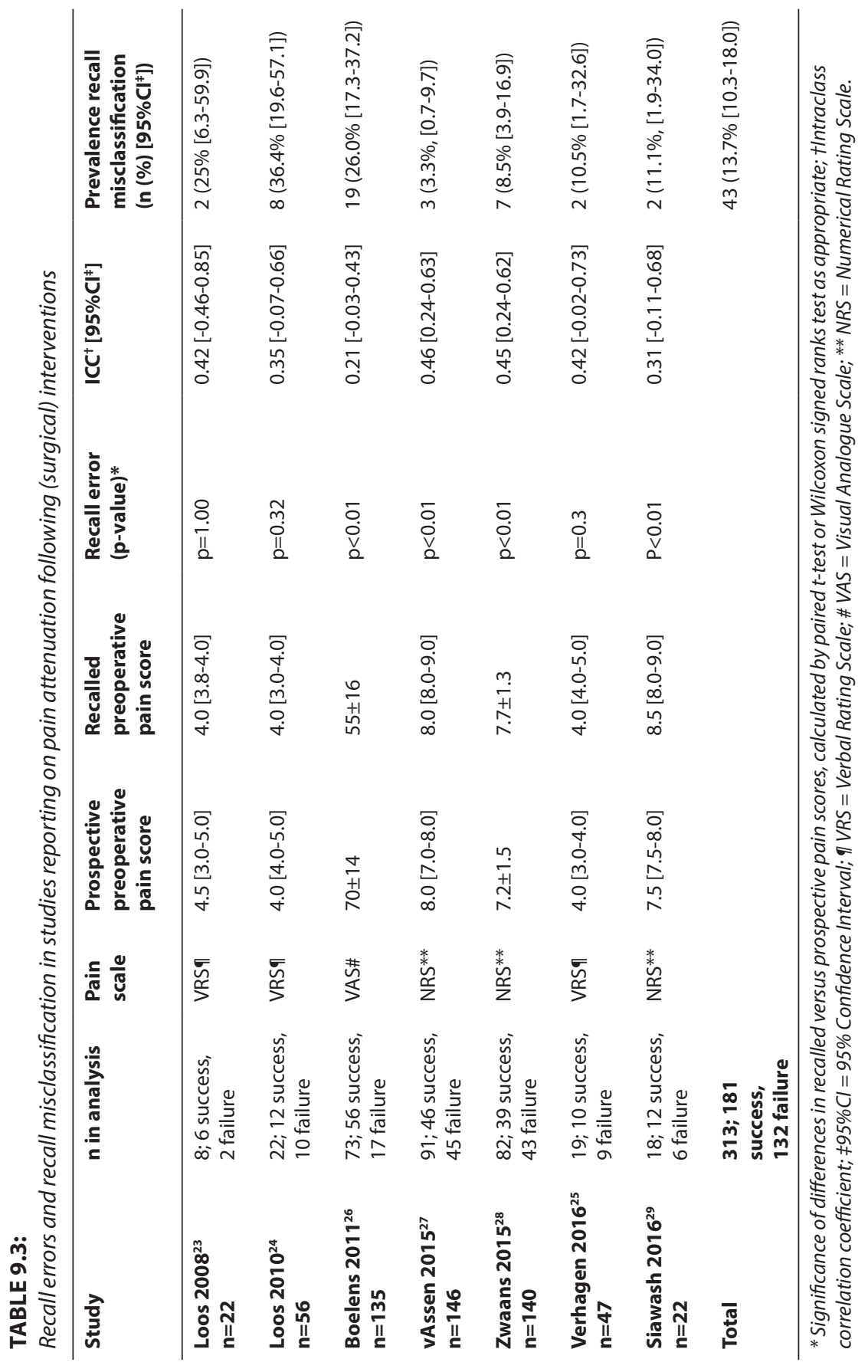


In general, patients failing treatment tended to recall preoperative NRS and VRS pain scores as significantly higher than they actually were as indicated by the prospectively obtained pain scores (NRS prospective median 7.5 [IQR 6.9-8.0] vs. recall median 8.0 [IQR 7.4-9.0]; VRS prospective median 4.0 [IQR 3.0-4.0] vs. VRS recall median 4.0 [IQR 4.0-4.5]). In contrast, the reverse was found regarding the VAS pain scale (VAS prospective mean $70.7 \pm 13.9$ vs. VAS recall mean $61.8 \pm 12.9$ ).

On the other hand, patients with a successful treatment outcome recorded lower preoperative pain scores if assessed retrospectively (VAS prospective mean $71.1 \pm 15.4$ vs. VAS recall mean 55.8 \pm 15.4 ; VRS prospective median 5.0 [IQR 4.0-5.0] vs. VRS recall median 4.0 [IQR 4.0-5.0]), with exception of the NRS pain scale (NRS prospective median 7.5 [IQR 7.0-8.0] vs. recall median 8.0 [IQR 7.5-9.0]).

\section{What is the actual recall bias in studies?}

ORs of recall misclassification in studies are presented in figure 9.4. An OR $>1$ indicates more positive recall misclassification (i.e. patients failing treatment if based on the prospective preoperative pain score but having a successful outcome if based on the retrospective preoperative pain score). On the contrary, an $\mathrm{OR}<1$ demonstrates more negative recall misclassification (i.e. patients successfully treated using prospective pre-operative pain score but having a unsuccessful outcome based on retrospective preoperative pain score). ORs varied considerably among the studies. The overall OR of 2.4 [95\%CI: 1.1-4.8] was significant, indicating predominance of positive misclassification over negative misclassification among studies. Therefore, an overall actual recall bias was present due to differential misclassification in successes and failures.

Heterogeneity as determined by the Chi-square test was absent $(\mathrm{p}=0.78)$. The $\mathrm{I}^{2}$ was $0 \%$, indicating no important inconsistencies between different studies (figure 9.4).

\section{Does recall bias differ per pain score?}

The prevalence of recall misclassification differed per type of pain score. Prevalence of recall misclassification in NRS was 6.3\% [95\%CI: 3.5-10.8], VAS 26.0\% [95\%CI: 17.3-37.2] and VRS 24.5\% [95\%CI: 14.5-38.2]. The OR of the recall misclassification also varied per pain score (figure 9.4) being 2.0 for NRS, [95\%CI: 0.6-6.7], 3.6 for VAS [95\%CI: 1.1-11.6], and 1.6 for the VRS pain scale [95\%CI: 0.4-6.3]. 


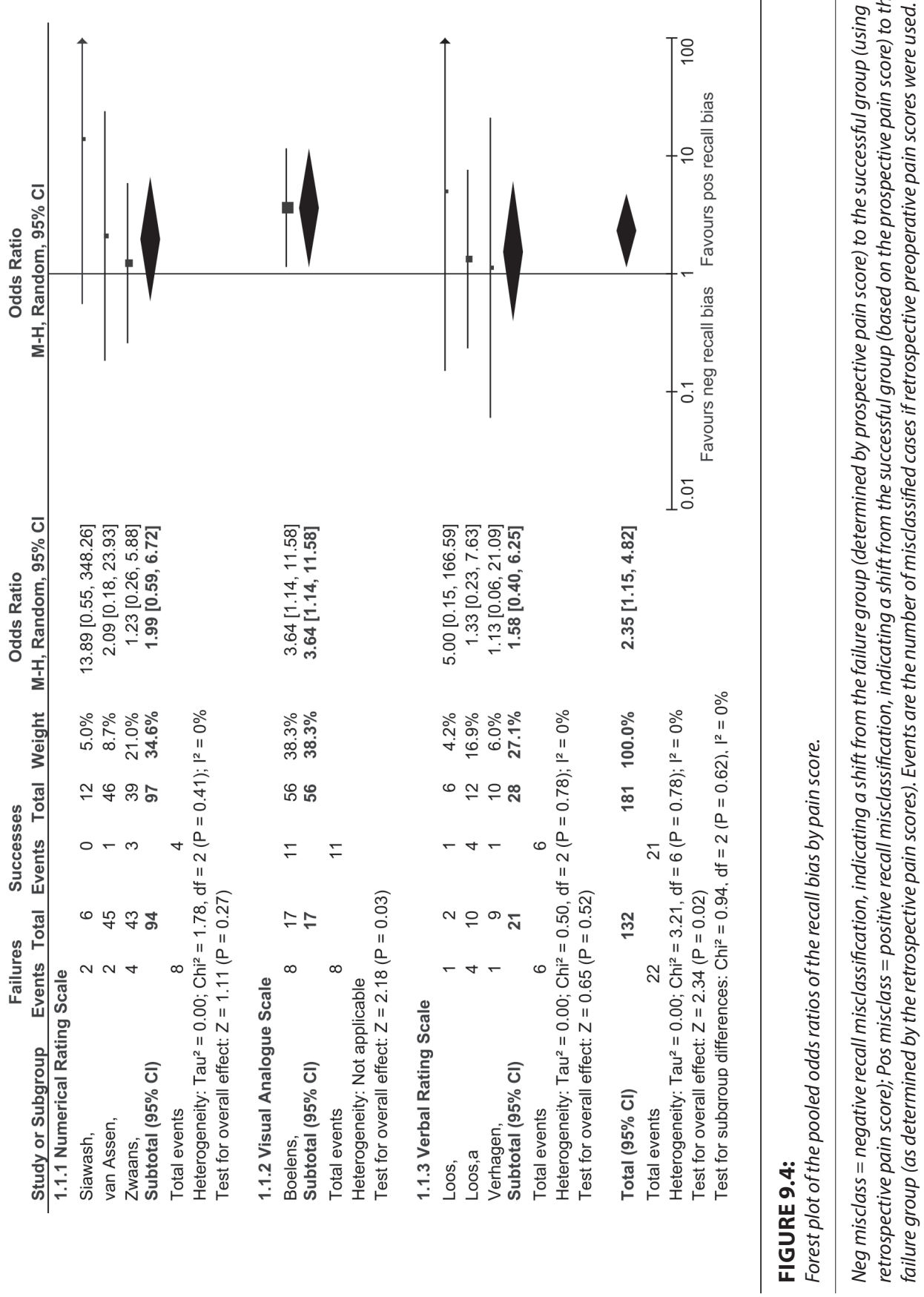




\section{Selection bias}

Characteristics of the population with incomplete data sets $(n=255)$ were similar to the population with sufficient data $(n=313$, table 9.4$)$ reducing the likelihood of selection bias in the present study.

\section{TABLE 9.4:}

Baseline characteristics of excluded (missing data sets) and included patients (complete sets of pain scores). Data are presented as means \pm standard deviation or ratios.

\begin{tabular}{lllll}
\hline Baseline Characteristic & $\begin{array}{l}\text { Excluded } \\
(\mathbf{n = 2 5 5 )}\end{array}$ & $\begin{array}{l}\text { Included } \\
(\mathbf{n = 3 1 3 )}\end{array}$ & p-value & Statistical test \\
\hline Sex ratio (male:female) & $108: 147$ & $118: 195$ & 0.26 & Pearson $\mathrm{X}^{2}$ test \\
Age (years) & $46.8 \pm 16.2$ & $47.0 \pm 17.7$ & 0.86 & Independent t-test \\
\% successful treatment outcome* & $58 \%$ & $58 \%$ & 0.95 & Pearson $\mathrm{X}^{2}$ test \\
\hline
\end{tabular}

* Treatment outcome ratio based on retrospectively obtained pain scores, as prospective pain scores were lacking.

\section{DISCUSSION}

The present study demonstrates that retrospectively collected pain scores of studies on efficacy of surgery for chronic groin pain result in erroneous measurement of pain intensities. It shows that misclassification due to recall errors affect both patients with successful surgery and patients with unsuccessful surgery, with an overall prevalence of $13.7 \%$. Positive recall misclassification is more likely to occur than negative recall misclassification with an overall pooled OR of 2.4 [95\%CI: 1.2-4.8]. Patients with an unsuccessful outcome recalled their preoperative pain scores as being higher than they actually were as indicated by preoperatively obtained pain scores. Conversely, patients with successful surgery demonstrated lower pain intensities when recalled. It may be concluded that using recalled pain scores has a significant impact on the measurement of surgical outcomes of patients suffering from abdominal wall or groin pain, depending on the success rate. Hence, recall bias does indeed exist in this patient population.

The present study demonstrated significant recall bias if relying on retrospectively acquired pain scores. A schematic version illustrating how the present results should be 
interpreted in the context of other studies using recalled pain scores is depicted in figure 9.5. In the first example, a hypothetical retrospective study is performed using recalled pre-intervention pain scores. The hypothetical study included 100 patients and assumes that the intervention is successful in 80 patients (80\%). As demonstrated by the present study, recall misclassification affected $13.7 \%$ of patients (table 9.3). For the purpose of clarity, let us assume that in $15 \%$ of the patients treatment effect is misclassified due to recall. Recall misclassification was about twice more likely in failures than in successfully treated patients (OR 2.35; figure 9.4). As there are fewer failures $(n=20)$ than successes $(n=80)$, the absolute number of misclassified patients in the success group is higher. As a consequence, 5 of the 15 hypothetical misclassified patients were actually failures (based on prospective pre-intervention pain scores). The other 10 misclassified patients were actually successes. The net number of misclassifications is 5 patients (10 minus 5). Following this line of thought, the percentage of successfully treated patients decreased from 80 to $75 \%$ and the number of failures increased by $5 \%$.

A second example is illustrated in figure 9.5. Since the number of successes is now lower, the absolute number of misclassified patients in the successful group is lower. This leads to a $5 \%$ success overestimation in this hypothetical example if retrospective pre-intervention data are used. Using a similar calculation based on true data from the present study indicated that the net recall bias is nullified if the success rate is $67 \%$ (figure 9.6). As a consequence, the net direction will go towards an underestimation of the treatment effect in highly successful treatment (i.e. $>67 \%$ ). Conversely, it will go towards an overestimation of the treatment effect in a less successful treatment $(<67 \%)$. 


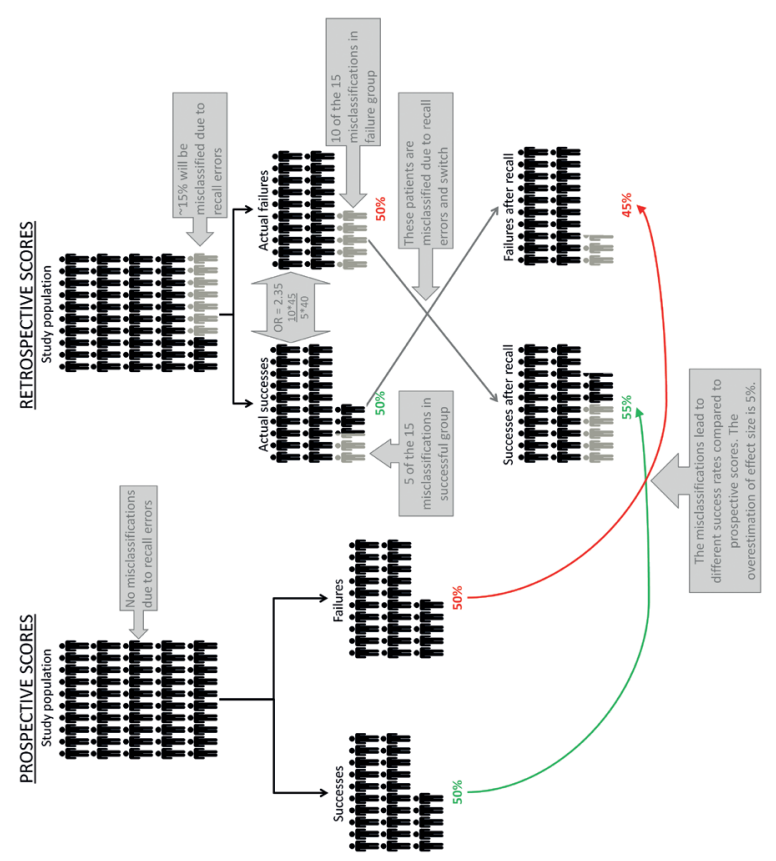

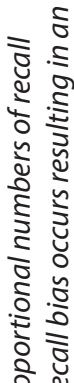

जิ जे

ิำ

인

$\frac{5}{\frac{1}{2}}$

气

永范

$\frac{1}{3} \frac{1}{2}$

Чิ

$\stackrel{5}{\approx}$

$\subseteq$.

ฟ

ฆ อิ

ข. ลิ

ปั ธิ

एँ

ปั อิ

के ज

ত্ড

¿ Uِ

ปี

$\frac{2}{2} \frac{0}{2}$

㲾 $\frac{0}{0}$

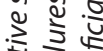

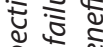

ติ.ई อ

은

ข

ị. $\leqq$ ত

ค่ $\tilde{~}$

山.

는 


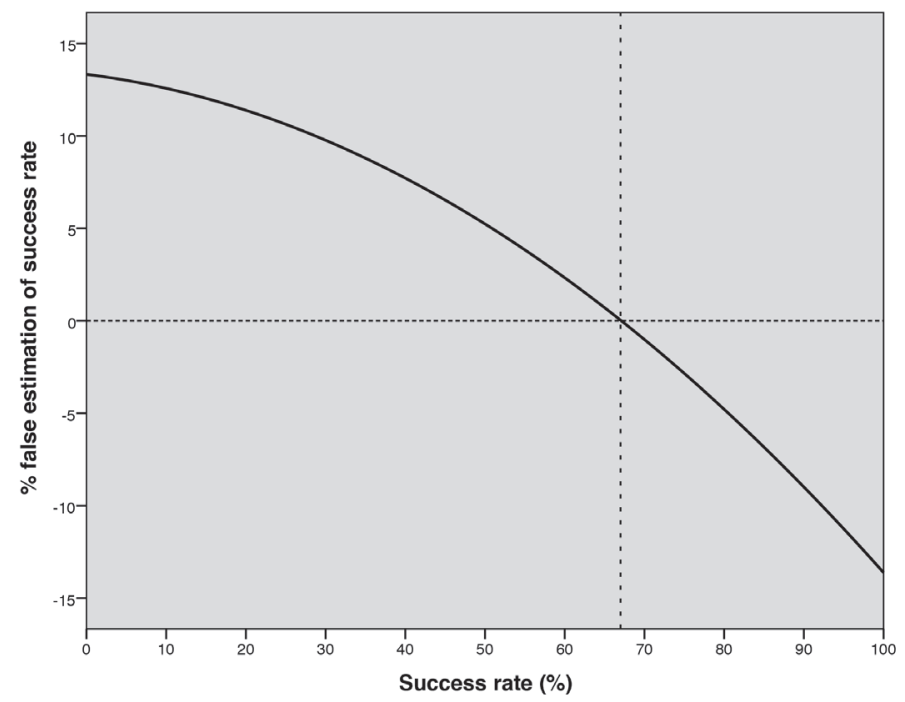

FIGURE 9.6:

Percentage of false estimation, based on the retrospective pain scores in relation to the actual success rate, as based on prospective pain scores.

Previous studies on total knee arthroplasty ${ }^{17}$, total hip replacement ${ }^{16}$ or treatment for lower back pain ${ }^{19}$ reported significantly higher pain levels if using recalled data. Additional literature on recall bias also confirms our finding that pain is often remembered as more intense by patients suffering from pain after treatment whereas pain intensity is underestimated after success ${ }^{12,30-32}$. Others suggested that errors in recalling pain intensity are generally non-differential ${ }^{8,10,13,15,33}$.

Most researchers would argue that current state of mood influences pain recollection $^{7-9,11,12,32,34,35}$. A clear example illustrating this theory is the recall of pain intensity in a postnatal stage ${ }^{36}$. Women who just gave birth underrate previously experienced pain during labor due to an overwhelming feeling of happiness caused by carrying their healthy newborn. In other words, patients become accustomed to improvements in their condition, a term that is referred to as 'satisfaction treadmill'6,37. Results of the present study also demonstrate that pain free patients do (probably unintentionally) underestimate their preoperative pain, possibly as a result of the positive emotions experienced during recall. A similar theory may, vice versa, hold true for a failure group. Their negative emotions will modulate memory processing and 
consequently, recall of pain in the past ${ }^{35}$. It may be concluded that recall pain intensities are likely congruent with pain, emotions and interference of daily activities of the pain at the time of recall. These phenomena may lead to higher and lower recalled preoperative pain scores in failure and successes, respectively.

\section{Potential study limitations}

Our study has several potential limitations. By pooling results of different pain scales we assumed that pain scales correspond to one another in an absolute manner. The present study hypothesized that a ' 6 ' on the NRS was similar to a ' 3 ' on the VRS and a ' 60 ' on the VAS scale. There was however no statistical heterogeneity which ensures that pooling was valid. A possible limitation is the fact that the analysis of recall error is likely to be underpowered, since some of the seven studies included less than 20 eligible patients. However, the main issue is whether prospectively obtained pain scores differ from retrospectively obtained pain scores. Independently of the statistical analysis used to assess this issue, the prospective and recalled pain scores indicate a significant difference.

Selection bias may have been created as populations with incomplete data were excluded. Since the basic characteristics of included and excluded patients were similar selection bias is less likely to play a role. Publication bias was avoided since all eligible MMC studies were included irrespective of publication status.

The present study demonstrates that recall bias varied between pain scales. VAS seemed most susceptible to recall error but this observation relied on one single study. Therefore, no firm conclusions can be drawn and further research is required.

\section{CONCLUSIONS}

Surgery outcomes in one in seven patients undergoing remedial surgery are misclassified on the basis of retrospectively obtained preoperative pain scores (success instead of failure, or vice versa). Misclassification is more likely in unsuccessful surgery than successful surgery. Therefore, the estimated effect size in studies using recalled preoperative pain scores depend on the actual success rate. Success rates exceeding $67 \%$ are underestimated, whereas effect sizes are overestimated when success rates are below $67 \%$. Detailed pain scales seem to be more susceptible for recall errors, but this issue needs further investigation. 


\section{REFERENCES}

1. Miettinen OS. Principles of Epidemiologic Research. Cambridge: School of Public Health, Harvard University, 1975.

2. Hassan E. Recall Bias can be a Threat to Retrospective and Prospective Research Designs. The Internet Journal of Epidemiology 2005;3(2).

3. Last JM. A Dictionary of Epidemiology. $4^{\text {th }}$ edition ed. Oxford: Oxford University Press, 2000.

4. Lippman A, Mackenzie SG. What is "recall bias" and does it exist? Prog Clin Biol Res 1985;163C:205-9.

5. Sedgwick P. What is recall bias? BMJ 2012;344.

6. Gendreau M, Hufford MR, Stone AA. Measuring clinical pain in chronic widespread pain: selected methodological issues. Best Pract Res Clin Rheumatol 2003;17(4):575-92.

7. Redelmeier DA, Kahneman D. Patients' memories of painful medical treatments: real-time and retrospective evaluations of two minimally invasive procedures. Pain 1996;66(1):3-8.

8. Salovey P, Smith AF, Turk DC, et al. The accuracy of memory for pain: Not so bad most of the time. APS Journal 1993;2(3):184-91.

9. Eich E. On the accuracy of memory for pain. APS Journal 1993;2(3):192-94.

10. Middel B, Goudriaan H, de Greef M, et al. Recall bias did not affect perceived magnitude of change in health-related functional status. J Clin Epidemiol 2006;59(5):503-11.

11. Williams DA, Park KM, Ambrose KR, et al. Assessor status influences pain recall. J Pain 2007;8(4):343-8.

12. Eich E, Reeves JL, Jaeger B, et al. Memory for pain: relation between past and present pain intensity. Pain 1985;23(4):375-80.

13. Rofe Y, Algom D. Accuracy of remembering postdelivery pain. Percept Mot Skills 1985;60(1):99-105.

14. Raphael K. Recall bias: a proposal for assessment and control. Int J Epidemiol 1987;16(2):16770.

15. Wright J, Morley S. Autobiographical memory and chronic pain. Br J Clin Psychol 1995;34 ( Pt 2):255-65.

16. Mancuso CA, Charlson ME. Does recollection error threaten the validity of cross-sectional studies of effectiveness? Med Care 1995;33(4 Suppl):AS77-88. 
17. Lingard EA, Wright EA, Sledge CB. Pitfalls of using patient recall to derive preoperative status in outcome studies of total knee arthroplasty. J Bone Joint Surg Am 2001;83-A(8):114956.

18. Stone AA, Broderick JE, Shiffman SS, et al. Understanding recall of weekly pain from a momentary assessment perspective: absolute agreement, between- and within-person consistency, and judged change in weekly pain. Pain 2004;107(1-2):61-9.

19. Pellise F, Vidal X, Hernandez A, et al. Reliability of retrospective clinical data to evaluate the effectiveness of lumbar fusion in chronic low back pain. Spine 2005;30(3):365-8.

20. Ho K, Spence J, Murphy MF. Review of pain-measurement tools. Ann Emerg Med 1996;27(4):427-32.

21. Loos MJ, Houterman S, Scheltinga MR, et al. Evaluating postherniorrhaphy groin pain: Visual Analogue or Verbal Rating Scale? Hernia 2008;12(2):147-51.

22. Price DD, McGrath PA, Rafii A, et al. The validation of visual analogue scales as ratio scale measures for chronic and experimental pain. Pain 1983;17(1):45-56.

23. Loos MJ, Scheltinga MR, Roumen RM. Surgical management of inguinal neuralgia after a low transverse Pfannenstiel incision. Ann Surg 2008;248(5):880-5.

24. Loos MJ, Scheltinga MR, Roumen RM. Tailored neurectomy for treatment of postherniorrhaphy inguinal neuralgia. Surgery 2010;147(2):275-81.

25. Verhagen T, Roumen RM, Loos MJ, et al. Surgical neurectomy for resistive chronic postPfannenstiel pain syndrome is effective. Submitted 2016.

26. Boelens OB, Scheltinga MR, Houterman S, et al. Management of anterior cutaneous nerve entrapment syndrome in a cohort of 139 patients. Ann Surg 2011;254(6):1054-8.

27. van Assen T, Boelens OB, van Eerten PV, et al. Long-term success rates after an anterior neurectomy in patients with an abdominal cutaneous nerve entrapment syndrome. Surgery 2015;157(1):137-43.

28. Zwaans WA, Verhagen T, Roumen RM, et al. Factors Determining Outcome After Surgery for Chronic Groin Pain Following a Lichtenstein Hernia Repair. World J Surg 2015;39(11):265262.

29. Siawash M, Mol FM, Perquin CW, et al. Management of anterior cutaneous nerve entrapment syndrome (ACNES) in adolescents. Submitted 2016.

30. Choi BC, Noseworthy AL. Classification, direction, and prevention of bias in epidemiologic research. J Occup Med 1992;34(3):265-71. 
31. Jamison RN, Sbrocco T, Parris WC. The influence of physical and psychosocial factors on accuracy of memory for pain in chronic pain patients. Pain 1989;37(3):289-94.

32. Tasmuth T, Estlanderb AM, Kalso E. Effect of present pain and mood on the memory of past postoperative pain in women treated surgically for breast cancer. Pain 1996;68(2-3):343-7.

33. Hunter M, Philips C, Rachman S. Memory for pain. Pain 1979;6(1):35-46.

34. Bower GH. Mood and memory. Am Psychol 1981;36(2):129-48.

35. Gedney JJ, Logan H. Pain related recall predicts future pain report. Pain 2006;121(1-2):6976.

36. Norvell KT, Gaston-Johansson F, Fridh G. Remembrance of labor pain: how valid are retrospective pain measurements? Pain 1987;31(1):77-86.

37. Kahneman D. Objective happiness. In: Kahneman D, Deiner, E., Schwarz, N., ed. Well-being: the Foundations of Hedonic Psychology. New York City (NY), USA: Russell Sage Foundation, 1999:3-25. 



\section{CHAPTER 10}

Summary, conclusions and general discussion 


\section{SUMMARY}

\section{Prevention of chronic postoperative inguinal pain}

Inguinal hernia recurrence rates have dropped drastically since the introduction of prosthetic meshes in $1958^{1}$. However, the search for the optimal mesh is ongoing as the use of body foreign material is potentially associated with chronic ( $>3$ months) postoperative inguinal pain (CPIP) in some patients. Nowadays, CPIP is the most refractory complication following inguinal hernia repair and poses a major health issue. Strategies to reduce CPIP are currently extensively studied but "prevention is better than cure" (Desiderius Erasmus, 1466-1536).

One strategy to prevent CPIP focuses on the exploration of alternative type of meshes. A self-gripping mesh was designed that adheres to tissue surrounding the groin wall defect using vicryl micro-hooks $\left(\text { Progrip }^{\mathrm{Ts}}\right)^{2}$. This semi-resorbable and lighter mesh omitted the need for suture fixation and was claimed to potentially prevent the onset of CPIP. A trial reporting on postoperative pain levels compared a conventional polypropylene sutured mesh and this self-gripping Progrip ${ }^{\text {Tx }}$ mesh (chapter 2). No significant difference in pain intensity was demonstrated after one year. It was concluded that the ProGrip ${ }^{\text {Tx }}$ mesh may attenuate early postoperative pain but the effect on CPIP was absent.

As the assessment of realistic CPIP rates need a longer than one year follow-up, threeyear results of this double-blinded randomized controlled trial (RCT) were evaluated in chapter 3. Additional outcomes were hernia recurrences, characteristics of neuropathic and nociceptive pain as well as sexual functioning. Three years following insertion of a selfgripping Progrip ${ }^{\mathrm{Ts}}$ mesh or a sutured polypropylene mesh for an open primary inguinal hernia repair, groin pain was minimal although rates of altered groin skin sensations and foreign body feeling were found quite common and even higher than after one year. However, the patient group receiving the Progrip ${ }^{\text {Tx }}$ had a significantly higher recurrence rate. It may be concluded that this type of self-gripping mesh does not prevent CPIP and is not recommended as the standard approach for primary open inguinal hernia repair.

\section{Surgical treatment options for CPIP}

The majority of both conservative and invasive therapies focuses on treatment of the inguinal nerves (ilioinguinal, iliohypogastric or genitofemoral nerve) as CPIP often is deemed of neuropathic (nerve-related) origin. Removal of a portion of the 
nerve (neurectomy) of one or more inguinal nerves is frequently considered. However, a selective group of patients does not benefit from neither conservative nor surgical treatment. These patients may have a predominantly nociceptive or inflammatory type of inguinal pain that is probably due to presence of the mesh. However, precise descriptions of clinical characteristics of a typical mesh-related pain are currently lacking. In chapter 4, the characteristics of mesh-related CPIP are described to serve as a general tool of guidance for (hernia) specialists and other physicians. A pure mesh-related inguinal pain is suggested by a 'foreign body feeling'. The pain intensifies during hip flexion (e.g. car driving) and is attenuated following hip extension or supine position. Palpation is painful along the inguinal ligament whereas neuropathic characteristics (hyperpathic skin, trigger points) are lacking.

A retrospective study analyzing 74 consecutive patients undergoing open meshectomy with or without selective neurectomy for CPIP following Lichtenstein hernia repair was performed (chapter 4). Mesh removal either or not combined with tailored neurectomy was beneficial in two of three patients (median follow-up 18 months). Success rates of a meshectomy alone $(63 \%)$ or combined with a neurectomy $(64 \%)$ did not differ. In one of 15 patients, the inguinal hernia recurred. A literature search was performed, aimed at identifying studies on mesh removal (chapter 4). Based on a favourable outcome with acceptable complication rates and supported by encouraging data from previous studies, mesh removal combined with tailored inguinal neurectomy should be considered in CPIP patients unresponsive to other treatments including neurectomies.

Evidence suggests that endoscopic inguinal hernia repairs (e.g. total extraperitoneal procedure, TEP or a transabdominal preperitoneal procedure, TAPP) have a reduced chance on CPIP when compared to open surgery ${ }^{3-5}$. Moreover, these techniques are costeffective and associated with shorter recovery times ${ }^{6}$. Therefore, a growing number of (Western) surgeons prefer endoscopic repair techniques over conventional open repairs. Mesh-related CPIP after endoscopic repair is less prevalent than following open repair, but as the endoscopic techniques popularize, the absolute numbers of CPIP patients following TEP or TAPP will likely rise in the next years. An exciting new remedial surgical technique is to laparoscopically remove the previously implanted mesh that is described in chapter 5. Feasibility, safety and efficacy were studied in a case series of 14 patients. Considering no conversions to open surgery and one intraoperatively corrected bladder laceration without any permanent harm, the method is considered feasible 
and safe. Median operating time was 103 minutes. Median pain scores dropped from 8 to 4 (11-point Numerical Rating Scale, NRS). Ten months after surgery, satisfaction was excellent or good in $71 \%$. Based on this proof-of-concept study, laparoscopic mesh removal is judged as a feasible method to treat mesh-related pain after endoscopic preperitoneal mesh-based repair.

During remedial surgeries, some remarkable findings may be encountered. The Lichtenstein procedure is gold standard of hernia surgery in developing countries and also frequently performed in the Western world ${ }^{6}$. One of the advantages of the technique is a thoroughly documented sequence of surgical steps ${ }^{7}$. However, pitfalls are potentially looming as illustrated by the intraoperative finding of a spermatic cord that was divided by the mesh (Case I). Following a tailored neurectomy of the genital branch of the genitofemoral nerve, the patient was pain free.

An also surprising intraoperative finding was the encasement of an appendix vermiformis following an inguinal hernia plug repair according to Rutkow and Robbins (Case II). Prior to operation, the patient complained of tightness in the right groin area, painful urge of miction and long-lasting nausea with abdominal discomfort. After removal of the appendix that was tightly attached to the groin plug, the patient became pain free and his long-lasting nausea and abdominal discomfort disappeared as well. Surgeons should be aware of the potential migration of meshes and a potential phenomenon of referred visceral pain, which may be caused by the inserted mesh.

\section{Optimizing surgical treatment for CPIP}

The current surgical procedures for relief of CPIP are safe and reasonably effective. Factors determining success following remedial surgery, however, are unknown. A retrospective study was performed to identify potential patient or surgery related factors predicting the efficacy of remedial surgery for CPIP following a Lichtenstein repair (chapter 6). A total of 136 groin pain operations were available for analysis. A multivariate logistic regression analysis suggested that pain reduction after remedial surgery is more successful if the procedure is performed under spinal anaesthesia when compared to general anaesthesia (odds ratio, OR 4.0). When a meshoma was identified and removed, success rates are also optimized (OR 5.3). In contrast, preoperative opioids use was associated with a less favourable outcome (OR 0.4). These associations came somewhat as a surprise. It was questioned whether these results were reproducible in a 
larger and more diverse group of patients with inguinal pain. Especially the finding of a potential beneficial effect of spinal anaesthesia was remarkable as type of anaesthesia is easily modifiable.

A second study was performed, aiming at investigating whether spinal anaesthesia is indeed preferable over general anaesthesia in patients undergoing remedial surgery for various types of chronic inguinodynia (chapter 7). The study comprised 339 inguinal pain patients following a range of primary surgeries, varying from different types of inguinal and incisional herniorrhaphy to abdominoplasty and caesarean sections. The most frequently performed remedial surgery was an isolated neurectomy $(n=235)$, whereas spinal anaesthesia was applied in $41 \%$ of all patients. Again, spinal anaesthesia showed a significant positive association with success (OR 1.9), a finding that was most evident in patients who underwent a neurectomy (OR 2.3). However, following a sensitivity analysis, it was concluded that the effect size was possibly determined by statistical methods. Therefore, findings should be interpreted with caution.

Following this line of thought, a RCT was designed to study the effect of type of anaesthesia (spinal or general) on pain relief following remedial surgery for inguinal pain, circumventing the types of bias associated with retrospective studies (chapter 8). One-hundred-ninety adult patients who suffer from unacceptable chronic inguinal pain will be included and randomized to spinal or general anaesthesia. Primary outcome is effect of type of anaesthesia on pain relief with a follow-up period of one year. Secondary outcomes include patient satisfaction, quality of life, use of analgesics and (in)direct medical costs. Since potential confounding factors (e.g. perioperative administration of other medications, communication, surgical stress) are not controlled, a possible beneficial effect cannot be attributed to just one particular factor. Therefore, a potential effect should be considered as the end result of the total setting of type of anaesthesia and associated factors. The RCT was intentionally designed in this manner to generate clinically relevant conclusions that can be directly implemented in clinical practice. Patient enrolment started January 2016 and recruitment is still going. Expected trial deadline is December 2019.

\section{Recall bias}

Most pain studies primarily focus on self-reported pain intensities as an outcome measure. However, recall bias (a subtype of information bias) commonly arises in 
retrospective studies and may also occur in prospective cohort studies, and even in $\mathrm{RCTs}^{8}$. This type of bias alludes on the fact that patients remember the information either inaccurately or incompletely. If distributed unevenly across study groups, this phenomenon can affect the study's internal validity ${ }^{8-11}$. Given the importance of selfreported pain scores in clinical research, its reliability is critical. A study was performed to determine the influence of recall bias in surgical studies having pain intensity as the primary outcome (chapter 9).

The study results demonstrated that retrospectively collected pain scores of studies on efficacy of surgery for chronic abdominal wall and groin pain result in erroneous measurement of pain intensities. It was shown that misclassification due to recall errors affect both patients with successful surgery as well as unsuccessful surgery, with an overall prevalence of $13.7 \%$. Positive recall misclassification is more likely to occur than negative recall misclassification with an overall pooled OR of 2.4. Patients with an unsuccessful outcome recalled their preoperative pain scores as being higher than they actually were, as indicated by preoperatively obtained pain scores. Conversely, patients with successful surgery demonstrated lower pain intensities when recalled. It is concluded that the use of recalled pain scores does have a significant impact on the measurement of surgical outcomes of patients suffering from abdominal wall or groin pain, depending on the success rate.

\section{CONCLUSIONS}

1. Self-gripping meshes for open inguinal hernia repair are not superior compared to conventional, sutured meshes regarding CPIP but are associated with a higher recurrence rate after three years.

2. Altered groin skin sensation and foreign body feeling are quite common following open inguinal hernia repair and may become more prevalent over time.

3. Mesh-related groin pain is characterized by a 'foreign body feeling', provoked by hip flexion (e.g. car driving), attenuated in supine position and is evoked by palpation along the entire inguinal ligament whereas neuropathic characteristics are lacking. 
4. Open mesh removal is effective in two of three patients with mesh-related CPIP following open inguinal hernia repair.

5. Laparoscopic mesh removal is a safe, feasible and effective therapy in most patients suffering from mesh-related CPIP following endoscopic mesh insertion.

6. Pain reduction after remedial surgery for CPIP is possibly more profound if performed under spinal anaesthesia when compared to general anaesthesia.

7. If a meshoma is identified during remedial surgery for CPIP, removal is advised as the chance on a successful outcome is increased whereas further erosion and potential invasion of mesh into neighbouring viscera is prevented.

8. Recalled pain scores have a significant impact on the effect sizes of retrospective studies. The extent of the recall bias depends on the success rate of the intervention.

\section{DISCUSSION}

\section{Postherniorrhaphy pain over time}

The International Association for the Study of Pain (ISAP) defines CPIP as 'pain beyond three months after inguinal hernia surgery'12. Some have suggested to extent this period to six months ${ }^{13}$. Most cases of CPIP are assumed to diminish over time or to resolve spontaneously, without any contribution of the previously performed surgical procedure $^{14-16}$. Groin pain following hernia repair in the studies reported in this thesis stabilizes after one year postoperatively (chapter 2 and chapter 3 ). Moreover, rates of CPIP were notably low and not different in both groups of patients with a self gripping or sutured mesh at that time point (chapter 3). One retrospective study performed by others also demonstrated similar pain rates at three years following self-gripping and standard meshes ${ }^{17}$. Another study postulated that postoperative pain will continue to drop below $2 \%$ after five years ${ }^{18}$. However, results of the study reported in chapter 3 demonstrated that rates of CPIP stabilized at 12 months in both groups. As a consequence, defining CPIP as pain beyond 12 months following inguinal hernia repair is possibly more appropriate to compensate for the number of patients in whom pain will fade out. However, it is unclear whether pain after one year postoperatively is still due to the surgery itself (i.e. nerve damage, mechanical irritation of the mesh) or if the presence of other (psychosocial) factors contribute to an increased risk on developing chronic pain. 


\section{Sequelae of mesh-based inflammation}

One factor that may contribute to CPIP is related to the body's inflammatory reaction to inserted mesh. Locoregional inflammation is assumed critical for strengthening the hernia repair as formation of fibrosis is correlated to the intensity of inflammation ${ }^{19,20}$. If mesh is removed owing to a hernia recurrence, lower collagen levels are found when compared to situations requiring mesh removal for other causes ${ }^{21}$. However, the down side is that excessive inflammation occasionally leads to unfavourable effects such as erosion or invasion into the abdominal cavity as demonstrated in this thesis (Case II).

One may question whether mesh-related tissue responses are self-limiting. Meshes that were explanted after three years were still demonstrating active foreign body reactions ${ }^{21}$. Moreover, mesh-based inflammatory reactions to adjacent tissues such as an appendix vermiformis have been described one and a half year following primary hernia repair ${ }^{19}$. These findings support the idea that inflammation may go on for years. Other structures neighbouring the mesh may also be affected. For instance, if inguinal nerves respond to the inflammatory process, it could be possible that somatosensory disturbances will manifest over time. This hypothesis may possibly explain the increase in groin skin sensory dysfunctions that developed between one to three years following hernia repair as shown in chapter 3.

Inserted meshes may not only lead to a low grade chronic inflammatory reaction, but may also exert mechanical pressure or irritation possibly contributing to pain. The form of inserted mesh may change as creasing and folding may occur. This slow process increases mechanical pressure and may evoke pain during hip flexion and pain relief in supine position. Other novel pain characteristics following open inguinal hernia repair were reported in chapter 3. A frequently reported nociceptive characteristic is a 'foreign body feeling. Patients report that something is constantly bothering them as if a 'wrinkled handkerchief is sitting in their pocket'. The incidence of this symptom was higher after one year of follow-up and characterizes mesh-related pain, as described by the study on open meshectomies (chapter 4).

\section{Mesh-related pain}

A foreign body feeling may herald mesh-related pain. The finding that most meshrelated pain develops over a few years following hernia repair (median time between index operation and remedial surgery: 35 and 45 months after open and laparoscopic 
hernia repair, respectively; chapter 4 and chapter 5) supports this hypothesis. Of note, not only the natural evolution of mesh-related pain, but also insufficient knowledge of hernia surgeons and hesitance to refer patients to specialized centres, contribute to this long delay.

Mesh-related pain may occur both after open and endoscopic inguinal hernia repairs. In Western countries, endoscopic repairs such as TEP and TAPP are primarily recommended, providing resources and expertise are available ${ }^{6}$. Endoscopic repairs have proved to decrease complication rates and are cost-effective ${ }^{6}$. In addition, CPIP incidence following an endoscopic inguinal hernia mesh repair is lower (6 to $12.4 \%)^{22,23}$ when compared to an open herniorrhaphy (11 to $16.8 \%)^{4,5,23,24}$. However, it is unknown whether characteristics of chronic pain are different following an endoscopic or an open technique. The number of patients that presents with mesh-related pain is many times higher after open mesh-based repair as compared to endoscopic repairs (chapter 5). An explanation for this difference is possibly provided by the position of the mesh in the preperitoneal space. This location is likely beneficial not only in terms of evoking fewer cases of neuropathic pain ${ }^{22,25}$, but probably also for generating less mesh-related pain. Interestingly, no meshomas were found during laparoscopic mesh removals. Moreover, the majority of meshes was found to be positioned in a completely flat plane (chapter 5). A lower tendency of meshoma formation in the preperitoneal space is possibly related to a relative limited grade of flexing and extending forces when compared to the groin region.

\section{CPIP following endoscopic hernia repair}

Apart from lower rates of mesh-related pain, inguinal nerves are also less frequently affected after endoscopic hernia repair. Lower abdominal and groin anatomy once more provide an explanation for this difference in CPIP rates. The genital branch of the genitofemoral nerve is particularly at risk during an endoscopic minimally invasive procedure as it runs on the bottom of the operative field. In contrast, iliohypogastric and ilioinguinal nerves do not directly cross this surgical plane and are less frequently affected. Profiles of both neuropathic and nociceptive CPIP following endoscopic hernia repair are different compared to CPIP after standard open Lichtenstein repair. Hence, patients with CPIP following endoscopic repair belong to a distinctive subgroup requiring a different diagnostic and therapeutic approach ${ }^{26}$. A tailored work-up proposed by experts is mandatory. 


\section{Central sensitization}

Long lasting inguinal pain may result in central sensitization that can be extremely difficult to distinguish from other potential sources of pain. Pain by central sensitization is independent of any peripheral drive ${ }^{27}$ such as initiated by inguinal nerves and thus is refractory to peripherally directed treatments. Patients with central pain should be excluded from remedial surgery ${ }^{28,29}$. Concise patient history taking and physical examination are helpful for detecting the clinical signs and symptoms associated with centralized pain. For instance, patterns of complaints that cannot be explained by regular neuro-anatomy or the presence of a rare phenomenon of referred visceral pain (Case II) may point towards central sensitization. Differentiating between peripheral neuropathic and centralized pain is difficult as both are associated with sensory skin abnormalities. The response to a peripherally administered diagnostic injection using a local anaesthetic is possibly helpful in distinguishing from central pain and should be included in the standard work-up of CPIP patients.

\section{Other postherniorrhaphy pain syndromes}

It must be appreciated that success rates following remedial surgery are still far from optimal. Apart from nerve- or mesh-related pain, other (and possibly latent) causes of groin pain after the hernia surgery must be considered before institution of remedial surgery, or if remedial surgery is not successful. Three other pain syndromes are frequently encountered, either due to the index hernia surgery, or persisting after the remedial surgery for CPIP including neurectomy and mesh removal. Firstly, recurrent hernias should always be excluded. Secondly, periostitis of the pubic tubercle due to a longstanding direct mechanical irritation or mesh-induced inflammation may persist for months after mesh removal ${ }^{30}$. Remedial surgery itself may also cause a painful local inflammatory reaction but this phenomenon is assumed to be self-limiting. Finally, a long delay between onset of CPIP and remedial therapy may have resulted in an antalgic gait leading to a tendinopathy of the adductor muscles. These pain syndromes have not yet been properly investigated whereas therapeutic options are limited and experimental. It is imperative that, prior to deciding on remedial surgery for CPIP, these patients should be counselled on the potential onset or presence of these pain syndromes. Referred pain originating from the back is an important pitfall when classifying CPIP requiring a thorough physical examination and possibly imaging. In some patients unresponsive to 
all conservative and surgical treatments, deafferentation pain should be considered. This type of pain results from a (complete or partial) disruption of the afferent nerves. As a neurectomy interrupts the normal impulses from the afferent nerves, deafferentation pain is always looming. Why some patients develop deafferentation pain while others do not is still unclear. It is probably worthwhile to evaluate a patient who is unresponsive to remedial surgery in a multidisciplinary team including a pain specialist and a psychologist.

\section{Level of evidence}

Solid, evidence-based preventive and treatment strategies for CPIP are scarce. This paucity of data explains why a consensus regarding treatment options and stratagems for CPIP is not attained ${ }^{13}$. The universally accepted 'level-of-evidence pyramid' indicates that retrospective studies are inferior compared to RCTs. However, retrospective studies are ideal for generating hypotheses and for investigating feasibility and safety of therapies. Moreover, it would be unethical to randomize patients for an invasive treatment before a retrospective evaluation has been performed. Nonetheless, more randomized trials should be conducted to determine true effect sizes of novel therapies.

\section{Nature of the erroneous recall}

In chapter 9 it was demonstrated that recall of pain scores leads to erroneous measurements, especially in patients who still experience pain following therapy. What is the nature of this recalling process? An example that clarifies this finding is recall of women's pain during labour. Postnatal recall of pain intensity results in lower pain scores ${ }^{31}$. These findings can be explained by the theory that a current mood state influences the process of recalling pain ${ }^{32-38}$. Thus, patients who do not experience pain (anymore) at the moment of response, for example women who just gave birth, carrying their newborn in their arms and experiencing a feeling of happiness, underrate previously experienced pain. In other words, patients become accustomed to improvements in their condition, also referred to as 'satisfaction treadmill' 39 , 40. The same theory possibly accounts for patients after a failed surgical treatment. Their negative emotions will modulate memory processing and consequently, probably also reports of pain in the past ${ }^{36}$. In addition, one study also suggests that patients may dramatize pain to receive attention, sympathy or further treatment ${ }^{32}$. This statement is refuted by others who claim that recall bias is not a function of intentional deceit but more an inherent action of the process a brain 
stores and recalls information ${ }^{40-42}$. The concept that recall of pain severity is constructed by a combination of pain intensity and interference with daily activities may add another clarification of the previous findings $s^{43}, 44$. In conclusion, recall pain intensities are influenced by emotions and interference of daily levels of pain at the time of recall, leading to higher and lower pre-intervention pain scores as recalled and reported in failure and in successes, respectively. 


\section{REFERENCES}

1. Usher FC, Cogan JE, Lowry TI. A new technique for the repair of inguinal and incisional hernias. Arch Surg 1960;81:847-54.

2. Chastan P. Tension-free open hernia repair using an innovative self-gripping semi-resorbable mesh. Hernia 2009;13(2):137-42.

3. Franneby U, Sandblom G, Nordin P, et al. Risk factors for long-term pain after hernia surgery. Ann Surg 2006;244(2):212-9.

4. Nienhuijs S, Staal E, Strobbe L, et al. Chronic pain after mesh repair of inguinal hernia: a systematic review. Am J Surg 2007;194(3):394-400.

5. Simons MP, Aufenacker T, Bay-Nielsen M, et al. European Hernia Society guidelines on the treatment of inguinal hernia in adult patients. Hernia 2009;13(4):343-403.

6. Simons MP, Smietanski M, Bonjer HJ, et al. World guidelines for groin hernia management. 2016.

7. Amid PK. Lichtenstein tension-free hernioplasty: its inception, evolution, and principles. Hernia 2004;8(1):1-7.

8. Hassan E. Recall Bias can be a Threat to Retrospective and Prospective Research Designs. The Internet Journal of Epidemiology 2005;3(2).

9. Last JM. A Dictionary of Epidemiology. $4^{\text {th }}$ edition ed. Oxford: Oxford University Press, 2000.

10. Lippman A, Mackenzie SG. What is "recall bias" and does it exist? Prog Clin Biol Res 1985;163C:205-9.

11. Sedgwick P. What is recall bias? BMJ 2012;344.

12. Classification of chronic pain. Descriptions of chronic pain syndromes and definitions of pain terms. Prepared by the International Association for the Study of Pain, Subcommittee on Taxonomy. Pain Suppl 1986;3:S1-226.

13. Kehlet H, Roumen RM, Reinpold W, et al. Invited commentary: Persistent pain after inguinal hernia repair: what do we know and what do we need to know? Hernia 2013;17(3):293-7.

14. Campanelli G, Bertocchi V, Cavalli M, et al. Surgical treatment of chronic pain after inguinal hernia repair. Hernia 2013;17(3):347-53.

15. Grant AM, Scott NW, O’Dwyer PJ. Five-year follow-up of a randomized trial to assess pain and numbness after laparoscopic or open repair of groin hernia. Br J Surg 2004;91(12):1570-4.

16. Aasvang EK, Bay-Nielsen M, Kehlet H. Pain and functional impairment 6 years after inguinal herniorrhaphy. Hernia 2006;10(4):316-21. 
17. Tabbara M, Genser L, Bossi M, et al. Inguinal Hernia Repair Using Self-adhering Sutureless Mesh: Adhesix: A 3-Year Follow-up with Low Chronic Pain and Recurrence Rate. Am Surg 2016;82(2):112-6.

18. Grant AM, Scott NW, O’Dwyer PJ. Five-year follow-up of a randomized trial to assess pain and numbness after laparoscopic or open repair of groin hernia. Br J Surg 2004;91(12):157074.

19. Jennings JM, Ng P. Laparoscopic appendicectomy for suspected mesh-induced appendicitis after laparoscopic transabdominal preperitoneal polypropylene mesh inguinal herniorraphy. J Minim Access Surg 2010;6(1):19-21.

20. Burgmans JP, Voorbrood CE, Simmermacher RK, et al. Long-term Results of a Randomized Double-blinded Prospective Trial of a Lightweight (Ultrapro) Versus a Heavyweight Mesh (Prolene) in Laparoscopic Total Extraperitoneal Inguinal Hernia Repair (TULP-trial). Ann Surg 2016;263(5):862-6.

21. Klosterhalfen B, Klinge U. Retrieval study at 623 human mesh explants made of polypropylene - impact of mesh class and indication for mesh removal on tissue reaction. J Biomed Mater Res B Appl Biomater 2013.

22. Aasvang E, Kehlet H. Chronic postoperative pain: the case of inguinal herniorrhaphy. Br J Anaesth 2005;95(1):69-76.

23. Koning GG, Wetterslev J, van Laarhoven CJ, et al. The totally extraperitoneal method versus Lichtenstein's technique for inguinal hernia repair: a systematic review with meta-analyses and trial sequential analyses of randomized clinical trials. PloS one 2013;8(1):e52599.

24. Perkins FM, Kehlet H. Chronic pain as an outcome of surgery. A review of predictive factors. Anesthesiology 2000;93(4):1123-33.

25. Hallen M, Bergenfelz A, Westerdahl J. Laparoscopic extraperitoneal inguinal hernia repair versus open mesh repair: long-term follow-up of a randomized controlled trial. Surgery 2008;143(3):313-7.

26. Linderoth G, Kehlet H, Aasvang EK, et al. Neurophysiological characterization of persistent pain after laparoscopic inguinal hernia repair. Hernia 2011;15(5):521-9.

27. Devor M. Centralization, central sensitization and neuropathic pain. Focus on "sciatic chronic constriction injury produces cell-type-specific changes in the electrophysiological properties of rat substantia gelatinosa neurons". J Neurophysiol 2006;96(2):522-3.

28. Loos MJ, Scheltinga MR, Roumen RM. Tailored neurectomy for treatment of postherniorrhaphy inguinal neuralgia. Surgery 2010;147(2):275-81. 
29. Kehlet H, Jensen TS, Woolf CJ. Persistent postsurgical pain: risk factors and prevention. Lancet 2006;367(9522):1618-25.

30. Loos MJ, Roumen RM, Scheltinga MR. Classifying post-herniorrhaphy pain syndromes following elective inguinal hernia repair. World J Surg 2007;31(9):1760-5; discussion 66-7.

31. Norvell KT, Gaston-Johansson F, Fridh G. Remembrance of labor pain: how valid are retrospective pain measurements? Pain 1987;31(1):77-86.

32. Salovey P, Smith AF, Turk DC, et al. The accuracy of memory for pain: Not so bad most of the time. APS Journal 1993;2(3):184-91.

33. Bower GH. Mood and memory. Am Psychol 1981;36(2):129-48.

34. Redelmeier DA, Kahneman D. Patients' memories of painful medical treatments: real-time and retrospective evaluations of two minimally invasive procedures. Pain 1996;66(1):3-8.

35. Eich E, Reeves JL, Jaeger B, et al. Memory for pain: relation between past and present pain intensity. Pain 1985;23(4):375-80.

36. Gedney JJ, Logan H. Pain related recall predicts future pain report. Pain 2006;121(1-2):69-76.

37. Williams DA, Park KM, Ambrose KR, et al. Assessor status influences pain recall. J Pain 2007;8(4):343-8.

38. Eich E. On the accuracy of memory for pain. APS Journal 1993;2(3):192-94.

39. Kahneman D. Objective happiness. In: Kahneman D, Deiner, E., Schwarz, N., ed. Well-being: the Foundations of Hedonic Psychology. New York City (NY), USA: Russell Sage Foundation, 1999:3-25.

40. Gendreau M, Hufford MR, Stone AA. Measuring clinical pain in chronic widespread pain: selected methodological issues. Best Pract Res Clin Rheumatol 2003;17(4):575-92.

41. Stone AA, Turkkan JS, Bachrach CA, et al. Belief and feeling: evidence for an accessibility model of emotional self-report. Psychol Bull 2002;128(6):934-60.

42. Stone AA, Turkkan, J. S., Bachrach, C. A., Jobe, J. B., Kurtzman, H. S. The Science of SelfReport: Implications for Research and Practice. Mahwah (NJ), USA: Lawrence Erlbaum Associates, 2000.

43. Broderick JE, Stone AA, Calvanese P, et al. Recalled pain ratings: a complex and poorly defined task. J Pain 2006;7(2):142-9.

44. Von Korff M, Jensen MP, Karoly P. Assessing global pain severity by self-report in clinical and health services research. Spine 2000;25(24):3140-51. 

CHAPTER 11

Future perspectives 


\section{CONSERVATIVE THERAPIES}

Patients with chronic postoperative inguinal pain (CPIP) due to inguinal nerve entrapment presenting to a general practitioner are frequently offered medication including conventional pharmacotherapies such as paracetamol or non-steroidal antiinflammatory drugs (NSAID) $)^{1,2}$. However, effectiveness of these conventional analgesic agents for neuropathic pain syndromes is limited ${ }^{3}$. If nerve-related pain characteristics are recognized, specific neuropathic medications such as gabapentinoids (gabapentin, Neurontin ${ }^{\circledast}$ or pregabalin, Lyrica ${ }^{\circledR}$ ) are considered, since these pharmacological agents may be effective for neuropathic pain ${ }^{4}$. As pharmacotherapeutic neuropathic pain studies particularly focused on diabetic neuropathy ${ }^{5}$, post-herpetic neuralgia ${ }^{6}$ and chemotherapyinduced neuropathic pain ${ }^{7}$, it is highly questionable whether this recommendation can be extrapolated to other neuropathic pain syndromes such as nerve groin entrapment following hernia repair. A literature search identified one case report as the sole evidence for neuropathic medications for inguinal pain following inguinal hernia repair ${ }^{8}$. On the other hand, gabapentinoids use is associated with a large number of serious adverse effects including somnolence and dizziness which may occur in up to $10 \%$ of the patients ${ }^{9}$. Furthermore, ataxia, cervical spasms, disorientation, lethargy and vertigo are often experienced and considered intolerable by patients. Due to the paucity of evidence and these invalidating, psychogenic adverse effects, proper monitoring of the effects of these medications in CPIP patients is required.

Botulinum toxin A (Botox ${ }^{\oplus}$ ) has gained popularity in the realm of facial aesthetics. However, its efficacy was also demonstrated in various types of spasticity and anal fissures. Two trials were published assessing the use of Botox ${ }^{\otimes}$ for peripheral neuropathic pain $^{10,11}$. The substance provides a long-lasting sensory fibre nerve block that lasts longer than local anaesthetics combined with corticosteroids. However, this treatment received a weak recommendation for the indication of peripheral neuropathic pain ${ }^{4}$. Nonetheless, promising results for its use in orchialgia were published ${ }^{12}$. Whether this 'wonder-drug' may also prove efficacious in the treatment of (neuropathic) postsurgical inguinal pain remains to be investigated. A trial comparing investigating botulinum toxin A injections in patients with genitofemoral neuralgia is suggested.

Another minimally invasive treatment option for CPIP could be pulsed radiofrequency (PRF). This technique uses radiofrequency pulses to generate thermal lesions of portions 
of the nerves. Previous studies found that PRF reduced chronic pain in different syndromes ${ }^{13,14}$. A narrative review on the evidence of PRF in CPIP concluded that the evidence was fairly limited ${ }^{15}$. Other studies demonstrated promising results for PRF ${ }^{16,17}$. A future RCT may properly evaluate the effect of PRF on CPIP.

\section{SURGICAL THERAPIES}

The present thesis pays special attention to type of anaesthetic technique that may in part determine success rates of remedial surgery for CPIP. Nowadays, general anaesthesia is applied in up to $70 \%$ of primary inguinal hernia repairs ${ }^{18}$. According to our findings, spinal anaesthesia for remedial surgery may be superior in terms of optimized long term pain relief (chapter 6), although more robust, prospective studies are required to confirm these preliminary findings (chapter 7). Randomized trials comparing anaesthetic techniques on long-term pain relief in patients who are surgically treated for CPIP were not performed. If the hypothesis is confirmed by the SPINASIA trial (chapter 8), spinal anaesthesia may become the preferred technique for remedial surgery for inguinal pain syndromes including CPIP.

A variety of surgical techniques for hernia repair were developed over the last decades. Especially the endoscopic approach for inguinal hernia repair has effectively reduced the prevalence of CPIP. However, absolute numbers of patients suffering from CPIP following TEP or TAPP will nevertheless further rise due to the popularization of endoscopic techniques. Consequently, the need for laparoscopic mesh removal will further increase. Larger series are necessary to identify pros en cons of this novel treatment and to refine the selection process. This highly specialized care should be centralized and must only be performed by trained expert hernia surgeons.

\section{PATHOGENESIS OF POSTHERNIORRHAPHY PAIN}

Postoperative inguinal pain only develops in the minority of patients following (mesh-based) inguinal hernia repair. The question why some do develop chronic pain while other do not is largely unanswered. As previously discussed, nerve entrapment 
and meshoma formation provide two plausible mechanisms. Other possible causes are neuronal myelin degeneration ${ }^{19}$ or formation of a neuroma (a white, oval, firm-elastic palpable nodule) as a consequence of the surgical nerve injury. When a neuroma is identified and removed, success rates of the remedial surgery may be increased (chapter 6). However, a neuroma is just found in $7.7 \%$ of the cases (chapter 7). Earlier research has not unveiled its aetiology ${ }^{20}$. In theory, inflammation evoked by the inserted mesh (chapter 10) may lead to a reactive proliferation of perineural cells in proximity to the mesh $^{20,21}$. More research on the pathogenesis and how to avoid neuroma formation is desirable.

Mesh-induced inflammation may stimulate adjacent nerves to sprout and regenerate, a process called neogenesis. Research on this phenomenon is scarce including an animal study $^{19}$. A recent study shed some light on neogenesis of nerves in removed meshes after previous inguinal hernia repair in CPIP patients ${ }^{22}$. Histopathological examination of removed meshes is not standardly performed but could identify mechanisms for CPIP. All removed meshes, either removed for pain or for infection, are prospectively collected in our centre. Histopathological and immunohistochemical staining methods may reveal several aspects of neuroneogenesis. Research is aimed at studying the relation between the presence of neogenesis and clinical patient characteristics and outcome.

\section{OUTCOME MEASUREMENTS}

Previous studies on CPIP mainly focused on pain relief. To this end, a range of different pain scales were used. However, pain relief is not always proportionally related to patient satisfaction. Satisfaction is a complex and multi-factorial measure of outcome. It depends on pain relief but is also determined by the physician's communication and individual factors such as patient expectation and experience and quality of life. Perhaps this patient-related outcome measure is more appropriate to properly assess the assets of a new treatment. Therefore, it is advised to include patient satisfaction as an important outcome parameter in future research on CPIP.

Patients who still feel pain after (surgical) treatment may dramatize previously pain levels (chapter 9). Negative emotions will modulate memory processing and consequently, reporting pain scores ${ }^{23}$. The pain catastrophizing scale (PCS) is an instrument that is 
developed for research on the mechanisms by which catastrophizing impacts on pain experience ${ }^{24}$. Moreover, the hospital anxiety depression scale (HADS) has also been developed to assess states of depression and anxiety ${ }^{25,26}$. By analyzing these scores in relation to outcome, it is possible to analyze the influence of mental status on success. Especially since it is assumed that some patients have a psychological and/or physiological predisposition to the development of chronic pain, as anxiety and depression are known risk factors for CPIP ${ }^{27-29}$. Implementation of these scores in future research to correct for this important confounding factors is suggested. 


\section{REFERENCES}

1. Vo T, Rice AS, Dworkin RH. Non-steroidal anti-inflammatory drugs for neuropathic pain: how do we explain continued widespread use? Pain 2009;143(3):169-71.

2. Hall GC, Morant SV, Carroll D, et al. An observational descriptive study of the epidemiology and treatment of neuropathic pain in a UK general population. BMC Fam Pract 2013;14:28.

3. Moore RA, Derry S, Aldington D, et al. Amitriptyline for neuropathic pain in adults. Cochrane Database Syst Rev 2015(7):CD008242.

4. Finnerup NB, Attal N, Haroutounian S, et al. Pharmacotherapy for neuropathic pain in adults: a systematic review and meta-analysis. Lancet Neurol 2015;14(2):162-73.

5. Bril V, England J, Franklin GM, et al. Evidence-based guideline: Treatment of painful diabetic neuropathy: report of the American Academy of Neurology, the American Association of Neuromuscular and Electrodiagnostic Medicine, and the American Academy of Physical Medicine and Rehabilitation. Neurology 2011;76(20):1758-65.

6. Dubinsky RM, Kabbani H, El-Chami Z, et al. Practice parameter: treatment of postherpetic neuralgia: an evidence-based report of the Quality Standards Subcommittee of the American Academy of Neurology. Neurology 2004;63(6):959-65.

7. Hershman DL, Lacchetti C, Dworkin RH, et al. Prevention and management of chemotherapyinduced peripheral neuropathy in survivors of adult cancers: American Society of Clinical Oncology clinical practice guideline. J Clin Oncol 2014;32(18):1941-67.

8. Benito-Leon J, Picardo A, Garrido A, et al. Gabapentin therapy for genitofemoral and ilioinguinal neuralgia. J Neurol 2001;248(10):907-8.

9. Farmacotherapeutisch Kompas 2015.

10. Yuan RY, Sheu JJ, Yu JM, et al. Botulinum toxin for diabetic neuropathic pain: a randomized double-blind crossover trial. Neurology 2009;72(17):1473-8.

11. Apalla Z, Sotiriou E, Lallas A, et al. Botulinum toxin A in postherpetic neuralgia: a parallel, randomized, double-blind, single-dose, placebo-controlled trial. Clin J Pain 2013;29(10):85764.

12. Khambati A, Lau S, Gordon A, et al. OnabotulinumtoxinA (Botox) nerve blocks provide durable pain relief for men with chronic scrotal pain: a pilot open-label trial. J Sex Med 2014;11(12):3072-7.

13. Van Zundert J, Patijn J, Kessels A, et al. Pulsed radiofrequency adjacent to the cervical dorsal root ganglion in chronic cervical radicular pain: a double blind sham controlled randomized clinical trial. Pain 2007;127(1-2):173-82. 
14. Van Boxem K, de Meij N, Kessels A, et al. Pulsed Radiofrequency for Chronic Intractable Lumbosacral Radicular Pain: A Six-Month Cohort Study. Pain medicine (Malden, Mass) 2015.

15. Werner MU, Bischoff JM, Rathmell JP, et al. Pulsed radiofrequency in the treatment of persistent pain after inguinal herniotomy: a systematic review. Reg Anesth Pain Med 2012;37(3):340-3.

16. Kastler A, Aubry S, Piccand V, et al. Radiofrequency neurolysis versus local nerve infiltration in 42 patients with refractory chronic inguinal Neuralgia. Pain Physician 2012;15(3):237-44.

17. Kastler A, Aubry S, Barbier-Brion B, et al. Radiofrequency neurolysis in the management of inguinal neuralgia: preliminary study. Radiology 2012;262(2):701-7.

18. Kehlet $\mathrm{H}$, White PF. Optimizing anaesthesia for inguinal herniorrhaphy: general, regional, or local anaesthesia? Anesth Analg 2001;93(6):1367-9.

19. Demirer S, Kepenekci I, Evirgen O, et al. The effect of polypropylene mesh on ilioinguinal nerve in open mesh repair of groin hernia. J Surg Res 2006;131(2):175-81.

20. Foltan R, Klima K, Spackova J, et al. Mechanism of traumatic neuroma development. Med Hypotheses 2008;71(4):572-6.

21. Uzzo RG, Lemack GE, Morrissey KP, et al. The effects of mesh bioprosthesis on the spermatic cord structures: a preliminary report in a canine model. J Urol 1999;161(4):1344-9.

22. Bendavid R, Lou W, Grischkan D, et al. A mechanism of mesh-related post-herniorrhaphy neuralgia. Hernia 2016;20(3):357-65.

23. Gedney JJ, Logan H. Pain related recall predicts future pain report. Pain 2006;121(1-2):69-76.

24. Sullivan MJL, Bishop S, Pivik J. The pain catastrophizing scale: development and validation. Psychol Assess 1995;7:432-524.

25. White D, Leach C, Sims R, et al. Validation of the Hospital Anxiety and Depression Scale for use with adolescents. Br J Psychiatry 1999;175:452-4.

26. Spinhoven P, Ormel J, Sloekers PP, et al. A validation study of the Hospital Anxiety and Depression Scale (HADS) in different groups of Dutch subjects. Psychol Med 1997;27(2):36370.

27. Perkins FM, Kehlet $\mathrm{H}$. Chronic pain as an outcome of surgery. A review of predictive factors. Anesthesiology 2000;93(4):1123-33.

28. Aasvang E, Kehlet H. Chronic postoperative pain: the case of inguinal herniorrhaphy. Br J Anaesth 2005;95(1):69-76.

29. Macrae WA. Chronic pain after surgery. Br J Anaesth 2001;87(1):88-98. 

CHAPTER 12

\author{
Valorisation
}




\section{INTRODUCTION}

Inguinal hernia repair is a very frequently performed procedure in general surgery, with approximately 20 million repairs every year worldwide ${ }^{1}$. In the Netherlands, over 28,000 adult patients underwent inguinal hernia repair in 2010, accounting for 17 operations per 10,000 inhabitants $^{2}$. It is estimated that some $2-6 \%$ of the inguinal hernia repairs result in severe groin pain leading to significant impairment in social and daily activities $^{3-7}$. As a consequence, yearly up to 2,000 Dutch patients will be confronted with chronic postoperative inguinal pain (CPIP) in the Netherlands. As chronic inguinal pain can also develop after other surgical therapies including caesarean sections $(28,644$ in the Netherlands in $2010^{2}$ ), this number of new onset inguinal pain patients is probably an underestimation.

There is no consensus on the optimal treatment strategy for CPIP. This uncertainty is also reflected in the contents of the international guidelines on hernia surgery, as robust recommendations are not provided $^{8}$. Prevention and treatment of inguinal pain clearly needs more scientific evidence. Although remedial surgeries should be performed in specialised centres by experienced and well trained surgeons, there is a necessity for more evidence-based practical guidelines in order to evaluate the safety and efficacy of these interventions. In my opinion it is appropriate to explore remedial surgery options in an earlier phase in the treatment algorithm of CPIP, as a longer delay could cause protracted periods of postherniorrhaphy pain. This is even more true as the phenomenon of 'window of opportunity' may apply in these patients. Surely, once central sensitization occurs, peripherally oriented therapies are probably to no avail.

\section{SOCIAL RELEVANCE}

In the present era of evidence-based medicine, more pain research is performed than ever before. Nevertheless, knowledge regarding determinants, pathogenesis, prevention, treatment and prognosis of pain is still incomplete. CPIP is a obstinate entity that is particularly difficult for patients to accept because of its iatrogenic aetiology. This is even more true as a substantial portion of patients was never counselled on the risk of CPIP prior to surgery. They are often not taken seriously enough by their surgeons who 
performed the index hernia surgery, especially when a relative 'simple' surgical procedure as inguinal hernia repair was performed. Unfortunately, the level of general knowledge of (hernia) surgeons regarding CPIP is suboptimal. Moreover, misconceptions proclaimed by the patient's environment (e.g. family, colleagues, employers) largely contribute to the misery and ignorance these CPIP patients feel.

Acceptance of the phenomenon of CPIP would have been much easier if a biologic substrate underlying CPIP was identified. The present thesis has identified several anatomic substrates explaining inguinal pain (chapter 4, 5, 6, 7, case I, II). These examples may help (surgical) specialists in referring a patient to specialized centres for evaluation and possibly remedial treatment once conservative stratagems are to no avail. Studies from this thesis may also guide these other (para)medics confronted with a CPIP patient. Moreover, a multidisciplinary approach including pain specialists and hernia surgeons is required for a proper advice regarding diagnostic and therapeutic approach of a CPIP patients.

\section{SOCIETAL IMPACT}

In the era of the well-informed patient with access to the world wide web, patients are more aware of the phenomenon of mesh-related CPIP. The use of meshes for inguinal hernia repair is currently subject to increasing criticism including massive suing of large pharmaceutical companies. In 2016 and 2017, a Dutch consumers television programme, called 'RADAR', dedicated several episodes to the use of prosthetic meshes in inguinal hernia repair. They criticised the use of meshes and highlighted cases of CPIP patients. In reaction to this negative publicity, the Dutch Surgical Society (Nederlandse Vereniging voor Heelkunde, $\mathrm{NVvH}$ ) has stated a clear and uniform opinion on the use of prosthetic materials in inguinal hernia repair: It is not ideal, but it is the best solution we have at present.

It must be appreciated that the overwhelming majority of hernia patients will never develop CPIP. One could question whether a tension repair (using the body's own material for closing the defect) has any role nowadays instead of a mesh-based technique. Before introduction of the prosthetic mesh, these procedures were considered standard but recurrences were quite common. Furthermore, a tension repair is associated with an 
increased chance on CPIP as compared to mesh-based repairs ${ }^{9}$. Hence these techniques are not recommended in current international guidelines ${ }^{8}$. Is the patients' reluctance to mesh-based repair justified? As claimed by $\mathrm{NVvH}$, unfortunately, there is no better method at this moment in time.

As reported earlier, up to $6 \%$ of the hernia repairs result in significant restrictions during daily activities ${ }^{3-7}$. This number was estimated some years ago and was therefore mainly based on open repairs. With the increasing use of endoscopic hernia repair techniques, it is likely that the actual number will nowadays be lower. Since the present thesis has introduced and investigated feasible, safe and effective surgical treatments for CPIP, patients may be reassured in the knowledge that successful remedial surgery is possible.

\section{ECONOMICAL RELEVANCE}

A diversity of treatment strategies for CPIP will likely lead to savings in health care costs. Chapter 2 and chapter 3 showed that the (more expensive) self-gripping mesh is not superior regarding pain relief or hernia recurrence rates after three years of follow-up as compared to the conventional sutured meshes in open inguinal hernia repair. By using only conventional meshes for primary open repair, money might be spared.

Previous research on the cost-effectiveness of remedial surgery (i.e. neurectomy) found that a successful neurectomy reduced compensational costs up to $€ 29,000$ per patient a year in the Netherlands and was therefore considered cost-effective ${ }^{10}$. That study assumed a 50\% reduction of occupational disability after a successful neurectomy. No other literature is available on the work resumption after meshectomy. It is expected that an open or laparoscopic mesh removal (chapter 4,5) will have similar or even higher cost reductions as success rates varied from 50-100\% in our literature review (chapter 4). Therefore, implementation of the present results may have substantial financial consequences. Furthermore, improved success rates in selected subgroups of patients (chapter 6) combined with the preferential use of spinal anaesthesia (chapter 7, 8) may lead to a more beneficial cost-effectiveness of remedial surgeries for CPIP.

The SPINASIA trial (chapter 8) aims to determine patient-related outcome measures and costs. These analyses not only pertain to direct costs but also involve the costs 
secondary to a treatment, the so-called indirect costs. Loss of productivity and impact on society must also be taken into account when evaluating the overall impact of CPIP.

\section{RELEVANCE IN THE MEDICAL FIELD}

Mesh removal recommendations for physicians who are specialised in hernia treatment were proposed in chapter 4. A clear list of specifics facilitating selection of appropriate patients potentially benefitting from meshectomy will likely influence remedial surgery success rates leading to an increased number of patients referred for mesh removal. In 2014, a consensus-based algorithm for CPIP was published ${ }^{11}$. In chapter 4 this algorithm was discussed and some modifications were suggested, as based on the findings of the present thesis. Whether imaging including Magnetic Resonance Imaging (MRI) aids in determining whether remedial surgery is required, is highly questionable. If a meshoma is suspected, the present algorithm advises consultation of a pain team and only suggests a meshectomy if pain is refractory to conservative management ${ }^{11}$. In chapter 6 and chapter 7 , it was demonstrated that the presence and subsequent removal of a meshoma were significantly associated with a successful outcome. Therefore, it is suggested to operate on a meshoma when identified.

Surgical therapies for CPIP are often considered a final option once all conservative treatments were to no avail. An unnecessary delay in referral and subsequent adequate therapy may lead to a limited chance on recovery. Thus, it is debatable whether remedial surgery deserves a place higher up in the algorithm of CPIP patients. Our research team has participated in the working group for the latest international guidelines for groin hernia management. This thesis definitely contributes to a broader scientific basis for management of CPIP and results may be included in the upcoming update.

Caution is warranted when interpreting the results of studies using recalled pain scores. Recall bias may lead to an underestimation of the treatment effect when success rates exceed $67 \%$ (chapter 9 ). Conversely, effect sizes are overestimated when success rates are below $67 \%$. These findings should help medical professionals to critically evaluate conclusions of studies. Furthermore, it is suggested to use less detailed pain scores in future research, since more comprehensive scales seem to be more susceptible for recall errors (chapter 9). 


\section{REFERENCES}

1. Kingsnorth A, LeBlanc K. Hernias: inguinal and incisional. Lancet 2003;362(9395):1561-71.

2. Landelijke Medische Registratie (LMR) van ziekenhuisopnamen van Dutch Hospital Data (Utrecht) via Statline Centraal Bureau voor de Statistiek (CBS), Den Haag/Heerlen. Consulted 15 February 2017 via http://statline.cbs.nl/StatWeb/ publication/?VW=T\&DM=SLNL\&PA=80386NED\&LA=NL.

3. Callesen T, Bech K, Kehlet H. Prospective study of chronic pain after groin hernia repair. Br J Surg 1999;86(12):1528-31.

4. Poobalan AS, Bruce J, King PM, et al. Chronic pain and quality of life following open inguinal hernia repair. Br J Surg 2001;88(8):1122-6.

5. Bozuk M, Schuster R, Stewart D, et al. Disability and chronic pain after open mesh and laparoscopic inguinal hernia repair. Am Surg 2003;69(10):839-41.

6. Mikkelsen T, Werner MU, Lassen B, et al. Pain and sensory dysfunction 6 to 12 months after inguinal herniotomy. Anesth Analg 2004;99(1):146-51.

7. Aasvang E, Kehlet H. Chronic postoperative pain: the case of inguinal herniorrhaphy. Br J Anaesth 2005;95(1):69-76.

8. Simons MP, Smietanski M, Bonjer HJ, et al. World guidelines for groin hernia management. 2016.

9. Collaboration EUHT. Repair of groin hernia with synthetic mesh: meta-analysis of randomized controlled trials. Ann Surg 2002;235(3):322-32.

10. Loos MJ, Lemmers CHC, Heineman E, et al. Occupational disability due to chronic postherniorrhaphy neuralgia: a plea for tailored neurectomy. Surgical management of chronic inguinal pain syndromes: Maastricht University, 2011:122-35.

11. Lange JF, Kaufmann R, Wijsmuller AR, et al. An international consensus algorithm for management of chronic postoperative inguinal pain. Hernia 2014. 



\section{CHAPTER 13}

Samenvatting

(Dutch Summary) 


\section{DUTCH SUMMARY (SAMENVATTING)}

\section{Preventie van chronische postoperatieve liespijn}

Sinds de introductie van kunststof matten voor liesbreukchirurgie $1958^{1}$ is het aantal recidief inguinale hernia's ${ }^{a}$ drastisch verminderd. Desalniettemin is het onderzoek naar het optimale matje voor liesbreukherstel nog steeds in volle gang. Er wordt namelijk aangenomen dat het gebruik van lichaamsvreemd materiaal geassocieerd is met chronische (dat is langer dan drie maanden) postoperatieve liespijn (Engels: chronic postoperative inguinal pain, CPIP). Hedentendage is CPIP de meest hardnekkige complicatie van liesbreukherstel en daarom binnen de gezondheidszorg een belangrijk probleem bij uitstek gebleken. In de loop der tijd zijn er vele pogingen gedaan om CPIP te reduceren, maar met onvoldoende effect. Een citaat van Desiderius Erasmus (1466-1536) is hier wellicht op zijn plaats: "beter voorkomen dan genezen".

Bij één van de strategieën om CPIP te voorkómen ligt de focus op onderzoek naar alternatieve matjes. Een zelfklevende mat, die zichzelf vasthecht in het omliggende weefsel met behulp van microhaakjes van vicryl ${ }^{2}$ werd ontwikkeld. Deze mat draagt de toepasselijke naam Progrip ${ }^{\mathrm{Ts}}$. Dit semi-resorbeerbare ${ }^{\mathrm{b}}$, zelfklevende en lichtgewicht matje maakt hechtingen om het matje te fixeren overbodig en zou hierom CPIP kunnen voorkómen. Een vergelijkende studie naar postoperatieve pijn na het implanteren van de conventionele, ingehechte, polypropyleen mat versus de zelfklevende Progrip ${ }^{\mathrm{Ts}}$ mat wordt beschreven in hoofdstuk 2. Er werden geen significante verschillen gevonden met betrekking tot pijnintensiteit na de operatie op de langere termijn. Er werd geconcludeerd dat de ProGrip ${ }^{\text {tw }}$ mat mogelijk wel acute postoperatieve pijn zou verminderen maar geen effect heeft op CPIP.

De studie had een follow-up periode van één jaar. Omdat CPIP een chronisch pijnsyndroom betreft, zou een langere follow-up van de patiënten gepast zijn. De driejaars-resultaten van deze dubbel-geblindeerde gerandomiseerde studie (Engels: randomized controlled trial, $\mathrm{RCT}^{\mathrm{t}}$ ) zijn beschreven in hoofdstuk 3. Naast pijn is er ook gekeken naar het aantal recidief herniaties ${ }^{\mathrm{a}}$, naar karakteristieken van neuropathische ${ }^{\mathrm{d}}$ en nociceptieve $e^{\mathrm{e}}$ pijn en naar seksueel functioneren na liesbreukherstel. Drie jaar na implantatie van de zelfklevende Progrip ${ }^{\text {Tm }}$ mat of het ingehechte polypropyleen matje voor open primair liesbreukherstel, werd er een minimale hoeveelheid patiënten gezien met liespijn. Opvallend is de gestoorde sensibiliteit ${ }^{\mathrm{f}}$ van de lieshuid bij een groot aantal 
patiënten. Ook een corpus alienumgevoelg kwam verrassend veel voor in beide groepen. Zowel deze gestoorde sensibiliteit ${ }^{f}$ als het corpus alienumgevoel ${ }^{g}$ leken één jaar na het liesbreukherstel weer toe te nemen. Het aantal recidieven na drie jaar was aanzienlijk hoger in bij patiënten die de Progrip ${ }^{\mathrm{ma}}$ mat hadden gekregen. Helaas voorkomt of vermindert het zelfklevende matje CPIP niet en is daarom niet aan te raden voor primair open liesbreukherstel.

\section{Chirurgische behandelingen voor CPIP}

CPIP blijft een belangrijk gezondheidsprobleem en de ontwikkeling van effectieve behandelmethoden is traag. De meeste therapieën richten zich op beïnvloeding van de drie lieszenuwen (te weten nervus ilioinguinalis, nervus iliohypogastricus en nervus genitofemoralis, figuur 13.1), omdat aangenomen wordt dat CPIP meestal een neuropathische ${ }^{\mathrm{d}}$ oorzaak heeft. Chirurgische behandelingen voor CPIP, zoals een neurectomie ${ }^{\mathrm{h}}$ van één of meerdere van deze lieszenuwen kan worden overwogen. Echter, er is een selecte groep patiënten waarbij een neurectomie ${ }^{\mathrm{h}}$ en conservatieve behandelingen onvoldoende soelaas bieden. Deze groep patiënten zouden mogelijk een nociceptieve $^{c}$ of inflammatoire ${ }^{i}$ pijn hebben ten gevolge van het matje zelf. De typische kenmerken van een dergelijk pijnsyndroom zijn echter nooit vastgelegd. In hoofdstuk 4 worden deze karakteristieken zorgvuldig omschreven en zouden kunnen dienen als een handvat voor liesbreukspecialisten en andere artsen. Een pure matgerelateerde pijn wordt omschreven als een corpus alienumgevoel ${ }^{g}$ waarbij de pijn verergert tijdens het flecteren $^{j}$ van de heup, zoals bij het autorijden. Pijnverlichting treedt op bij liggen of extensie $^{\mathrm{k}}$ van de heupmusculatuur. Palpatie ${ }^{\mathrm{l}}$ van het ligament van Poupart ${ }^{\mathrm{m}}$ is opvallend pijnlijk. Neuropathische ${ }^{\mathrm{d}}$ kenmerken zoals een hyperpathische ${ }^{\mathrm{n}}$, hypoesthetische ${ }^{\mathrm{o}}$ of allodynische $^{\mathrm{p}}$ huid en triggerpunten ${ }^{\mathrm{q}}$ zijn veelal afwezig. 


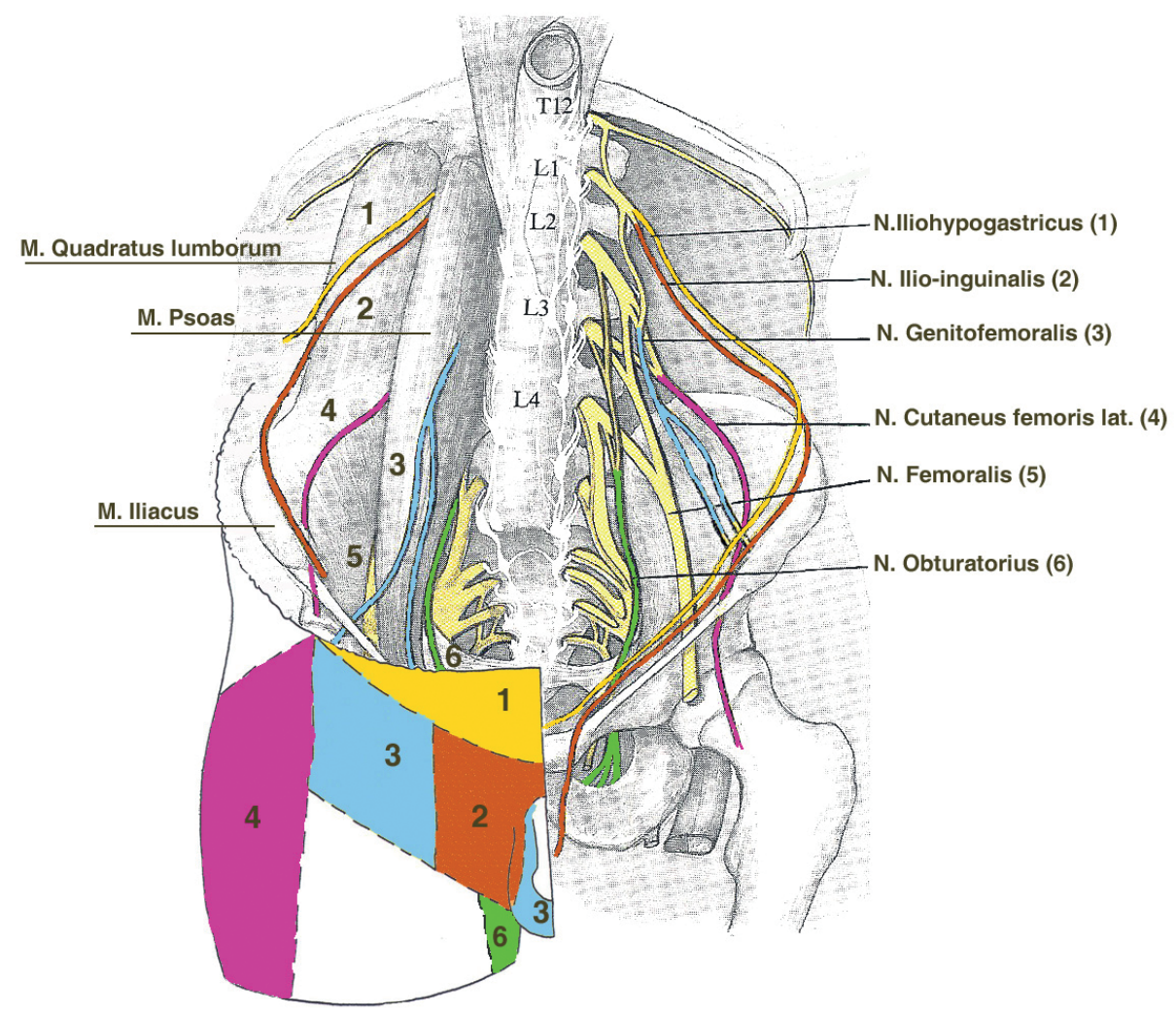

FIGURE 13.1:

Neuro-anatomie van de lieszenuwen

Een retrospectief ${ }^{\mathrm{r}}$ onderzoek bij 74 opeenvolgende patiënten die een open matverwijdering ondergingen voor CPIP na liesbreukherstel volgens de Lichtensteintechniek, al dan niet gecombineerd met een neurectomie ${ }^{\text {h }}$, werd uitgevoerd (hoofdstuk 4, figuur 13.2). Matverwijdering met of zonder een selectieve neurectomie ${ }^{\text {h }}$ bleek succesvol in twee van de drie patiënten met de hierboven benoemde 'matpijn' (mediane ${ }^{u}$ followup periode 18 maanden). Het aantal pijnvrije patiënten na alleen een matverwijdering (63\%) of in combinatie met een neurectomie ${ }^{\mathrm{h}}(64 \%)$ was niet verschillend. In één op de vijftien patiënten bij wie de mat was verwijderd, trad later een recidief liesbreuk op. Er werd aanvullend literatuuronderzoek verricht naar medisch-wetenschappelijke 
onderzoeken naar matverwijderingen (hoofdstuk 3). Gebaseerd op de gunstige resultaten van het literatuuronderzoek en dit retrospectieve $\mathrm{e}^{\mathrm{r}}$ onderzoek is geconcludeerd dat matverwijdering veilig is met een acceptabel complicatiepercentage. Er wordt geadviseerd om een mat te verwijderen, al dan niet in combinatie met een neurectomie ${ }^{\mathrm{h}}$ in selectieve patiënten die onvoldoende baat hebben bij andere behandelingen.

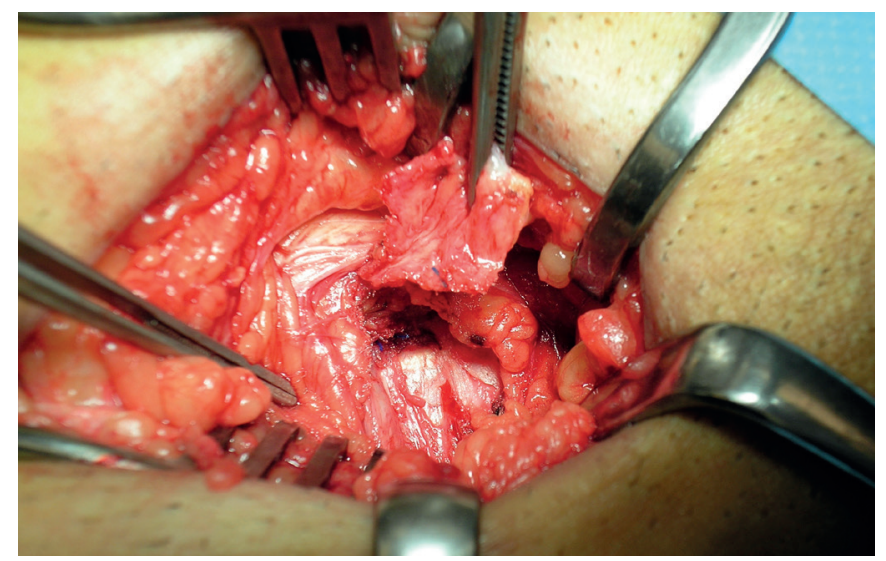

FIGURE 13.2:

Matverwijdering middels een open benadering.

Endoscopisch ${ }^{\mathrm{s}}$ liesbreukherstel (de zogenoemde total extraperitoneal procedure, TEP en transabdominal preperitoneal procedure, TAPP) geeft waarschijnlijk een lagere kans op het ontwikkelen van CPIP ${ }^{3-5}$. Daarnaast zijn deze operatietechnieken kosteneffectief gebleken en is de tijd tot volledig herstel lager ${ }^{6}$. Om deze redenen geeft een groeiend aantal (Westerse) chirurgen de voorkeur aan endoscopisch ${ }^{\mathrm{q}}$ liesbreukherstel boven het conventionele open herstel. Matgerelateerde CPIP na endoscopisch ${ }^{\mathrm{q}}$ liesbreukherstel $^{-}$ komt veel minder voor dan na open herstel. Echter, het aantal endoscopische ${ }^{\mathrm{q}}$ liesbreukoperaties neemt aanzienlijk toe wat leidt tot meer absolute aantallen patiënten met matpijn. Een revolutionaire, nooit eerder in de literatuur gerapporteerde chirurgische techniek waarbij endoscopisch ${ }^{\mathrm{q}}$ geplaatste liesbreukmatten worden verwijderd middels laparoscopie ${ }^{t}$ (figuur 13.3) wordt beschreven in hoofdstuk 5.

De veiligheid en het effect van deze laparoscopische ${ }^{r}$ matverwijdering werd bestudeerd in de eerste 14 patiënten (hoofdstuk 5). De mediane ${ }^{\mathrm{u}}$ operatietijd van deze operatie was 103 minuten en alle matten konden laparoscopisch ${ }^{t}$ worden verwijderd 
zonder dat moest worden geconverteerdv. Er traden, behoudens een peroperatief ${ }^{\mathrm{w}}$ herkend en gecorrigeerde blaasbeschadiging zonder blijvend letsel, geen complicaties op. Mediane ${ }^{\mathrm{u}}$ pijnscores daalden van 8 naar 4 (11-punts Numerieke Rating Schaal, NRS). Patiënttevredenheid was uitstekend tot goed in $71 \%$ van de gevallen. Er werd geconcludeerd dat laparoscopische ${ }^{t}$ matverwijdering uitvoerbaar is voor patiënten met matpijn na endoscopisch ${ }^{\mathrm{s}}$ preperitoneaal $^{\mathrm{x}}$ geplaatste matten.

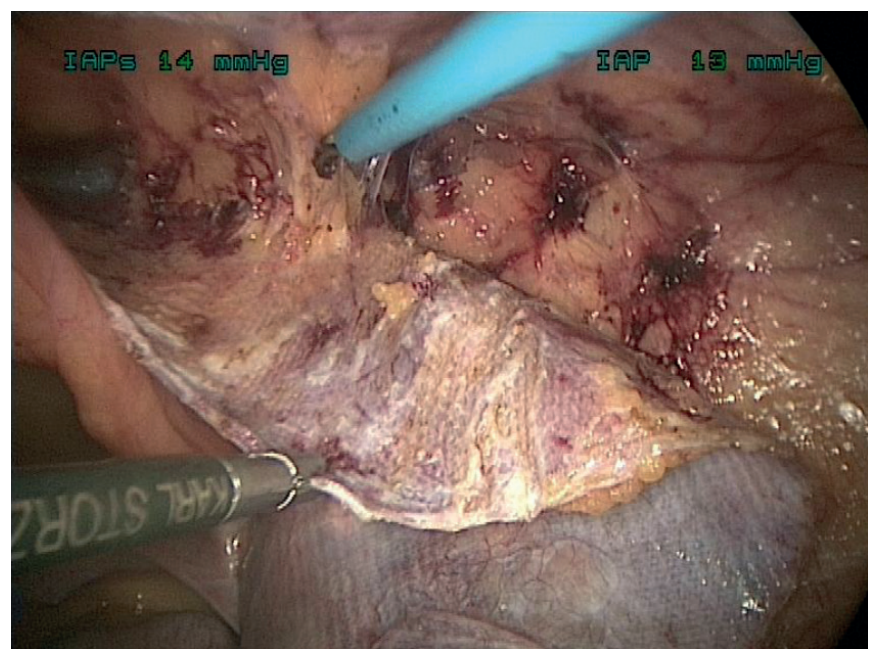

FIGURE 13.3:

Matverwijdering middels laparoscopie (kijkoperatie)

Tijdens liespijnchirurgie zoals een neurectomie ${ }^{\mathrm{h}}$ of een matverwijdering, werden opvallende bevindingen gedaan. De Lichtenstein procedure wordt nog steeds beschouwd als de goudstandaard in ontwikkelingslanden en wordt frequent uitgevoerd in de Westerse wereld $^{6}$. Één van de grote voordelen van deze techniek is de nauwkeurige omschrijving van de te volgen chirurgische stappen ${ }^{7}$. Toch blijven er valkuilen, wat ook blijkt uit een peroperatieve ${ }^{t}$ bevinding waarbij de funiculus spermaticus ${ }^{\mathrm{y}}$ in twee stukken was gedeeld door de geïmplanteerde mat (Casus I, figuur 13.4). Na een selectieve neurectomie ${ }^{\mathrm{h}}$ van de genitale tak van de genitofemorale (figuur 13.1) zenuw werd de patiënt pijnvrij.

Een andere opvallende peroperatieve ${ }^{t}$ bevinding in een tweede patiënt was de ingroei van de appendix vermiformis ${ }^{z}$ na een plug herstel volgens Rutkow en Robbins (Casus 
II, figuur 13.5). De betreffende patiënt had voorafgaand aan de operatie klachten van een corpus alienumgevoel ${ }^{\mathrm{f}}$ in de rechterlies, pijn bij het plassen en een reeds langer bestaande misselijkheid gepaard gaande met een vervelend gevoel in de maagstreek. Na verwijdering van de appendix ${ }^{z}$, die vergroeid bleek met de plug, werd de patiënt pijnvrij en verdwenen zijn klachten van de misselijkheid en vervelend gevoel in de maagstreek. Chirurgen zouden beducht moeten zijn op de mogelijke migratie van liesbreukmatten en een potentieel onderliggende oorzaak van referred ${ }^{\mathrm{aa}}$ viscerale $^{\mathrm{bb}}$ pijn, die kan worden veroorzaakt door de geïmplanteerde mat.

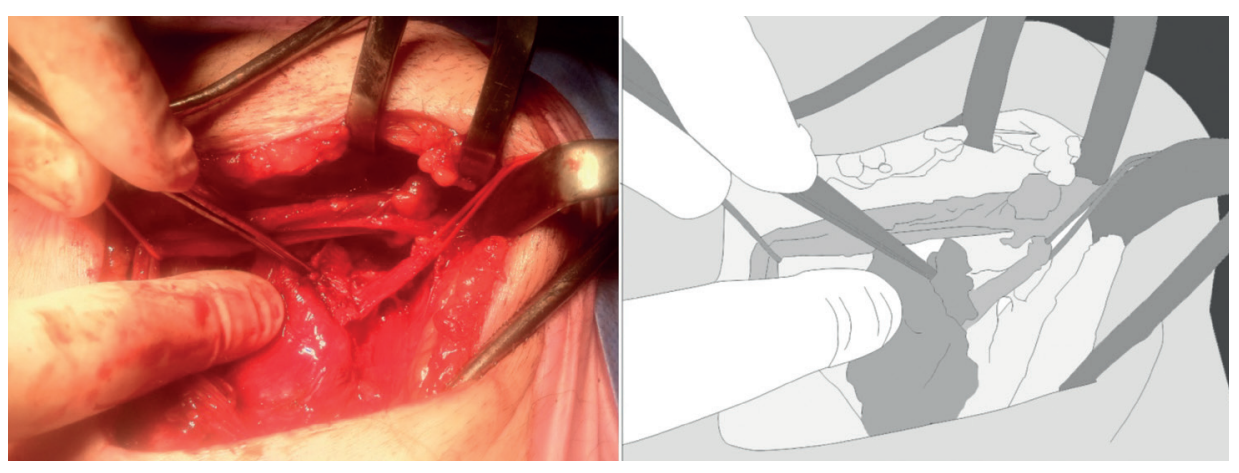

FIGURE 13.4:

De zaadstreng wordt door de eerder geïmplanteerde mat verdeeld in twee stukken

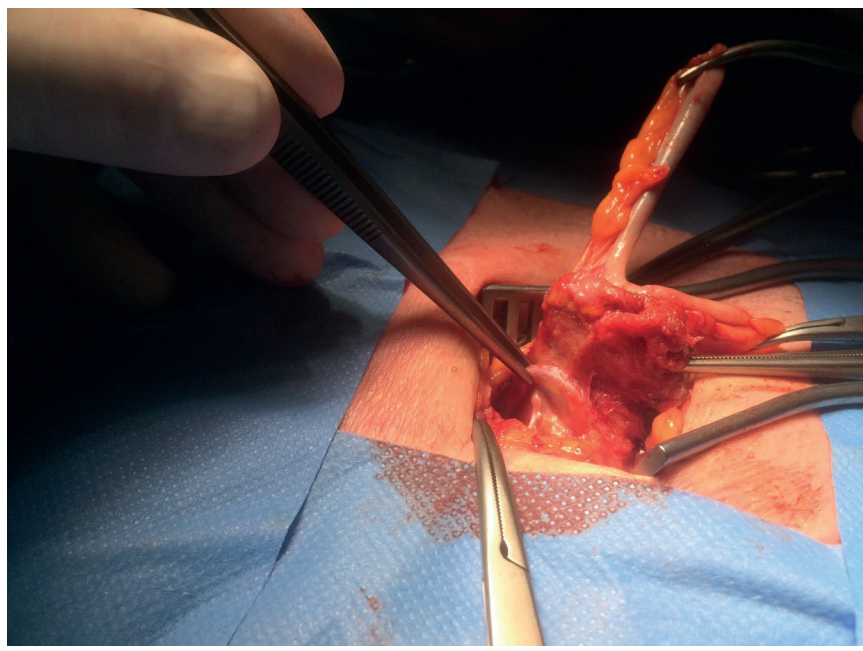

FIGURE 13.5:

De blindedarm is vergroeid met de eerder geïmplanteerde plug 


\section{Optimaliseren van chirurgische behandelingen voor cpip}

De huidige pijnverlichtende operaties voor CPIP zijn veilig en redelijk effectief. Factoren die het succes na liespijnchirurgie beïnvloeden zijn echter tot op heden niet onderzocht. Een retrospectieve ${ }^{r}$ studie werd uitgevoerd om dergelijke potentiële patiënt- en operatiegerelateerde voorspellende factoren te identificeren (hoofdstuk 6). Honderdzesendertig liesoperaties werden geanalyseerd waarbij een multivariabele logistische regressieanalyse suggereerde dat de kans op succes (i.e. pijnverlichting) groter is wanneer de liespijnchirurgie wordt verricht onder spinale anesthesie ${ }^{c c}$, vergeleken met algehele anesthesie $^{\mathrm{dd}}$ (odds ratio ${ }^{\text {ee }} 4,0$ ). Wanneer er tijdens de operatie een meshoom ${ }^{\text {ff }}$ wordt gevonden en verwijderd, worden de kansen op succes ook vergroot (odds ratio ${ }^{\text {ee }}$ $5,3)$. Daarentegen was het preoperatief ${ }^{\mathrm{gg} g}$ gebruik van opioiden ${ }^{\text {hh }}$ geassocieerd met een minder gunstige uitkomst (odds ratio ${ }^{\text {ee }} 0,4$ ). Deze associaties waren opmerkelijk. Om deze reden werd de vraag gesteld of deze resultaten reproduceerbaar waren in een grotere en meer diverse groep patiënten met chronische liespijn. Wanneer spinale anesthesiecc superieur zou blijken, zou dit namelijk een gemakkelijk te beïnvloeden factor zijn om de succeskansen van liespijnchirurgie te vergroten.

Derhalve werd een tweede studie uitgevoerd, gericht op de vraagstelling of spinale anesthesie $^{c c}$ inderdaad de voorkeur heeft boven algehele anesthesie ${ }^{\mathrm{dd}}$ in patiënten die liespijnchirurgie ondergingen voor verschillende oorzaken van de pijn (hoofdstuk 7). Het onderzoek bevatte 339 liespijn patiënten met een diversiteit aan eerdere operaties, variërend van correcties voor liesbreuken en littekenbreuken tot buikwandplastieken en Pfannenstiel incisies ${ }^{\mathrm{ii}}$. De meest uitgevoerde liespijnoperatie was een neurectomie ${ }^{\mathrm{h}}$ $(\mathrm{n}=235)$, waarbij spinale anesthesie $\mathrm{e}^{\mathrm{cc}}$ in $41 \%$ van de patiënten was toegepast. Wederom werd er een positieve associatie gevonden tussen succes en spinale anesthesie ${ }^{c c}$ (odds ratio $^{\text {ee }} 1,9$ ), wat het meest uitgesproken was in de patiëntengroep die een neurectomie ${ }^{\mathrm{h}}$ ondergingen (odds ratio ${ }^{\text {ee }} 2,3$ ). Echter, na een sensitiviteitsanalysej kon worden geconcludeerd dat deze uitkomstmaat in aanzienlijke mate wordt beïnvloed door statistische methoden. Daarom is voorzichtigheid geboden bij interpretatie van deze resultaten.

Om deze reden werd een $\mathrm{RCT}^{\mathrm{k}}$ opgezet om het effect tussen type anesthesie (spinaal ${ }^{c c}$ versus algeheel $^{\mathrm{dd}}$ ) op de pijnverlichting na liespijnchirurgie voor CPIP te onderzoeken, waarbij de bias $^{\mathrm{kk}}$ geassocieerd met retrospectieve ${ }^{\mathrm{r}}$ studies wordt uitgesloten (hoofdstuk 8). Honderdnegentig volwassen patiënten met chronische liespijn zullen worden 
geïncludeerd $^{\text {Il }}$ en gerandomiseerd voor spinale ${ }^{c c}$ of algehele anesthesie ${ }^{\mathrm{dd}}$. Primaire uitkomstmaat is het effect van het type anesthesie op pijnverlichting na een follow-up periode van één jaar. Secundaire uitkomstmaten zijn onder meer patiënttevredenheid, kwaliteit van leven, gebruik van analgetica ${ }^{\mathrm{mm}}$ en (in)directe medische kosten. Potentiële confounders $^{\text {nn }}$ (e.g. peroperatieve ${ }^{\mathrm{w}}$ toediening van andere medicamenten, communicatie, chirurgische stress) worden niet gecontroleerd in deze RCT ${ }^{\mathrm{t}}$. Derhalve is een mogelijk voordelig effect niet aan één specifieke factor te relateren. De toekomstige resultaten van deze $\mathrm{RCT}^{\mathrm{k}}$ moeten dus gezien worden als een effect van de totale setting van het type anesthesie en de geassocieerde factoren. De intentie van het opzetten van de RCT is om klinisch relevante conclusies te trekken die direct kunnen worden toegepast in de

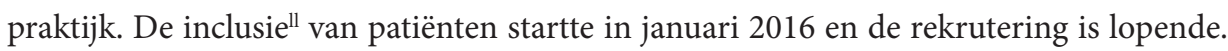
De verwachtte einddatum van de trial is december 2019.

\section{Recall bias}

De meeste studies naar pijn hebben als uitkomstmaat door de patiënt zelf gerapporteerde pijnscores. Recall bias ${ }^{\circ o}$ (een subtype van informatiebias ${ }^{\mathrm{pP}}$ ) komt vaak voor in retrospectieve ${ }^{\mathrm{r}}$ studies, maar kan ook vóórkomen in prospectieve ${ }^{\mathrm{qq}}$ cohort $^{\mathrm{rr}}$ studies, en zelfs in $\mathrm{RCTs}^{\mathrm{c}}$. Dit type bias ${ }^{\mathrm{kk}}$ is gebaseerd op het feit dat patiënten informatie vaak inaccuraat of incompleet herroepen. Wanneer dit onevenredig verdeeld is tussen groepen, zorgt dit mogelijk voor beïnvloeding van de interne validiteit ${ }^{\mathrm{ss}}$ van een studie $^{8-11}$. Omdat veel klinisch onderzoek zich baseert op deze zelfgerapporteerde pijnscores, is betrouwbaarheid hiervan cruciaal. In hoofdstuk 9 wordt het onderzoek dat de mate van invloed van recall bias ${ }^{\circ o}$ op de uitkomsten van chirurgische studies met pijn als uitkomstmaat bestudeert, beschreven.

Uit de studie bleek dat het retrospectief ${ }^{\mathrm{r}}$ verzamelen van pijnscores in studies die de effectiviteit van chirurgie voor chronische buikwand- en liespijn onderzoeken, leidt tot structurele meetfouten van de pijnintensiteit. Door deze meetfout ontstaat er misclassificatie van patiënten die dus onterecht als succes (pijnvrij) worden geclassificeerd, dan wel onterecht als niet-succesvol (ervaart nog pijn) worden geclassificeerd. Deze misclassificatie blijkt in $13,7 \%$ van de patiënten op te treden. Positieve recall misclassificatie (niet-succesvol wordt foutief gemeten als succes) komt vaker voor dan negatieve recall misclassificatie (succes wordt foutief gemeten als niet-succesvol), met een overall gepoolde $^{\mathrm{tt}}$ odds ratio ${ }^{\mathrm{ee}}$ van 2,4. Patiënten met een niet-succesvolle uitkomst, en 


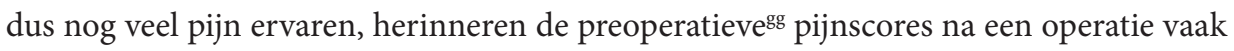
hoger dan deze in werkelijkheid waren (op basis van daadwerkelijk preoperatief ${ }^{\mathrm{gg}}$ gemeten pijnscore). Omgekeerd schatten succesvol geopereerde (en dus pijnvrije) patiënten hun pijnscores lager in wanneer deze worden herroepen. Kortom, het gebruik van herroepen pijnscores heeft een significante effect op het meten van chirurgische uitkomsten in patiënten die lijden aan buikwand- of liespijn. Daarbij is dit effect afhankelijk van de succeskans van een operatie.

\section{CONCLUSIES}

1. Zelfklevende matjes voor open liesbreukherstel zijn niet superieur vergeleken met conventionele, ingehechte matten met betrekking tot CPIP, maar zijn daarentegen wel geassocieerd met een hogere recidiefkans op herniaties ${ }^{\mathrm{a}}$ na drie jaar.

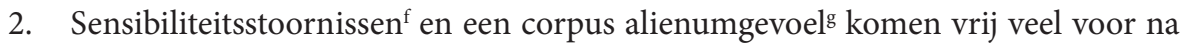
open liesbreukherstel en nemen toe met de tijd.

3. Matgerelateerde liespijn is gekarakteriseerd door een corpus alienumgevoel ${ }^{\mathrm{g}}$, verergert door flexie ${ }^{j}$ van de heup (e.g. autorijden), verlicht in liggende positie en kan worden uitgelokt door palpatie ${ }^{1}$ over het ligament van Poupart $^{\mathrm{m}}$, terwijl neuropathische ${ }^{\mathrm{d}}$ karakteristieken afwezig zijn.

4. Open matverwijdering is effectief in twee op de drie patiënten met matgerelateerde CPIP na open liesbreukherstel.

5. Laparoscopische $e^{s}$ matverwijdering is een veilige, technisch goed uitvoerbare en mogelijk effectieve therapie in de meeste patiënten met matgerelateerde CPIP na endoscopische $e^{s}$ matplaatsing.

6. De kans op pijnvermindering na liespijnchirurgie is mogelijk groter wanneer deze

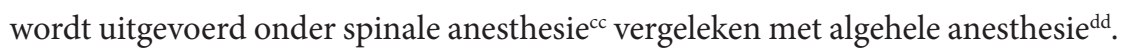

7. Wanneer een meshoom ${ }^{\mathrm{ff}}$ peroperatief ${ }^{\mathrm{w}}$ wordt geïdentificeerd, wordt verwijdering hiervan geadviseerd omdat de kans op reductie van pijn toeneemt en de mogelijke invasie van de mat in naburige viscera ${ }^{\mathrm{bb}}$ wordt vóórkomen.

8. Herroepen pijnscores hebben een significant effect op de uitkomst van retrospectieve ${ }^{\mathrm{r}}$ liespijnstudies. De mate van dit effect wordt bepaald door de succeskans van de interventie. 


\section{LEGENDA VOETNOTEN}

${ }^{\mathrm{a}}$ hernia = breuk; inguinale hernia $=$ liesbreuk

${ }^{\mathrm{b}}$ semi-resorbeerbaar $=$ deels oplosbare

${ }^{\mathrm{c}} \mathrm{RCT}=$ gerandomiseerde studie, onderzoek waarbij patiënten op basis van toeval worden ingedeeld voor een behandeling

${ }^{\mathrm{d}}$ neuropathisch $=$ zenuw-gerelateerd

${ }^{\mathrm{e}}$ nociceptief $=$ niet-zenuw- of aan weefselschade-gerelateerd

${ }^{\mathrm{f}}$ sensibiliteit = gevoelszin; gestoorde sensibiliteit = gevoelsstoornissen

${ }^{\mathrm{g}}$ corpus alienumgevoel $=$ vreemdlichaamgevoel

${ }^{\mathrm{h}}$ neurectomie $=$ het doorsnijden van een zenuw

${ }^{\mathrm{i}}$ inflammatoir $=$ ontstekingsgerelateerd

j flecteren, flexie $=$ buigen in een gewricht waardoor de hoek tussen de botten vermindert

${ }^{\mathrm{k}}$ extenderen, extensie $=$ strekken in een gewricht waardoor de hoek tussen de botten vergroot

${ }^{1}$ palpatie $=$ uitwendig (of inwendig) voelen aan een patiënt als onderdeel van het geneeskundig onderzoek

${ }^{\mathrm{m}}$ ligament van Poupart, ligamentum inguinale $=$ bindweefselstructuur tussen het schaambot en het bekken

${ }^{\mathrm{n}}$ hyperpathie $=$ toegenomen gevoeligheid voor pijnprikkels

${ }^{\circ}$ hypoesthesie, hypesthesie $=$ verminderde gevoeligheid

${ }^{\mathrm{p}}$ allodynie $=$ niet-pijnlijke prikkels worden als hevig pijnlijk ervaren

$\mathrm{q}$ triggerpunt = klein maximaal pijnlijk gebiedje, bijvoorbeeld in de lies

${ }^{\mathrm{r}}$ retrospectief $=$ in de tijd terugkijkend

s endoscopie, endoscopisch = kijkoperatie(f)

${ }^{\mathrm{t}}$ laparoscopie, laparoscopisch = kijkoperatie(f) in de buikholte

" mediaan (statistiek) $=$ het midden van een geordende gegevensverzameling

$\mathrm{v}$ conversie van laparoscopie $=$ omzetten naar een buikwandsnee, een zogenoemde (laparo)tomie

${ }^{\mathrm{w}}$ peroperatief, intraoperatief $=$ tijdens de operatie

${ }^{\mathrm{x}}$ preperitoneaal $=$ voor het buikvlies

${ }^{\mathrm{y}}$ funiculus spermaticus $=$ zaadstreng 
z appendix vermiformis = wormvormig aanhangsel, beter bekend als (alhoewel anatomisch incorrect) 'de blinde darm'

aa referred $=$ zijn origine elders hebbende

${ }^{\mathrm{bb}}$ viscera $=$ ingewanden, visceraal $=$ met betrekking tot de ingewanden

${ }^{c c}$ spinale anesthesie $=$ ruggenprik

dd algehele anesthesie $=$ narcose

ee odds ratio $=$ verhouding tussen twee wedverhoudingen ('odds'). De wedverhouding is de verhouding tussen de waarschijnlijkheid dat een gebeurtenis zal voorvallen en de waarschijnlijkheid dat ze niet zal voorvallen

${ }^{\mathrm{ff}}$ meshoom = opgevouwen of gekrulde mat met volumewerking

${ }^{\text {gg }}$ preoperatief $=$ voor de operatie

hh opioiden $=$ pijnstillers die behoren tot de groep morfineachtige sterke pijnstillers

ii Pfannenstiel incisie $=$ keizersnede

ji sensitiviteitsanalyse = onderzoek waarbij wordt gekeken in welke mate het resultaat wordt beïnvloed door verandering van methode, waarden, variabelen of uitgangspunten $\mathrm{kk}$ bias $=$ vertekening in resultaten

${ }^{11}$ includeren $=$ opnemen in een onderzoek

$\mathrm{mm}$ analgetica $=$ pijnstilling

${ }^{\mathrm{nn}}$ confounder $=$ een variabele, geassocieerd met zowel de te onderzoek variabele als met de uitkomstmaat, waardoor de confounder het verband tussen deze twee verstoort ${ }^{\text {oo }}$ recall bias $=$ vertekening van resultaten door het herroepen van resultaten

$\mathrm{pp}$ informatiebias $=$ vertekening van resultaten door een meetfout

qq prospectief $=$ vooruitkijkend in de tijd

${ }^{\mathrm{rr}}$ cohort studie = een onderzoek waarbij een vooraf geselecteerde groep patiënten gedurende een vastgestelde periode gevolgd worden

ss interne validiteit = mate waarin het redeneren binnen een onderzoek correct is

"t poolen $=$ statistisch combineren van effectschattingen 


\section{REFERENTIELIJST}

1. Usher FC, Cogan JE, Lowry TI. A new technique for the repair of inguinal and incisional hernias. Arch Surg 1960;81:847-54.

2. Chastan P. Tension-free open hernia repair using an innovative self-gripping semi-resorbable mesh. Hernia 2009;13(2):137-42.

3. Franneby U, Sandblom G, Nordin P, et al. Risk factors for long-term pain after hernia surgery. Ann Surg 2006;244(2):212-9.

4. Nienhuijs S, Staal E, Strobbe L, et al. Chronic pain after mesh repair of inguinal hernia: a systematic review. Am J Surg 2007;194(3):394-400.

5. Simons MP, Aufenacker T, Bay-Nielsen M, et al. European Hernia Society guidelines on the treatment of inguinal hernia in adult patients. Hernia 2009;13(4):343-403.

6. Simons MP, Smietanski M, Bonjer HJ, et al. World guidelines for groin hernia management. 2016.

7. Amid PK. Lichtenstein tension-free hernioplasty: its inception, evolution, and principles. Hernia 2004;8(1):1-7.

8. Hassan E. Recall Bias can be a Threat to Retrospective and Prospective Research Designs. The Internet Journal of Epidemiology 2005;3(2).

9. Last JM. A Dictionary of Epidemiology. 4th edition ed. Oxford: Oxford University Press, 2000.

10. Lippman A, Mackenzie SG. What is "recall bias" and does it exist? Prog Clin Biol Res 1985;163C:205-9.

11. Sedgwick P. What is recall bias? BMJ 2012;344. 



\section{Dankwoord}

\section{List of publications}

Curriculum vitae auctoris 


\section{DANKWOORD}

Professor doctor van Kleef, beste Maarten, uw kritische blik op de studies en de discussie over methodologie hebben mij op scherp gezet tijdens het promotietraject. $U$ bent recht door zee en windt er geen doekjes om, eigenschappen die ik erg kan waarderen. Uw visie vanuit de pijngeneeskunde op de discussiepunten hebben een extra dimensie gegeven aan het proefschrift. Hartelijk dank dat u mijn promotor wilt zijn en daarnaast veel dank voor uw steun van mijn ambities om epidemiologie te studeren.

Doctor Roumen, beste Rudi, geniale ideeën en een toewijding aan wetenschap en chirurgie waar menig ander specialist nog wat van kan leren. De voortgangsgesprekken zijn iedere keer weer een extra boost geweest om mijn doelen te bereiken. Innovatieve theorieën en controversiële standpunten innemen zijn voor jou geen bijzonderheid. De waarde van gedegen lichamelijk onderzoek, wat in de huidige geneeskunde steeds meer op de achtergrond verdwijnt heb jij als opleider alle door jou opgeleide chirurgen in spe meegegeven.

Doctor Scheltinga, beste Marc, ongekende passie voor wetenschap en een ongeëvenaarde snelheid waarmee jij stukken grondig reviseert zijn werkelijk op de grens van het mogelijke. Regelmatig kreeg ik een mail in mijn postvak waarin jij op een ludieke manier aangaf dat ik vaart moest zetten achter het afronden van een stuk: "ik verveel me.... Daarnaast een onuitputtelijke bron nieuwe vraagstukken voor op te zetten studies en een aanstekelijk enthousiasme. Wie schrijft die blijft en schrijven zul je.

Geachte leden van de beoordelingscommissie, professor doctor Bouvy, professor doctor Stassen, professor doctor Huygen, doctor Nienhuijs en doctor Sommers, hartelijk dank voor de beoordeling van mijn manuscript.

Chirurgen, SEH-artsen, arts-assistenten chirurgie en mijn (inmiddels ex-)collega's op de SEH van MMC, veel dank voor de steun voor zowel mijn wetenschappelijke als klinische ontwikkelingen in de afgelopen jaren. Dit is mede te danken aan de creatieve en bovenal efficiënte oplossingen om klinische ervaring te combineren met het 
promotietraject binnen een beperkt tijdsbestek. Met veel plezier kan ik jullie in december 2018 wederom mijn collega's noemen.

Arts-onderzoekers chirurgie MMC, dank jullie wel voor het delen van de deugden maar ook de frustraties die het promotietraject met zich mee brengt. De 'onderzoeksweekendjes' hebben erg geholpen bij de nodige ontspanning.

Arts-assistenten heelkunde MUMC, dank voor de welkome ontvangst in het gesticht'. Ik kijk uit naar de uitjes buiten het werk om.

Doctorandus Admiraal-van de Pas, beste Yvonne, hartelijk dank voor je begeleiding van mijn loopbaan en daarmee voor je indirecte bijdrage aan dit proefschrift.

Vrienden, de laatste jaren was het veel en hard werken, maar hard werken loont. Omdat het werk soms de overhand had, waardeer de vriendschap en jullie steun des te meer. Er is gelukkig meer in het leven dan werk en daarom veel dank voor de nodige ontspanning, ook voor in de toekomst.

Hanneke Keijers, doctor Dieleman, beste Jeanne, Eugenie Delvaux, drijfveren achter veel van de proefschriften die MMC voortbrengt, waaronder het mijne. Veel dank voor jullie bijdragen!

Doctor ingenieur Papen-Botterhuis, beste Nicole, de gedrevenheid en het enthousiasme waarmee jij de onderzoeksgroep van de heelkunde leidt werkt aanstekelijk. Ik heb veel vertrouwen in een vruchtbare samenwerking in de toekomst. Een citaat van Scheltinga is hier op zijn plaats: "Science races on!"

Co-auteurs, hartelijk dank voor jullie bijdragen aan mijn proefschrift en de studies. In het bijzonder doctor Slooter, doctor Perquin, doctorandus le Mair. Gerrit, Christel en Léon, dank voor de effectieve en prettige samenwerking en jullie interesse en steun in mijn klinische werkzaamheden en opleiding. 
Ouders, schoonouders, broers en zus, schoonbroer en aanhang, onvoorwaardelijke steun en interesse in mijn drukke bezigheden blijven een grote drijfveer voor mij. Dank voor deze steun in de afgelopen jaren en bij voorbaat alvast voor in de toekomst!

Mijn paranimfen, Johan en Erik, de keuze voor paranimfen was niet zo moeilijk. Gelukkig zeiden jullie toe want meer briljante paranimfen zijn er niet te vinden. Dit kan iedereen die met jullie heeft gewerkt bevestigen. Ik ben blij dat jullie op deze dag aan mijn zijde staan om hopelijk één der prachtige stellingen op te lezen. En dat jullie op het feest natuurlijk iedereen de hele avond bier brengen.

Lieve Leonie, zoals het werk van de chirurgijn voort is gekomen vanuit het werk van de barbier, zo is het vooral aan jou te danken dat ik dit proefschrift en de loopbaancarrière tot nu toe heb kunnen realiseren. Jouw passie voor je vak is inspirerend voor mijn eigen carrière. Ik hou van je schat en geniet van onze tijd samen in Uden. Meer dan genoeg redenen om het proefschrift aan jou op te dragen lijkt mij. Als je haar maar goed zit... 


\section{LIST OF PUBLICATIONS}

\section{Publications}

1. Zwaans WA, Nieuwenhuizen L, Mans SJ. 'A rare manifestation of spontaneous renalureteral haemorrhage in a haemophilia A patient'. Submitted.

2. Zwaans WA, Scheltinga MR, Roumen RM. 'Entrapment of the Appendix in a Meshoma following Inguinal Hernia Repair'. Under review.

3. Zwaans WA, Verhagen T, Wouters L, Loos MJ, Scheltinga MR, Roumen RM. 'A randomized controlled trial with the self-gripping Progrip ${ }^{\text {Tm }}$ mesh versus sutured polypropylene mesh for open inguinal hernia repair: Long-term results on pain and recurrence rates.' Ann Surg 2017. doi: 10.1097/SLA.0000000000002331.

4. Slooter GD*, Zwaans $\mathbf{W A}^{\star}$, Perquin CW, Roumen RM, Scheltinga MR. 'Laparoscopic mesh removal for otherwise intractable inguinal pain following endoscopic hernia repair is feasible, safe and may be effective in selected patients.' Surg Endosc 2017. doi: 10.1007/s00464-017-5824-2. * shared first author

5. Zwaans WA, Koning GG, Gurusamy KS,van Kleef M, Scheltinga MR, Roumen RM. 'Surgical Interventions for Treating Chronic Groin Pain after Hernia Repair (Postherniorrhaphy Inguinodynia)'. Cochrane Database Syst Rev 2017; Issue 4. Art. No.: CD012630. doi: 10.1002/14651858.CD012630.

6. Zwaans WA, le Mair LH, Scheltinga MR, Roumen RM. 'Spinal versus General Anaesthesia in Surgery for Inguinodynia: a Randomised Controlled Trial (SPINASIA trial): study design'. Trials 2017; 18:23. doi: 10.1186/s13063-016-1746-x.

7. Leclercq WK, Zwaans WA, Veerman M, Daalderop N, Legemaate J, Scheltinga MR. 'Shared decision making and surgical informed consent in general surgery: a pilot study on differences in perspectives of physicians and patients'. Submitted. 
8. Leclercq WK, Zwaans WA, Veerman M, Stiggelbout AM, Legemaate J, Scheltinga MR. 'The effect of a best practice surgical informed consent form on shared decision making and surgical informed consent processes'. Submitted.

9. Braeken D, Haydary Y, Zwaans WA, Rohde GG. 'Systematic Review of the Microbiome of the Lung in Patients with Chronic Obstructive Pulmonary Disease'. To be submitted.

10. Brands-Appeldoorn A, Maaskant AJ, Zwaans, WA, Dieleman JP, Schenk KE, Roumen RM. 'Patient Outcome Measurement of Cosmetic Results after BreastConserving Therapy compared to Physicians Judgments'. Submitted.

11. Zwaans WA, Perquin CW, Loos MJ, Roumen RM, Scheltinga MR. 'Mesh removal and Selective Neurectomy for Persistent Groin Pain following Lichtenstein Repair'. World J Surg 2017; 41(3):701-712. doi: 10.1007/s00268-016-3780-y.

12. Verhagen T, Zwaans WA, Loos MJ, Charbon JA, Scheltinga MR, Roumen RM. Inguinal pain after open hernia repair: a single center, double blind randomized trial comparing the self-gripping Progrip with a standard polypropylene mesh. Br J Surg 2016; 103(7):812-8. doi: 10.1002/bjs.10178.

13. Zwaans WA, Scheltinga MR, Roumen RM. 'A Remarkable Pitfall in inguinal Open Mesh Herniorrhaphy - A Case Report'. J Gastrointest Dig Syst 2015; 5:5. doi: 10.4172/2161-069X.1000343.

14. Zwaans WA, van Kuijk SM, le Mair LH, van Kleef, M, Scheltinga MR, Roumen RM. 'Chronic Postoperative Groin Pain Requiring Remedial Surgery: Spinal or General Anaesthesia?'. Under Review.

15. Zwaans WA, de Bruijn JA, Dieleman JP, Steyerberg EW, Scheltinga MR, Roumen RM. 'Recall Bias in Pain Scores Evaluating Abdominal Wall and Groin Pain Surgery: a Meta-Analysis. Under review. 
16. Zwaans WA, Verhagen T, Roumen RM, Scheltinga MR. 'Factors Determining Outcome after Surgery for Chronic Groin Pain Following a Lichtenstein Hernia Repair. World J Surg. 2015; 39(11):2652-62. doi: 10.1007/s00268-015-3183-5.

17. Zwaans WA, Mallia P, van Winden ME, Rohde GG. 'The Relevance of Respiratory Viral Infections in the Exacerbations of Chronic Obstructive Pulmonary Disease - a Systematic Review. J Clin Virol.2014 Oct;61(2):181-8. doi: 10.1016/j.jcv.2014.06.025.

\section{Book chapters}

Zwaans WAR, Scheltinga MRM, Roumen RMH. Aetiology, Pathogenesis and Assessment of Chronic Pain after Inguinal Hernia Repair (Chapter 41). In: Campanelli (Ed.), The Art of Hernia - A Step by Step Guide. Springer Nature, Heidelberg, 2017.

\section{Published abstracts}

Sevonius D, Montgomery A, Smedberg S, Sandblom G, Zwaans W, Perquin C, Roumen R, Scheltinga M, Verhagen T, Loos MJ, Scheltinga MR, Roumen RM, Bökkerink W, Van Laarhoven CJ, Koning GG, Yang ZF, Shen WZ, Lu C, Zheng WH, Xiao WF, Li XR, Narita M, Matsusue R, Hata H, Yamaguchi T, Otani T, Ikai I, Karampinis I, Weiss J, Pilz L, Post S, Herrle F, Murphy J, Yang F, Meng LQ, Meng XZ, Chong YZ, Zhao HY, Liu JG, van Assen T, Brouns JA, Scheltinga MR, Roumen RM. Inguinal Hernia: Post OP Chronic Pain. Hernia. 2015 Apr;19 Suppl 1:S99-S103. doi: 10.1007/BF03355334. No abstract available.

Zwaans W, Verhagen T, Roumen R, Scheltinga M, Dieleman J, Vad M, Frost P, Rosenberg J, Svendsen SW, Tabbara M, Carandina S, Bossi M, Polliand C, Barrat C, Soler M, Chander J, Sharma R, Lal R, Pradhan G, Mol F, Mitura K, Romanczuk M, Lundstrom K, Nordin P, Holmberg H, Sahin A, Kulacoglu H, Olcucuoglu E, Guadalaxara A, Rampinelli LA, Mariani P, Ubezio D, Felici A, Santagati G, Novellino L, Gitelis M, DeAsis F, Chao S, Lapin B, Denham W, Linn J, Haggerty S, Carbray J, Ujiki M, Evans L, Canciani GN, Gordon EM, Wei P, Tam K, Chen C, Kao C, Protasov A, Bogdanov D, Meshkov M, Kurganov I, Misra MC, Bansal VK, Subodh K, Krishna A, Bansal D, Ray S, Rajeshwari S, Marzo F, Forni S, Massi M, Gia L, Dabrowski A. Topic: Inguinal Hernia - Post op chronic pain: incidence, evaluation, legal consequences, therapy, follow up. Hernia. 2015 Apr;19 Suppl 1:S267-74. doi: 10.1007/BF03355370. 


\section{Awards}

2017 Best Clinical Research Award ESSR (for RCT with the self-gripping Progrip mesh versus sutured polypropylene mesh for open inguinal hernia repair: Long-term results on pain and recurrence rates)

\section{Oral presentations}

2017 International Meeting of the European Society of

Amsterdam

Surgical Research (ESSR)

2017 Annual congress of the European Hernia Society

Wenen

2017 Chirurgendagen 2017

Veldhoven

2017 Wetenschapsavond Máxima Medisch Centrum

Veldhoven

2016 Symposium Experimenteel Onderzoek Heelkundige Specialismen

Utrecht

2016 Mini-symposium SolviMáx

Eindhoven

2016 International Meeting of the European Society of

Surgical Research (ESSR)

Praag

2016 Chirurgendagen 2016

Veldhoven

2015 Invited speaker at hospital wide rounds on pain issues - North York Toronto General Hospital

2015 New European Surgical Academy Conference

Berlijn

2015 Chirurgendagen 2015

Veldhoven

2015 World Conference on Abdominal Wall Hernia Surgery

Milaan

2015 Wetenschapsavond Máxima Medisch Centrum

Veldhoven

2014 Rotterdam Interactive Congress on Hernia (RICH)

Rotterdam

\& Symposium on European Prospective Registries for

Ventral Abdominal Wall Hernia

2014 MOSA Conference

Maastricht 


\section{Poster presentations}

2016 International Meeting of the European Society of Surgical Research (ESSR)

2016 International Congress European Hernia Society

Rotterdam

2016 Wetenschapsavond Máxima Medisch Centrum

Veldhoven

2015 World Conference on Abdominal Wall Hernia Surgery

Milaan

2015 Wetenschapsavond Máxima Medisch Centrum

Veldhoven

2015 Chirurgendagen 2015

Veldhoven

2010 Clinical Investigator Science Symposium

Maastricht 
List of publications | 255 


\section{CURRICULUM VITAE AUCTORIS}

Willem Antonius Richardus Zwaans was born on February, 25th 1988 in Schaijk (North Brabant), the Netherlands. He graduated from high school in 2006 (Athenaeum, Mondriaan College Oss), and worked thereafter one year full-time as a cook and studied Italian.

In 2007, he started 'Biomedical Sciences' at the University of Amsterdam and finished within three years with a bachelor thesis on a comparison between 'neurally adjusted ventilatory assist (NAVA) versus pressure support ventilation in adult critically ill patients' (Academic Medical Center, Amsterdam). He was subsequently elected to earn a master degree in 'Medicine and Clinical Research' (Arts-

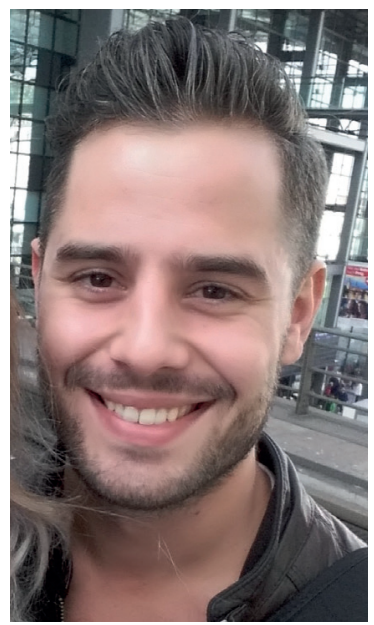
Klinisch Onderzoeker, A-KO, Maastricht University).

During this double master program, he actively participated in the planning group of the scientific program and started publishing scientific papers. During the clinical internships, he started with the preparations for his $\mathrm{PhD}$ thesis. After graduating with a master thesis on the 'results of surgery for chronic groin pain after inguinal hernia repair, he started as a resident on the department of general surgery and the emergency department of Máxima Medical Center (MMC) in Eindhoven/Veldhoven. His scientific interest in postherniorrhaphy pain was combined with his work on the outpatient department of the centre of expertise for abdominal wall and groin pain (SolviMáx). Moreover, he participated in the board of MMC's 'PhD candidates club', was a reviewer for the Clinical Journal of Pain and co-authored a chapter in the book 'The Art of Hernia - A Step by Step Guide' (see List of Publications).

In June 2017, his scientific work was awarded a Best Clinical Research Award of the European Society for Surgical Research. From July 2017 on, Willem started his training as a general surgeon in the Maastricht University Medical Center under supervison of Prof. Dr. Laurents Stassen. He currently lives together with his girlfriend Leonie Benneker in Uden. 
Willem Antonius Richardus Zwaans werd geboren op 25 februari 1988 te Schaijk (Noord-Brabant). Hij behaalde zijn Athenaeum diploma (Mondriaan College Oss) in 2006 en werkte nadien gedurende één jaar als fulltime kok waarnaast hij Italiaans studeerde.

In 2007 startte hij met zijn studie 'Biomedische Wetenschappen' aan de Universiteit van Amsterdam en behaalde zijn bachelordiploma drie jaar later met zijn bachelorscriptie over 'neurally adjusted ventilatory assist (NAVA) versus pressure support ventilation in kritiek zieke patiënten (Academisch Medisch Centrum, Amsterdam). In datzelfde jaar werd hij geselecteerd voor de masteropleiding Arts-Klinisch Onderzoeker (A-KO, Maastricht University).

Tijdens deze combinatiemaster, nam hij deel aan de planningsgroep voor invulling van het wetenschappelijke programma en publiceerde Willem zijn eerste wetenschappelijke artikel. Tijdens de co-schappen begon hij met de voorbereidingen voor zijn promotietraject. Met zijn masterscriptie over de resultaten van chirurgie voor chronische liespijn na liesbreukherstel behaalde hij zijn diploma. Hij startte daarna als arts-assistent op de afdeling heelkunde en de spoedeisende hulp van Máxima Medisch Centrum (MMC) in Eindhoven/Veldhoven. Zijn wetenschappelijke interesse in liespijn na liesbreukherstel werd gecombineerd met werkzaamheden bij het expertisecentrum voor buikwand- en liespijn (SolviMáx). Daarnaast participeerde hij in de Wetenschapscommissie, was voorzitter van MMC's 'Promovendiclub', was reviewer voor 'the Clinical Journal of Pain' en co-auteur van een hoofdstuk over de etiologie van pijn in het boek 'The Art of Hernia - A Step by Step Guide' (zie List of Publications).

In juni 2017 ontving Willem de 'Best Clinical Research Award' voor zijn werk van 'the European Society for Surgical Research'. In juli 2017 start hij met de opleiding tot algemeen chirurg in het Maastricht Universitair Medisch Centrum (opleider Prof. Dr. Laurents Stassen). Hij woont momenteel samen met zijn vriendin Leonie Benneker in Uden. 
